\title{
Evaluation of the ATP Bioluminescence Assay as a Real-Time Monitoring Tool for Surface Water Monitoring and Disinfection Performance
}

\author{
A thesis submitted to \\ the Faculty of Graduate Studies and Research \\ in Partial Fulfillment of the requirements for the degree \\ Masters of Applied Science in \\ Environmental Engineering
}

by

Natalie Linklater

\begin{abstract}
Department of Civil and Environmental Engineering
Carleton University

Ottawa-Carleton Institute of Civil and Environmental Engineering
\end{abstract}

September 2010

(C)2010 Natalie Linklater 
Library and Archives

Canada

Published Heritage

Branch

395 Wellington Street

Ottawa ON K1A ON4

Canada
Bibliotheque et

Archives Canada

Direction du

Patrimoine de l'édition

395 , rue Wellington

Ottawa ON K1A ON4

Canada
Your file Votre référence

ISBN: 978-0-494-71515-4

Our file Notre référence

ISBN: $978-0-494-71515-4$
NOTICE:

The author has granted a nonexclusive license allowing Library and Archives Canada to reproduce, publish, archive, preserve, conserve, communicate to the public by telecommunication or on the Internet, loan, distribute and sell theses worldwide, for commercial or noncommercial purposes, in microform, paper, electronic and/or any other formats.

The author retains copyright ownership and moral rights in this thesis. Neither the thesis nor substantial extracts from it may be printed or otherwise reproduced without the author's permission.
AVIS:

L'auteur a accordé une licence non exclusive permettant à la Bibliothèque et Archives Canada de reproduire, publier, archiver, sauvegarder, conserver, transmettre au public par télécommunication ou par l'Internet, prêter, distribuer et vendre des thèses partout dans le monde, à des fins commerciales ou autres, sur support microforme, papier, électronique et/ou autres formats.

L'auteur conserve la propriété du droit d'auteur et des droits moraux qui protège cette thèse. $\mathrm{Ni}$ la thèse ni des extraits substantiels de celle-ci ne doivent être imprimés ou autrement reproduits sans son autorisation.
In compliance with the Canadian Privacy Act some supporting forms may have been removed from this thesis.

While these forms may be included in the document page count, their removal does not represent any loss of content from the thesis.
Conformément à la loi canadienne sur la protection de la vie privée, quelques formulaires secondaires ont été enlevés de cette thèse.

Bien que ces formulaires aient inclus dans la pagination, il n'y aura aucun contenu manquant.

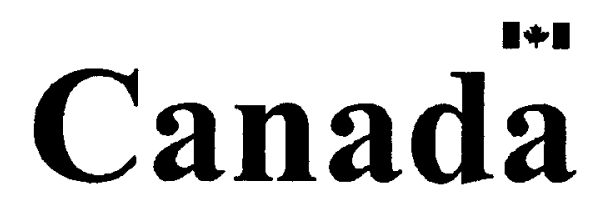




\section{AbSTRACT}

This paper reviewed current and emerging real-time monitoring technologies and evaluated the ATP bioluminescence assay for surface water monitoring and as a wastewater disinfection performance tool. The ATP assay measures light produced from the reaction between ATP and luciferin in presence of luciferase to quantify viable microbial cells.

Initial sensitivity limits of $10^{5} \mathrm{CFU} / \mathrm{mL}$ were improved to $10^{2} \mathrm{CFU} / \mathrm{mL}$ by increasing sample volumes. The assay was found to be suitable for use with surface water. Increasing turbidity from $2.56 \pm 0.1 \mathrm{NTU}$ to $32.43 \pm 0.55 \mathrm{NTU}$ did not interfere with results.

Parallel membrane filtrations and ATP assays of simulated wastewater before and after chlorination and UV irradiation with low pressure lamps were performed. The assay was not able to detect UV-induced cell damage but was able to detect chlorine-induced cell damage. With further improvements the ATP assay can potentially be used for surface water and wastewater disinfection monitoring. 


\section{ACKNOWLEDGEMENTS}

The completion of this study would not have been possible without the guidance of my supervisor Dr. Banu Ormeci. I thank you for your timely and insightful thoughts on all my work. It has truly been a pleasure working with you. To the members of my defence committee, I thank you for your comments and your review of this report. A special thanks to Dr. Marie-Josée Tudoret Chow who has always been happy and ready to help fulfill my requests.

To the members of my research group, I am grateful for your encouragement and inspiration. In particular, I am indebted to Kerim for "showing me the ropes" and to Audrey for her friendship and her careful review of this document. I would also like to extend my appreciation to all students, faculty, and staff in the department of Civil and Environmental Engineering. You have all made the transition into graduate studies most enjoyable.

To my family and friends, thank you for your unconditional love and support. Especially to Chris Fleming; I thank you for your patience and understanding. 


\section{Table of Contents}

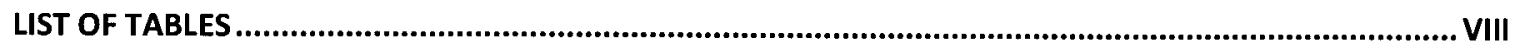

LIST OF FIGURES

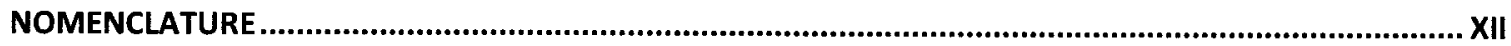

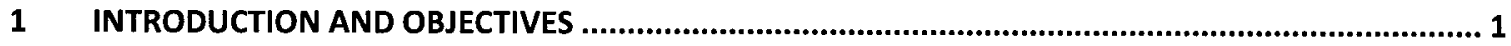

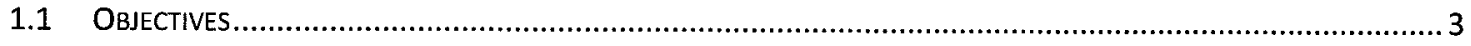

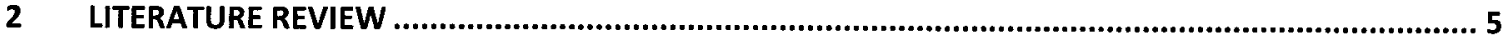

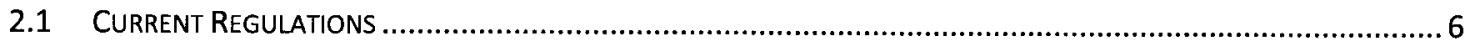

2.1.1 Guidelines for Canadian Drinking Water Quality.............................................................. 6

2.1.2 Ontario Drinking-water Quality Standards, Objectives and Guidelines................................ 7

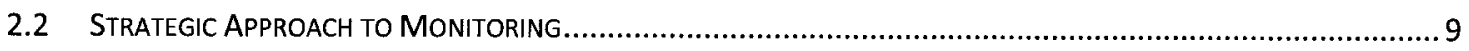

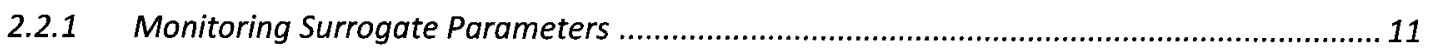

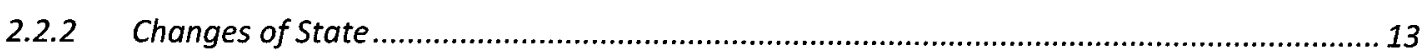

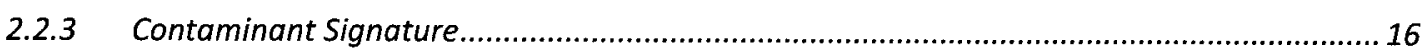

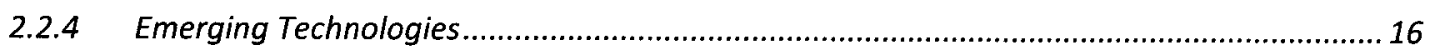

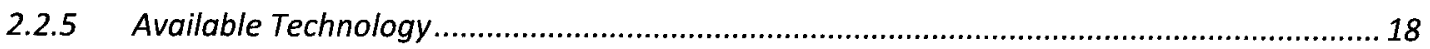

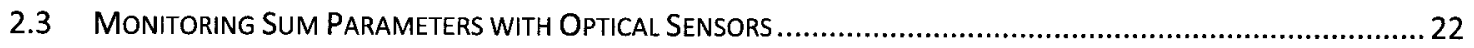

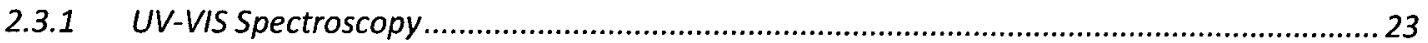

2.3.2 High Performance Size-Exclusion Chromatography (HPSEC) and UV Absorbance (UVa) ...... 24

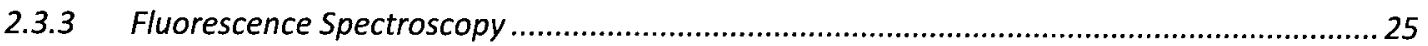

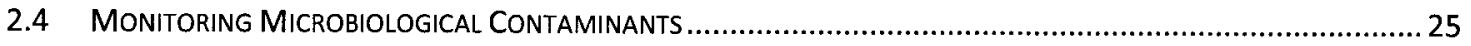

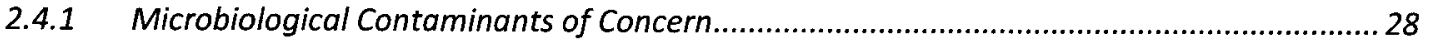

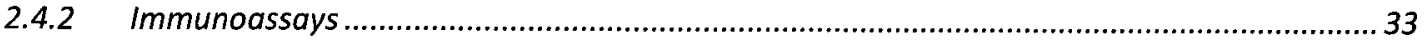

2.4.3 Conventional and Real-Time Quantitative Polymerase Chain Reaction ...............................37

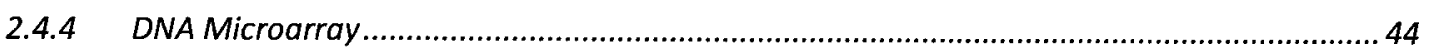

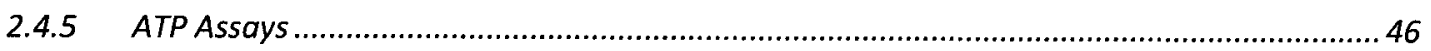

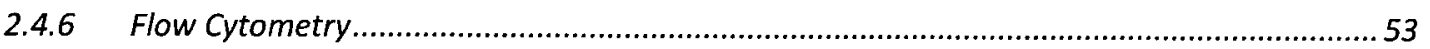

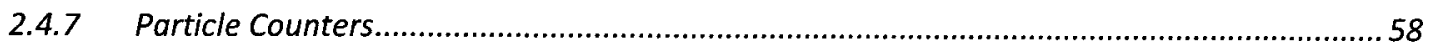

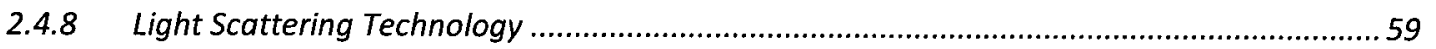

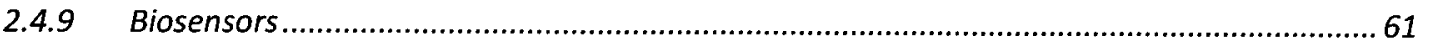

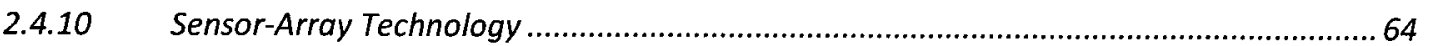




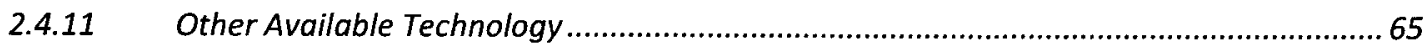

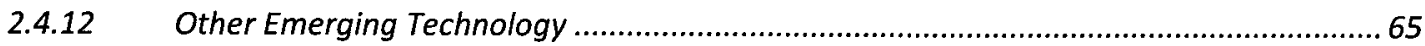

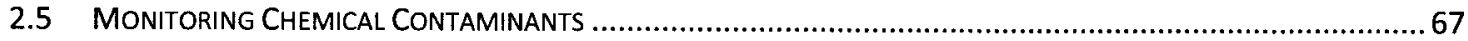

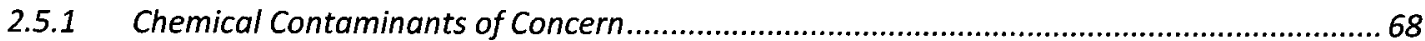

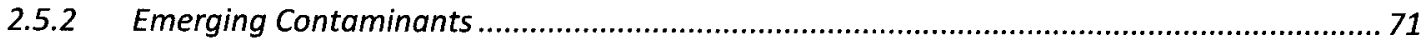

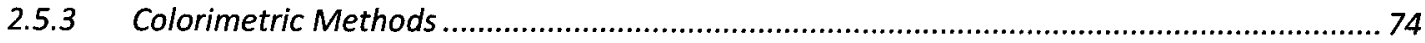

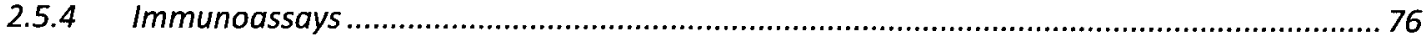

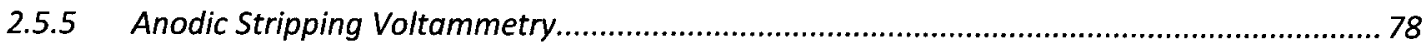

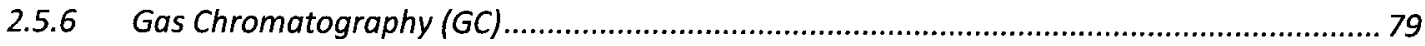

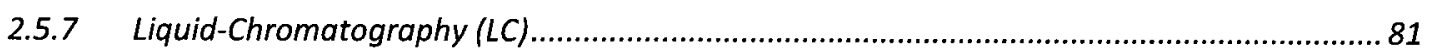

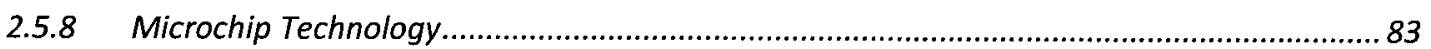

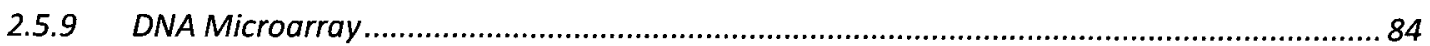

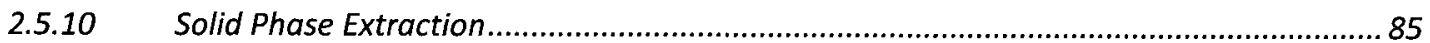

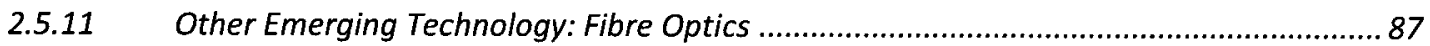

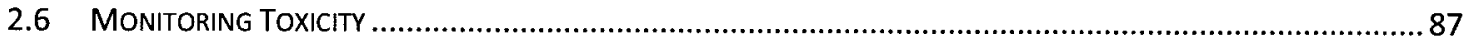

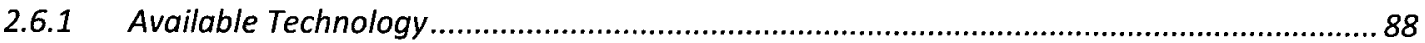

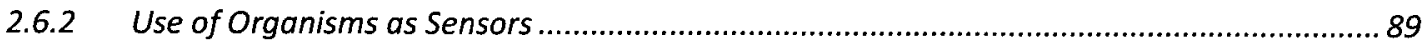

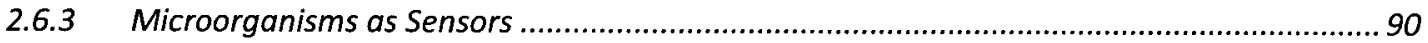

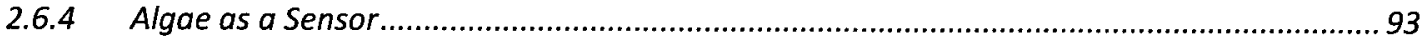

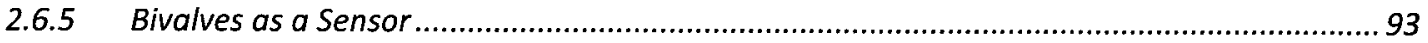

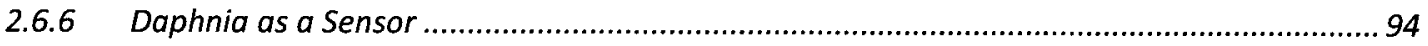

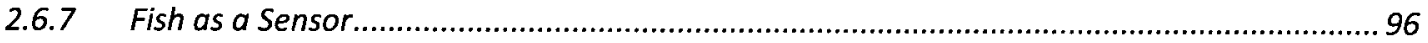

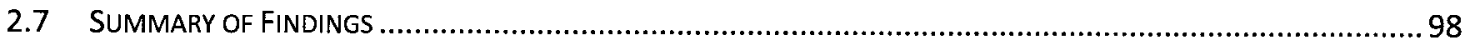

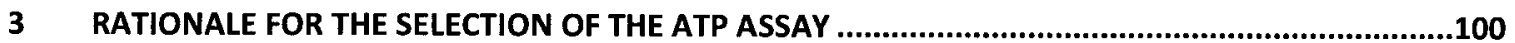

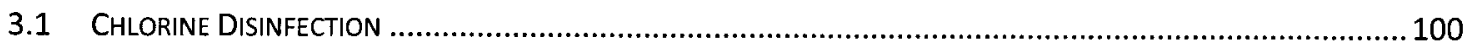

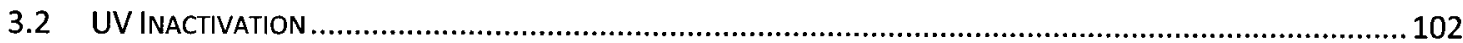

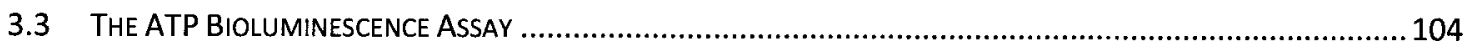

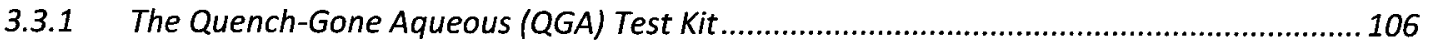

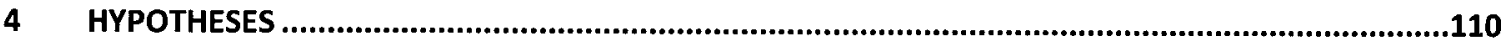




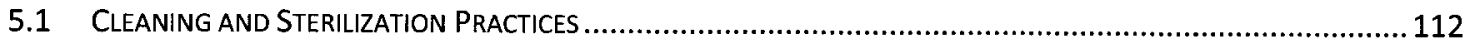

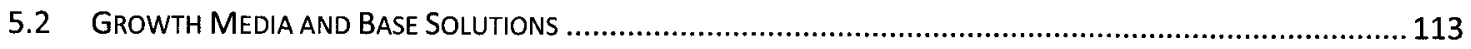

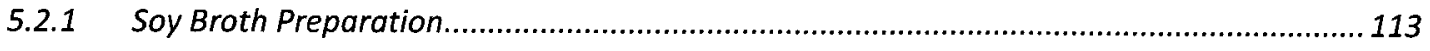

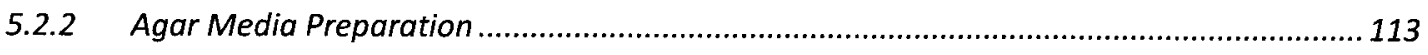

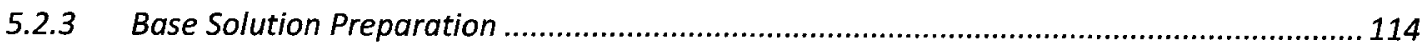

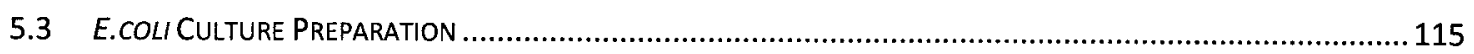

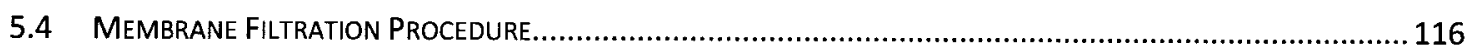

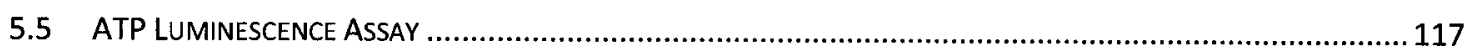

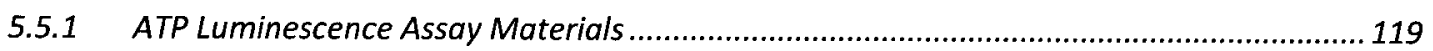

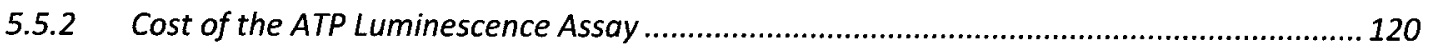

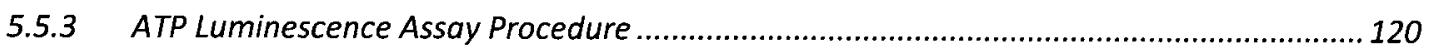

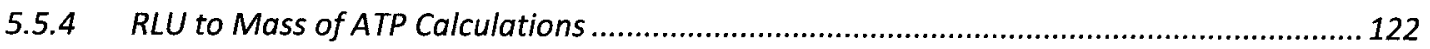

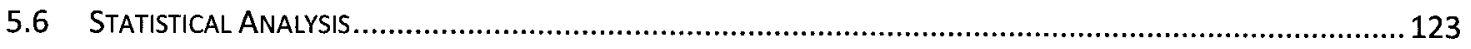

5.6.1 Calculating the sample mean and the sample variance .......................................... 123

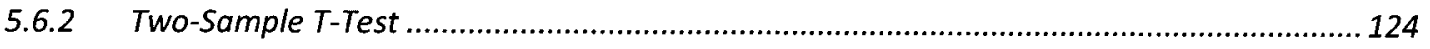

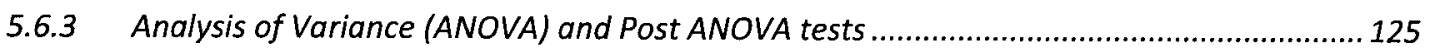

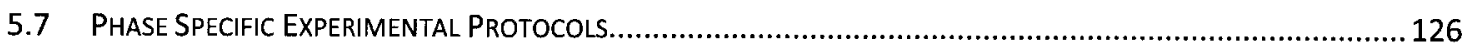

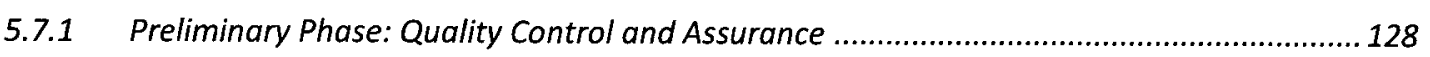

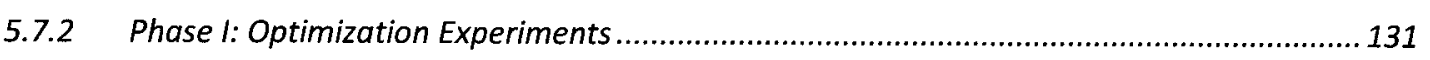

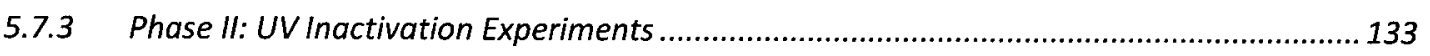

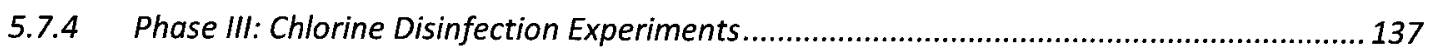

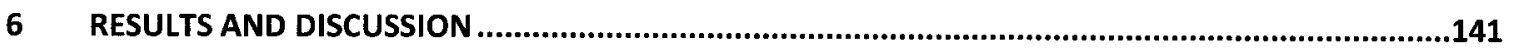

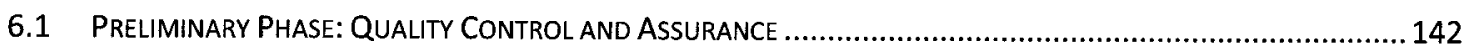

6.1.1 Light Leakage and Background Light Produced from Reagents .................................... 142

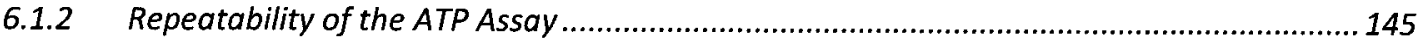

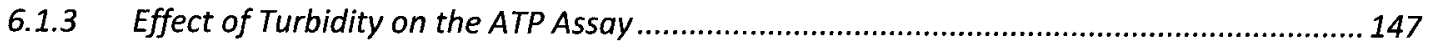

6.1.4 Calibration Curve with 0.1\% Peptone ...................................................................... 149

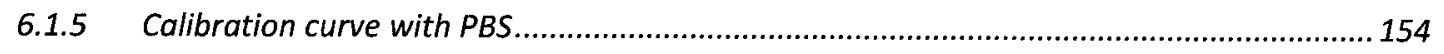

6.1.6 Calibration Curve For High Bacteria Concentrations .....................................................157

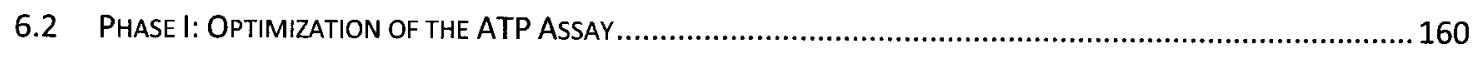

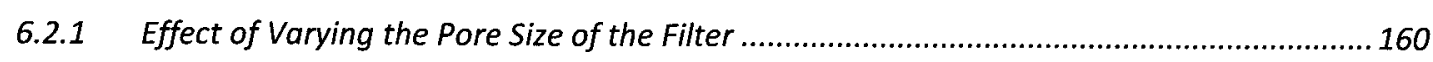

6.2.2 Effect of Varying the amount of Lysing Solution............................................................162

6.2.3 Calibration Curve for Low Bacteria Concentrations with XXL Luminase ..............................164 
6.2.4 Calibration Curve for High Bacteria Concentrations with XXL Luminase..........................171

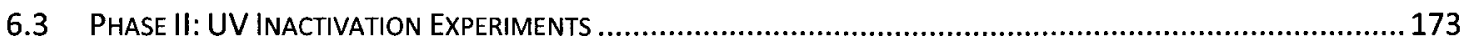

6.3.1 UV Dose Response Curves.............................................................................. 174

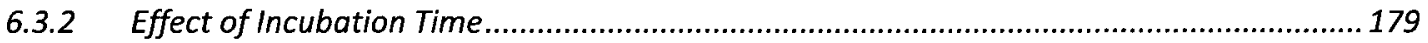

6.3.3 Effect of Incubation at Room Temperature ............................................................. 185

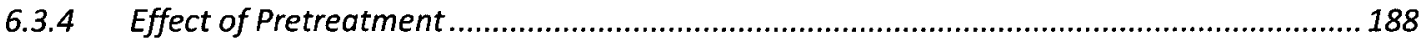

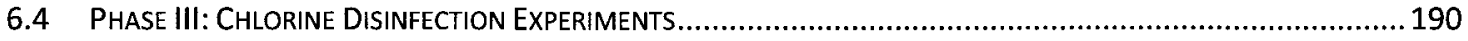

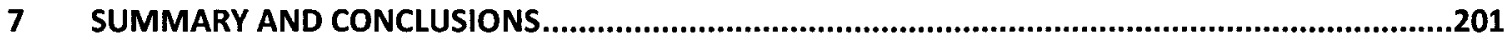

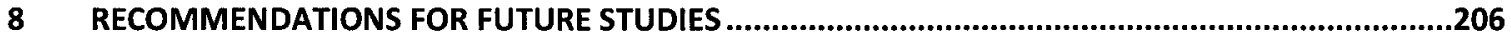

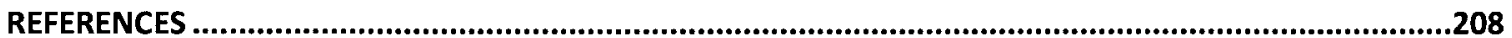

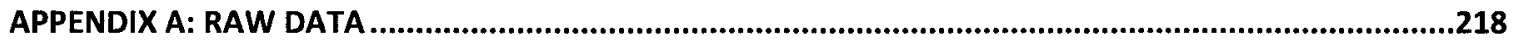

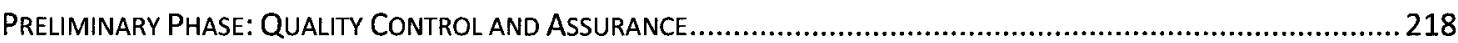

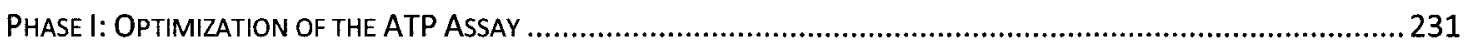

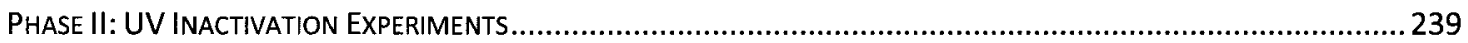

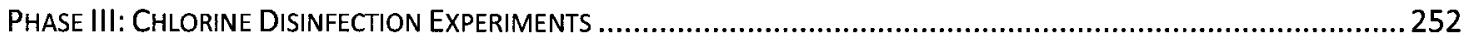




\section{LIST OF TABLES}

Table 2.1: Technology used in Online Sensors to Monitor for Physical and Chemical Parameter

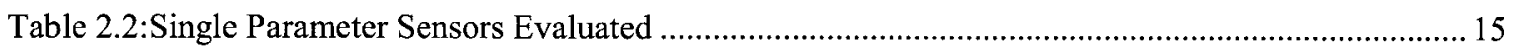

Table 2.3: Multi-Parameter Sensors Evaluated (Hall et al. 2007) ........................................................... 15

Table 2.4: Summary of Microbiological Contaminants of Concern.............................................................32

Table 2.5: Contaminants to be detected by Bio Threat Alert $\mathbb{B}($ EPA 2005) ................................................. 35

Table 2.6: Summary of US EPA ETV Results .....................................................................................95

Table 5.1: Experimental tasks completed for each of the four experimental phases .................................. 127

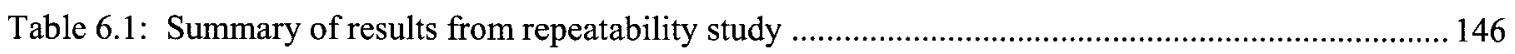

Table 6.2: Average amount of ATP measured using the ATP luminescence assay comparing $0.7 \mu \mathrm{m}$ and

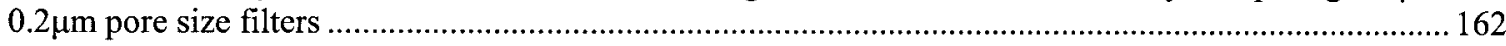

Table 6.3: Results from the post-ANOVA Dunnett test comparing individual data points with the control

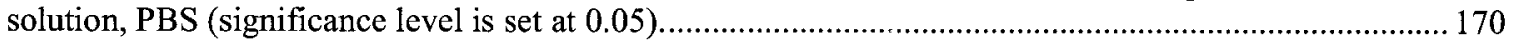

Table 6.4: Predicted and actual amounts of ATP using regular strength Luminase and XXL Luminase .... 172

Table 6.5: Results of the Dunnett's test using the ATP assay comparing sample points to that of the original

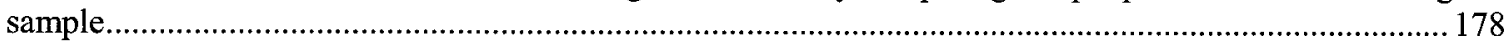

Table 6.6: Summary of the results of parallel membrane filtration and ATP assays of samples after the application of a pretreatment method. 189 


\section{LiST OF Figures}

Figure 3.1: Illustration of effect of UV light on the structure of DNA. Before, each purine is paired with a prymidine across the double helix structure. After, formation of prymidine dimmers within a helix. Source (Allen 2001).

Figure 3.2: Change of the amount of ATP of a surface water sample with time subsequent to UV irradiation with low and medium pressure UV lamps. Source: (LuminUltra Technologies) ......................................... 108

Figure 5.1: UV Collimated Beam Set-Up (source: Bolton and Linden 2003)

Figure 6.1: Results of the repeatability test of three bacteria concentrations $\left(10^{5}, 10^{3}\right.$ and $\left.10^{2} \mathrm{CFU} / \mathrm{mL}\right)$ where the average of five replicates is shown on a log-log scale. 146

Figure 6.2: Amount of ATP measured from a sample of water from the Rideau River with increasing turbidity. 148

Figure 6.3: Results of the membrane filtration showing the logarithm bacteria concentration of the logarithm of four dilutions of a sample with initial bacteria concentration of $1.1 \times 10^{5} \pm 1 \times 10^{4} \mathrm{CFU} / \mathrm{mL}$ (as measured by membrane filtration) with $0.1 \%$ peptone water. 152

Figure 6.4: Results of the ATP assay with Luminase showing the amount of ATP of the logarithm of five dilutions of a sample with initial bacteria concentration of $1.1 \times 10^{5} \pm 1 \times 10^{4} \mathrm{CFU} / \mathrm{mL}$ (as measured by membrane filtration) with $0.1 \%$ peptone water and $10.0 \mathrm{~mL}$ sample volumes.

Figure 6.5: Results of the ATP assay with Luminase showing the logarithm of the amount of ATP of the logarithm of five dilutions of a sample with initial bacteria concentration of $1.1 \times 10^{5} \pm 1 \times 10^{4} \mathrm{CFU} / \mathrm{mL}$ (as measured by membrane filtration) with $0.1 \%$ peptone water and $10.0 \mathrm{~mL}$ sample volumes. 153

Figure 6.6: Calibration curve between membrane filtration and ATP assay with Luminase using $10.0 \mathrm{~mL}$ samples and $0.1 \%$ peptone.

Figure 6.7: Results of the membrane filtration showing the logarithm bacteria concentration of the logarithm of four serial dilutions of a sample with initial bacteria concentration of $1.2 \times 10^{5} \pm 3 \times 10^{4} \mathrm{CFU} / \mathrm{mL}$ (as measured by membrane filtration) with PBS. 156

Figure 6.8: Results of the ATP assay with Luminase showing the logarithm of the amount of ATP of the logarithm of four dilutions of a sample with initial bacteria concentration of $1.2 \times 10^{5} \pm 3 \times 10^{4} \mathrm{CFU} / \mathrm{mL}$ (as measured by membrane filtration) with PBS and $10.0 \mathrm{~mL}$ sample volumes. 156

Figure 6.9: Calibration curve between membrane filtration and ATP assay using $10.0 \mathrm{~mL}$ samples and PBS.

Figure 6.10: Calibration curve between membrane filtration and ATP assay using solutions with high bacteria concentrations $\left(10^{5}\right.$ to $\left.10^{9} \mathrm{CFU} / \mathrm{mL}\right)$. 159

Figure 6.11: Varying the amount of lysing solution and effects of time 163

Figure 6.12: Results of the membrane filtration showing the logarithm bacteria concentration of the logarithm of three dilutions of a sample with initial bacteria concentration of $9.5 \times 10^{4} \pm 8 \times 10^{3} \mathrm{CFU} / \mathrm{mL}$ (as measured by membrane filtration) with PBS and low bacteria concentrations.

Figure 6.13: Calibration curve between membrane filtration and ATP assay using solutions with low bacteria concentrations $\left(10^{5}\right.$ to $\left.10^{-2} \mathrm{CFU} / \mathrm{mL}\right)$ and comparing regular strength Luminase with $\mathrm{XXL}$ Luminase. 
Figure 6.14: Results of the membrane filtration showing the logarithm bacteria concentration of the logarithm of six dilutions of a sample with initial bacteria concentration of $1.7 \times 10^{3} \pm 2 \times 10^{2} \mathrm{CFU} / \mathrm{mL}$ with PBS and low bacteria concentrations.

Figure 6.15: Calibration curve between membrane filtration and ATP assay using solutions with low bacteria concentrations $\left(1.7 \times 10^{3} \pm 2 \times 10^{2} \mathrm{CFU} / \mathrm{mL}\right)$ and XXL Luminase. 170

Figure 6.16: Calibration curve between membrane filtration and ATP assay using solutions with high bacteria concentrations $\left(10^{5}\right.$ to $\left.10^{9} \mathrm{CFU} / \mathrm{mL}\right)$ and comparing regular strength Luminase with $\mathrm{XXL}$ Luminase. 173

Figure 6.17: UV dose-response of an initial solution of $1.3 \times 10^{5} \pm 3 \times 10^{4} \mathrm{CFU} / \mathrm{mL}$ showing the $\log$ of the amount of bacteria as measured by membrane filtration and $0.1 \%$ peptone water 175

Figure 6.18: UV dose-response of an initial solution of $1.3 \times 10^{5} \pm 3 \times 10^{4} \mathrm{CFU} / \mathrm{mL}$ showing the amount of ATP as measured by ATP assay with $5.0 \mathrm{~mL}$ samples and $0.1 \%$ peptone water and Luminase. 176

Figure 6.19: UV dose-response of an initial solution of $1.5 \times 10^{5} \pm 2 \times 10^{4} \mathrm{CFU} / \mathrm{mL}$ showing the $\log$ of the amount of bacteria as measured by membrane filtration and PBS 177

Figure 6.20: UV dose-response of an initial solution of $1.3 \times 10^{5} \pm 3 \times 10^{4} \mathrm{CFU} / \mathrm{mL}$ showing the amount of ATP as measured by the ATP assay with PBS and XXL Luminase. 178

Figure 6.21: Results of 6-hour incubation at three different temperatures $\left(4^{\circ} \mathrm{C}, 21^{\circ} \mathrm{C}\right.$ and $\left.35^{\circ} \mathrm{C}\right)$ of an initial sample with a bacteria concentration of $2.2 \times 10^{5} \mathrm{CFU} / \mathrm{mL}$ exposed to a UV dose of $100 \mathrm{~mJ} / \mathrm{cm}^{2}$. 180

Figure 6.22: Results of the membrane filtration of a sample with initial bacterial concentration of $1.69 \times 10^{6}$ $\pm 2.0 \times 10^{5} \mathrm{CFU} / \mathrm{mL}$ exposed to no UV irradiation and incubated at $4^{\circ} \mathrm{C}$ for up to 144 hours ( 6 days). ....... 182

Figure 6.23: Results of the membrane filtration of a sample with initial bacterial concentration of $1.69 \times 10^{6}$ $\pm 2.0 \times 10^{5} \mathrm{CFU} / \mathrm{mL}$ exposed to UV radiation of a dose of $40 \mathrm{~mJ} / \mathrm{cm}^{2}$ and incubated at $4{ }^{\circ} \mathrm{C}$ for up to 144 hours (or 6 days).

Figure 6.24: Results of the membrane filtration of a sample with initial bacterial concentration of $1.69 \times 10^{6}$ $\pm 2.0 \times 10^{5} \mathrm{CFU} / \mathrm{mL}$ exposed to UV radiation of a dose of $60 \mathrm{~mJ} / \mathrm{cm}^{2}$ and incubated at $4^{\circ} \mathrm{C}$ for up to 144 hours (or 6 days)...

Figure 6.25: Results of the ATP assay of a sample with initial bacterial concentration of $1.69 \times 10^{6} \pm$ $2.0 \times 10^{5} \mathrm{CFU} / \mathrm{mL}$, as measured by membrane filtration, exposed to UV radiation of a dose of $0 \mathrm{~mJ} / \mathrm{cm}^{2}$ and incubated at $4^{\circ} \mathrm{C}$ for up to 144 hours ( 6 days). 184

Figure 6.26: Results of the ATP assay of a sample with initial bacterial concentration of $1.69 \times 106 \pm$ $2.0 \times 105 \mathrm{CFU} / \mathrm{mL}$, as measured by membrane filtration, exposed to UV radiation of a dose of $40 \mathrm{~mJ} / \mathrm{cm} 2$ and incubated at $4^{\circ} \mathrm{C}$ for up to 144 hours ( 6 days). 184

Figure 6.27: Results of the ATP assay of a sample with initial bacterial concentration of $1.69 \times 10^{6} \pm$ $2.0 \times 10^{5} \mathrm{CFU} / \mathrm{mL}$, as measured by membrane filtration, exposed to UV radiation of a dose of $60 \mathrm{~mJ} / \mathrm{cm}^{2}$ and incubated at $4^{\circ} \mathrm{C}$ for up to 144 hours (6 days). 185

Figure 6.28: Results of the membrane filtration of a sample with initial bacterial concentration of $1.9 \times 10^{5} \pm$ $3 \times 10^{4} \mathrm{CFU} / \mathrm{mL}$ exposed to UV radiation of a dose of $40 \mathrm{~mJ} / \mathrm{cm}^{2}$ and incubated at room temperature for 8 hours. 187

Figure 6.29: Results of ATP assay of a sample with initial bacterial concentration of $1.9 \times 10^{5} \pm$ $3 \times 10^{4} \mathrm{CFU} / \mathrm{mL}$ exposed to UV radiation of a dose of $40 \mathrm{~mJ} / \mathrm{cm}^{2}$ and incubated at room temperature for 8 hours.

Figure 6.30: Results of membrane filtration of a sample with initial bacteria concentration of $1.4 \times 10^{5} \pm 2 \times 10^{4} \mathrm{CFU} / \mathrm{mL}$ and exposed to an initial chlorine concentration of $0.72 \mathrm{mg} / \mathrm{L} \mathrm{Cl} \mathrm{Cl}_{2}$ for up to 45 minutes. 192

Figure 6.31: Results of membrane filtration of a sample with initial bacteria concentration of $1.3 \times 10^{5} \pm 5 \times 10^{4} \mathrm{CFU} / \mathrm{mL}$ and exposed to an initial chlorine concentration of $5.10 \mathrm{mg} / \mathrm{L} \mathrm{Cl}_{2}$ for up to 45 minutes. 
Figure 6.32: Results of membrane filtration of a sample with initial bacteria concentration of $3.0 \times 10^{5} \pm 3 \times 10^{4} \mathrm{CFU} / \mathrm{mL}$ and exposed to an initial chlorine concentration of $11.00 \mathrm{mg} / \mathrm{L} \mathrm{Cl} \mathrm{Cl}_{2}$ for up to 45 minutes.

Figure 6.33: Results of ATP assay of a sample with initial bacteria concentration of $1.4 \times 10^{5} \pm 2 \times 10^{4} \mathrm{CFU} / \mathrm{mL}$ and exposed to an initial chlorine concentration of $0.72 \mathrm{mg} / \mathrm{L} \mathrm{Cl}_{2}$ for up to 45 minutes. 194

Figure 6.34: Results of ATP assay of a sample with initial bacteria concentration of $1.3 \times 10^{5} \pm 5 \times 10^{4} \mathrm{CFU} / \mathrm{mL}$ and exposed to an initial chlorine concentration of $5.10 \mathrm{mg} / \mathrm{L} \mathrm{Cl}_{2}$ for up to 45 minutes. 194

Figure 6.35: Results of ATP assay of a sample with initial bacteria concentration of $3.0 \times 10^{5} \pm 3 \times 10^{4}$ $\mathrm{CFU} / \mathrm{mL}$ and exposed to an initial chlorine concentration of $11.00 \mathrm{mg} / \mathrm{L} \mathrm{Cl}_{2}$ for up to 45 minutes. 195

Figure 6.36: Results of membrane filtration of a sample with initial bacteria concentration of $2.0 \times 10^{5} \pm$ $5 \times 10^{4} \mathrm{CFU} / \mathrm{mL}$ and exposed to an initial chlorine concentration of $0.94 \mathrm{mg} / \mathrm{L} \mathrm{Cl}_{2}$ for 15 minutes. 197

Figure 6.37: Results of membrane filtration of a sample with initial bacteria concentration of $1.6 \times 10^{5} \pm$ $2 \times 10^{4} \mathrm{CFU} / \mathrm{mL}$ and exposed to an initial chlorine concentration of $0.56 \mathrm{mg} / \mathrm{L} \mathrm{Cl}_{2}$ for 10 minutes. 198

Figure 6.38: Results of membrane filtration of a sample with initial bacteria concentration of $1.5 \times 10^{5} \pm$ $2 \times 10^{4} \mathrm{CFU} / \mathrm{mL}$ and exposed to an initial chlorine concentration of $0.38 \mathrm{mg} / \mathrm{L} \mathrm{Cl}_{2}$ for 15 minutes. 198

Figure 6.39: Results of the ATP assay using XXL Luminase and $100 \mathrm{~mL}$ volumes of a sample with initial bacteria concentration of $2.0 \times 10^{5} \pm 5 \times 10^{4} \mathrm{CFU} / \mathrm{mL}$ and exposed to an initial chlorine concentration of 0.94 $\mathrm{mg} / \mathrm{L} \mathrm{Cl}{ }_{2}$ for 15 minutes.

Figure 6.40: Results of the ATP assay using XXL Luminase and $100 \mathrm{~mL}$ volumes of a sample with initial bacteria concentration of $1.6 \times 10^{5} \pm 2 \times 10^{4} \mathrm{CFU} / \mathrm{mL}$ and exposed to an initial chlorine concentration of $0.56 \mathrm{mg} / \mathrm{L} \mathrm{Cl}_{2}$ for 15 minutes. 199

Figure 6.41: Results of the ATP assay using XXL Luminase and $100 \mathrm{~mL}$ volumes of $1.5 \times 10^{5} \pm 2 \times 10^{4}$ $\mathrm{CFU} / \mathrm{mL}$ and exposed to an initial chlorine concentration of $0.38 \mathrm{mg} / \mathrm{L} \mathrm{Cl}{ }_{2}$ for 15 minutes. 200 


\section{NOMENCLATURE}

$\begin{array}{ll}\text { ANOVA } & \text { Analysis of variance } \\ \text { ASV } & \text { Anodic stripping voltametry } \\ \text { As } & \text { Arsenic } \\ \text { AsH3 } & \text { Arsine Gas } \\ \text { ATP } & \text { Adenosine triphosphate } \\ \text { BOD } & \text { Biochemical oxygen demand } \\ \text { BOD5 } & \text { Five-day biochemical oxygen demand } \\ \text { C } & \text { Carbon } \\ \text { CARD } & \text { Catalyzed Reported Disposition } \\ \text { CDC } & \text { Center for Disease Control and Prevention } \\ \text { CFU } & \text { Colony forming units } \\ \text { CHO } & \text { Chinese Hamster Ovary } \\ \text { CO2 } & \text { Carbon dioxide } \\ \text { COD } & \text { Chemical oxygen demand } \\ \text { CWS } & \text { Contamination warning system } \\ \text { DFO } & \text { Department of Fisheries and Oceans } \\ \text { DNA } & \text { Deoxyribonucleic acid } \\ \text { DO } & \text { Dissolved oxygen } \\ \text { DOC } & \text { Dissolved organic carbon } \\ \text { DOM } & \text { Dissolved organic matter } \\ \text { ECD } & \text { Electron capture detector } \\ \text { E.coli } & \text { Escherichia coli } \\ \text { EDC } & \text { Endocrine disrupting chemicals } \\ \text { ELISA } & \text { Enzyme-linked immunosorbent assay } \\ \text { EPA } & \text { Environmental Protection Agency } \\ \text { EWS } & \text { Early Warning System } \\ & \end{array}$


FID Flame ionization detector

FISH Fluorescence in-situ hybridization

FITC Fluorescein isothiocyanate

g grams

GC Gas Chromatography

GCDWQ Guidelines for Canadian Drinking Water Quality

GHAA Gaseous hydride formation

$\mathrm{H} \quad$ Hydrogen

h Hours

HAV Herpes simplex virus

HILIC Hydrophilic interaction liquid chromatography

HPC Heterotrophic plate count

HPLC High-pressure liquid chromatography

HPLC- High-pressure liquid chromatography coupled with UV adsorption UVD detection

HPSEC High performance size-exclusion chromatography

HRP Horseradish peroxidase

HUS Haemolytic-uraemic syndrome

IAC Immuno affinity chromatography

ICP-AES Inductively coupled plasma atomic emission spectroscopy

ICP-MS Inductively coupled plasma mass spectroscopy

IMS Immunomagnetic separation

L Litres

LC Liquid Chromatography

LC-EMS- Liquid chromatography-electrospray ionization mass spectrometry
MS

LC-ESI- Liquid chromatography hyphenated with electro-spray ionization triple MS/MS quadrupole mass spectrometry

LC/TOF- Liquid chromatography columns and a time of flight mass MS spectroscopy

LOD Limit of detection

LOQ Limit of quantification

m Meter 


$\begin{array}{ll}\text { mg } & \text { Milligrams }\left(10^{-3} \text { g) }\right. \\ \text { mL } & \text { Millilitres }\left(10^{-3} \mathrm{~L}\right) \\ \text { Mac } & \text { Mycobacterium avium complex } \\ \text { MALS } & \text { Multi-angle light scattering } \\ \text { MIPs } & \text { Molecularly imprinted synthetic polymers } \\ \text { MOE } & \text { Ontario Ministry of the Environment } \\ \text { MS } & \text { Mass spectroscopy } \\ \text { MS/MS } & \text { Tandem mass spectrometric technique } \\ \text { nm } & \text { Nanometres }\left(10^{-9} \text { m }\right) \\ \text { NTU } & \text { Nephelometric turbidity units } \\ \text { O } & \text { Oxygen } \\ \text { OECD } & \text { Organisation for Economic Co-operation and Development } \\ \text { OFWDW } & \text { Office of Ground Water and Drinking Water } \\ \text { OPR } & \text { Oxidation-reduction potential } \\ \text { pg } & \text { Pico gram }\left(10^{-12} \text { g) }\right. \\ \text { PAH } & \text { Polycyclic aromatic hydrocarbons } \\ \text { PBS } & \text { Phosphate buffered solution } \\ \text { PBDE } & \text { Polybrominated diphenyl ether } \\ \text { PCR } & \text { Polymerase chain reaction } \\ \text { PEC } & \text { Photosynthetic enzymatic complex } \\ \text { PID } & \text { Photo ionization detector } \\ \text { PPCP } & \text { Pharmaceutical and personal care products } \\ \text { PPE } & \text { Polyphenylene } \\ \text { QGA } & \text { Quench Gone Aqueous } \\ \text { RAM } & \text { Restricted access material } \\ \text { R.A.P.I.D. } & \text { Ruggedized advanced pathogen identification device } \\ \text { RIANA } & \text { River Analyser } \\ \text { RT-PCR } & \text { Real-time polymerase chain reaction } \\ \text { RLU } & \text { Relative light uints } \\ \text { SAW } & \text { Surface acoustic wave } \\ \text { SCADA } & \text { Supervisory Control And Data Acquisition } \\ \text { SELDI } & \text { Surface-enhanced laser desorption ionization } \\ & \end{array}$




$\begin{array}{ll}\text { SIM } & \text { Surface acoustic wave } \\ \text { SPE } & \text { Solid phase extraction } \\ \text { TCD } & \text { Thermal conductivity detector } \\ \text { TIRF } & \text { Total internal reflection fluorescence } \\ \text { TOC } & \text { Total organic carbon } \\ \text { TSA } & \text { Tyramide signal amplification } \\ \text { TSS } & \text { Total suspended solids } \\ \text { UK } & \text { United Kingdom } \\ \text { US or USA } & \text { United States of America } \\ \text { UV } & \text { Ultraviolet } \\ \text { UVa } & \text { Ultraviolet absorbance } \\ \text { VOCs } & \text { Volatile organic compounds } \\ \text { WERF } & \text { Water Environment Research Federation } \\ \text { WHO } & \text { World Health Organization } \\ \mu m & \text { Micrometers (10 } 0^{-6} \text { m) } \\ { }^{\circ} \mathrm{C} & \text { Degree Celsius }\end{array}$




\section{CHAPTER 1}

\section{INTRODUCTION AND OBJECTIVES}

Effective monitoring of water supplies is critical for the protection of public health and environment. Traditionally, water quality evaluation has depended on time- and labourintensive on-site sample collection and transport to a laboratory. Analysis of samples typically takes several days and the data may be compromised due to sampling errors and extended holding times. Real-time monitoring, on the other hand, provides timely and continuous reliable data and a quick response time for authorities in case of emergencies. On-line and real-time monitoring of water supplies has become a priority in recent years due to the present vulnerability of the water supplies and distribution systems, and the continuing threat of intentional attacks, failing infrastructure and contamination from industrial sources. Real-time data can be used to assess changes to water quality and identify trends, determine the state of water quality and ecosystem health, identify emerging issues and contaminants, and achieve rapid screening of water for toxic substances and pathogens. Another important application is the monitoring and optimization of water and wastewater treatment processes and ensuring compliance with water quality standards. In spite of the need, presently there are limited options for the implementation of real-time monitoring of water quality and quantity. In addition, the capacity to develop and undertake real-time monitoring is poorly understood and not well connected. 
Real-time sensors are more limited compared to the laboratory methods, but their variety and capabilities are continuously improving. The majority of the current real-time monitoring applications are based on one or more of the following water quality parameters: turbidity, conductivity, temperature, dissolved oxygen, $\mathrm{pH}$, and chlorophylla. More recently systems that can measure ultraviolet absorbance at $254 \mathrm{~nm}$ (UVA) and total organic carbon (TOC) have also been used as an indirect measurement of dissolved organic matter. These parameters are relatively easy to measure and provide useful information on the daily, weekly, monthly and seasonal changes in water quality. Sudden and uncharacteristic changes in these parameters also serve as an early warning system against intentional contamination of water with toxic chemicals or biological agents. However, none of these parameters can identify a specific chemical or a biological agent, and they merely serve as a screening method. Efforts continue to employ advanced spectroscopy and molecular methods in in-situ detectors, but majority are still in the research phase and not ready for field applications. Other methods include using organisms such as fish, clams, mussels, daphnia, and algae as biomonitors, bioluminescence-based assays for toxicity testing, rapid quantification of viable bacteria using an adenosine triphosphate (ATP) assay, and employing immunomagnetic separation methods and flow cytometry.

This research consists of two main parts. The first part, which was initiated by a request from the Ontario Centres of Excellence (OCE), undertook a thorough literature review of the existing and emerging technologies for monitoring water and wastewater quality. Based on the findings of the literature review, physical, chemical and biological detection methods that can be turned into real-time or near real-time monitoring tools were 
identified. In addition, consideration was given to Ontarian and Canadian companies with products that can be developed into real-time monitoring systems. At the end of the first part, ATP Bioluminescence Assay emerged as a possible candidate for real-time monitoring of microbial content of water supplies as well as for disinfection monitoring at wastewater treatment plants. The second part of this study evaluated the performance of the ATP Bioluminescence Assay in accurately determining low and high levels of bacteria numbers in water samples under a wide range of conditions.

\subsection{ObJECTIVES}

This study had the following objectives.

1. Carry out a thorough literature review of the current state of knowledge in realtime monitoring of water supplies.

2. Identify existing and emerging detection methods that can be converted to realtime or near real-time monitoring technologies, and compare their advantages and limitations.

3. Identify possible research and development opportunities with Ontarian and Canadian companies.

4. Choose one of the most promising technologies based on the results of the literature review.

5. Experimentally determine the sensitivity and performance of the selected method for monitoring water supplies.

6. Experimentally determine the sensitivity and performance of the selected method for monitoring treatment processes 
To complete the objectives of this study a literature review of current technologies is presented in Chapter 2. Further, the rationale for the selection of the detection method (ATP Assay) for further testing is presented in Chapter 3. The materials and methods to complete the evaluation of the assay can be seen in Chapter 5 and the results and discussion of the study are presented in Chapter 6. Finally, a summary of the results and the conclusions of this study can be found in Chapter 7 and the recommendations for future studies are presented in Chapter 8 . 


\section{CHAPTER 2}

\section{Literature REVIEW}

This Chapter aims to review the current and emerging detection methods for physical, chemical and biological water parameter. From this, it is hoped that monitoring tools and strategies can be identified for the use in the real-time, or near real-time, water quality of our water supplies. With a particular attention being paid to Canadian companies, this review also lists products and technologies currently on the market that can be developed or integrated into real-time monitoring systems.

The chapter begins with a review of current regulations surrounding the monitoring requirements of Canada's water supply (Section 2.1). Modern water issues include intentional contamination where constant monitoring and a quick response are vital to the protection of public health. The development of a tried and strategic approach to monitoring is important to screen for contamination and allow time to mediate the problem. The use of on-line real-time sensors that are already in place is practical and economical solution (Section 2.2). Following, new optical sensors are describe, which includes the use of spectroscopy to measure total organic carbon (TOC), biochemical oxygen demand (BOD) and dissolved oxygen (DO) (Section 2.3). New biological contamination monitoring methods are faster and more specific than traditional methods. Methods such as, conventional and real-time PCR, DNA microarrays as well as the ATP assay are investigated (Section 2.4). For chemical contaminants, the current focus is to develop new detection and better concentration methods for emerging contaminants 
(Section 2.5). Finally, the use of biological organisms, microorganism, and single cells as a sentry alarm systems is investigated (Section 2.6).

\subsection{Current Regulations}

The quality of drinking water and wastewater effluents in Canada are the shared responsibility of the municipal, provincial and federal governments. Protection of public and environmental health and communication with the public is at the centre of all regulations. A public communication plan is required as part of the water monitoring regimes of water and wastewater treatment plants. This plan must include information about communicate possible risks to the public, making reports available and having opportunities for public consultations (Canadian Council Of Ministers of the Environment (CCME) and Health 2002).

\subsubsection{GUIDELINES FOR CANADIAN DRINKING WATER QUALITY}

The Guidelines for Canadian Drinking Water Quality (GCDWQ) aim to protect receiving bodies of water, aquatic life, sensitive agricultural crops and human health against toxic chemicals, harmful microorganisms and aesthetically displeasing compounds (Health Canada 2008).

A multi-barrier approach directs the monitoring requirements of the GCDWQ. A multiple barrier approach establishes barriers against all possible threats to the water system to assure that such threats are minimized or eliminated. It begins with identification of all possible threats that can arise from the source water to the distribution system and includes all naturally occurring and human made contaminants. Land use surveys in the drinking water catchment area are important to determine the potential contamination 
threats. Knowing all the vulnerabilities of a system allows for the appropriate selection of treatment and associated monitoring. (Health Canada 2008)

According the GCDWQ, water monitoring should occur:

- At the source to be able to modify treatment;

- During treatment to assess the efficacy of the treatment, and;

- In the distribution system to assure the safety of the water for public consumption (Health Canada 2008).

Water monitoring is also used to identify trends and make treatment changes as required. The monitoring frequency and other requirements vary depending on the contaminant (Health Canada 2008).

The Guidelines for Canadian Drinking Water Quality - Technical Documents (Health Canada 2006a) provides recommendations on the testing methods that should be employed for a long list of microbiological, chemical, physical and radiological parameters. For selected parameters, such as turbidity, these recommendations include the use of on-line instruments and regular sampling intervals (Health Canada 2006a).

\subsubsection{ONTARIO DRINKING-WATER QUALITY STANDARDS, OBJECTIVES AND GUIDELINES}

Ontario Drinking-Water Quality Standards Regulation (O.Reg 169/03) prescribes Ontario's drinking water standards. The standard aims to have drinking water that is free of disease-causing organisms and unsafe concentrations of chemical toxins and radiological substances. The regulation also limits parameters that can cause aesthetic 
issues (e.g. taste, odour and colour) or would have a negative effect on the distribution system (e.g. corrosion of pipes) (Ontario Ministry of the Environment (MOE) 2003).

Microorganisms are associated with many waterborne diseases and water should be free of viruses and protozoans. The regulation requires that there be no detectible Escherichia coli (E.coli) or total coliform in the water. The biosafety of water is indirectly monitored with the level of turbidity. After filtration, monitoring turbidity assures low levels of Giardia cysts and Cryptosporidium oocysts. The regulation also recommends limiting nutrients entering treatment and maintaining a clean distribution system to reduce microorganism growth (MOE, 2003).

The regulation includes a long list of chemical compounds and associated concentration limits. The regulation limits the concentration of heavy metals, inorganic and organic chemical compounds including pesticides. Radioactive material can cause cancer and hereditary genetic changes. It is difficult if not impossible to remove radioactive material during treatment and thus important that radioactivity be monitored to be below limits (MOE, 2003).

Drinking Water Systems Regulation (O.Reg 170/03) describes the number and frequency of microbiological and chemical sampling, testing and reporting requirements of a drinking water system. However, there are no real-time or online monitoring requirements for any contaminant (MOE, 2008). 


\subsection{Strategic APProACh TO Monitoring}

Modern water quality monitoring is tasked with detecting increasing amounts of contaminants, which may escape traditional water quality monitoring regimes. These include environmental and anthropogenic sources of pollution such as natural microbial outbreaks, agricultural runoff, chemical and biological warfare agents, radioactive contaminants and contaminants of emerging concern such as pharmaceutical and personal care products (PPCP). The response time to a given contamination depends on the quick detection and identification of the contaminant and having the ability of dispatching an appropriate response.

With limited funds and increasingly complex water monitoring issues, Canadian municipalities and water utilities are looking to operate water quality monitoring strategically. A Contamination Warning System (CWS) or Early Warning System (EWS) uses a tiered approach to monitor water quality. Such a system would monitor for general changes in water quality to screen for possible contaminants. Then, more specific testing would identify the type and extent of contamination. The aim of a CWS/EWS is to provide a surveillance system that can detect contamination, confirm the nature of the contaminant, predict its effects, communicate with the public and allow enough time for mitigation. Any comprehensive CWS/EWS should also include the use of consumer complaints, emergency response and community health-based data to protect the water supply and distribution system from contamination [Hall et al. 2007; United States Environmental Protection Agency (EPA), 2005]. 
When developing a CWS/EWS it is also important to consider (EPA 2005):

- Fate and transport of pathogens: Use of hydraulic models is useful to predict the where contamination will first occur. In addition, if the contaminant can be identified and is known to degrade, then, its impact can also be predicted.

- Sensor location and density: Sensors should be placed strategically to optimize their location and density. To do this, system characterization studies as well as vulnerability and threat analysis of the water source and distribution system are useful. A conventional water treatment train is able to remove some forms of contamination. However, there is no additional treatment once the treated water enters the distribution system. The distribution system is therefore more vulnerable to intentional contamination and sensor placement, and mitigations strategies need to be planned accordingly.

- Data management: Real-time systems are coupled with a large amount of data that has to be managed. A system must be developed to managing the continuous influx of data.

A strategic approach to water monitoring may also include the selection of specific contaminants to be tested and at what frequency. The amount of testing methods selected may depend on financial restriction as well as regulatory requirements. Land surveys and chemical uses in the area can help determine a list of most probable contaminants to look for. Prioritization should also be given to tests that are easy to perform (especially if able to be done on-site), that can detect a substance that presents a high risk to public health or 
that can give a wide description of the quality of the water (Gheewala et al. 2003). Increasingly, real-time testing methods are an important part of a water quality testing regime. In particular, they allow for the detection of highly toxic substances that may not yet be regulated.

\subsubsection{Monitoring SURRogate PARAMETERS}

Online sensors to monitor the physical and chemical characteristics of water are widely available and used by most modern water and wastewater treatment facilities for process control and regulatory compliance reporting. These sensors can measure parameters such as chlorine residuals, temperature, dissolved oxygen (DO), oxidation-reduction potential (ORP), $\mathrm{pH}$, conductivity, nitrogen compounds and ammonia compounds (see Table 2.1 for a summary of the technology used for different types of online sensors). Multiparameter sensors are able to measure a number of parameters with a single instrument and are typically composed of a number of single parameter sensors with a shared casing. Both single parameter and multi-parameter sensors are able to provide online data.

Managing water contamination may need a variety of steps before analytical confirmation of a contaminant can be determined. The use of surrogate parameters has been suggested as an initial step to warn that a new contaminant has been introduced in the water. Parameters already being monitored online, such as $\mathrm{pH}$, chlorine residuals and conductivity can change in the presence of a contaminant. If these changes can be identified, they can serve as a warning of contamination, trigger additional testing and be used as a tool for early identification and possible solutions to the problem.

Online sensors used in a contaminant warning system capacity is attractive to water utilities as these sensors are doubling their purpose and increasing their value (EPA 
2005). Online sensors are also able to provide real-time water quality characteristics, and contamination events can be detected allowing for a quicker response time.

Two strategies have been suggested to use single and multi-parameter sensors to screen for contaminants in water (EPA 2005). The first strategy would monitor changes in the state of the water to identify contamination. The second strategy attempts to establish contaminant signatures that would identify the specific contaminant in the water. The following sections discuss the two above-mentioned strategies.

Table 2.1: Technology used in Online Sensors to Monitor for Physical and Chemical Parameter

\begin{tabular}{|l|l|}
\hline \multirow{4}{*}{ Parameter } & Technology \\
\hline \multirow{4}{*}{ Chlorine } & Calorimetric and membrane electrode \\
\cline { 2 - 2 } & Ion-selective electrode $\left.\mathrm{Cl}^{-}\right)$ \\
\cline { 2 - 2 } & $\begin{array}{l}\text { Electro-chemical reduction of chlorine species at a micro- } \\
\text { electrode }\end{array}$ \\
\hline \multirow{3}{*}{ Demperature } & Thermistor \\
\hline \multirow{2}{*}{ Oxygen Reduction Potential } & Pembrane electrode \\
\cline { 2 - 2 } & Optical sensor \\
\cline { 2 - 2 } & Electrochemical voltametric \\
\hline \multirow{3}{*}{ Turbidity } & Glass bulb electrode \\
\cline { 2 - 2 } & Potentiometric measurement with reference electrode \\
\hline Conductivity & Mephelometric method \\
\hline Nitrogen Compounds & Optical sensor \\
\hline Ammonia Compounds & Conductivity cell method \\
\hline
\end{tabular}

Source: (EPA 2005) 


\subsubsection{CHANGES OF STATE}

The sum of the physical and chemical parameters measured by online sensors describes the state of water. The expected or typical state of water is described by baseline conditions determined for the specific water. A change of state is a significant deviation from baseline conditions of any one or a combination of the parameters monitored. Any change of state would suggest that a contaminant has been added to the water.

The US Environmental Protection Agency (EPA), in collaboration with private sector companies, is currently performing a variety of tests to determine the long-term performance of multi-parameter sensors, and is also establishing baseline conditions. One of these studies (Hall et al. 2007), performed at the WATERS research centre in Cincinnati Ohio, evaluated water quality monitors for the ability to detect changes due to chemical, physical and microbial contaminants. The study examined a small number of commercial sensors to determine what type of water quality parameters respond to the greatest amount of contaminants. The sensors studied included commercially available single and multi-parameter sensors that allowed for continuous, online monitoring of water supplies (for a list of single- and multi-parameter sensors evaluated during this study see Table 2.2 and Table 2.3 respectfully). The contaminants investigated included non-chlorinated secondary wastewater, potassium ferricyanide, malathion (pesticide), glyphosate (herbicide), arsenic trioxide, nicotine, aldicarb (pesticide), and E.coli K-12. Contaminants were injected into an uncontaminated pipe-loop where the sensor responses could be observed. To assure the accuracy of the results, a number of injections were performed for each contaminant. The results showed that each contaminant induced a response from at least one parameter. As predicted, total organic carbon (TOC) increased 
with the addition of carbon containing contaminants such as non-chlorinated wastewater, nicotine, E.coli, herbicides and pesticides. In addition, the specific conductance was responsive to the introduction of ionic compounds such as non-chlorinated wastewater and potassium ferricyanide. The ORP was responsive to oxidizing compounds or an increase in chlorine demand, which was the case with non-chlorinated wastewater, potassium ferricyanide, pesticides and the herbicide. The most responsive parameters were free chlorine, TOC, specific conductance and ORP. Although free chlorine was the only parameter that responded to all contaminants, its response to potassium ferricyanide is considered a false positive. Potassium ferricyanide is a strong oxidant able to oxidize the reagent of the chlorine sensor. No reaction between chlorine and potassium ferricyanide is expected to have occurred.

Chlorine reacts readily with many compounds and thus a decrease of free chlorine is a good indication of contamination. However, the single use of free chlorine as an indication of contamination is not recommended as some contaminates may not readily react with free chlorine or provide false positives. In addition, the use of free chlorine as an indicator also assumes that a chlorine residual exists in the water. While this is the case for most distribution systems, this is not the case for incoming water to treatment facilities (Hall et al. 2007).

The magnitude of the sensor response depended on the concentration of the contaminant injected. A sensor response to a contaminant may also depend on the specific baseline conditions of the water and thus the accuracy and efficiency of contaminant identification may be specific to the baseline conditions (Hall et al. 2007). 
Table 2.2:Single Parameter Sensors Evaluated

\begin{tabular}{|l|l|}
\hline \multicolumn{1}{|c|}{ Sensor Manufacture/Name } & Parameter(s) Measured \\
\hline ATI & Free Chlorine \\
\hline Hach Model A-15 Cl-17 & Free/Total Chlorine \\
\hline Hach 1720D & Turbidity \\
\hline GLI Model PHD & $\mathrm{pH}$ \\
\hline GLI Model 3422 & Specific Conductance \\
\hline Hach Asdtro TOC UV process Analyzer & Total Organic Carbon \\
\hline
\end{tabular}

Source: (Hall et al. 2007)

Table 2.3: Multi-Parameter Sensors Evaluated (Hall et al. 2007)

\begin{tabular}{|l|l|}
\hline \multicolumn{1}{|c|}{$\begin{array}{c}\text { Sensor } \\
\text { Manufacture/Name }\end{array}$} & \multicolumn{1}{c|}{ Parameters Measured } \\
\hline $\begin{array}{l}\text { Dascore Six-Sense } \\
\text { Sonde }\end{array}$ & Specific Conductance, DO, ORP, pH, Temperature and Free Chlorine \\
\hline YSI 6600 Sonde & $\begin{array}{l}\text { Specific Conductance, DO, ORP, pH, Temperature, Ammonia- } \\
\text { Nitrogen, Chloride and Turbidity }\end{array}$ \\
\hline Hydrolab Data Sonde 4a & $\begin{array}{l}\text { Specific Conductance, DO, ORP, pH, Temperature, Ammonia- } \\
\text { Nitrogen, Chloride, Nitrate-Nitrogen and Turbidity }\end{array}$ \\
\hline
\end{tabular}

Source: (Hall et al. 2007) 


\subsubsection{CONTAMINANT SIGNATURE}

Another strategy of using already in place online sensors assumes that the introduction of a specific contaminant would modify the physical and chemical monitoring parameters in a distinct way, which is called the signature of the contaminant. This method is more complex and contaminant signatures would need to be developed. Thus, this strategy is limited to known contaminants. Emerging or unknown contaminants would be determined by the state of the water strategy described above (EPA 2005).

As mentioned in the previous section, the WATERS centre investigated the use of a selection of online sensors to identify specific contaminants and found that general contamination could be determined but signatures proved to be more elusive.

The use of contaminant signatures to identify contaminants in water has been shown to be effective by GardianBlue ${ }^{\mathrm{TM}}$ Early Warning System developed by Hach technologies (for a description of this system see Section 2.2.5). However, this system requires the purchase of Hach instruments and thus eliminates the benefit of using sensors that are already in place.

The use of contaminant signatures to identify contamination is a promising strategy where further research is required to enable the use of any system of online water quality monitoring sensors.

\subsubsection{EMERGING TECHNOLOGIES}

Current single and multi-parameter sensors are widely available and utilize established technology; however, there is always a need to improve sensors in order to make them more efficient, more reliable, or to incorporate advancements in technologies. 
A research team at the Warsaw University of Technology in Poland designed and tested a multi-parameter sensor that used fibre optics to measure $\mathrm{pH}$, temperature and calcium iron concentration as an indication of hardness. The probe used light emitting diodes at three different wavelengths (one for each parameter): $560 \mathrm{~nm}$ for calcium, $630 \mathrm{~nm}$ for $\mathrm{pH}$ and $650 \mathrm{~nm}$ for temperature. Each of the three sensors was in a separate compartment and housed in Teflon. The temperature sensor used a temperature sensitive membrane obtained from polyHEMA hydrogel and was protected with silicone from water evaporation (Dybko et al. 1998).

In 2008 , the same research group presented a second multi-parameter sensor design that utilized optical fibres to measure $\mathrm{pH}$, concentration of calcium ions and total concentration of heavy metals. This device immobilized a series of indicators using chemical bonding on polymeric beads. The indicators used included neutral red for $\mathrm{pH}$ values from 6.5 and 7.8 , while bromothymol blue was used for $\mathrm{pH}$ values from 7.7 to 9.4 . Chloroposphonazo III was used to indicate the calcium concentration and was sensitive to concentrations between $10^{-5} \mathrm{M}$ and $10^{-3} \mathrm{M}$. The indicator for total heavy metal concentration was 4-(2-pirydylazo) resorcinol, which is sensitive at concentrations between $10^{-7} \mathrm{M}$ and $10^{-4} \mathrm{M}$. The beads were placed in a glass tube through which water was allowed to flow. The glass tubes were illuminated with optical fibres and independent LED lights were used to measure each parameter at specific wavelengths: $590 \mathrm{~nm}$ or 630 $\mathrm{nm}$ for $\mathrm{pH}$ (depending on the $\mathrm{pH}$ range), $680 \mathrm{~nm}$ for calcium and $510 \mathrm{~nm}$ for heavy metal ions (Dybko et al. 1998).

A flow through configuration would allow for the continuous online reading of water quality parameters. To assure the sensitivity of each parameter being measured, the 
sensors need to be exchanged and regenerated every three months if used continuously (Dybko et al. 1998).

\subsubsection{AVAILABLE TECHNOLOGY}

GardianBlue $^{\mathrm{TM}}$ Early Warning System and PipeSonde ${ }^{\mathrm{TM}}$ by Hach Corporation, Loveland, Colorado (USA)

www hach.com

Hach Corporation manufactures and distributes analytical instruments, test kits and reagents to test the quality of water and wastewater. Hach manufactures the GardianBlue $^{\mathrm{TM}}$ Early Warning System, which detects, alerts, and classifies a wide variety of contaminants including cyanide, anthrax, arsenic and pesticides. The system is also able to detect and classify events such as water main breaks, caustic overfeeds to cross connection and aging infrastructure problems such as corrosion.

GardianBlue $^{\mathrm{TM}}$ Early Warning System is comprised of an instrument panel which includes: a TOC analyzer, an automatic sampler, and an event monitor. The water panel is a multi-parameter instrument that is able to measure free or total chlorine, conductivity, $\mathrm{pH}$, turbidity, temperature and pressure. Total organic carbon is also measured with a TOC analyzer. Every 60 seconds the water is analysed and compared to baseline data via the event monitor. An alarm is triggered if a user-set threshold exceeds the baseline data. Events are then compared to fingerprints stored in the device's library and a second alert is given if a match is found. If no match is found, the event is stored in the library for future reference. A digital signal transmits the data between the water panel and the event monitor and finally the automatic sampler will collect a sample from the water if an event is triggered to allow for more detailed analysis. 
Hach also manufactures a multi-parameter probe, the PipeSonde ${ }^{\mathrm{TM}}$. Designed to be placed directly in a water distribution pipe, this sensor measures $\mathrm{pH}$, ORP, conductivity, turbidity, DO, line pressure and temperature. Data is also available remotely through the supervisory control and data acquisition (SCADA) system. To perform similar operations as the GardianBlue ${ }^{\mathrm{TM}}$ system, a water distribution panel, a TOC analyzer and an event monitor trigger system can be added. The water distribution panel, coupled with the event monitor trigger system provide real-time analysis of the state of the water and scans for contamination.

\section{F-50 and D-50 Multi-parameter Water Quality Meters by HORIBA (Oakvill, Ontario) \\ www.horiba.com/scientific}

HORIBA manufactures analytical instruments for a variety of markets including the automotive, biotechnology, environmental, medical, semiconductors, energy, pharmaceuticals and food services. HORIBA has a series of portable single parameter sensors that measure: $\mathrm{pH}, \mathrm{DO}$, conductivity and ions such as sodium, potassium and nitrate. The company also manufactures bench-top (the F-50) and a portable (the D-50) multi-parameter water quality devices. The bench-top device allows the measurement of temperature, $\mathrm{pH}, \mathrm{ORP}$, ion concentrations, conductivity and resistivity while the portable device allows for the measurement of temperature, $\mathrm{pH}$, conductivity, ORP and DO. 


\section{Hanna Instruments (Laval, Québec)}

www.hannacan.com

Founded in 1973, Hanna Instruments manufactures and supplies a series of portable single and multi-parameter as well as field test kits and pocket testers for the environmental testing industry. The company is also equipped with research and development (RandD) facilities.

Single parameter sensors are available to measure DO, turbidity, conductivity, colour. Multi-parameter sensors can measure $\mathrm{pH}$, TDS, conductivity and temperature or $\mathrm{pH}$, ORP, conductivity and temperature.

Multi-parameter Device by Emerson Process Management (Columbus, Ohio, USA) www.emersonprocess.com/raihome/liquid

Under the Rosemount Analytical brand, Emerson Process Management produces a multiparameter sensor called the Model WQS Multi-Parameter Electrochemical/Optical Water Quality System. This device is able to measure $\mathrm{pH}$, conductivity, ORP, DO, free chlorine and monochloramine using electrochemical methods, turbidity and particle index using optical methods. The user can also select what combination of water quality parameters, if not all, to be included in the system. No reagents are used with this device, which is able to monitor water quality continuously. 


\section{Six-CENSETM sonde by Censar Technologies (Sarasota, Florida, USA)}

www.censar.com

Six-CENSETM is a multi-parameter in-line sensor able to monitor six parameters: chlorine (free chlorine), monochloramine or dissolved oxygen, $\mathrm{pH}$, conductivity, oxidationreduction potential and temperature. The instrument utilizes electrochemical technology that is placed on a small ceramic chip. The instrument is robust and can be placed directly into pressurized water mains - no reagents required. Data can be remotely downloadable.

The Mohawk Valley Water Authority (New York, USA) operates a large drinking water distribution system to 125,000 people. The system is considered vulnerable to intentional contamination because it includes two open finished water storage reservoirs. As part of increased security measures, Six-CENSE ${ }^{\mathrm{TM}}$ sonde has been strategically placed along the distribution system. The increased security measures also include such basic steps as site security and regular patrols (Schreppel et al. 2002).

\section{YSI 6600 V2 sonde by YSI Incorporated (Yellow Springs, Ohio, USA) WWW.YSI.com}

YSI $6600 \mathrm{~V} 2$ monitoring instrument is equipped with four optical ports available to measure conductivity, temperature, $\mathrm{pH}$ and OPR, as well as DO, turbidity, chlorophyll and blue-green algae. Measurements are done in situ and the instrument is able to record for up to 75 days in 15-minute intervals (limited by the life of the battery). The instrument can also calculate total dissolved solids, resistively and specific conductance. Each optical sensor is equipped with automated wipers to clean the lenses to prevent biofouling. 


\subsection{Monitoring Sum Parameters with Optical Sensors}

The variety of organic pollutants is vast and can include chemicals such as solvents as well as pesticides and their metabolites. Typically, the detection of organic pollutants involves laborious laboratory techniques and specialized equipment such as high-pressure liquid chromatography (HPLC), gas chromatography (GC) and mass spectroscopy (MS).

Sum parameters, such as total organic carbon (TOC), biochemical oxygen demand (BOD), chemical oxygen demand (COD), dissolved oxygen (DO), dissolved organic matter (DOM) and dissolved organic carbon (DOC) are able to give insight into the overall quality of the water. For example high levels of TOC may indicate high humic levels, which can lead to bacterial growth in the distribution system.

Water samples are able to absorb light, which can then be correlated to the magnitude of certain parameter present in the water. The adsorption of different wavelengths of light are associated with different characteristics. For example, dissolved organic matter has been shown to have a good correlation with a wavelength of $254 \mathrm{~nm}$ and five-day biochemical oxygen demand $\left(\mathrm{BOD}_{5}\right)$ has been correlated to a wavelength of $440 \mathrm{~nm}$ (Ahmad and Reynolds 1999). Biochemical oxygen demand (BOD) is a measure of the amount of oxygen needed by microorganisms to degrade organic matter present in the water. Laboratory tests can take up to 25 days, though the five-day test called $\mathrm{BOD}_{5}$ is widely used (Schwarzenback, Gschwend, and Imboden 2002). The uses of optical methods, described below, are useful in reducing the time required for BOD analysis approximately to 20 minutes. 


\subsubsection{UV-VIS SPECTROSCOPY}

UV-VIS spectrometer is a common spectrophotometer that uses the entire UV and visible light regions (200 $\mathrm{nm}$ to $750 \mathrm{~nm}$ ) to determine multiple parameters simultaneously.

A submersible spectrometer UV-VIS spectrometer, utilizing a xenon light source, was used to monitor and control the use of a sequencing batch reactor during wastewater treatment (Langergraber et al. 2004). The device successfully measured nitrate and total suspended solids (TSS) concentrations simultaneously with a $98 \%$ and $99.5 \%$ correlation respectfully. It was installed directly on the reactor wall and real-time data was measured in 15 second intervals. This device has the advantage that no chemicals or sample manipulations are required. Fleischmann et al. (2002) used the same device to monitor the water quality in groundwater while, Kawamura (2003) used the UV-VIS spectrometer to monitor reactions in high temperature conditions.

\subsubsection{Available Technology}

s::can Liquid Monitoring Systems by Aquatic Life Ltd. (Pinawa, Manitoba) www aquaticlife.ca

Aquatic Life Ltd has a multi-parameter UV-VIS spectrometer system that is able to measure turbidity, $\mathrm{BOD}, \mathrm{COD}$, and nitrates in real-time and provide data online. 


\subsubsection{High PERFoRMANCE SIZE-EXCLUSION CHROMATOGRAPHY (HPSEC) AND UV ABSORBANCE (UVA)}

Dissolved organic matter (DOM), or dissolved organic carbon (DOC) is "the organic material that passes a filter, commonly having pore size near $1 \mu \mathrm{m}$ " and is a mixture of: humic and fluvic acids, other acids with low molecular weights, carbohydrates, proteins, and other compounds (Schwarzenback, Gschwend, and Imboden 2002). It is a heterogeneous mixture that can adsorb chemical species in water but also causes taste and odour concerns in drinking water. DOM is generally removed from drinking water during treatment.

DOM contains both light emitting (fluorophoric) and light absorbing (chromophoric) compounds. Because of this, traditional analysis of DOM includes the use of light absorption and fluorescence techniques.

High performance size-exclusion chromatography (HPSEC) is able to provide insight into the composition of DOM as retention time in the chromatography is directly related to the size of a molecule. HPSEC is often coupled with UV-VIS or UV absorbance (UVa) spectrometer (Nollet 2007).

Aromatic compounds, such as polysaccharides and proteins, have recently been shown to cause fouling in nanofiltration systems and must be removed from the water. To effectively remove these compounds, it is first required to quantify them. Her et al. (2002) modified a commercially total organic carbon (TOC) analyzer to improve the detection of DOM as a function of molecular weights. The modified instrument was used in conjunction with a HPSEC-UVa system. All instruments were connected together and fed with a pump. Data could be displayed in real-time. 


\subsubsection{FLUORESCENCE SPECTROSCOPY}

Fluorescence spectroscopy is an optical technique that uses three-dimensional excitationemission matrices recorded at two wavelengths. Fluorescence measurements are affected by environmental factors (temperature, $\mathrm{pH}$, ect.) and are not suitable for in situ measurements (Nollet 2007).

On the other hand, synchronous fluorescence spectroscopy is a two-dimensional technique that requires a single scan where excitation and emission wavelengths are measured at the same time. Hur et al. (2008) used synchronous fluorescence spectroscopy and found an excellent positive relationship with BOD in river water samples.

\subsection{MONitoring Microbiological CONTAMinantS}

A biological contaminant is any living organism that can negatively affect the health of the public or receiving body of water. Testing of water for biological contaminants is traditionally done using culture methods, such as the Heterotrophic Plate Count (HPC), where indicator organisms are cultivated on a selective media and the resulting number of colonies is counted. Depending on the type of agar used and organism selected for, a plate count takes a minimum of 24 hours and requires the use of laboratory equipment (e.g. ovens and incubators). In addition, if colonies are grouped or difficult to distinguish the count is prone to human error.

An Indicator organism is defined as a non-pathogenic organism that is easy to isolate and is present in polluted waters but not treated waters (Organisation for Economic Cooperation and Development (OECD) and (WHO) 2003). Indicator organisms are selected because they are easy to culture and provide insight as to the microbial quality of the water. A common indicator organism, bacteria Escherichia coli (E.coli), is typically 
found in feces and its presence in water is a good indication of fecal contamination. However, the absence of E.coli does not confirm the presence of more resistant viruses or protozoa. The use of bacterial indicators is problematic because it may not reflect the presence of other microorganisms in the water.

In the past 20 years, new pathogens have emerged causing acute problems in humans and raising level of concern. These pathogens include but are not limited to Cryptosporidium, Legionella, Campylobacter, Yersinia, Mycobacterium avium complex (Mac), Aeromonas hydrophila and Helicobacter pylori (Health Canada 2006). There has also been an increased awareness of the vulnerability of water distribution networks as a vehicle for bioterrorism. An act of bioterrorism is the use of a living organism to cause harm, and typically a human disease causing strain of a bacteria virus or protozoa is employed (Paul 2003).

Candidates for a bioterrorism agents are contagious, have a small infectious dose, are stable and will disperse in water, cause severe disease that is difficult to cure and are resistant to chlorine. The Emergency Preparedness and Response division of the US Centers for Disease Control and Prevention (CDC) has published a list a bioterrorism agents of concern. The list is separated into one of three categories (A, B and C) based on the level threat. Category A agents, including anthrax, botulism toxins and smallpox, are easily disseminated, can cause harm to a large amount of people and have high mortality rates. Category B agents, such as E.coli, Cryptosporidium and mycobacterium tuberculosis, are moderately easy to disseminate and cause moderate mortality rates. Category $\mathrm{C}$ agents include emerging pathogens that have the potential to be developed 
into a threat due to ease of production. An example of the latter category includes the hantavirus. (CDC)

In light of increased threats, there is a need to escalate the level of protection concerning water supplies and distribution systems. More rapid molecular techniques are needed to be able to detect a large variety of microorganisms quickly in order to mitigate the effects of the contaminant by treating the water and advising the public of the threat (OECD and WHO 2003).

New monitoring technologies should: (1) require no sophisticated laboratory equipment, (2) require minimal sample preparation, (3) detect an array of pathogens (not only a single pathogen) and (4) differentiate between viable and non-viable pathogens. Sensors for living organisms typically target genetic material (nucleic acid), proteins, or adenosine triphosphate (ATP). Most sensors based on biological interactions have biological components and detection is performed by various methods such as light production or mass change. Concentration of the target molecule is typically needed. 


\subsubsection{MICROBIOLOGICAL CONTAMINANTS OF CONCERN}

There are a number of possible microbiological contaminants that have been discussed in the literature, some of which will be discussed here; for a summary, see Table 2.4.

\subsubsection{Anthrax}

The Bacillus antracis spore can be found in nature but is also available in weapons grade form. Anthrax is most known from the 2001 postal service attacks that killed six people and infected another 22 in the US. While dispersion of anthrax in the air is most probable, the spores are stable in water for up to two years and are resistant to chlorine.

Anthrax can be contracted through inhalation, skin contact (especially through wounds) or ingestion. Infections resulting in death are caused by a build-up of toxin in the bloodstream (toximia) and suffocation. It is estimated that there are 100 billion to 1 trillion spores per gram of anthrax. The infectious dose of anthrax can be as low as 1 to 3 spores or as high as 2,500 to 5,000 . While there is a vaccine available, it has been formulated for naturally occurring anthrax and may not be effective for the weapons grade variety (Paul 2003; States et al. 2002).

A conventional treatment train would reduce but not eliminate the spores in the water. However, adding filters such as microfiltration, ultrafiltration, nanofiltration or reverse osmosis to the treatment train would be effective at removing the spores. Knowing this, the most effective use of anthrax in terms of a Bioterrorism agent would be in the distribution system. Polymerase chain reaction (PCR) techniques can positively identify anthrax spores (see Section 2.4.3) (Paul 2003). 


\subsubsection{Botulinum Toxin}

The bacteria Clostridium botulinum is naturally found in soil, as well as marine and lake sediments. The toxins produced by this bacteria cause botulism; a disease that blocks nerve signals to the muscles and can cause death. Botulism can be contracted by ingestion, inhalation or infection of wounded skin. The lethal dose of the botulinum toxin varies depending on the pathway into the human body. If ingested approximately $70 \mu \mathrm{g}$ would be needed, however if injected intravenously only approximately $1 \mathrm{ng}$ would be needed (Paul 2003;Arnon et al. 2001).

Spores can survive in boiling water for up to four hours, and up to 1.5 hours in water at a temperature of $105^{\circ} \mathrm{C}$. The spores are also resistant to UV radiation but both the spores and toxins can be killed with chlorine. Due to dilution, it is unlikely that the toxins would be introduced in water because a large amount would be needed. In addition, spores are naturally deactivated within 3-6 days (Paul 2003).

Antitoxins are available for different strains of the bacteria; however, they are only effective on toxins that are not bound to nerve cells. For this reason, the antitoxins are most effective if administered within 24 hours of infection (Paul 2003).

\subsubsection{E.coli}

Escherichia coli (E.coli) are naturally found in the intestines of warm-blooded mammals, which includes human. While most of the several hundred strains are harmless, the strain O157:H7 produces a toxin that can cause illness such as abdominal pain, bloody diarrhoea, haemolytic-uremic syndrome (HUS) and death. Most often, illness from this strain is caused by eating undercooked beef. E.coli is used as an indicator of fecal coliform contamination of the water (Health Canada 2006). 
E.coli are stable in water that contains a moderate microflora at temperatures between 15 and $18{ }^{\circ} \mathrm{C}$ for a duration of 4 to 12 weeks. While the bacteria can survive, water is not a suitable environment for the bacteria to grow. Conventional treatment that includes filtration and disinfection is typically effective at removing E.coli. A variety of disinfectants are suitable including chlorine, chloramines, chlorine dioxide, UV light and ozone. To maintain a safe drinking water in the distribution system, a chlorine residual is often applied.

\subsubsection{Cryptosporidium parvum}

Cryptosporidium parvum is a protozoan which agglomerates to create oocysts that measure 4 to $6 \mu \mathrm{m}$ in diameter. Due to the protection of an outer shell of the oocysts, C.parvum is resistant to chlorine, can withstand freezing, salt water and can live in cold water for up to 18 months. It naturally lives in the human and animal and can be transmitted through fecal contamination by an infected person. Immunosuppresed individuals are particularly susceptible to infections (Health Canada 2006).

To remove C.parvum, water needs to be boiled or filtered. C.parvum cannot be cultured. Monitoring technologies are only semi-quantitative and do not provide information on the protozoan's viability or human infectivity. Concentration techniques are also needed (Health Canada 2006).

\subsubsection{Giardia lamblia}

Giardia lamblia is a flagellated protozoan that naturally lives in the small intestines of humans and other animals. It can cause acute gastrointestinal problems with as little as 1 to 10 cysts and can cause kidney damage or death to immune-compromised individuals. Once in water, G.lamblia will agglomerate to form cysts measuring approximately $10 \mu \mathrm{m}$ 
in diameter. These cysts can survive for up to 77 days at $8^{\circ} \mathrm{C}$ and survival rates decrease with increasing temperatures. The cysts are resistant to disinfection; however a multibarrier approach, which includes filtration and disinfection, is effective at reducing the concentration of this protozoan (Health Canada 2006).

G.lamblia is not able to be cultured. Routine methods of detection require concentration techniques, account for only a fraction of the total number of microbe present and provide no information on viability or the ability to infect humans. E.coli is used as a surrogate parameter to indicate the possible presence of G.lamblia.

\subsubsection{Plague}

Plague is caused by the bacteria Yersinia pestis. There are two types of plague, bubonic and pneumonic, which differ in their method of transmission. The bubonic plague, also known as the Black Death, can be harboured in animals and is typically transmitted through animal bites or scratches. Infections cause swelling of the lymph nodes, and if left untreated, the bubonic plague has a 50\% mortality rate (Paul 2003).

The pneumonic plague is rarer but can develop from complications of the bubonic plaque. The former affects the lungs and is transmitted and dispersed in air through coughing and sneezing. Approximately 100 to 500 cells are required to cause infections and are thus easily spread from person to person. While both forms of the plague are stable in water for several days, there have been no reports of infection by ingestion (Paul 2003). 
ก

\begin{tabular}{|c|c|c|c|c|c|c|c|c|c|c|}
\hline & & 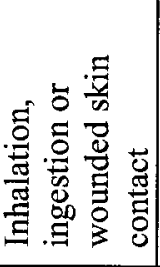 & 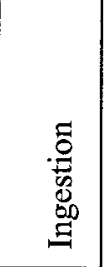 & 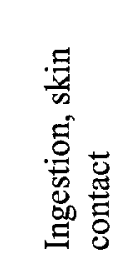 & 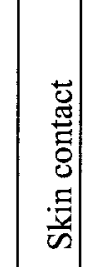 & 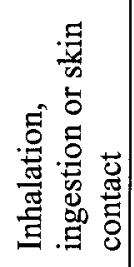 & & & & \\
\hline & . & 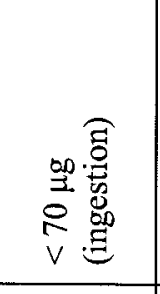 & 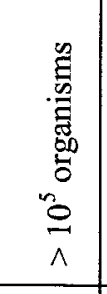 & $\frac{\pi}{z}$ & | & 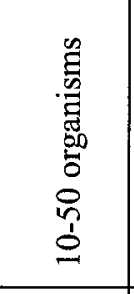 & 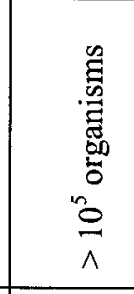 & 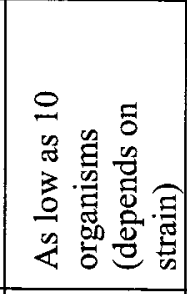 & 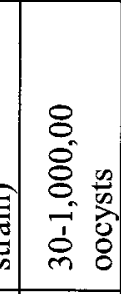 & s. \\
\hline & 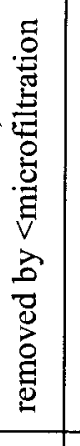 & 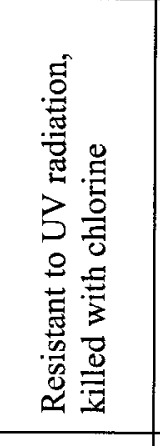 & 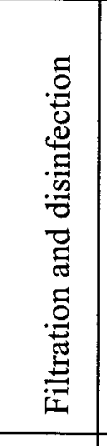 & 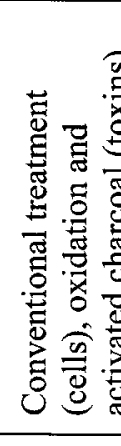 & & & 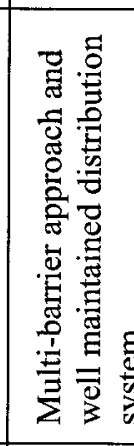 & 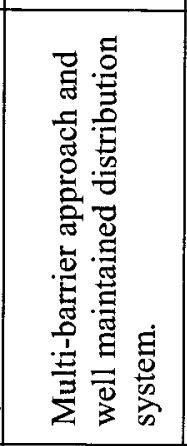 & 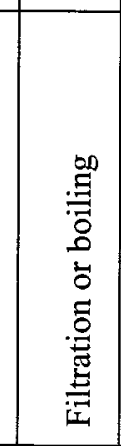 & 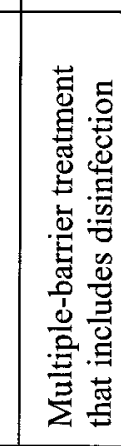 \\
\hline & 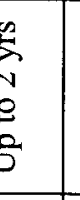 & $\begin{array}{l}\text { 堀 } \\
\text { 号 } \\
\end{array}$ & $\frac{7}{b} \frac{0}{b}$ & 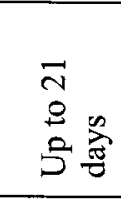 & 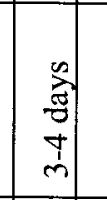 & & & & & \\
\hline & 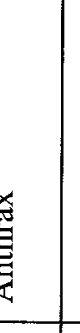 & 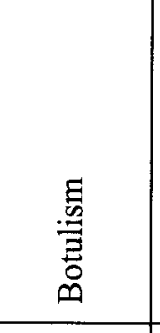 & 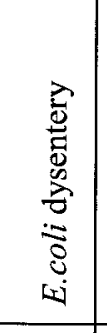 & 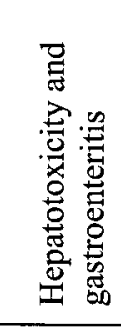 & 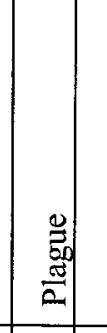 & 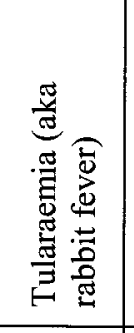 & 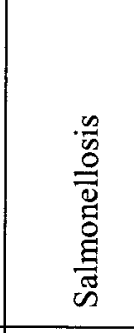 & 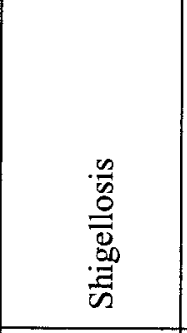 & 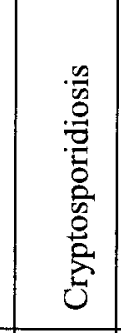 & 爮 \\
\hline & 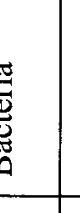 & 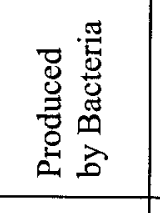 & 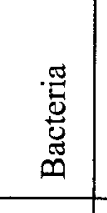 & 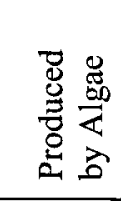 & 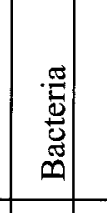 & 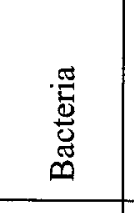 & 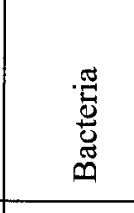 & 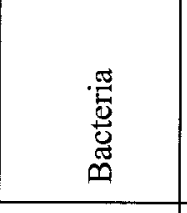 & 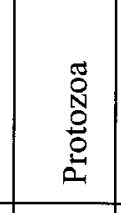 & 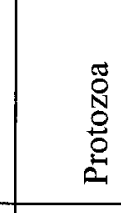 \\
\hline & 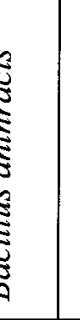 & 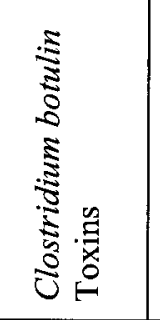 & 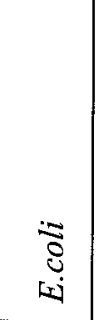 & 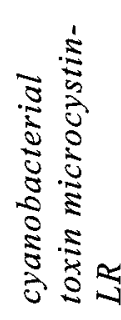 & 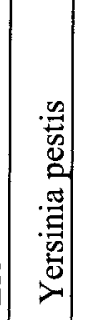 & 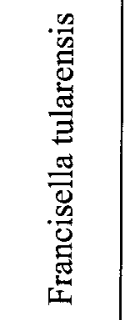 & 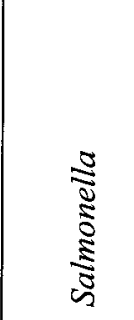 & 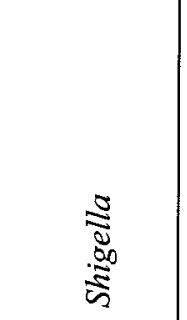 & 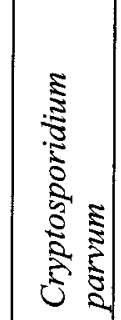 & 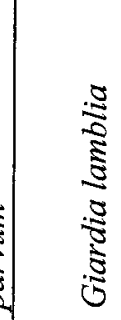 \\
\hline
\end{tabular}




\subsubsection{IMMUNOASSAYS}

Immunoassays are typically performed on grab samples and are based on antigenantibody reactions. A positive immunoassay will produce a visible light or colour. An immunoassay requires a capture and target molecule. A capture or recognition molecule is the part of the sensor that interacts directly with the sample, and can be either antigens or antibodies. A target molecule of the microorganism interacts with the capture molecule of the sensor. Secondary antibodies are added to link with an enzyme. Immunoassays are easy to use and require little specialized knowledge. However, they are prone to falsepositive responses as molecules other than the target molecules may react with the capture molecules (EPA 2005).

A strip test or later flow assay is a common type of immunoassay used to detect antigens in water. A common example of a lateral flow assay is the home pregnancy test. For this assay, an adsorbent membrane is fixed on a solid piece of plastic called the strip and each $\underset{*}{\operatorname{mambrane}}$ can have a number of different stripes. Each stripe contains high concentrations of a specific antibody labelled with either a coloured dye or a fluorescent agent. A liquid sample is applied to one end of the strip and allowed to diffuse. A colour change or fluorescence response of a stripe within the strip indicates the presence of an antigen in the sample. A control stripe is often placed at the end of the strip to indicate the completion of the test. These tests are rapid, with results typically obtained within 15 minutes, although they provide no quantitative information (EPA 2005). There is a number of readily available lateral flow assays available to detect the presence of common bio-warfare agents. 
These assays have relatively high levels of detection. For example, the detection limit of the Bio Threat Alert $($ assay produced by Tetracore for the detection of anthrax is 100,000 $\mathrm{CFU} / \mathrm{mL}$. Some manufactures have reported that detection limits can be reduced to $10,000 \mathrm{CFU} / \mathrm{mL}$ if an electronic reader is used. Thus, lateral flow assays for the detection of biological contaminants in water are not suitable for trace elements but more for large scale contamination events or for preliminary screening purposes. Dilution of the contaminant in water may also reduce the effectiveness of the assays. The confirmation of contamination should be performed by more precise measures (Casson et al. 2006). Concentration of a water sample may improve the limits of detection. Currently in development is an assay that uses ultrafiltration techniques to concentrate $1,000 \mathrm{~L}$ to 250 $\mathrm{mL}$ increasing the detection limit to $25 \mathrm{CFU} / \mathrm{mL}$. Concentrating samples with centrifuges is also possible but requires additional research (Casson et al. 2006).

\subsubsection{Available Technology}

\section{RAMP Anthrax Assay by Response Biomedical Corporation (Vancouver, British Columbia)}

www responsebio.com

RAMP Anthrax Assay uses a portable fluorescent reader to read the response of test cartridges exposed to a water sample. The cartridges are lateral flow immunoassays using fluorescent secondary antibodies and equipped with a test and a control stripe. Assays are available to detect anthrax, botulinum toxin, ricin, and smallpox. Results are produced within 15 minutes. This assay has been used by U.N. biological weapons inspectors but has not been tested for the use in drinking water. 


\section{Bio Threat Alert (i by Tetracore (Gaithersburg, Maryland, USA)}

www tetracore.com

Bio Threat Alert $®$ produces a reddish band, visible to the naked eye, in the presence of specific contaminants (a list of contaminants for which this test is available for, as well as associated detection limits are presented in Table 2.5). To perform the test, a couple drops of a sample is mixed with a buffer solution and allowed to diffuse on the test strip.

Table 2.5: Contaminants to be detected by Bio Threat Alert $\mathbb{B}($ EPA 2005)

\begin{tabular}{|l|r|}
\hline Contaminant & Detection Limit \\
\hline Bacillus anthracis & $1 \times 10^{5} \mathrm{CFU} / \mathrm{mL}$ \\
\hline Yersinia pestis & $2 \times 10^{5} \mathrm{CFU} / \mathrm{mL}$ \\
\hline Francisella tularensis & $1.4 \times 10^{5} \mathrm{CFU} / \mathrm{mL}$ \\
\hline Botulinum toxin & $10 \mathrm{ppb}$ \\
\hline Staphylococcal endotoxin & $2.5 \mathrm{ppm}$ \\
\hline Ricin & $50 \mathrm{ppm}$ \\
\hline
\end{tabular}

\section{SMART ${ }^{\mathrm{TM}}$ by New Horizons Diagnostics (Columbia, Maryland, USA)}

www nhdiag.com

Sensitive Membrane Antigen Rapid Test or SMARTTM is a lateral flow assay that uses gold-labelled antibodies on a membrane. One test is equipped with two stripes, one to test for the contaminant and one for the control. The SMART'M is able to detect Vibrio cholera 01 with a detection limit of $10^{5} \mathrm{CFU} / \mathrm{mL}$ for bacteria and $50 \mathrm{ppb}$ for biotoxins. The test will produce results within 15 minutes and also requires a couple of drops of a sample to be mixed with a buffer solution. 


\section{BADD $^{\text {TM }}$ and Pro Strips ${ }^{\text {TM }}$ by ADVNT (Phoenix, Arizona, USA)}

www advnt.org

Bio-Warfare Agent Detection Device or BADD ${ }^{\mathrm{TM}}$ has been used by U.N. weapons inspectors in Iraq. It is a qualitative test that screens for anthrax, botulium and ricin toxin, and Yersinia pestis (bacteria). Two stripes are used to indicate a positive response and a positive control. The stripes are visible to the naked eye and results are produced in three to fifteen minutes. Pro Strips ${ }^{\mathrm{TM}}$ is a handheld cartridge that allows for all five tests to be performed with one sample.

\subsubsection{Emerging Technology}

Quantitative Lateral Flow Assay by NASA's Jet Propulsion Laboratory (Pasadena, California, USA)

Technology is currently being developed to produce quantitative lateral flow assays to determine the concentration of a certain bacteria in water. For example, NASA's Jet Propulsion Laboratory has developed a quantitative test strip to test water for E.coli in space. Using chemical markers as secondary antibodies, a positive test strip will produce different colour intensities depending on the concentration of the bacteria present in the water. This type of test is promising for the future because quantitative results can be obtained within minutes without the growth of bacterial populations. 


\subsubsection{Conventional AND REAL-TImE QUANTITATIVE Polymerase ChaIN REACTION}

Polymerase chain reaction (PCR) is an example of a rapid testing method that is able to detect microorganisms, including bacteria, protozoa, and even viruses that are not able to be cultivated. PCR allows for the amplification of a target sequence of microbial DNA so that it can be easily detected. The choice of the primer, a specific DNA sequence, allows for the selection of different strains, species, and genus of a microorganism. PCR amplifies the selected DNA sequence so that it can be easily detected (Dorigo, Volatier, and Humbert 2005).

To complete a PCR method, the temperature is cycled in three steps: (1) at $94^{\circ} \mathrm{C} \mathrm{DNA}$ is denatured into two single strands, (2) at $60^{\circ} \mathrm{C}$ primers are annealed to the single-stranded target DNA sequence, and (3) at $72^{\circ} \mathrm{C}$, DNA polymerase is elongated and a new strand of the target DNA is created. Several hundred copies of a target DNA can be created in an hour (Casson et al. 2006; States et al. 2002).

PCR amplification can be initiated with as little as one strand of DNA. This allow for lower detection limits compared to other detection methods. Detection of biological warfare agents and human viruses depends on the selection of a primer. Detection of human viruses is especially important because viruses are species specific. Human virus present in the water indicates contamination by a human fecal source. PCR is also a rapid detection method that can be completed in a few hours and is more sensitive than culture based methods. 
However, PCR is not without its disadvantages. Amongst them, this method requires specialized knowledge, specialized laboratory conditions and equipment. In addition, prior to its completion, microbial DNA must be extracted using a cell lyses procedure and post-processing techniques such as gel-electrophoresis and photo-documentation are also required. PCR qualitatively determines if a certain DNA target is present, however; since DNA is able to survive after the death of a cell, PCR is not able to determine the viability of the DNA identified (OECD and WHO 2003; States et al. 2002; Zhou et al. 2007).

Improving PCR, real-time quantitative PCR (RT-PCR) allows for the quick determination of DNA and RNA using fluorescent DNA-binding probes, such as the TaqMan probe. Compared to conventional PCR, RT-PCR is able to reduce the time needed to produce results from three hours to less than one. While the pre-processing step of DNA extraction is still required, post processing steps are eliminated.

During RT-PCR, fluorescence increases as the reaction progresses and more of the targeted DNA, or amplicon, is amplified and fluorescence is recorded during each amplification cycle (approximately 5 seconds per cycle) during the exponential growth phase. The cycle number at which amplification reaches a concentration threshold is called the $C_{t}$ value which is inversely proportional to the quantity of DNA in solution. Using calibration curves formulated with known amounts of DNA, one can determine the amount of DNA present in solution. The greater the amount of template detected at the beginning of RT-PCR then, fewer cycles are needed to reach the detection levels (Dorigo et al. 2005; Zhou et al. 2007). 
Compared to conventional plate count methods, RT-PCR has higher sensitivity, precision, and reliability. It is independent of culture conditions, nutrient conditions and bacterial metabolic states. It also has the ability to process more than one reaction at once and is able to quantify microbial DNA including viral DNA, as well as RNA and mRNA (Dorigo et al. 2005; States et al. 2002; Schmitten 2001; Zhou et al. 2007).

When using RT-PCR, primer selection is important. While the method favours short amplicons and requires high specificity, universal primers are not very specific and new amplicon formulations are currently being developed. To evaluate the microbial quality of water, Zhou et al. (2007) used conventional PCR to select the most appropriate pair of primers for the identification of E.coli in water and used RT-PCR to quantify the amount of E.coli.

Real-time PCR has been applied to water monitoring to identify human pathogens. For example, Naegleria fowleri was successfully detected with $99 \%$ efficiency using a duplex real-time PCR (Behets et al. 2007) while, Donaldson et al. (2002) developed a real-time reverse-transcriptase PCR method to detect the presence of enteroviruses in surface waters. Zhou, et al. (2006) used real-time PCR to determine total bacterial DNA in water.

Common species of Cyanobacteria in freshwater lakes (such as Anabaena, Microcystis, and Planktothrix) can produce toxic cyclic heptapeptides and microcystins. Vaitomaa et al. (2003) used real-time PCR to identify the microcystin-producing genus of bacteria, Anabaena, and Microcystis, and were able to distinguish between toxic and non-toxic forms. 


\subsubsection{Available Technology}

\section{DX and DX-12 by Spartan® Bioscience (Ottawa, Ontario)} www. spartanbio.com

Spartan $®$ Bioscience has two compact bench-top real-time PCR instruments that differ in the amount of testing wells and temperature programs. The Spartan DX offers four wells and two temperature PCR programs while the Spartan DX-12 has 12 wells and three temperature PCR programs. Both instruments are compact, to maximize bench space and are able to provide results in real-time between 15 and 90 minutes. While these real-time PCR instruments are designed for the use in the lab, they are lightweight, can standalone and are thus somewhat portable. Pathogens such as Herpes Simplex Virus (HAV), Enterovirus, West Nile Virus, E.coli and Tuberculosis can be detected. There is potential for this instrument to be further developed for use in on-line water quality monitoring.

\section{Fermentas International Inc. (Burlington, Ontario)}

www. fermentas.com

Fermentas international is a biotechnology company that specializes in the discovery, manufacturing and marketing of molecular biological products. The company has a number of optimized solutions, primers, buffers and other reagents needed to complete standard PCR and real-time PCR trials. 


\section{Qualicon BAX PCR system by DuPont (Mississauga, Ontario, Canada)}

www.dupont.ca

BAX PCR system was developed by the DuPont-Wualicon Corporation in association with the American Water Works Service Company for the use in routine microbial laboratory analysis. It is an automated system that is able to detect the presence or absence of a certain microorganism within four hours. The BAX PCR has been used successfully by the Pittsburgh Water and Sewer Authority to measure Cryptosporidium parvum, Salmonella and E.coli 0157:H7 (States et al. 2002).

The system eliminates multiple liquid transfer of the sample with the use of a tablet that contains all primers, polymerase, nucleotides, positive control, and fluorescent dyes necessary to complete a test. The facility of a tablet also allows for the processing of up to 96 tests in one batch.

The system also includes an automated thermal cycler and fluorescence detector to facilitate the process and eliminates the post processing steps usually associated with PCR. After approximately three hours of amplification, one hour of detection follows. During the detection phase, the temperature is gradually increased to lower the fluorescence signal by releasing the fluorescence from the DNA strands. A software package is able to correlate the fluorescence change to the change in temperature and determine the presence or absence of certain molecules (States et al. 2002). 


\section{R.A.P.I.D. by Idaho Technology Inc. (Salt Lake City, Utah, USA)}

www idahotech.com

Ruggedized Advanced Pathogen Identification Device (R.A.P.I.D.) is a portable real-time PCR device used to detect biological agents. In particular, it can identify Bacillus anthracis, Campylobacter spp., Francisella tularensis, E.coli, Yeersinia pestis, Salmonella spp., Brucella spp., Listeria monocytogenes and Clostridium botulinum (Botulinum toxin). Results are obtained within 30 minutes and the instrument has a detection limit of $1000 \mathrm{CFU} / \mathrm{mL}$.

\subsubsection{Emerging Technology}

BEADS by Interfacial Chemistry and Engineering Group of the Pacific Northwest National Laboratory

www.pnl.gov

Biodetection Enabling Analyte Delivery System (BEADS) is an automated, flow-through real-time PCR, which includes automated cell-capture micro-columns. Developed by the Interfacial Chemistry and Engineering Group of the Pacific Northwest National Library, the system achieved automated sample collection, purification, amplification, labelling, and detection with a detection limit of 10 E.coli $(\mathrm{O} 157: \mathrm{H} 7)$ using river water samples. The system was also able to detect, simultaneously, E.coli, Salmonella, and Shigella with a limit of 100 cells (Straub et al. 2005).

Primers and probes have been developed to target adenoviruses (viruses found in human feces) and enterococci bacteria respectfully. Viruses are more resistant to disinfection than bacteria and thus the use of the E.coli as indicator bacteria is problematic. The BEADS system allows for the real-time monitoring of viruses. The system is also more sensitive than culture-based methods though some overestimations of viral and bacterial 
cultures was reported. Direct measurement of viral concentrations gives a view of the microbial quality of water.

Though the BEADS system is still in development, it can be field ready in approximately five years. The current cost of the system is estimated to be ten times that of current culture techniques. While false positives were not observed, false negatives are possible if the microbial concentration is below the detection limit of the system. In addition, the system is prone to overestimate the pathogen quantities. As with all real-time PCR techniques, the technology identifies a target DNA sequence but cannot discriminate between viable, non-viable or damaged sequences (Jian 2006).

\section{HANNA by Global Security of the Lawrence Livermore National Laboratory (Livermore, California)}

https:/www-gs.lnl.gov/

The hand-held advanced nucleic acid analyzer or HANNA uses PCR to detect bioterrorism agents. It performs PCR in real time by monitoring the fluorescence generated by reaction between nucleic acid probes and the target DNA sequence. It is small in size and battery operated, thus making it very suitable for field evaluations. The PCR process depends on cycling the temperature between high and low points. To do this, the HANNA instrument uses advanced silicon thermal cycling technology developed by the Lawrence Livermore National Library. Each unit has four thermal cyclers and is able to perform two DNA assays simultaneously. In 1999, 11 HANNA instruments were constructed to for field testing purposes (Benett 2000). 
The Water Environment Research Federation (WERF) evaluated one of the prototype instruments for use in water monitoring for the detection of Cryptosporidium parvum and E.coli. Nucleic acid probes were first specifically developed for the detection of the above mentioned pathogens. Using water that contained E.coli from a beef cattle farm in West Virginia, the HANNA instrument was able to detect the E.coli indicator gene, lac Z, in less than 13 minutes, and a virulence gene in 25 minutes (Jenkins et al. 2001).

While the HANNA instrument allowed for a quick PCR result, it is in need of some improvements. For example, there was a large amount of variability of the real-time fluorescent results and the instrument tested by WERF easily overheated and required replacement within nine months. In addition, while the instrument is equipped with four thermal cycles only two assessments can be performed at one time because controls are needed for each test (Jenkins et al. 2001).

\subsubsection{DNA MICROARRAY}

Conventional pathogen detection methods allow for the detection of one gene, and thus one pathogen per experiment. Deoxyribonucleic acid (DNA) microarray technology allows for the simultaneous detection of hundreds or even thousands of pathogens during one experiment (Brousseau 2004).

A DNA microarray is a series of genes immobilized on a solid surface such as a glass slide or a silicone "chip". Immobilized DNA molecules, also known as probes, are ordered in a pre-determined order. A target is the complementary series of polynucleotides to the probe. Targets will hybridize with its complementary probe. Fluorescent labelling of the target allows for the portion of the array where hybridization 
has occurred to be identified. DNA microarrays are thus able to identify thousands of genes of a small sample simultaneously (Brousseau 2005; Dorigo et al. 2005).

Recent research has been concerned with the identification of microorganisms in more complex matrices. For example, DNA microarray technology has recently been successful in identifying metabolic genes of bacteria in a laboratory-scale sequencing batch-reactor. In total, the microarray contained 64 genes and average four days for analysis (Dennis et al. 2003).

Prior to using DNA technology, DNA must be extracted. To do this, protocols, such as polymerase chain reaction (PCR) amplification have been developed. DNA microarray preceded by an extraction procedure requires a complex laboratory set up, specialized knowledge and a time of two and a half days. Increasing the speed of PCR amplification would improve the efficiency of the process. Reproducibility of PCR amplification between samples and control of background fluorescent hybridization has also been problematic (Brousseau 2005).

With increased research, DNA microarray technology has the potential to be a useful tool for the microbial characterization of water and wastewater including the rapid detection of bioterrorism threats (Brousseau 2005; Dennis et al. 2003). 


\subsubsection{ATP ASSAYS}

The adenosine triphosphate (ATP) assay measures the quantity of ATP and is based on the luminescent reaction between ATP, luciferin and luciferase. Luciferin and luciferase are extracted from fireflies. ATP is a molecule involved in the energy transfer of all living cells. If a cell dies, ATP is quickly decomposed. Light produced from the reaction between luciferin-luciferase and ATP is an indication of the amount of living organisms present in water. Contrary to DNA assays, the ATP assay is a measure of cell viability (Shimomura 2006).

One of the first ATP assays used was developed to detect bacteria for the food and beverage industry. A method developed by Tanaka et al. (1997) required cultivation of bacteria from a food sample on a special membrane filter until there was sufficient amount of ATP. Then, luciferin-luciferase was allowed to react with the ATP and the luminescence of the reaction was detected by an ultra-high sensitive cameral and image analyser. Results were obtained in six to seven hours, whereas a typical plate count requires 24 hours minimum (Tanaka et al. 1997).

Today, many ATP assay kits are available that require only a small sample volume (typically under $20 \mathrm{~mL}$ ) and produce results in 30 seconds to an hour. Kits are available to measure either extracellular or microbial ATP; however, if the latter is being measured it must first be extracted from cells. To do so, a filter is first used to collect bacterial cells and those cells are lysed to release ATP. Most tests are performed onsite and require no sample preparation.

Current research typically compares the results of the ATP assay to culture methods to assess the application of the test to surface water, groundwater, wastewater and water in 
the distribution system. Three limitations of the assay have been identified and are as followed:

1. The ATP assay measures the total microbial ATP; however it is not able to measure the contribution of a particular source.

2. Depending on the assay and methodology used, the lower sensitivity limit can vary; however it needs improvement to be used as a water monitoring.

3. The quantity of ATP per cell may vary depending on species, strain, and environmental and metabolic factors, yet there is currently no simple way to convert quantity of microbial ATP to quantity of bacterial cells.

In any water sample, especially surface water and wastewater, there may be a variety of microorganisms that contribute to the quantity of ATP measured. If an assay does not include a step to eliminate the extracellular ATP from a sample, the quantity of ATP may include cellular and extracellular ATP. To identify the ATP contribution of E.coli in a surface water sample, Lee and Deininger (2004) used immunomagnetic separation (IMS) to isolate E.coli before proceeding with the ATP bioluminescence assay. IMS uses polystyrene beads coated with antibodies that combine with a specific microorganism or group of microorganisms. Once bonded, a magnetic field removes the bead-bacteria particles and a micro-centrifuge is then used to separate the bacteria from the beads. Results of the IMS-ATP procedure were compared to traditional plate counts. The IMSATP procedure tested used between 100 and $500 \mathrm{~mL}$ of sample water and was able to achieve a detection limit of approximately $20 \mathrm{CFU} / \mathrm{mL}$. The antibodies may bind with other enteric bacteria which would overestimation the E.coli in the water. The IMS also concentrated water samples. Although no evidence suggested that plant material or 
suspended particles would interfere with the test, prior to completion of the IMS-ATP assay, water samples were filtered with a $20 \mu \mathrm{m}$ filter. The entire procedure was completed in less than one hour (Lee and Deininger 2004).

IMS-ATP was also verified with the use of wastewater in a paper by Bushon, Likirdopuos and Brady (2009). Reducing the sample volume to $25 \mathrm{~mL}$, researchers were able to achieve a lower detection limit of $10 \mathrm{CFU} / 100 \mathrm{~mL}$ for most but not all samples. It was hypothesized that the antibodies used with the magnetic beads may have been reacting with other organisms in the wastewater sample making the detection limit inconsistent (Bushon, Likirdopulos, and Brady 2009).

The ATP assay has also been tested for drinking water applications. Deininger and Lee (2001) tested drinking water samples from the United States and international sources with the ATP assay and compared the results with those of culture methods as well as more complex and non-standard methods such as Acridine Orange Direct Count and Direct Viable Count. Sample volumes used for analysis with the ATP assay ranged between 0.1 and $10 \mathrm{~mL}$. There was a strong correlation between the plate count and ATP assay; however, the ATP assay was limited by a lower detection limit of 200 cells. Due this limitation, it was suggested that the ATP assay could be used to monitor for bioterrorism of microorganisms with an infection dose of one million cells in distribution networks or reservoirs. (Deininger and Lee 2001)

In a separate study, the same authors suggested the use of the ATP assay for the detection of wastewater contamination of a distribution system (Lee and Deininger 2010). Raw wastewater, which contains high levels of microorganisms, would be easily detected by a 
spike in the quantity of ATP measured by the ATP assay in a distribution network (Lee and Deininger 2010). Similarly, Delahaye et al, (2003) evaluated the use of the ATP assay in the distribution network of Paris. Due to low linearity between the plate count and the ATP assay that were found, this study recommended the use of the ATP assay as an indication of microbial drift or as a contamination detection tool but was unsuitable to determine water quality (Delahaye et al. 2003).

A number of procedures have been suggested to increase the sensitivity limits of the assay. For example, Cho and Yoon (2007) tried three concentration methods (amplification by culturing, concentration with membrane filtration and enzymatic method) to improve an initial sensitivity limit of $10^{4}$ to $10^{5}$ cells per vial. Of the three methods, amplification by culturing for 4-hours was the preferred method and achieved $10^{2} \mathrm{CFU} / \mathrm{mL}$ (Cho and Yoon 2007). The improved method was verified with chlorine and UV disinfection experiments and showed good correlation with the spread plate culture method for 3-log reduction of bacteria. The UV inactivation experiments were completed with doses of 10,20 and $30 \mathrm{~mW} / \mathrm{cm}^{2}$ and the type of UV lamps used were not specified (Cho and Yoon 2007).

In a separate study, Hames et al. (2010) heated the samples to $38^{\circ} \mathrm{C}$ before testing in an effort to increase the sensitivity limit. It was hypothesized that since the ATP assay depends on the enzymatic reaction between luciferin and ATP, increasing the temperature would increase the performance of the assay. Doing so increased the performance of the assay between 12 and 16 times and reached a lower sensitivity limit of $0.0001 \mathrm{nM}$ ATP (or approximately $5 \times 10^{-3} \mathrm{ng} \mathrm{ATP} / 100 \mathrm{~mL}$ ). This study found a low linearity between the ATP assay and Heterotrophic Plate Counts (HPC) using natural water and wastewater 
effluent samples. These results were not surprising because the microbial population in the aforementioned samples have a diverse bacteria population. Good correlation of the ATP assay was observed when compared to viable cells measured by flow cytometry after staining with propidium iodide (Hammes et al. 2010).

The concentration of ATP in microorganisms varies and depends on species, strain and environmental and metabolic factors and can only be used as an approximation of the amount of bacteria in a solution (United States Environmental Protection Agency (US EPA) 2005; Hammes et al. 2010). To identify microbial contamination, it is advised that baseline conditions be established and monitored regularly. Any fluctuations from the baseline may indicate a change of microbial concentration and be an indication of contamination US EPA 2005). In an effort to simplify the conversion from ATP to number of cells, several conversions have been suggested. Comparing the number of cells counted during flow cytometry to the results of the ATP assay, Hammes et al. (2010) it was determined that the average amount of ATP per cell was $8.9 \times 10^{-17} \mathrm{~g} /$ cell. Deininger and Lee (2001) determined that the average amount of ATP per cell was approximately 1 fg (or $\left.10^{-15} \mathrm{~g} / \mathrm{cell}\right)$.

With the current state of knowledge, performance of the ATP assay varies with the selected assay as well as applied modifications to test procedures. Due to limitation in the detection limit of the test, assays are suitable to determine the microbial contamination or drift. With improvements, the ATP assay has potential to be more widely used as a water monitoring tool. 
To improve the performance of the assay, simpler and less time-consuming concentrations methods are needed. Improvements to the detection limits of current luminometers may also be possible. In addition, studies examine here have used real water samples that may contain mixed microbial fauna in a variety of states. The quantity of ATP/cell as well as the relationship with culture methods was variable. To better understand the relationship between ATP and the number of cells, it would be useful to examine the quantity of ATP of a single bacteria population in a controlled environment at different and known physiological states. While the ATP assay has been shown to be suitable for use with chlorinated water, a full investigation of the use of the assay to determine disinfection performance has yet to be completed. A study which examines a variety of chlorine concentrations is needed. For UV inactivation, there has only been one small study performed by Cho and Yoon (2007). A UV inactivation study is required that examines the full spectrum of UV irradiation doses (from 0 to $100 \mathrm{~mW} / \mathrm{cm}^{2}$ ) with particular attention being paid to UV doses that are employed for water and wastewater disinfection applications. 


\subsubsection{Available Technology}

Quench-Gone ${ }^{\mathrm{TM}}$ by Lumin Ultra Technologies (Fredericton, New Brunswick) www. Iuminultra.con

Quench-Gone $^{\mathrm{TM}}$ is a series of ATP assays produced by Lumin Ultra Technologies to measure the total microorganism population. Each test is specifically designed for the water, wastewater, industrial, pharmaceutical, and the food and beverage industries. A complete test, including sample preparation time can be completed within 5 minutes.

To complete a test, a water sample is first pushed through a filter attached to a syringe to capture bacteria on the filter paper. Then, a lysing agent is added to release the ATP from microorganisms. Finally, the solution is diluted, a luminescent agent is added, and the luminosity of the sample is measured by a luminometer. The luminometer is calibrated using the UltraCheck1 solution. The light readings can then be converted to active organism counts.

\section{ATPlite $^{\text {TM }}$ Luminescence Assay by Perkin Elmer (Woodbridge, Ontario) www perkinelmer.ca}

Perking Elmer provides a luciferase-based ATP luminescent assay that uses bioluminescent technology called ATPlites Luminescence Assay.

To complete the test, two reagents are added to a solution of lysed cells. The mixture is placed in the small wells of a microplate and gently mixed. The light produced is measured with a luminometer. This particular ATP assay allows for the light emission half-life to be extended to over 5 hours depending on the type of cell that is used. Results are generated within 15 and 25 minutes. Sensitivity of the assay depends on the type of cells used but as little as 5 cells were detected in $100 \mu \mathrm{L}$ of mixture using Chinese 
Hamster Ovary $(\mathrm{CHO})$ cells. The test was also shown to remain linear with cell concentration up to $10^{5}$.

\section{Profile-1® by New Horizons Diagnostic (Columbia, Maryland, USA)} www.nhdiag.com

Profile- $1 \circledR$ is ATP makes use of a Filtravette to determine the presence of bacterial ATP in a water sample. The Filtravette, manufactured by New Horizons Diagnostic, is a combination of a filter and a cuvette. It removes the free ATP from the solution and retains bacterial ATP on the filter paper. The Filtravette is then placed in a microluminometer and a lysing agent is added releasing the bacterial ATP. The released bacterial ATP is then mixed with luciferin-luciferase and the light emission is recorded (Schreppel et al. 2002). It is estimated that the Profile-1® system is able to detect ATP of as little as 200 cells (Schreppel et al. 2002).

\subsubsection{FLOW CYTOMETRY}

Flow cytometry measures scatter and absorption patterns of particles (including cells) as they flow through a light beam. The extent and nature of scattering and absorption are intrinsic properties of microorganisms, which can be correlated to the size, shape and density of microorganisms present in the sample (OECD and WHO 2003; Schreppel et al. 2002).

Fluorescent tags that bind to specific cell components such as DNA, RNA, antigens or other target molecules can be added to samples. This allows for the determination of total bacteria population, of selected types of bacteria and the viability of the bacteria. Flow cytometers are able to detect up to six parameters at one time and on one particle. Flow 
cytometry is very rapid with results provided in a matter of minutes (OECD and WHO 2003; Schreppel et al. 2002).

Flow cytometry was originally used to identify bacteria in hospital samples as well as in the food and beverage industry. In water monitoring, flow cytometry is used to identify Cryptosporidium parvum oocysts and Giardia cycsts using fluorescent labelled antibodies that bind specifically to these microorganisms (Ferrari et al. 1999). This method has been shown to be very precise and can produce results much faster than traditional plate counting (Chesnot et al. 2002).

Flow cytometry and fluorescence-based molecular identification has also been used to identify sub-bacterial population in activated sludge (Forster et al. 2003). The rapid detection of microbial species and their associated activities could enhance the treatment of wastewater.

\subsubsection{Available Technology}

FACSAria $^{\mathrm{TM}}$, FACSCalibur ${ }^{\mathrm{TM}}$ and FACSCanto ${ }^{\mathrm{TM}}$ by BD Bioscience Canada (Mississauga, Ontario)

www.bdbioscionces.ca

BD Bioscience is an international medical technology company with offices in over 50 countries. BD Bioscience Canada has three bench-top flow cytometers for purchase: the BD FACSAria $^{\mathrm{TM}}$, the BD FacsCalibur ${ }^{\mathrm{TM}}$, and the BD FACSCanto ${ }^{\mathrm{TM}}$.

\section{GEOMET Technologies Inc. (Maryland, USA)}

Www.geomer.com

In collaboration with the US EPA, GEOMET Technologies has developed a portable flow cytometer specifically designed for the rapid detection of adenovirus in wastewater. 


\section{Micro Pro ${ }^{\text {TM }}$ by Advanced Analytical Technologies Inc (Ames Iowa, USA)}

www.ati-us.com

Miro Pro ${ }^{\text {TM }}$ is a fully automated optical flow cytometer that can process up to 42 samples at one time. Samples are loaded in the instrument and the system automatically adds the reagent, mixes, and injects the sample. Both quantitative and qualitative results can be obtained with direct enumeration results available within 4 minutes and screening for microbial contamination within 18 to 24 hours.

Reagents used allow for labelling and fluorescent markers to be attached to specific compounds. Cells are then passed through a red diode laser and two photomultiplier tubes collect emitted signals from fluorescence and scatter. The system can detect microbial cells as small as $0.1 \mu \mathrm{m}$ using as little as $0.1 \mathrm{~mL}$ of a sample. The dynamic range of the system is of $10^{1}$ to $10^{6} \mathrm{CFU} / \mathrm{mL}$. Test kits are available to provide total viable organisms, total biomass or total dead cell counts.

The system has been evaluated by Schreppel (2003) to identify E.coli O157:H7 and Cryptosporidium. In both cases, microorganism were first separated from water samples using immunomagnetic separation and then labelled with specific fluorescent antibodies. The entire process allowed for identification of microorganisms in less than four hours. The Micro Pro ${ }^{\mathrm{TM}}$ flow cytometer is currently in use by the Mohawk Valley Water Authority (NY, USA) as part of an online monitoring system (Schreppel et al. 2002). 


\section{FlowCAM@ by Fluid Imaging Technologies (Yarmouth, Maine, USA)}

www fluidimaging.com

FlowCAM ${ }^{\circledR}$ automatically counts and characterizes cells of a continuous water flow in real-time and can also be used for a discrete sample. A digital image of each cell in a flowing steam of water is captured and associated data is presented in a spreadsheet. Each image is able to be stored with up to 26 different measurements such as length, width, area, and aspect ratio. FlowCAM® has been used to detect microorganisms (such as zebra mussel veligers and cyanobacteria), as well as to measure yeast growth during fermentation processes. It has also been used to identify protein agglomerates during the production of pharmaceuticals and to characterize the size distribution of particles (such as silicone gels and superabrasives).

By selecting images of a particle of interest, FlowCAM®'s software allows the user to build image libraries and create filters to remove or keep similar particles within a data set. With minimal sample preparation, dyes and buffers can be used to select particles that have been dyed. The instrument can also be set to only take pictures of molecules above or below a certain size. Depending on the selected magnification, FlowCAMß can detect particles ranging between $3 \mu \mathrm{m}$ and $3 \mathrm{~mm}$. The flow of water is constricted to match the selected level of magnification. 


\section{Microcyte ${ }^{\circledR}$ Aqua and Microcyte ${ }^{\circledR}$ Field by BioDetect (Houston, Texas, USA)}

www biodetect, biz

Microcyte $\mathbb{B}$ instruments use flow cytometry to quantify algae and other microorganisms.

The instruments are able to differentiate between biological and non-biological particles, require little sample preparation, and provide results in minutes. Microcyte $($ Field is a suitcase-sized portable flow cytometer where data can be presented as a two-colour histogram or dot plots directly on the instrument or on a PC. Microcyte $\AA$ Aqua is a stationary device designed for use during routine analysis along the treatment process. This instrument is suited for an online, continuous water surveillance system.

Both instruments are equipped with a laser diode that has a wavelength of $635 \mathrm{~nm}$ and uses fluorescence and light scattering to count microorganisms and particles in the water. The detection limit of the instruments depend on the background levels of the sample, however, a fluorescent particle, such as algae, may be detected to levels below 10 cells per $\mathrm{mL}$. The instruments can detect particles in the range of Particles between 0.4 to 15 $\mu \mathrm{m}$. Calibration, staining and maintenance kits that include buffer solutions are available from BioDetect. 


\subsubsection{Particle Counters}

Particle counters are typically used to determine the cleanliness and quality of air and fluids. Using light scattering, light blocking principles or microimaging, a small amount of flowing fluid is passed by the instruments detector. The instrument is then able to determine the quantity and size of particles in the flowing stream and establish the particle size distribution.

\subsubsection{Available Technology}

Micro-Flow Imaging instrument by Brightwell Technologies (Ottawa, ON) www.brightwelltech.com

Micro-Flow imaging instrument acts as particle counter and takes digital images of the particles in a flowing water sample. The instrument is able to record approximately one image per second and a one-millilitre sample of water takes approximately five minutes to analyze. The camera can capture the image of particles as small as $0.2 \mu \mathrm{m}$. Size and concentration of particles is analyzed and presented graphically. While the instrument can be operated continuously, it is designed to be used in a laboratory setting.

\section{Innosol-PLF by RandD Innovative Solutions Inc. (Ottawa, Ontario, Canada)} www.waterbiosafety.com

RandD Innovative Solutions Inc is a research and development company based in Ottawa Ontario Canada. Using a patent pending method, the Innosol-PLF instrument is able to detect, size and quantify biological and inorganic particulates of a large volume of water. The instrument is also able to collect and concentrate a water sample for further analysis without damaging the structure of the suspended solids and can be operated unattended with data being remotely downloadable. 
The instrument was able to detect $90 \%$ of bacteria introduced into a 1,000-Liter tank. Other capabilities of the device include detection of biological contaminants attached to inorganic particles and user defined measurement intervals. It is also self-calibrating, selfcleaning and results are available within 10 minutes.

During a recent demonstration study at the Britannia Water Purification Plant in Ottawa Ontario, the Innosol-PLF was used to maximize the use of inorganic and organic coagulants. During water treatment, the amount and ratio of organic and inorganic coagulants needed depend on the amount and ratio of organic and inorganic suspended solids are in the water. By dynamically measuring organic and inorganic suspended solids in the water, the chemical dose can be changed accordingly. Predicted cost savings is $30 \%$ of the current chemical cost.

\subsubsection{LIGHT SCATTERING TECHNOLOGY}

Particles scatter light when passed through a beam of light. Photo diodes collect the scattered light and translate it into a usable signal. The amount of scatter created by particles in water is called turbidity and is caused by microscopic organisms as well as organic and inorganic suspended matter. Highly turbid waters can encourage bacterial growth or limit the effect of disinfection. Turbidity meters detect particles within a certain size range but give no information on the nature of the particle. Thus, a microbe and a suspended grain of sand of similar sizes are considered equal. For this reason, turbidity meters are plagued with false positives and low levels of sensitivity. As part of a monitoring program, the Guidelines for Drinking Water Quality requires turbidity to be monitored on-line at intervals no less than five minutes to assure that filters are functioning properly (Health Canada 2006). 
Certain particles can scatter light in a particular way. New light scattering technologies are using multiple collection angles and algorithms to reduce the amount of false positives in a sample by recognizing that different particles can scatter light differently. Thus, water samples can be scanned to determine the size and number of a particular light scatting pattern. This allows for the distinction between a grain of sand and a microbe of similar sizes.

\subsubsection{Available Technology}

BioSentry $^{\text {TM }}$ by JMAR Technologies, Inc. (San Diago, California, USA) www.jmar.com

BioSentry ${ }^{\mathrm{TM}}$ is designed to be used as an EWS for the detection of pathogen contaminants in water. The system uses bio-optical signatures created by multi-angle light scattering (MALS) to distinguish between different organisms such as Giardia, E.coli and Cryptosporidium. It is also able to identify different states of the Cryptosporidium oocysts, including the type of treatment that was performed (ozone, heat, etc.) (Adams and Mccarty 2007).

BioSentry $^{\mathrm{TM}}$ is fully automated, able to be controlled remotely and allows for continuous, real-time monitoring of water distribution systems. Data is also available online. The limit of detection of the system depends on the user defined sampling intervals. A water of poor quality will have a greater number of false positives, due to interfering particles. The limits of detection are expected to decrease if the quality of the water decreases. When tested with filtered water, the system was able to detect less than one bacterium per 100 $\mathrm{mL}$ in four hours and 22 bacteria in $100 \mathrm{~mL}$ within five minutes (Adams and Mccarty 2007). 


\subsubsection{BIOSENSORS}

A biosensor is an analytical device that uses a "biological or biologically derived sensing element (e.g. enzymes, antibodies, microorganisms, or DNA)" (Dennison and Turner 1995) to measure the increased or decreased response in presence of a contaminant. A biosensor can use a physical (e.g. temperature), a chemical (e.g. pH) or a combination of physical and chemical signal, which can be interpreted by a transducer. In turn, a transducer can use an optical, electrochemical, or piezoelectric signal (Dennison and Turner 1995; Hart et al. 1993). Biosensors have the advantage of having a low cost, are portable, are easy to use, can provide a continuous signal, and, most importantly, can operate in situ (Dennison and Turner 1995). However, biosensors are not compatible with chlorinated waters and thus unsuitable to be used in water distribution systems (EPA 2005).

There are typically two categories of biosensors, those that (1) obtain a signal from a catalyst such as an enzyme or a microorganism, and those that (2) obtain a signal from some form of irreversible binding of a target molecule such as affinity sensors on antibodies or nucleic acids. The first type of sensor is most often seen.

Biosensors are widely used in wastewater application to determine the BOD of a sample. The typical structure of a microbial BOD sensor includes an oxygen probe and immobilized microorganism (Bourgeois, Burgess, and Stuetz 2001). The BOD measurement is based on the consumption of dissolved oxygen by the immobilized bacteria. Lehmann et al. (1999) developed a biosensor using a salt tolerant yeast microbe, Aruxla adeninivorans, to accurately measure BOD from wastewater in coastal and island or other high salt wastewaters. Pang et al. (2007) employed the luminescence the 
Stenotrophomonas maltophilia bacteria. The bacteria were loaded on a modified silica oxygen sensing film and luminescence measurements were done with a microplate reader. Results were obtained within 20 minutes (Pang et al. 2007).

Improving on this, Sakaguchi et al. (2007) immobilized the Photobacterium phosphoreum bacteria on a small acrylic chip measuring $3 \mathrm{~cm} \mathrm{X} 3 \mathrm{~cm}$. Luminescence was converted to BOD using a commercial digital camera and a laptop computer (Sakaguchi et al. 2007). Results were obtained within 20 minutes and the use of a chip allows for a high throughput and onsite detection of BOD. The system has a lower limit of $16 \mathrm{ppm}$ (Sakaguchi et al. 2007).

Immobilization of bacteria is not without its disadvantages. As the immobilization membranes age, measurements begin to "drift" and the DO probes have short lifetimes and require careful maintenance (Vaiopoulou et al. 2005). Membrane fouling of a biosensor is problem and frequent maintenance has also been reported (Chang 2003). To eliminate the membranes, Vaiopoulou immobilized bacteria on glass and measured the respiration activity $\left(\mathrm{CO}_{2}\right.$ concentration) of both immobilized and fluidized bacteria. This method produced continuous $\mathrm{BOD}$ measurement in real-time. In addition, the $\mathrm{CO}_{2}$ analyzer produced a digital signal making data available online.

Microbial Fuel Cells (MFC) use bacteria to degrade organic matter and to produce electricity. The electron transfer from the interior to exterior of the cell can be facilitated by chemical mediators or transferred directly to a metal electrode (Chang et al. 2003). A mediator-less MFC has been used as a BOD sensor in wastewater. A linear relationship was found up to BOD values of $100 \mathrm{mg} / 1$ and it was also shown that bacteria were able to 
recover after starvation periods and produced a background level current of $0.08 \mathrm{~mA}$ during starvation periods (Chang et al. 2003). Similar the previous example, the use of MFC allows for a continuous and real-time measurement of BOD without the use of problem causing immobilization membranes.

Non immobilized bacteria can also be used as biosensors as an assay where a luminescence response is measured by a portable luminometer. Bhattacharyya et al. (2005) tested five bacterial responses to pollution as a complement to an existing chemical analysis at a contaminated groundwater remediation site. In a remediation project, the biosensors are used to determine if remediation efforts are effective. The luminescent bacteria used included the metabolic bacteria, Vibrio ficheri, Pseudomonas fluorescens 10568, and Escherichia coli HB101, as well as catabolic bacteria E.coli DH5 $\alpha$, and Pseudomonas putida TVA8 (Bhattacharyya et al. 2005). The catabolic biosensors detect toluene and middle-chain alkanes respectfully. Catabolic biosensors estimate the bio-availability of the contaminant they detect. The metabolic biosensors are used to appraise the potential of bioremediation (Bhattacharyya et al. 2005).

As previously mentioned, biosensors convert a biological response into a detectable signal. As such, biosensor are not only applicable to BOD measurements in wastewater but have also been applied to water applications to detect: phenolic compounds, bisphenol A, heavy metals, organic phosphates, polycyclic aromatic hydrocarbons, pesticides and herbicides (Farré et al. 2009). Rodriguez et al. (2002) used a biosensor derived from the algae C. vulgaris to detect herbicides in water. Algae are present in waters exposed to light. It has been shown that fluorescence patterns of chloroplasts of algae change when exposed to herbicides. Fluorescence induction curves produced by $C$. vulgaris were used 
to detect chemicals such as methyl parathion, cyanide, paraquat dichloride (herbicide) in surface waters. Algae, immobilized on a sensor, was able provide continuous in situ measurements in a simulated river flow (Rodriguez, Sanders, and Greenbaum 2002).

\subsubsection{SENSOR-ARRAY TECHNOLOGY}

Sensor-array technology or "electronic noses" have been used to detect headspace odours in air. Electronic noses are able to characterize an odour based on its chemical composition (Bonastre et al. 2005). Carleton University (Ottawa, Ontario) is currently working with the Canadian Food Inspection Agency (CFIA) to develop a sensor-array that could identify bacteria in the food industry.

Most sensors have a sensitivity and selectivity for a narrow number of parameters. Sensor array technology typically use less selective sensor that is sensitive to a number of components in the water. Then, through case-base reasoning solutions a current problem is matched with a solution to similar a past problem (Colilla, Fernandez, and Ruiz-Hitzky 2002). Colilla et al. (2002) have used this technique to distinguish between samples of mineral waters containing mixtures of different ions. The future could see the application of sensor array technology for water quality monitoring. 


\subsubsection{OTHER AVALABLE TECHNOLOGY}

\section{The Biolog System by Biolog (California, USA)}

www biolog.com

Biolog System is a biochemical identification kit used to identify unknown bacteria. The test is comprised of a 96 well microplate where each well contains a different selection of carbon sources, dried nutrients and biochemicals. An isolate of an unknown bacteria inoculated on either a gram-positive or a gram-negative microplate. To make the isolate, the unknown bacteria must first be isolated and grown on an agar media. After a period of incubation, between 4 to 6 and/or 12 to 24 hours, respiration of the bacteria has utilized the carbon sources of selected wells to produce a purple colour in those wells. The pattern created by the purple wells is then cross-referenced with a library through computer software, which is able to identify approximately 339 gram-positive and 526 gramnegative species of bacteria. (States et al. 2002)

\subsubsection{OTHER EMERGING TECHNOLOGY}

\subsubsection{Quantum Labelling}

Quantum labelling is the use of nanocrystals that fluoresce at a variety of wavelengths to identify sub-cellular components of living cells. Also known as quantum dots, there are three layers to the structure of this material. The core, made of semiconductor material, is encased with a shell designed to improve the optical properties of the sphere. In turn, polymers coat the shell and bind to biological material. The use of this material is similar to that of other labelling methods. The quantum dots can attach to other molecules, such as target DNA sequences, antibodies, or receptors and instruments will measure the level of light emitted. However, unlike other methods that use fluorescent markers such as 
PCR, the use of quantum dots does not require amplification steps and biological molecules can be quantitatively determined (Hasan et al. 2005; Lee et al. 2004)

Currently on the market, Qdots ${ }^{\circledR}$ are nanocrystal spheres developed by the Quantum Dot Company located in Hayward, California, USA (www.qdots.com) for the use of quantum labelling. Recently, the use of Qdots has been used for the enumeration of C. parvum oocysts. The US EPA method 1622 prescribes the use of organic fluorophores such as fluorescein isothiocyanate (FITC) for the identification of transmissive stage C. parvum oocysts. However, the organic fluorophores used for labelling are prone to photo degradation, which shortens the time at which measurements are accurate. Qdots have a higher resistance to photo degradation. A method developed by Lee et al. enumerated $C$. parvum successfully with Qdots by labelling an antibody of C. parvum (Lee et al. 2004).

The US EPA Office of Ground Water and Drinking Water (OFWDW) Technical Support Center is currently developing a portable sensor using quantum dots to identify cyanobacteria and cyano toxins in water.

\subsubsection{Magnetic Beads}

Dynal Biotech, Norway (www.mvitrogen.com/site/us/en/home/brands/Dynal.hml) has developed microscopic beads that are superparamagnetic; they are only magnetic when exposed to a magnetic field. The beads, measuring between 1 and $4.5 \mu \mathrm{m}$ are coated with a polymer. To this polymer, antibodies, proteins, RNA/DNA probes, etc. can be attached and, in turn, combined with target molecules such as selected pathogens. The probe-target complexes can be easily separated from the liquid by applying a magnetic field. 


\subsection{Monitoring Chemical Contaminants}

Effects of microbiological contaminants are often acute and, as a result, prescribed regulations require frequent monitoring to assure public safety. On the other hand, effects of chemical contaminants are typically chronic in nature or are all together unknown. Changes in water chemistry tend to be long term unless a specific contamination event has occurred. Regulatory testing requirements are thus less frequent or altogether nonexistent (Gheewala et al. 2003).

The urban water matrix is a complex mixture of very low concentrations of chemical compounds. These compounds appear in water because they are continuously used in industry, agriculture and by urban populations, and they have limited removal rates from conventional water treatment trains. Compounds that persist in the environment can accumulate to concentrations that can affect human health. Recent technological developments and associated research activities have allowed for detection of contaminants that would previously go undetected. Research activity has also focused on the effects of continuously released low concentration contaminants. Increased awareness of the effect of persistent molecules will increase monitoring requirements (Gheewala et al. 2003).

There are a series of difficulties that arise when trying to develop monitoring techniques. To begin with, extraction and concentrating procedures are typically required. These procedures are detailed, require expert personnel and are inherently difficult to automate. Real-time chemical monitoring requires probes to be in constant contact with water or wastewater. This is problematic because instruments are prone to fouling which results in loss in sensitivity and reproducibility. 
It can also be difficult determine the exact chemical associated with a specific symptom or series of symptoms. However, with development of new detecting methods and recent research efforts, new and emerging chemical contaminants are raising awareness of the importance of testing for low concentration chemical contaminants. There is a future need for real-time monitoring of chemical contaminants to assure the long-term public health (Bonnastre et al. 2005).

Currently, there is a large amount of research activity being performed to developed new detection methods and techniques for emerging contaminants of concern. However, few have been made commercially available thus far.

\subsubsection{CHEMICAL CONTAMINANTS OF CONCERN}

\subsubsection{Arsenic}

Arsenic is a heavy metal naturally found in the earth's crust. It is odourless, tasteless and most known for its use as a poison. Recently, contaminated drinking water wells have caused arsenic poisoning in some parts of the world, notably in Bangladesh and India (Katsoyiannis and Zouboulis, 2006).

Chronic exposure to arsenic in drinking water can cause skin lesions and can lead to cancer. In Canada, the drinking water guideline is set at $0.010 \mathrm{mg} / \mathrm{L}(10 \mu \mathrm{g} / \mathrm{L})$ and treatment devices are available for the removal of arsenic (Health Canada, 2006).

The analytical method most commonly used to determine the arsenic concentration in solution is gaseous hydride formation (GHAA). This method measures characteristic wavelengths produced from the formation of arsenic hydrides under certain conditions. Spectroscopy-based methods are also common and include inductively coupled plasma 
mass spectroscopy (ICP-MS), selective ion monitoring with ICP-MS, and inductively coupled plasma atomic emission spectroscopy (ICP-AES). These methods of detection require a high level of skill and high capital cost to purchase the equipment (Health Canada, 2006).

\subsubsection{Cyanobacteria Toxins}

Cyanobacteria, or blue-green algae, produce a number of neurotoxins, hepatotoxins, skin irritants and other toxins that can contaminate drinking water and cause severe acute reactions. In surface water, most commonly different forms of the hepatotoxin microcystin are found and can cause illness through skin contact or ingestion. Canadian guideline recommends a microcystin-LR level below $1.5 \mu \mathrm{g} / \mathrm{L}$ though it is recommended to monitor the amount of total microcystins (Health Canada, 2006).

Time and duration of toxin algae blooms depends on environmental conditions and can vary from year to year. Conditions such as an increased temperature, turbidity or nutrient levels are favourable to the formation of blooms. Toxins can persist in the environment of up to 21 days.

While the level of toxins associated with illness has not been established, microcystins can cause stomach cramps, vomiting, diarrhoea, fever, headaches, muscle and joint pain and weakness as well as gastroenteritis, immune function and liver problems. Microcystins can cause acute and chronic health effects. 
There are few standard analytical techniques to assess microcystins in water but research in this area is ongoing. Some of the developed screening methods include mouse bioassay, ELISA, and phosphatase bioassay. Liquid chromatography coupled with UV adsorption detection (HPLC-UVD) or mass spectrometry (MS) methods are also available. However, these methods require sample preparation and extraction procedures are needed to separate the different microcystins in water and a number of tests are used to detect each different type of microcystins (Health Canada, 2006; Mountfort et al. 2005).

Conventional treatment that includes coagulation, clarification and filtration is effective at removing cyanobacterial cells. However, increasing sludge removal and filter backwash rates from clarification and filtrations processes respectively is recommended. The use of any chemical that can cause cell lysis should be avoided. This would cause toxins to be released into water. Removing toxins from water is more difficult but certain oxidation procedures and activated charcoal have been found to be effective (Health Canada, 2006).

\subsubsection{Pesticides/Herbicides/Insecticides and Degradation Products}

Pesticides are a group of chemicals used to deter or kill pest found on agricultural fields. This group of chemicals may also include herbicides and insecticides. In order to be effective, pesticides are designed to be resistant to environmental factors such as rain and UV radiation. However, it is these same characteristics that allow pesticides to persist and accumulate in the environment. The effect of pesticides on humans can be vast but severe. For example organophosphates and carbonates tend to behave like nerve agents, by inhibiting the enzyme cholinesterase, which allows muscles to relax after a contraction (Richardson and Ternes, 2005). 
Recently, there is more concern awarded to pesticide metabolites (also referred to as degradation products) than to parent pesticide molecules. This is because degradation products can often be found at greater levels than the parent pesticide. Two chemicals of a particular concern are acetanilide pesticide degradation products, and triazines and their degradation products. Degradation products are typically more polar than their parent molecules and thus more difficult to detect. Combinations of liquid chromatography (LC) and mass spectrometry (MS) are often used. (Richardson and Ternes, 2005).

\subsubsection{EMERGING CONTAMINANTS}

Emerging contaminants of concern are chemicals that have previously gone undetected because of analytical limitations, of a lack of associations with environmental or health effects or they have just recently been introduced to the environment. Emerging contaminants are unregulated because, until recently, there has not been a cause for concern and the long-term effects of these contaminants are still unknown (Poynton and Vulpe, 2009).

The challenge with emerging contaminants is that they represent a wide range of chemicals that have very unique characteristics. Chemicals that are of a particular concern are those that are highly persistent and are bioavailable to be taken up by organisms (Poynton and Vulpe, 2009). Examples of recent emerging contaminants of concern include polybrominated diphenyl ether (PBDE) flame retardants, sunccreen/UV filters, contaminant disinfection by-products, pesticides and degradation products and chemical warfare agents (Richardson and Ternes, 2005). 


\subsubsection{Pharmaceuticals and Personal Care Products}

Pharmaceutical and personal care products (PPCP) are a group of chemicals that are continuously released through personal care activities in low concentrations. These include compounds needed to make painkillers, antibiotics, contraceptives, antidepressants, sunscreens, lotions to name a few. However, due to the wide use of PPCP, their cumulative concentration is significant. Human and livestock excretion are the primary pathways of PPCP into the environment. Discarding expired or unused products can also be a significant pathway.

Pharmaceuticals are of a particular concern because they contain active ingredients and are designed to be bioavailable. In addition, while acute toxicity information is typically required and readily available, the chronic long-term effects of exposure are not well studied (Poynton and Vulpe, 2009). Of particular concern is the accumulation of antibiotics in water sources and the study of antibiotics is a major area of research in the field of veterinary studies. The concern is that accumulation of antibiotics in the environment will create resistant strains of bacteria (Richardson and Ternes, 2005).

PPCP represent a large group of contaminants with a wide range of characteristics. There is thus not one analytical method able to identify all PPCP. There are over 3,000 different compounds used for the formulation of PPCP however, only approximately 150 of these have developed detection methods. In addition, PPCP are present in very low concentrations. This requires complex concentration, extraction and clean up procedures before identification methods can be applied (Kullman et al. 2009; Richardson and Ternes, 2005). 


\subsubsection{Endocrine Disrupting Chemicals}

Endocrine disrupting chemicals (EDC) are a group of natural and synthetic hormones typically in found in pharmaceutical products as well as pesticides, plasticizers and organic metal compounds. This group of hormones may have a variety of chemical characteristics but are unique in the fact that they all affect the normal function of the endocrine system; a system of glands that regulate the release of hormones. Disrupting the endocrine system may have effects on reproduction, development and immune functions of living organisms. Typical detection methods include a combination and variations of methods that include liquid chromatography (LC) and mass spectrometry (MS) (Poynton and Vulpe, 2009).

\subsubsection{Brominated Flame Retardants}

Polybrominated diphenyl ethers or PBDE are a group of brominated compounds used as flame-retardants for polymers and plastics. PBDEs are of a concern because they are very stable compounds and thus persist in the environment. Brominated flame-retardants are known neurotoxin in mammals and are able to bio-accumulate in living organisms (Poynton and Vulpe, 2009).

\subsubsection{Fluorinated Compounds}

Fluorinated compounds are used widely industrially for their unique chemical properties. They are hydrophobic, lipophilic and moderately soluble. Unfortunately, this group of chemicals is very stable and extremely persistent in the environment (Poynton and Vulpe, 2009). 


\subsubsection{Nano-Materials}

Nano compounds are very small particles, ranging from $0.1 \mathrm{~nm}$ to $100 \mathrm{~nm}$ in diameter.

The characteristics of the particle are as varied as the applications for which they are designed. The effect of each particle must be assessed separately. The use of nanomaterials is expected to grow exponentially in the next ten years (Poynton and Vulpe, 2009).

\subsubsection{COLORIMETRIC METHODS}

Colorimetric methods are portable colour reaction kits typically used for rapid onsite analysis. To complete a test, a water sample is mixed with a series of reagents. A colour will appear if a certain contaminant is present.

Colorimetric methods are available for a wide variety of contaminants, are easy to use and easy to transport. The concentration of the contaminant is determined by comparing colour gradient produced to that of standard gradients. Visual or electronic readings are also possible. Quantitative results are thus also possible with calorimetric methods (EPA 2005).

To determine the amount of arsenic in water, a calorimetric method that uses the Gutzeit method is often applied. This method reduces soluble forms of arsenic to arsine gas with the addition of zinc in acidic conditions. The arsine gas $\left(\mathrm{AsH}_{3}\right)$ is the then trapped in a silver diethyldithiocarbamate solution or with mercury bromide. Comparing the colour produced from the series of reactions to a standardized colour chart allows for quantification of arsenic in water. Arsine gas is toxic and work should be carried out in a well-ventilated space. It should be noted that the Gutzeit method has difficulties 
quantifying small quantities of arsine and production of hydrogen sulphide may interfere with the results (Dhar et al. 2004).

\subsubsection{Available Technologies}

Arsenic Quick ${ }^{\mathrm{TM}}$ by Industrial Test Systems, Inc. (South Carolina, USA) www.sensafe.com

Arsenic Quick ${ }^{\mathrm{TM}}$ test kits utilize a series of three reactants and an arsenic test strip. Reactants are added to a water sample in a specific order and allowed appropriate amounts of time to react. A test strip is then inserted into the bottle and the colour produced on the strip is compared to a colour chart to determine the concentration. During the reactions, inorganic arsenic $\left(\mathrm{As}^{+3}\right.$ and $\left.\mathrm{As}^{+5}\right)$ is converted to arsine $\left(\mathrm{AsH}_{3}\right)$ using ferrous and nickel salts as catalysts. When arsine is applied to the mercuric bromide test strips, a colour change from white to yellow to brown is observed. The test has a detection limit that ranges between 0 to $0.5 \mathrm{mg} / \mathrm{L}$. Using a dilution of one over five, concentrations up to $2.5 \mathrm{mg} / \mathrm{L}$ can be detected. Results are obtained within 15 minutes.

\section{AS 75 by Peters Engineering (Austria)}

\section{Peters.engineeringastyria.com}

AS 75 is a field-portable colorimetric test to determine the arsenic concentration of a sample. Indicator tablets are placed into a water sample. Intensity of the colour produced, if any, is compared visually to a colour gradient chart. A battery-operated tester can also be used. 


\subsubsection{IMMUNOASSAYS}

Immunoassays are tests that measure the response of the combination of an antibody and its associated antigen. The immune system triggers the creation of an antibody, a protein, when there is an invading antigen in the body. Immunoassays test for the presence of the antigen or the antibody. Depending on the test, a label is applied to either the antibody or antigen and produces a response once combined. Radioactive, luminescent or an enzyme that changes colour is often used.

Immunoassays have high sensitivity and specificity, are rapid and cost-effective. They are also easy to use; require small volume and no pretreatment. In addition, simultaneous measurement of a number of samples is possible and tests are available to screen for a number of different contaminants. Immunoassays are useful to screen for a group of contaminants where further testing is required for positive test.

Two popular immunoassays are the enzyme-linked immunosorbent assay (ELISA) and the immuno affinity chromatography (IAC). IAC is particularly suitable for polar substances (Gheewala et al. 2003).

Research is ongoing to develop immunoassays for new substances because they are rapid, inexpensive and easy to use. For example, a fully automated optical immunoassays was recently developed. River Analyser (RIANA) uses total internal reflection fluorescence (TIRF) to detect very low levels of contaminants. Fluorescently tagged antibodies are given time to react with a water sample before being streamed through a flow cell. A laser is directed at the flow cell and a signal created by the antibody-antigen complexes sends a signal through polymer fibres to photo diodes that are connected a computer. The entire 
process requires about 12 minutes to complete with a limit of detection as low as 0.20 ng/L for estrone. The system should also able to detect pharmaceuticals, antibiotics, hormones endocrine disrupting chemicals and pesticides in water (Tschmelak et al. 2005).

There are over 60 different types of congeners of microcystin toxins produced by cyanobacteria and an ELISA is useful to determine the total amount microcystins in a water sample. The ELISA uses an antibody to detect a unique amino acid called ADDA found in the most microcystins toxins. However, the toxicity of a type microcystins can vary. The results of the protein phosphatate test will vary depending on the cogeners present and their level of toxicity. Recently, Mountfort et al. (2005) have used an ELISA in conjunction with a phospatase inhibition assay called PP-2A to measure the toxicity of a mixture. The authors developed an index called a response ratio which is created by dividing the amount determined by PP-2A test by the total amount determined by the ELISA. A high ratio indicates a highly toxic mixture. While the PP-2A is just as rapid as and ELISA, there are no field portable versions and the test must be performed in the lab (Mountfort et al. 2005). 


\subsubsection{ANODIC STRIPPING VOLTAMMETRY}

Anodic stripping voltametry (ASV) measures the current induced in an electrochemical cell. This method is used to determine the amount of metal ions in solution. Mercury and/or arsenic ions are able to be detected by ASV.

The working principle of ASV is simple. A potential applied to a solution will reduce metal ions of concern to its metal form, once the electrode has reached the ionizing potential of the metal ion. Then, the metal form of the ion accumulates on the surface of the electrode. Once a sufficient amount of metal has accumulated, the potential is increased at a constant rate to oxidize the metal. The oxidizing potential is also specific to the metal concentration of concern. The current produced by the oxidation is measured and plotted as a function of the potential applied. To determine the original concentration of the metal ion in solution, a calibration curve is compared to the area under the curve of the tested solution (EPA 2005).

\subsubsection{Available Technologies}

\section{PDV6000plus by Cogent Environmental (UK)}

www.cogentenvironmental.co.uk

PDV6000plus is a portable device that uses ASV technology to measure the concentration of ions in solid mixtures and in solutions. Before measurements are taken, a 30-minute calibration procedure is required. Then, approximately five to seven minutes are needed to complete the measurements. 


\section{SafeGuard $^{\mathrm{TM}}$ II and ArsenicGuard ${ }^{\mathrm{TM}}$ by TraceDetect (Seattle, Washington, USA)}

www rracedetect.com

TraceDetect designs a number of trace metal analyzers with a focus on "close to realtime" procedures. The ArsenicGuard ${ }^{\mathbf{T M}}$ is an automated online arsenic analyzer able to monitor up to four sample streams with one system. The system will automatically grab a sample and perform the analysis. Results are obtained within 30 minutes and the system has a detection limit of $1 \mu \mathrm{g} / \mathrm{L}$.

SafeGuard ${ }^{\mathbf{T}}$ II is designed to automatically analyze grab samples with the push of a button. The system can be configured for arsenic, copper, cadmium, lead, zinc or mercury. The detection limit for all analytes is $1 \mu \mathrm{g} / \mathrm{L}$ and results are obtained within 30 minutes. The SafeGuard ${ }^{\mathrm{TM}}$ can be placed at-line or in the quality assurance lab.

Both systems use patented ASV technology called Nano-Band ${ }^{\mathrm{TM}}$. The three electrodes of the Nano-Band ${ }^{\mathrm{TM}}$, the explorer electrode, the auxiliary electrode and the reference electrode, are encased together for ease of operation and replacement.

\subsubsection{GAS CHROMATOGRAPHY (GC)}

Gas chromatography is a laboratory procedure used for the identification of organic chemicals such as industrial chemicals and components of fuel oils. Compounds are vaporized, mixed with a carrier gas and transported through a packed column, which is heated in an oven. During vaporization, individual compounds are separated from each other. The time that each compound takes to reach the end of the column depends on the characteristics of the compound and the carrier gas. GC is often combined with thermal conductivity detector (TCD), surface acoustic wave (SAW), electrolytic conductivity 
detector (ECD), electron capture detector, flame ionization detector (FID), photo ionization detector (PID) and mass spectrometer (Gheewala et al. 2003).

Analysis using GC can be performed grab samples a number of times per day or performed automatically at regular intervals. However, the use of GC devices requires skilled operators and regular maintenance. The use of GC may also be too expensive to be run on a continuous basis. GC is typically laborious, time consuming, involves extensive pretreatment and expensive laboratory equipment (EPA 2005).

Portable GC-MS devices are now available for the use in field measurements. Compared to laboratory testing, field measurements offer quicker results and reduced risk for contamination. This allows for rapid determination contaminants such as chemical weapons or other toxic pollutant (Casson et al. 2006).

\subsubsection{Available Technology}

Scentograph CMS500 and Scentograph CMS200 and HAPSITE ${ }^{\circledR}$ by INFICON (New York, USA)

www.inficon.com

Scentograph CMS500 is an automated gas chromatograph used for the online determination of volatile organic compounds (VOCs) such as trihalomethanes (THM). The system requires no sample pretreatment or filtration steps. The system also has very low detection limits reaching parts per million and trillion levels. The Scentograph CMS500 provides real-time measurements and has the capacity to program sampling intervals. The Scentograph SMS200 is a portable version of the online system. Results from both systems are obtained within minutes (EPA 2005). 
HAPSITE is a portable GS-MS system used to measure hydrocarbons in air, soil and water samples. The gas chromatographer is able to detect VOCs with molecular weights ranging between 45 and $300 \mathrm{~g} /$ mole. The mass spectrometer uses electron multiplier detector and has a library of 170,000 organic compounds. The HAPSITE system is also equipped with an in situ probe that automatically collects and concentrates samples (Casson et al. 2006).

\subsubsection{LIQUID-CHROMATOGRAPHY (LC)}

In recent years, liquid-chromatography (LC) coupled to mass spectrometry (MS) has replaced the GC analytical methods for the detection of emerging contaminants. Compared to GC, LC-MS requires reduced sample pretreatment and enhanced selectivity. LC-MS is suitable to determine small concentrations of pharmaceuticals, hormones and endocrine disrupting compounds. Additionally, tandem analysis is now possible and increasingly popular. However, pre-concentration of the sample is still required. LC-MS also suffers from matrix effects such as ion suppression and it can be difficult to separate highly polar substances. Recent research has focused on the development of new method to remedy this problem. Solutions include development of 13C-labelled internal standards to reduce the effects of the matrix and the use of ion-pair reagents or hydrophilic interaction liquid chromatography (HILIC) for the analysis of polar compounds. (Rodriguez-Mozaz et al. 2007; Richardson and Ternes, 2005).

A variety of LC techniques have been applied for the detection of cyanobacteria toxins. While LC is typically coupled with MC including tandem mass spectrometric techniques (MS/MS), methods such as UV absorption at fixed frequencies, selected ion monitoring (SIM) can also be used (Maizels and Budde, 2004). However, a well optimized method 
will be selective and sensitive with low limits of detection and quantification (LOD and LOQ, respectfully) to produce results in a reasonable amount of time.

Toxic and non-toxic strains of cyanobacterial species have little predictable differences, thus further physiochemical, biochemical or biological methods are required. Liquid chromatography-electrospray ionization mass spectrometry (LC-ESI-MS) was used to screen for toxic forms of cyanobacteria toxins such as major types of microcystins and nodularins. This method provided a high throughput of samples with a total analysis time of 2.8 minutes. A detection limit of 50-100 pg/injection was achieved. However, this time does not include sample pre-treatment's that would be required if dealing with a field sample (Meriluoto et al. 2004).

Cyanobacteria toxins of a particular concern include neurotoxins anatoxina-a and cylindrospermopsin and cyclic heptapeptide microcystin hepatotoxins such as microcystin-LA, microcystin-RR and microcystin-YR. The latter group was separated in less than 30 minutes using reverse-phased liquid chromatography columns and a time of flight mass spectroscopy (LC/TOF-MS). The TOF-MS allows for the acquisition of full spectrum data and real-time exact $\mathrm{m} / \mathrm{z}$ measurements. In this case, toxin were eluted from the water using extraction disks containing very fine $\mathrm{C}-18$ silica particles in a Teflon matrix (Maizels and Budde, 2004).

Another examples uses liquid chromatography hyphenated with electro-spray ionization triple quadrupole mass spectrometry (LC-ESI-MS/MS) was used to detect cyanobacterial toxins microcystins and nodularin in both lake water and drinking water. The method was able to optimize the mobile-composition of the MS. This method used a reversed-phase 
guard cartridge system as the analytical column thus eliminating the need for sample preconcentration procedures, such as SPE, and allowing for a short detection time of 10 minutes. The achieved limit of detection is $0.27 \mathrm{ug} / \mathrm{L}$ and limit of quantification is $0.9 \mathrm{ug} / \mathrm{L}$ which are both well below the Canadian guideline limit of 1.5ug/L (Allis et al. 2007).

\subsubsection{MICROCHIP TECHNOLOGY}

Microchip technology is the miniaturization of immobilized assays on chips that can be used as sensors. Recently advancements have seen the development of a new class of microchips call "lab-on-chips". This class of microchips are able perform analysis that previously needed the use of a laboratory to complete. The miniaturization of bench-top equipment allows for quicker and portable analytical processes.

Microchip technology also allows for a number of different analyses to be carried out simultaneously as different methods can be imprinted side-by-side on a same chip. Biochips are a type of microchip that relies on biological reactions such as nucleic acid hybridization, antibody reaction, and enzymatic reactions (EPA 2005).

Microchip technology can miniaturize a number of different types of analytical techniques. For example, Yuan and Carmichael (2004) developed a microchip to identify cyanobacterial toxins microcystin and nodularin. The microchip used surface-enhanced laser desorption ionization (SELDI) mass spectrometry to complete the capturing, purification, analysis and processing of complex biological mixture. The chip was used to identify microcystins and nodularins in flood and liver tissue sample with a detection limit of $2.5 \mathrm{pg}$ of microcystin-LR detected in $2 \mu \mathrm{g}$ of water (or $1.2 \mu \mathrm{g} / \mathrm{L}$ ) (Yuan and Carmichael, 2004). 


\subsubsection{DNA MICROARRAY}

As described in Section 2.4.4 for the detection of biological contaminants, DNA microarray is a sequence of genes immobilized on a solid surface in a particular order. The hybridization between the immobilized gene probes and a target gene produces a signal that can be detected. (Brousseau, 2005; Dorigo et al. 2005).

A DNA microarray can also be used to detect chemical contaminants in water. For example, significant concentrations of a pharmaceutical substance can have an effect on the gene molecules of different species. Using the transformed gene, a probe can be created and immobilized on a surface. The detection of the transformed gene would indicate that the chemical necessary to create the change is present in the water. The bioassay would thus asses the change induced by the chemical and not the chemical directly. The challenge is to find a gene transformation that is specific to a particular chemical (Kullman et al. 2009).

Though successful proof of concept studies are currently being performed, there are a number of obstacles that must be overcome before wide use of DNA microarrays is possible. The next research steps might include establish geographical and demographic pharmaceutical patterns, determine non-overlapping gene markers, determine dose and time response effects and determine the effects of the gene markers in a typical water matrix. In addition, as described in Section 2.4.4, the use of DNA microarrays requires DNA to first be extracted. This pre-processing step is lengthy, time consuming and limits the real-time use of DNA microarray (Kullman et al. 2009). 


\subsubsection{SOLID PHASE EXTRACTION}

Solid Phase Extraction (SPE) is laboratory procedure often employed to concentrate water samples for subsequent analysis, such as LC-MS. SPE can be used to extract semivolatile or non-volatile analytes of a liquid solution. Two techniques are typically employed. The analyte or everything else can be held on a solid surface, or the stationary phase. Then the analyte is either washed from the surface of the stationary phase or eluted from the liquid phase. The choice of stationary phase and solvent are thus very important and depend on the chemical and physical properties of the analyte.

Compared to other sample extraction and concentrating procedures, SPE is advantageous because it can be automated and depending on the choice of stationary phase, can be very selective. However, it is limited to pre-selected chemicals and must be used in conjunction with subsequent laboratory techniques in order to analyses any one characteristic of water quality (Richardson and Ternes, 2005).

With new emerging contaminants of concern, new stationary phases and solvents are continually in development. Generic sorbents are commonly based on hydrophobic interactions and the most popular include alkyl-bonded silicas, copolymer sorbents and hydrophilic lipophilic balanced polymers. Recent more specific designed sorbents have used molecularly imprinted synthetic polymers (MIPs) or immobilized receptors or antibodies (immunosorbents) and restricted access materials (RAMs) (Richardson and Ternes, 2005). 
On-line SPE units are now also available. These units allow for the reduction of sample preparation time and an increase of sample throughput, decrease risk of contamination and not degradation or loss of the analyte. Online SPE units perform analysis on the entire sample, thus also leading to lower limits of detection. Application of an on-line SPE-LCMS has been used, in the water industry, for the detection of pesticides, phenols and polycyclic aromatic hydrocarbons (PAH), caffeine, disinfection by-products and endocrine disrupting chemicals (Richardson, 2009).

\subsubsection{Available Technology}

\section{PROSPEKT by Spark Holland (Netherlands)}

www.sparkholland.com

PROSPEKT is a commonly used SPE unit. This system is a programmable online SPE. The Prospek-2 decreases the time needed to produce results by working in parallel with two separate samples.

\section{GX-271 ASPEC ${ }^{\mathrm{TM}}$ and GX-274 ASPEC ${ }^{\mathrm{TM}}$ by Gilson (Wisconsin, USA). Www gilson com}

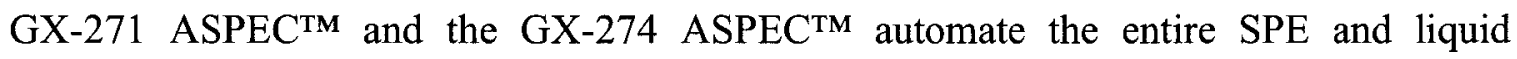
handling processes; from initial conditioning steps to the final transfer. The instruments are also equipped with positive pressure valves to ensure positive flow rates which in turn ensure less contamination between samples. With these instruments, the SPE process can be completed in approximately 10 minutes and the instruments can be operated in both batch and sequential modes. The GX-247 is able to process up to four samples in parallel. 


\subsubsection{OTHER EMERGING TECHNOLOGY: FIBRE OPTICS}

A research group at the Great Lakes Water Institute, University of Wisconsin is developing a real-time water distribution monitoring system that uses fibre optics. Receptors and a variety of fluorescent groups coat the outside of a fibre optic cable which is then run through a water distribution pipe. If a toxin binds to a receptor, then the fluorescent group emits light. The fluorescent groups are specific to different contaminants thus knowing where the light is being emitted will allow for the determination of the type of contaminant present (EPA 2005).

Similarly, the Intelligent Optical Systems Inc located in California, USA (www.intopsys.com) has a product called DICAST $®$. Permeable indicators coat the entire length of fibre optic cables giving them sensitivity to a chemical. This system is currently designed for the use in air pollution but can potentially be modified for water monitoring purposes (EPA 2005).

\subsection{MONITORING TOXICITY}

Considering the large variety of contaminants, online analytical methods are costly and impractical as each contaminant would be separately tested. Rapid toxicity testing assesses the general safety of water. An ideal toxicity assay would require little time, skill and money to complete and detect the presence of toxic substances at concentrations above what would cause a public health risk. Test should also be quantitative and reproducible. Toxic substances to consider include heavy metals, industrial chemicals, chemical warfare agents and biotoxins. A positive result indicates a need for more testing and a negative result would not necessarily assure water safety. If tests are completed in 
real-time, a grab sample taken the moment a negative test was obtained would be useful to perform further analysis (Casson et al. 2006).

\subsubsection{AVAILABLE TECHNOLOGY}

\section{LuminoTox by Lab_Bell Inc. (Shawinigan, Québec, Canada) www lab-bell.com}

Lumino Tox assay uses plant and algae derived enzyme extracts. These extracts consist of photosynthetic enzymatic complexes (PECs) that emit fluorescence when exposed to light. An analytical instrument designed by Lab_Bell is able to measure the inhibition fluorescence in the presence of toxic substances such as herbicides, organic solvents, ammonia nitrogen and organic amines. Sensitivity of the test is increased if incubation is increased.

Online version of the LuminoTox test called Robot LuminoTox is also available. This instrument is self-cleaning, is able to provide measurements every 30 minutes and store data in an excel file.

\section{Eclox Rapid Toxicity System by Servern Trent Services (England) www. severntrentservices.com}

Eclox Rapid Toxicity System was developed by the UK armed forces to evaluate drinking water quality in the field, evaluate raw water, develop a treatment plan and provide quality control of output water from treatment.

Eclox is a chemiluminescent test that provides a quantitative assessment of water quality. The test relies on the reaction between luminol and an oxidant in the presence of horseradish peroxidase (HRP), a catalyst. This reaction produces a flash of light that is measured with a luminometers. If pollutants are in the water, then the reaction is inhibited 
and the light production is less. Free radicals, antioxitants as well as phenols, amines and heavy metals all interfere with the reaction. To complete the test, $1 \mathrm{~mL}$ of water is added to reagents and placed in the luminometer for four minutes. Results are compared to a control of deionized water. The luminometer can hold up to 60 measurements which can be downloaded to a computer.

\section{Rapid Field Enzyme Test by Servern Trent Services (England) www.severntrentservices.com}

Rapid Field Enzyme Test uses the inhibition of the enzyme cholinesterase to detect nerve agents and pesticides. A membrane disk saturated with cholinesterase is dipped into a water sample for one minute and pressed against another disk containing an ester for three minutes. If no pollutants are present, the enzyme is not inhibited and the disk turns blue. If pollutants are present the disk remains white. Results are obtained within 5 minutes. The detection ranges for the pesticides carbamates, thiophosphates and organophosphates were 0.1 to $5 \mathrm{mg} / \mathrm{L}, 0.5$ to $5 \mathrm{mg} / \mathrm{L}$ and 1 to $5 \mathrm{mg} / \mathrm{L}$ respectfully.

\subsubsection{USE OF ORGANISMS AS SENSORS}

Toxicity testing of a water supply can be done with living organisms such as bacteria, water fleas, mussels and fish where the physiological or behavioural changes are most often monitored (Hansan et al. 2005). Also referred to as biosensors, living organisms provide a rapid response to a wide range of contaminants. Typically, the response of a living organism is monitored and compared to a baseline response. If a threshold is exceeded, an alarm is triggered (Mikol, et al. 2007). Responses can also be monitored in real-time to provide continuous toxicity screening. 
Biosensors are typically unable to determine the concentrations of individual contaminants. To maintain the health of an organism, an appropriate amount of care must also be given. Pathogens are species specific and thus biological organisms are typically not able to detect human pathogens (Mikol, et al. 2007; Gerhardt et al. 2006).

The choice of a biological organism is specific to the type of response required. A suitable organism must be sensitive to chemicals at low enough concentrations that it will prove useful, have a known response to toxicity and have a response that is long enough to be able to be detected. It is also important to develop a response plan to confirm positive events and rule out false alarms. The use of cells is increasingly popular because the latter can be modified to contain a specific response, such as bioluminescence, and are able to be miniaturized (Hansan et al. 2005; Mikol, et al. 2007).

\subsubsection{MICROORGANISMS AS SENSORS}

Bacteria can rapidly detect toxins in water. Disruption of cell metabolism is typically monitored by a reduction in bioluminescence. Cell growth patterns by means of turbidity or cell density is also useful indication of toxicity (Gerhardt et al. 2006). Kits of freezedried bacteria are widely available and convenient to use and test results are available within 30 minutes. Bacteria are able to detect pesticides, herbicides, chlorinated hydrocarbons and heavy metals. Problems encountered when using bacteria biomonitors include clogging, continuous growth of the bacteria and the need to add nutrients and media (Gerhardt et al. 2006; Hasan et al. 2005).

In addition to respiration rate and bioluminescence, more recently developed biosensor are based on such measuring principles as the transformation of carbon, sulphur and nitrogen, the activity of enzymes and glucose uptake (Gheewala et al. 2003). 


\subsubsection{Available Technologies}

\section{MICROTOX® and DeltaTox® by Strategic Diagnostic (Newark, Delaware, USA)}

www.sdix.com

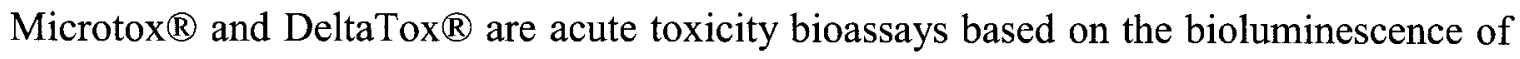
Vibrio fischeri bacteria. Chemical energy produced by metabolic activity of bacteria is converted into visible light. A change in cell respiration results in a measurable change in luminescence. The change in luminescence is compared to a control sample and software is able to identify the toxic substance. Results can be obtained in less than an hour and multiple tests can be run simultaneously. Test bacteria are freeze dried and do not require any special care.

Mirotox ${ }^{\circledR}$ is for use in the laboratory while DeltaTox ${ }^{\circledR}$ is a portable field version. Equipped with a self-calibrating photometer, a photo-multiplier tube and data collection software, results are promptly available within five and 15 minutes. Both instruments are sensitive to chlorine and thus not suitable for use in the distribution system. 
LUMI CELL® ER bioassay by Xinobiotic Detection Systems, Inc. (North Carolina, USA)

Www.dioxins.com

LUMI CELL® ER bioassay uses a human ovarian carcinoma cell that contains an estrogen-responsive luciferase reporter gene (BG1Luc4E2) which emits light in response to estrogenic compounds. Estrogenic compounds can have an effect on the endocrine and reproductive systems of human and other animal species (Xinobiotic Detection Systems, 2005). This test is only available off line because replacement of the organisms is needed after each measurement.

\section{ToxScreen by CheckLight Ltd (Israel)}

\section{checklight.biz}

ToxScreen developed by CheckLight Ltd uses luminescent bacteria Photobacterium leiognathis to detect $\mu \mathrm{g} / \mathrm{L}$ concentrations of toxic organic and inorganic pollutants. This bioassay is easy to use and is able to detect heavy metals, pesticides, polycyclic aromatic hydrocarbons (PAHs) and chlorinated hydrocarbons within 20 to 45 minutes. Light emitted from the bacteria changes in response to chemophysical and biological toxicant that affect cell respiration and rate of protein or lipid synthesis. Each toxicant is distinguished by differences in the response of the bacteria. The test is thus able to determine the toxicity of a water sample with the use of a simple luminometers. Replacement of a buffered solution and fresh freeze-dried bacteria are required every 14 days. 


\subsubsection{ALGAE AS A SENSOR}

Algae are typically used to detect herbicides in water through measurements of cell growth, oxygen production, chlorophyll fluorescence, or the mobility of the flagellate (Gerhardt et al. 2006). Measurements taken from algae have been performed on a semicontinuous basis with measurements taken in intervals of 30 minutes. Algal suspensions are typically used though standardization procedures are difficult (Gerhardt et al. 2006; Gheewala et al. 2003; Hasan et al. 2005).

\subsubsection{Available Technology}

\section{Algae Toximeter by bbe moldaenke (Germany)} www bbe-moldaenke.de

Algae toximeter places algae in a regulated fermenter where the activity of the algae is monitored. Water samples are injected into the instrument and changes in algae's levels of fluorescence are monitored. If the algae's activity is constant, then no toxic substances are present in the water. This test is not suitable for use in chlorinated water.

\subsubsection{BIVALVES AS A SENSOR}

Behavioural patterns of bivalves, mainly shell closure, can also be monitored. Two commercial monitoring systems that employ bivalves include the Dreissena-Monitor (Germany) and Musselmonitor ${ }^{\circledR}$ (Delta Consult, The Netherlands). 


\subsubsection{Available technology}

\section{Musselmonitor(B) by Delta Consult (Netherlands)}

www mosselmonitor.n1

Musselmonitor ${ }^{\circledR}$ correlates valve position of mussels, Dreissena polymorpha, to temperature, concentration of suspended material (turbidity and chlorophyll concentration) and general toxicity of the water. The system was developed by studying the normal behaviour of mussels considering seasonal variations (Sluyts, et al. 1996). The behaviour of eight mussels is continuously monitored for up to two months providing a continuous supply of nutrients is supplied. Software enables graphical representation of the individual behaviour of each mussel. Musselmonitor $®$ is use by Waterworks of Budapest, Hungary.

\subsubsection{DAPHNIA AS A SENSOR}

The first toxicity test to employ a living organism recorded dynamic movements of the water flea or Daphnia as it passes through an infrared light beam. With advancement of image recognition equipment, video-based technology is being developed to monitor swimming behaviour of individual water fleas to provide real-time results. To maintain a healthy population of water fleas, some level of skill is required. Water fleas require a continuously algae feed. (Gerhardt et al. 2006; Gheewala et al. 2003). 


\subsubsection{Available technology}

IQ-Tox TM by Aqua Survey, Inc, (New Jersey, USA)

Www.kingwooddiagnostics.com

IQ-Tox ${ }^{\mathrm{TM}}$ toxicity test utilizes Daphnia magna to detect chemicals and biological toxins. In the presence of a toxin, the metabolism of the Daphnia magna is reduced which can be detected through fluorescent tagging. Results are available within 75 minutes and these organisms are sensitive to chlorine residual.

In 2003, the US EPA Environmental Technology Verification program found that amongst other toxicity tests evaluated; the IQ-Tox test identified the most amounts of chemicals below the human lethal dose concentrations. A summary of the findings is presented in Table 2.6.

Table 2.6: Summary of US EPA ETV Results

\begin{tabular}{|l|l|l|}
\hline Contaminant & $\begin{array}{c}\text { Human Lethal Dose Concentration } \\
(\mathbf{m g} / \mathbf{L})\end{array}$ & $\begin{array}{l}\text { Toxicity Threshold } \\
(\mathbf{m g} / \mathbf{L})\end{array}$ \\
\hline Aldicarb & 280 & 3.5 \\
\hline Colcicine & 240 & 24 \\
\hline Cyanide & 250 & 0.25 \\
\hline Dicrotophos & 1,400 & 0.88 \\
\hline Thalium & 2,400 & 125 \\
\hline Botulinum Toxin & 0.30 & 0.0003 \\
\hline Ricin & 15 & 0.015 \\
\hline Soman & 0.3 & 0.0013 \\
\hline
\end{tabular}




\section{Daphnia Toximeter by bbe moldaenke (Germany)}

www.bbe-moldaenke.de

Daphnia Toximeter allows for swimming speed, altitude, number of turns, circling movements, growth rates and number of live daphnia to be used as a toxicity screening method. Data is available online and measurements are performed in 30 minutes intervals.

\section{Multispecies Freshwater Biomonitor by LimCo International (Germany) www. limco-int.com}

Multispecies Freshwater Biomonitor $(B)$ monitors the behaviour response of up to 96 different micro-vertebrates and is able to collect readings every ten minutes (Gerhardt and Schmidt, 2002, 2006). Each specimen is placed in a chamber where the instrument records movements and ventilation patters. Software analyses the collected signal which is then correlated to toxicity of the water. The MFB can be used for both sediment and water analysis (De Bisthoven, et al. 2004).

\subsubsection{FISH AS A SENSOR}

When fish are employed to monitor the toxicity of water, the rheotaxis of fish is typically visually monitored (Gerhardt et al. 2006). Rheotaxis is the tendency of fish to always face the current. If downstream movement is observed, then fish are trying to move to a more favourable water condition and toxicity can be inferred (Gerhardt et al. 2006). Other methods record ventilation rate, cough rate, heart rate, and other swimming behaviours

(Gerhardt et al. 2006). Fish are sensitive and can provide real-time response if standardization problems can be overcome. Fish cells are a possible alternative to the use of whole organisms however cells are typically not as sensitive (Gheewala et al. 2003). 
In particular, the bluegill fish (Lepomis mchrochirus) has been used and a total of four parameters can be measured: (1) ventilatory rate, (2) cough rate, (3) ventilatory depth and (4) movement of the each fish at 15 second intervals (Mikol et al. 2007; van der Scharlie et al. 2004). Basic water quality parameters such as temperature, dissolved oxygen, conductivity, and $\mathrm{pH}$ are also monitored. A toxicity index is created by combining baseline behavioural data with the basic water characteristics. Should the index exceed a certain threshold for more than $70 \%$ of the fish, then an alarm is triggered. Bluegills are especially sensitive to chemicals that cause direct gill damage such as zinc or residual chlorine (Mikol et al. 2007; van der Scharlie et al. 2004). Bluegill fish have been used successfully to detect toxic contaminant in Fort Detrick (USA) and by the New York City Department of Environmental Protection (USA) (Gerhardt et al. 2006).

A research group at the University of Wisconsin's Great Lakes Water Institute have created a biosensor for use in the distribution system using transgenic zebrafish. After fertilization, zebrafish embryos are injected with pollution-responsive reporter genes designed to detect 18 chemical contaminants. The presence of one of these contaminants in the water supply will trigger a response which will produce luciferase in the fish. Light emitted by the luciferase can then be detected. 


\subsubsection{Available Technology}

\section{Fish Bio-sensor ${ }^{\circledR}$ by Biological Monitoring Inc (Virginia, USA)}

www biomon.com

Fish Bio-Sensor ${ }^{\circledR}$ monitors the electric field of 8 to 12 fish. Any deviation from normal condition is indicative of a toxin. When this occurs, an alarm will sound and a water sample is collected to allow for further analysis. The system is equipped with an automatic feeder and a de-chlorination module can be added. The Fish-Bio-sensor has been used in Singapore, Australia and South Africa.

\subsection{SUMMARY OF FINDINGS}

This chapter aimed to look at new and innovative strategies and methods to more quickly evaluate water quality parameters. Modern water quality issues are complex and municipalities must deal with aging infrastructure and increasing public awareness. With more of Canada's population living in urban centers than ever before, even short-term water quality issues have the potential to affect a large number of people.

Constant monitoring and a quick response are vital to the protection of public health. The development of a tried and strategic approach to monitoring is important to screen for contamination and allow time to mediate the problem. The use of on-line real-time sensors that are already in place is practical and economical. The use of biological organisms as a type of sentry alarm system is also important though the use of microorganisms and cells may be more practical. In addition, light-scattering technology may also be a useful tool to determine general water quality parameters such as dissolved organic matter (DOM) and biochemical oxygen demand (BOD). While these technologies 
are limited in their ability to identify specific contaminants, they offer simplicity, rapid analysis and, most importantly, are low-cost.

New biological monitoring techniques aim to be quicker and more specific than before. Conventional and real-time PCR as well as DNA microarrays can identify single molecules but require the use of detailed pre-processing techniques and the use of specific laboratory equipment. For chemical contaminant monitoring, the current focus is to develop new detection methods for emerging contaminants of concern. For this, liquid chromatography is becoming increasing more efficient. The limiting factor for laboratory methods appears to be development of more efficient sample concentration techniques and may be a long time away from real-time analysis. For quick and easy field analysis, the use of immunoassays is a better choice.

The successful transition of an emerging technology onto the market place depends on the magnitude of the improvement it can offer, the ease of use an implementation, the reliability of the results but most importantly cost. For a municipality or other treatment facility to invest in a new technology is has to be worth the investment. 


\section{CHAPTER 3}

\section{RATionale FOR The SElection OF THE ATP ASSAY}

The purpose of the literature review was to identify new and innovative ways to monitor the quality of water and wastewater in real-time and to select one of the technologies for further evaluation. The ATP luminescence assay was selected for further examination for its potential to be a powerful monitoring and optimization tool for water and wastewater quality monitoring.

The following chapter will describe the working principles of chlorine disinfection (Section 3.1), UV irradiation (Section 3.2) and the ATP assay (Section 3.3). In addition, this chapter aims to make clear why the use of the ATP assay is advantageous and why it was selected for further testing.

\subsection{ChLORINE Disinfection}

Chlorine is the most widely used disinfection method that works by oxidizing cell membranes, attacking other organic elements of the cell and ultimately killing the microorganisms (Water Environment Federation (WEF) 1996).

Chlorine can be added to water as a gas or as a liquid in a number of different forms. For this study, a liquid solution of sodium hypochlorite (NaOCL) was used. Once added to water, sodium hypochlorite will first hydrolyze to hypochlorous acid, $\mathrm{HOCl}$, and sodium hydroxide, $\mathrm{NaOH}$, see Equation 3.1). The hypochlorous acid will then ionize to produce a 
hydrogen cation, $\mathrm{H}^{+}$, and a hypochlorite anion, $\mathrm{OCL}^{-}$, see Equation 3.2 (Metcalf \& Eddie 2003).

$$
\mathrm{NaOCl}+\mathrm{H}_{2} \mathrm{O} \rightarrow \mathrm{HOCl}+\mathrm{NaOH}
$$

$$
\begin{gathered}
\mathrm{HOCl} \leftrightarrow \mathrm{H}^{+}+\mathrm{OCl}^{-} \\
\text {where } K_{i}=\frac{\left[\mathrm{H}^{+}\right][\mathrm{OCl}]}{[\mathrm{HOCl}]}=3 \times 10^{-8} \frac{\text { moil }}{2} \text { at } 25^{\circ} \mathrm{C}
\end{gathered}
$$

The amount of chlorine available for disinfection is called free chlorine and is equal to the sum of the amounts of hypochlorous acid and hypochlorite ion present in the water. The proportion of each species is temperature and $\mathrm{pH}$ dependant. In addition, hydrochlorous acid is between 40 and 80 times more effective as a disinfectant than hypochlorite. (Metcalf \& Eddie 2003). At ambient temperature and neutral pH, the proportion of $\mathrm{HOCl}$ is approximately $75 \%$ (Metcalf \& Eddie 2003). Generally, the acidity of the hypochlorous acid will react with the alkalinity in the water sample, to maintain the $\mathrm{pH}$ near neutrality. Chlorine disinfection is measured by the $\mathrm{CT}$ value, which is the multiplication of the chlorine concentration $\mathrm{C}\left(\mathrm{mg} / \mathrm{L} \mathrm{Cl}_{2}\right)$ and the contact time, $\mathrm{T}(\mathrm{min})$

To test the effectiveness of chlorine disinfection there are two strategies are jointly used. First, the free chlorine residual can be measured in real-time. The chlorine residual, or amount of hypochlorite, assures that enough chlorine has been added to the water to react with oxidizable substances and an excess if available. Measurement of the residual free chlorine is not a direct indication of the microbial quality of water. For this, lengthy culture methods are used to detect culturable cells. Culture methods may also under 
estimate the number of microbial cells because they do not account of the viable but nonculturable cells.

\subsection{UV INACTIVATION}

UV inactivation is the process in which UV light absorbed by cells damages the nucleic acid of cells to prevent replication. If a cell is unable to replicate, it is unable to cause infection. The two most common forms of nucleic acid in the cell are deoxyribonucleic acid DNA and ribonucleic acid RNA which absorb UV light between the wavelengths of 200 and $300 \mathrm{~nm}$, peaking at $260 \mathrm{~nm}$.

DNA and RNA are composed of pairs of purines and prymidines where each purine is paired with prymidine. When exposed to UV light, the most common mechanism of inactivation is the creation of prymidine dimmers, see Figure 3.1, where prymidines bond to each other instead of to their complementary purine. Damage to DNA and RNA caused by UV light inhibits the replication of cells while the cellular structure, and what it contains, remains intact (EPA 2006). 


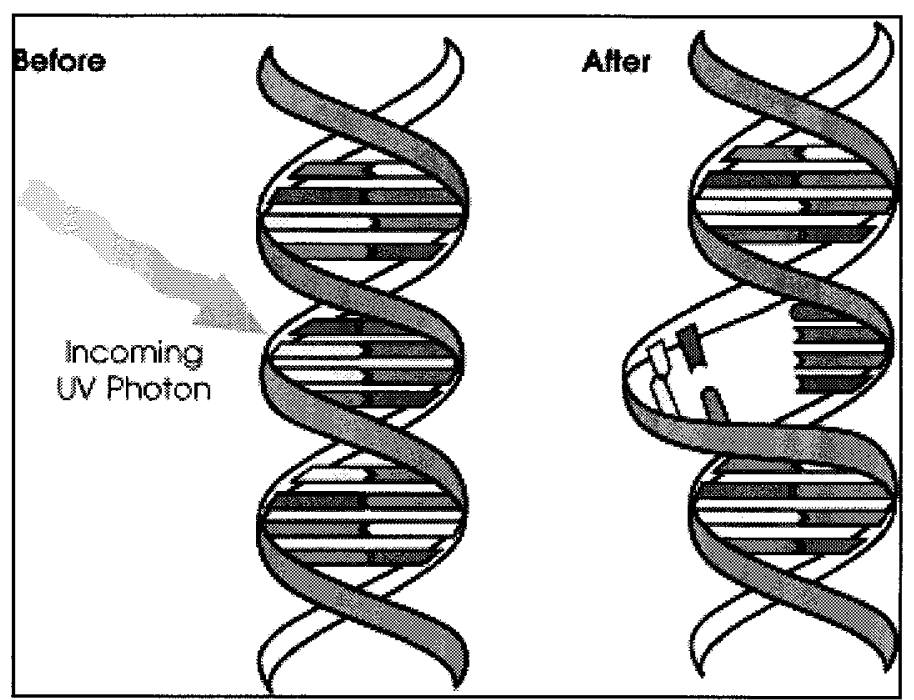

Figure 3.1: Illustration of effect of UV light on the structure of DNA. Before, each purine is paired with a prymidine across the double helix structure. After, formation of prymidine dimmers within a helix. Source (Allen 2001).

UV light is typically produced by low pressure mercury vapour lamps, which produce monochromatic light at a wavelength of $254 \mathrm{~nm}$. However medium pressure mercury vapour (polychromatic), xenon and excimer lamps are also available. The effectiveness of UV irradiation is measured by the UV dose $\left(\mathrm{mJ} / \mathrm{cm}^{2}\right)$ which is the product of the radiation intensity ( $\mathrm{mW} / \mathrm{cm}^{2}$ ) and contact time (s) (Bolton and Linden 2003).

Unlike chlorine disinfection, UV dose is a physical process and does not leave a chemical residual in water. Currently there is no quick evaluation method for UV disinfection performance. Culture plate methods and other time-consuming procedures are the only measure of the effectiveness of UV disinfection. It is suspected that ATP molecule has reduced activity after exposure to UV irradiation. If there is a significant reduction in ATP activity which can be measured with the ATP assay, then the ATP assay may be a novel real-time monitoring tool for UV disinfection of water. 


\subsection{The ATP Bioluminescence AsSAY}

The working principle ATP bioluminescent assays is a light producing reaction that involves the ATP molecule and two compounds extracted from fireflies: luciferin and luciferase. In theory, the light produced can be quantified and is proportional to the amount of ATP present in the sample. Light is produced when luciferin is oxydized to oxyluciferin by ATP in the presence of luciferase and magnisium ions $\left(\mathrm{Mg}^{2+}\right)$ as follows:

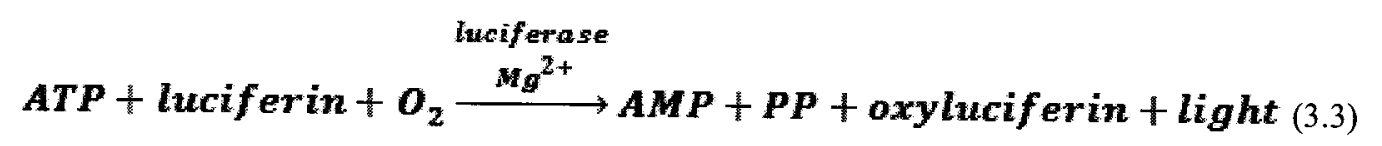

Source: (LuminUltra Technologies 2010a)

where adenosine triphosphate is represented by ATP, the oxygen molecule by $\mathrm{O}_{2}$, AMP is adenozine phosphate and PP is pyrophosphate (LuminUltra Technologies 2010; Shimomura 2006).

Luciferase is an enzyme that catalyzes the reaction, shown in Equaiton 3.3, by lowering the requred activation energy. Magnisium is an activaor which is also needed to complete the reaction (Rao 2006). In the absence of Luciferase and magnesium, the reaction would still occur but at a much lower rate. Enzymes are proteines that fold into three dimentional structues to create active sties that bind to the substrate, in this case luciferin. The "lock and key" mechnism holds the substrate in place with weak bonds until the reaction has completed. Luciferase, is specific to this reaction however it is possible that other molecules bind to the enzyme to reduce its activity or (Rao 2006). Luciferase has been shown to be inhibited by fatty acids, anesthetics (including alcohols and alkanes) and substrate-related, intermediate and product compounds (Denburg and McElroy 1970; Leitão and Esteves Da Silva 2010; Szaecka, Xu, and Tang 2007). The strongest inhibitors 
are the substrate-related, intermediate and product compounds of the reaction which act as a natural feedback mechanism. To counter this effect, enzyme solutions of ATP assays include substances that react with these compounds and stabilize the light production (Leitão and Esteves Da Silva 2010). Divalent cations have also been investigated as a competitive inhibitor to the magnesium ion $\left(\mathrm{Mg}^{2+}\right)$. Nickel $\left(\mathrm{Ni}^{2+}\right)$ and cobalt $\left(\mathrm{Co}^{2+}\right)$ ions were the strongest inhibitors however, chloride $\left(\mathrm{Cl}^{-}\right)$has also been shown to act as a noncompetitive inhibitor (Leitão and Esteves Da Silva 2010).

The ATP assay is simple, easy to use, can be completed in five minutes, but most importantly it can detect all viable cells (LuminUltra Technologies 2010a). Other molecular methods, such as the polymerase chain reaction, measure the amount of DNA of a sample, which can survive after the death of a cell. These methods measure the amount of viable and of non-viable cells (Bushon, Likirdopulos, and Brady 2009). The ATP molecule, on the other hand, is involved in the energy transfer of living cells. If a cell dies, the ATP molecule quickly decomposes and can no longer be detected (Bushon, Likirdopulos, and Brady 2009; Deininger and Lee 2001). Other current microbiological testing methods, such as culture plate methods, rely on cell replication to detect the presence of bacteria. However, if a cell is stressed or exposed to a new environment (i.e., $\mathrm{pH}$, temperature, nutrient constitution) it may be viable but not able to replicate and, in turn, not culturable (Berney, Weilenmann, and Egli 2006; Colwell 2000). In addition, plate count methods use specific agars to select for heterotrophic bacteria. While heterotrophic bacteria are associated with fecal contamination they do not represent the entire microbiological population (Deininger and Lee 2001). The plate methods give a 
partial representation of the water quality, while the ATP method measures the ATP from all microbial sources.

\subsubsection{THE QUENCH-GONE AQUEOUS (QGA) TEST KIT}

LuminUltra Technologies Inc (Fredericton, NB) has a variety of ATP assays for different applications (drinking water, wastewater, sludge etc.). In particular, the Quench-Gone Aqueous (QGA) test kit is reported to detect between 0.1 and $667,000 \mathrm{pg} \mathrm{ATP} / \mathrm{mL}$ which makes it suitable for finished drinking water and wastewater. In addition, this particular test kit has the ability to measure only cellular ATP and thus the complete population of viable microbial cells (LuminUltra Technologies 2010a).

Other ATP assay kits and procedures have been evaluated for the use with natural and treated water as well as raw and treated wastewater. Based on the results recent research, see Section 2.4 .5 , the sensitivity limit of a variety of ATP assay range between $10^{1}$ and $10^{2}$ cells per $\mathrm{mL}$ and depends on the assay selected as well as applied optimization procedures. However, ATP assays examined utilize a plate luminometer which limits sample volumes between 0.1 and $10 \mathrm{~mL}$. What is unique about the QGA kit is that it makes use of a syringe to collect cells from a sample on a filter paper, which are subsequently lysed and pushed through to be collected in a small vial. Sample volumes of $100 \mathrm{~mL}$ are recommended and larger sample volumes are possible.

To be a successful monitoring tool for UV irradiated samples, there must be a significant decrease in the quantities of ATP measured before and after disinfection. LuminUltra provided results from two preliminary field trials that tested surface water and wastewater effluent before and after UV disinfection with the ATP assay. The first trial measured the change in the quantity of ATP with time of a surface water sample subsequent to UV 
irradiation using both low and medium pressure UV lamps (Figure 3.2). The results of the UV irradiated samples with low pressure lamps, show that as time progressed the quantities of ATP gradually decreased from $65 \mathrm{pg} / \mathrm{mL}$ to $20 \mathrm{pg} / \mathrm{mL}$ (at 24 hours). These results would suggest that allowing an incubation time subsequent to UV irradiation would increase the difference in ATP and thus the effectiveness of the ATP assay. When medium pressure lamps were employed, there was an immediate decrease in the quantity of ATP from $65 \mathrm{pg} / \mathrm{mL}$ to less than $1 \mathrm{pg} / \mathrm{mL}$ rendering the incubation period unnecessary.

The second trial occurred at a wastewater treatment plant and the summary of the results can be seen in Table 3.1. The wastewater treatment plant typically reports a $2-\log$ reduction of bacteria after UV disinfection, although no plate counts were performed by LuminUltra to confirm this value (LuminUltra Technologies). The testing was done only at one UV dose, which was the operational UV dose $\left(60 \mathrm{~mJ} / \mathrm{cm}^{2}\right)$ of the treatment plant. The company reported a $14 \%$ reduction of ATP before and after UV disinfection with no pretreatment. Then, four pretreatment methods were developed which increased the percent reduction in ATP to a maximum of $98 \%$. The term pretreatment method refers to treatments applied to UV disinfected samples before the application of the ATP assays. The pretreatment methods aimed to increase the difference between the amounts of ATP measured before and after UV inactivation by providing a shock to the cells and quickening the decay of ATP activity after UV exposure. The results from the initial field trials indicated that the QGA ATP assay had promise as a monitoring tool for UV disinfection. ATP assay is also likely to be successful for monitoring chlorine disinfection since chlorine destroys cellular and extracellular ATP. ATP assay is also likely to be 
successful for monitoring the microbial contamination in water supplies as this would be directly related to the ATP content.

As previously mentioned, light is produced from a reaction between ATP and luciferin in the presence of luciferase and magnesium. LuminUltra's proprietary mixture of luciferin, luciferase and magnesium is called Luminase and initial testing was completed with this reagent (LuminUltra Technologies 2010a). However, during this study it became evident that there were problems with the sensitivity of the ATP test and the company provided XXL Luminase which included higher concentrations of luciferin and luciferase to increase the amount of light produced from the aforementioned reaction.

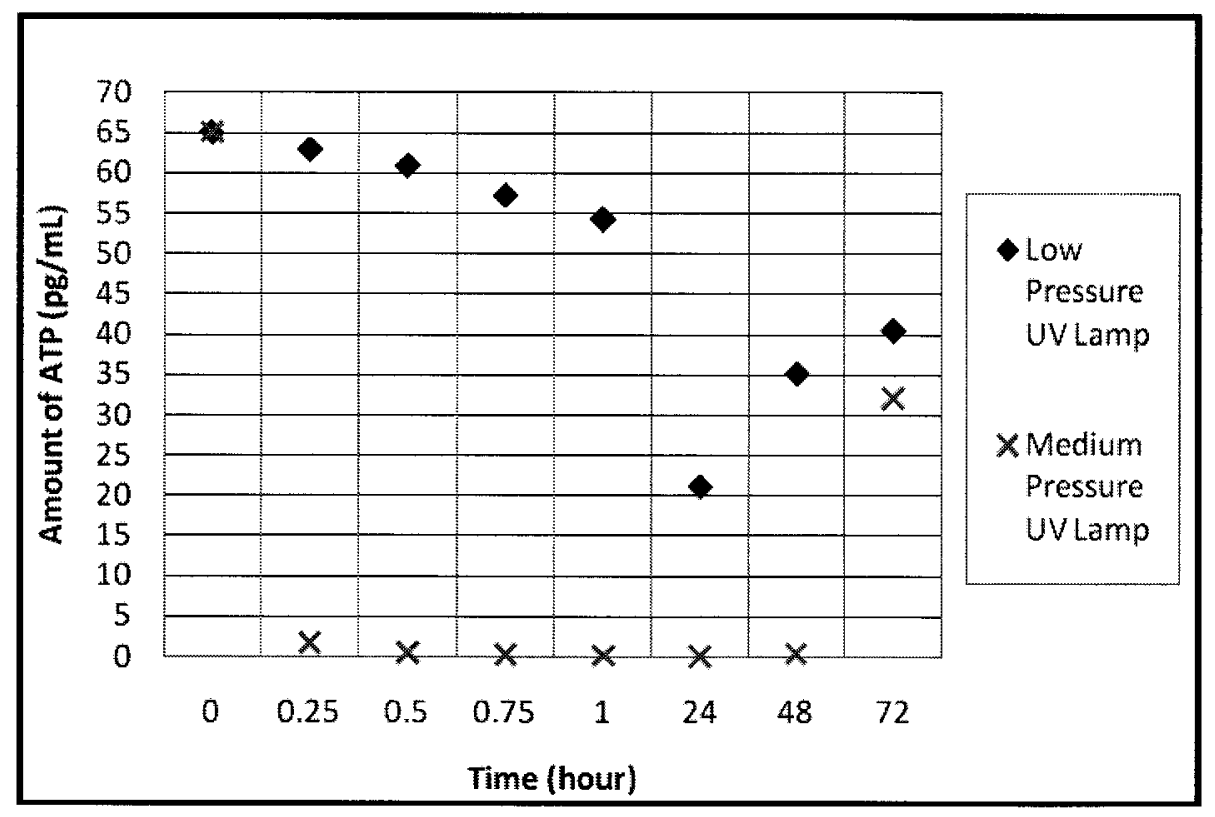

Figure 3.2: Change of the amount of ATP of a surface water sample with time subsequent to UV irradiation with low and medium pressure UV lamps. Source: (LuminUltra Technologies) 
Table A. 1: Effect of four pre-treatment's on the percent reduction of ATP of effluent wastewater samples before and after UV irradiation. Source: (LuminUltra Technologies)

\begin{tabular}{|c|c|}
\hline Pretreatment Applied Before ATP Analysis & $\begin{array}{l}\text { Percent ATP } \\
\text { Reduction* }\end{array}$ \\
\hline No Pretreatment & $14 \%$ \\
\hline $\begin{array}{l}\text { Pretreatment Method no. 1: } \\
\text { 1. Add } 1 \mathrm{~mL} \text { of LumiSolveX } 10 \text { to } 9 \mathrm{~mL} \text { sample } \\
\text { 2. Incubate for } 20-30 \text { minutes at ambient temperature } \\
\text { 3. Neutralize to } \mathrm{pH} 7.5 \\
\text { 4. Spike with yeast extract concentration to obtain a final } \\
\text { concentration of } 1000 \mathrm{ppm} \\
\text { 5. Incubate for } 30 \text { minutes } \\
\text { 6. Proceed with ATP assay procedures }\end{array}$ & $21 \%$ \\
\hline $\begin{array}{l}\text { Pretreatment Method no. } 2 \text { : } \\
\text { 1. Spike } 9 \mathrm{~mL} \text { of sample with yeast extract concentration to obtain a } \\
\text { final concentration of } 1000 \mathrm{ppm} \\
\text { 2. Incubate for } 30 \text { minutes } \\
\text { 3. Add } 1 \mathrm{~mL} \text { of Lumisolve } 10 \mathrm{X} \text { to sample } \\
\text { 4. Incubate for } 30 \text { minutes at ambient temperature } \\
\text { 5. Proceed with ATP assay procedures }\end{array}$ & $28 \%$ \\
\hline $\begin{array}{l}\text { Pretreatment Method no. } 3 \text { : } \\
\text { 1. Spike } 9 \mathrm{~mL} \text { of sample with yeast extract concentrate to obtain a } \\
\text { final concentration of } 1000 \mathrm{ppm} \\
\text { 2. Incubate for } 2 \text { hours } \\
\text { 3. Add } 1 \mathrm{~mL} \text { of LumiSolve10X to sample } \\
\text { 4. Incubate for } 20-30 \text { minutes at ambient temperature } \\
\text { 5. Proceed with ATP assay procedures }\end{array}$ & $37 \%$ \\
\hline $\begin{array}{l}\text { Pretreatment Method no. } 4 \text { : } \\
\text { 1. Add } 1 \mathrm{~mL} \text { LumiSolve } 10 \mathrm{X} \text { to } 9 \mathrm{~mL} \text { sample } \\
\text { 2. Incubate for } 30 \text { minutes at ambient temperature } \\
\text { 3. Neutralize to } \mathrm{pH} \text { of } 7.5 \text { (record final } \mathrm{pH} \text { ) } \\
\text { 4. Spike with yeast extract concentrate to obtain a final concentration } \\
\text { of } 1000 \mathrm{ppm} \\
\text { 5. Incubate for } 2 \text { hours at ambient temperature } \\
\text { 6. Proceed with ATP assay procedures }\end{array}$ & $89 \%$ \\
\hline
\end{tabular}

$*$ percent $A T P$ reduction $=1-\frac{\text { ATP in ef fluent befors UV irradiation }}{A T P \text { in effluent after UV irradiation }} \times 100 \%$ 


\section{CHAPTER 4}

\section{HYPOTHESES}

This section outlines the hypotheses to be further examined in subsequent sections.

- ATP assay can be used as a monitoring tool for microbial contamination in water supplies.

- ATP assay results are not significantly influenced by sample turbidity.

- Chlorine disinfection oxidizes cellular ATP which can be detected by the ATP assay.

- ATP assay can be used as a monitoring tool for chlorine disinfection.

- UV irradiation decreases cellular activity which can be detected by the ATP assay.

- ATP assay can be used as a monitoring tool for UV disinfection.

- The sensitivity and detection limit of the ATP assay can be improved by modifying assay procedures. 


\section{CHAPTER 5}

\section{Materials AND Methods}

The purpose of this study was to test the applicability of the ATP luminescence assay as a monitoring and process optimization tool for water and wastewater applications. To accomplish this aim, a series of controlled experiments were performed to compare the results obtained from the ATP luminescence assay to those of the membrane filtration technique, which is a reliable and accepted enumeration method for bacteria.

The materials and methodology needed to accomplish this study are further described in this chapter. The experimental protocols common to all experimental phases, included a description of the statistical analysis employed for this study are described in Sections 5.1 to 5.6. Following, the phase-specific experimental procedures are described in Section 5.7 where the experimental design is comprised of four main phases:

- Preliminary Phase: Quality Control and Assurance aimed to understand the ATP assay, to test the effect of turbidity on the assay and to assure that the light measured was produced by the reaction between ATP and the Luminase reagents and not from background sources (Section 0).

- Phase I: Optimization Experiments examined methods to increase the sensitivity of the ATP (5.7.2). 
- Phase II: UV Disinfection Experiments aimed to confirm the initial field trials performed by LuminUltra and further examine the use of the ATP assay as a monitoring tool for UV disinfected water and wastewater (Section 5.7.3).

- Phase III: Chlorine Disinfection Experiments examined the use of the ATP assay as a system optimization and monitoring tool to be used with chlorine disinfected water and wastewater (Section 5.7.4).

\subsection{Cleaning and Sterilization Practices}

Before any set of experiments were performed, the following cleaning and sterilization practices were observed:

- Work surfaces, pipetters, tweezers and magnetic stirrers were cleaned with a 70 percent ethanol solution.

- Glassware, such as beakers, graduated cylinders, membrane filtration units and flasks were washed using a laboratory manual washing detergent, rinsed well with tap water to remove all soap residues, rinsed a second time with deionized water and allowed to dry.

- For sterilization, glassware was either exposed to ultraviolet light for 5 minutes or autoclaved for 15 minutes at $121^{\circ} \mathrm{C}$ and allowed to cool before use.

- Pipette tips were autoclaved for 15 minutes at $121^{\circ} \mathrm{C}$ before use.

- Tools frequently used during an experimental procedure, such as the tweezers that are used during membrane filtration (see Section 5.4), and the pipetters were also routinely sterilized between measurements. 


\subsection{Growth Media AND Base Solutions}

Frequently used media and solutions that were needed during all experimental phases included: the tryptic soy broth used to grow E.coli cultures, the agar medium used to enumerate the E.coli, and the phosphate buffered solution (PBS) and the $0.1 \%$ peptone water used as a base (dilution medium) for the surrogate solution. For all experiments, deionized water was dispensed by the Direct-Q UV3 unit by Millipore (Etobicoke, ON).

\subsubsection{SOY BROTH PREPARATION}

Growth media selected for the E.coli culture was a soybean casein digest medium called Bacto $^{\mathrm{TM}}$ Tryptic Soy Broth by BD Biosciences (Mississauga, ON). To prepare the medium, $3 \mathrm{~g}$ of Bacto ${ }^{\mathrm{TM}}$ Tryptic Soy Broth powder was added to $100 \mathrm{~mL}$ of deionized water. To dissolve the powder, the mixture was heated and mixed magnetically. Before use, the mixture was autoclaved for 15 minutes at $121^{\circ} \mathrm{C}$ and allowed to cool to room temperature.

\subsubsection{Agar Media Preparation}

M-Endo agar-Difco ${ }^{\mathrm{TM}}$ LES by BD Biosciences (Mississauga, ON) was the media selected as the growth medium for enumeration of coliform bacteria with membrane filtration (see Section 5.4). To prepare the agar, 10.2g of M-Endo agar was dissolved in $200 \mathrm{~mL}$ of deionized water, of which two percent by volume was replaced with $95 \%$ ethanol. The mixture was then heated while being stirred magnetically until boiling. The heated mixture was allowed to cool to $60^{\circ} \mathrm{C}$ before dispensing in $47 \mathrm{~mm}$ Petri dishes. Filled dishes were stored at $4^{\circ} \mathrm{C}$ in the refrigerator for up to two weeks. 


\subsubsection{BASE SOLUTION PREPARATION}

Phosphate buffered solution (PBS) and $0.1 \%$ peptone water are both suitable for bacteriological testing, as stated in Standard Methods (American Public Health Association (APHA), American Water Works Association (AWWA), and Water Environment Federation (WEF) 2005), and were seeded with E.coli. These solutions eliminated many unknowns, allowed for consistent analysis and an equal comparison for both the membrane filtration and ATP assay.

\subsubsection{Phosphate Buffer Solution (PBS) Preparation}

Phosphate buffered solution (PBS) was one of the base solutions employed for the simulated water samples, and was prepared according to the recipe provided in Standard Methods (APHA et al. 2005)

To make PBS, two stock solutions were required: a concentrated phosphate buffered water and a magnesium chloride solution. To prepare the concentrated phosphate buffered water, $34 \mathrm{~g}$ of potassium phosphate, $\mathrm{KH}_{2} \mathrm{PO}_{4}$, Fisher Scientific (Fair Lawn, NJ, USA) were dissolved in $500 \mathrm{~mL}$ of deionized water and the $\mathrm{pH}$ was adjusted to 7.2 with $1 \mathrm{~N}$ of sodium hydroxide $(\mathrm{NaOH})$. The solution was then diluted to $1 \mathrm{~L}$ with deionized water. The magnesium chloride solution was made by adding $81.1 \mathrm{~g}$ of magnesium chloride $\left(\mathrm{MgCl}_{2} \cdot 6 \mathrm{H}_{2} \mathrm{O}\right)$, Bishop Canada Inc (Burlington, $\mathrm{ON}$ ) to $1 \mathrm{~L}$ of deionized water.

Final PBS was prepared by adding $1.25 \mathrm{~mL}$ of the phosphate buffered water and $5.0 \mathrm{~mL}$ of magnesium chloride solution to $1 \mathrm{~L}$ of deionized water. Before use, PBS was autoclaved for 15 minutes at $121^{\circ} \mathrm{C}$ and allowed to cool to room temperature. 


\subsubsection{Peptone Water Preparation}

Peptone water was made according to the directions in Standard Methods (APHA et al. 2005). A concentrated peptone solution was first made by dissolving $10.0 \mathrm{~g}$ of Tryptone by EMD Chemicals Inc. (Germany) in $1 \mathrm{~L}$ of deionized water by heat. The concentrated solution was then dissolved to a final concentration of $0.1 \%$ of peptone (weight/volume).

\subsection{E. Coli Culture Preparation}

For all experiments, the E.coli strain ATCC $\left(23631^{\mathrm{TM}}\right.$ (American Type Culture Collection) obtained from Cedarlane Laboratories (Horny, ON) was used. The initial strain, received in pellet form, was rehydrated by Kerim Kollu in 2007 following the procedures outlined by the supplier. For further details regarding the rehydration of the E.coli pellets, please see Effects of Particles and Bioflocculation on Ultraviolet Disinfection (Kollu 2008).

A primary stock of E.coli was kept in the freezer in $2 \mathrm{~mL}$ Eppendrof Tubes by Eppendorf (Mississauga, ON). For each set of experiments, a new suspension of E.coli was prepared from the primary frozen stock. To do so, the frozen culture was revived overnight at $37^{\circ} \mathrm{C}$ in $100 \mathrm{~mL}$ of tryptic soy broth (see Section 5.2 .1 for the preparation of the broth). This suspension was subsequently incubated for 4 hours at $37^{\circ} \mathrm{C}$ in a ratio of $1: 100$ with soy broth. This method of preparation allows for the E.coli suspension to reach a growth phase that was in between the late exponential growth and the early stationary phases. 


\subsection{Membrane Filtration Procedure}

Membrane filtration was selected as the conventional bacteria enumeration technique, which offers a basis of comparison to the results of the ATP assay. The membrane filtration procedure, the Standard Total Coliform Membrane Filter Procedure (Protocol 9222 B), was followed as outlined in Standard Methods (APHA et al. 2005).

Membrane filtration units by Advantec (Japan) were used and consisted of: a glass cylinder, a porous media platform and a stainless steel clamp. To complete the experimental set-up, the platform was placed over a filter flask that was connected to the Vacuum Pressure Pump by Barnant Co, model number 400-1901 (Barrington, IL, USA). A $0.45 \mu \mathrm{m}, 47 \mathrm{~mm} \mathrm{S-Pak}{ }^{\mathrm{TM}}$ membrane filter by Millipore (Billerica, MA, USA) was gently placed over the porous media platform with smooth stainless steel forceps. The membrane glass cylinder was then placed over the platform and clamped in place. A Drummond Pipette Aid (Broomall, PA, USA) was used to place the required amount of experimental sample dilutions into the glass cylinder. The filtration of the sample was completed by suction by turning on the pump.

Experimental samples were diluted with PBS, to the required dilution, to obtain between 30 and 200 colonies per plate after incubation. Sample dilutions were performed in multiples of 10 with use of glass graduated cylinders. To assure complete mixture of the sample volume in the diluent volume, the final dilution volumes were mixed by inverting the graduated cylinder a minimum of 20 times. 
Once filtration of the sample was complete, the pump was turned off, the membrane filter was removed from the platform with the forceps and placed on an agar-filled Petri dish. The dishes were placed in the Isotemp Incubator by Fisher Scientific (Ottawa, ON), at $35^{\circ} \mathrm{C} \pm 5^{\circ} \mathrm{C}$ for 24 hours \pm 3 hours.

Periodically, the stock and dilution solutions were filtered to assure that these solutions were free from coliform bacteria contamination. These solutions included PBS, concentrated phosphate buffered water and a magnesium chloride stock solution as well as the $0.1 \%$ and stock solution of peptone water.

\subsection{ATP LUMINESCENCE ASSAY}

Quench-Gone $^{\mathrm{TM}}$ Aqueous Formulation, or QGA, from LuminUltra Technologies Ltd. (Fredericton, NB) was the selected adenosine triphosphate (ATP) luminescence assay as it was advertized to have the ability to detect very low levels of bacteria in water samples. QGA testing procedure was reported to be suitable for low-solids and low-biomass water such as: cooling water, drinking water, fresh water, ultrapure water and reuse water (LuminUltra Technologies 2010). The QGA test kit is also able to detect between 0.1 and $667,000 \mathrm{pg}$ ATP/mL (LuminUltra Technologies 2010).

As described in Chapter 3, QGA bioluminescent assay employs a light producing reaction that involves the ATP molecule and two compounds extracted from fireflies: luciferin and luciferase. A luminometer is a photometer that is able to detect and quantify low light levels such as those produced from the aforementioned reaction. The luminometer recommended by LuminUltra and selected for use with the QGA testing kit is the Kikkoman Lumitester C-110 (Japan). Light levels of the Kikkoman and other luminometers were measured in non-standardized relative light units, or RLU. To 
standardize the readings, a 1-ng $\mathrm{ATP} / \mathrm{mL}$ solution by LuminUltra Technologies (Fredericton, NB), called UltraCheck $1^{\mathrm{TM}}$, was used to determine the RLU measured per amount of ATP. The standardization step was also important because it was a measure of the reactivity of the Luminase and Luminase XXL reagents. These reagents contained luciferase, an enzyme that degrades over time. The standardization step was performed before every set of experiments.

The specifications of the Kikkoman Lumitester C-110 state that the limits of the instrument were $4 \times 10^{-12}$ to $1 \times 10^{-6}$ moles/L of ATP (Kikkoman Corporation). Using the equation of the default calibration curve provided by Kikkoman, see Equation 5.1, where $y$ was the concentration of ATP in moles/L and $x$ was the measured RLU, the range of the Lumitester C-110 can be converted to 20 to $5 \times 10^{6}$ RLU.

$$
y=2 \times 10^{-13} x
$$

Source: (Kikkoman Corporation)

QGA was designed to measure the concentration of cellular ATP. To do so, microorganisms are captured on a glass microfiber filter. The filtration step also concentrates the water sample to allow for increased sensitivity. The captured cells are lysed to release the cellular ATP. Light produced from the reaction between the cellular ATP and a mixture of luciferin, luciferase and magnesium is recorded by the luminometer (LuminUltra Technologies 2010a). 


\subsubsection{ATP LUMINESCENCE ASSAY MATERIALS}

The following consumables and reagents are needed to carry out the ATP assay:

- $60 \mathrm{~mL}$ Syringe with luer-lok

- $25 \mathrm{~mm}$ diameter and $0.7 \mu \mathrm{m}$ pore size glass microfiber membranes with luer-loc

- $17 \mathrm{~mm}$ diameter and $100 \mathrm{~mm}$ long medium culture tubes with caps

- $12 \mathrm{~mm}$ diameter and $55 \mathrm{~mm}$ long small polyphenylene (PPE) culture tubes

- 10 to $100 \mu \mathrm{L}$ pipette tips

- 100 to $1000 \mu \mathrm{L}$ pipette tips

- 1 to $5 \mathrm{~mL}$ pipette tips

- UltraLyse7 (LuminUltra, Fredericton NB)

- UltraLute (LuminUltra, Fredericton NB)

- UltraCheck1 (LuminUltra, Fredericton NB)

- Luminase or XXL Luminase (LuminUltra, Fredericton NB)

- Note: Luminase and XXL Luminase must be stored at $4{ }^{\circ} \mathrm{C}$ and allowed to reach room temperature before use.

The non-consumable materials required include:

- Kikkoman Lumitester C-110 single tube luminometer (Japan)

- 10 to $100 \mu \mathrm{L}$ pipetter

- 100 to $1000 \mu \mathrm{L}$ pipetter

- 1 to $5 \mathrm{~mL}$ pipette 


\subsubsection{COST OF THE ATP LUMINESCENCE ASSAY}

Materials needed to complete the QGA luminescence assay can be purchased from LuminUltra Technologies (Fredericton, NB). A QGA test kit comes complete with the consumable materials and reagents needed to complete 100 individual assays and costs approximately $\$ 870$ is using Luminase and $\$ 1,050$ (or $\$ 8$ to $\$ 10$ per individual replicate) (LuminUltra Technologies 2010b). Non-consumable materials include the luminometer, which can be purchased from LuminUltra Technologies, and three pipetters. The luminometer is valued at $\$ 4,300$ (LuminUltra Technologies $2010 \mathrm{~b}$ ) and the price for three pipetters is approximately $\$ 850$ (Thermo Fisher Scientific 2007). The start-up cost is thus just below $\$ 5,000$.

\subsubsection{ATP LUMINESCENCE ASSAY PROCEDURE}

There are four main steps needed to complete the QGA test procedure. These steps are presented below however, as mentioned in Section 5.5, a calibration step must be performed before the testing procedure can be completed. To determine the strength of the Luminase (or XXL Luminase) reagent and to standardize the luminometer, $100 \mu \mathrm{L}$ of the Luminase (or XXL Luminase) reagent was added to $100 \mu \mathrm{L}$ the UltraCheck1 solution in a small culture tube. The tube was gently swirled for 5 seconds and placed in the luminometer to obtain a reading. This value was later be used to convert the reading from the luminometer (measured in RLU) to the amount of ATP (measured in $\mathrm{pg} / \mathrm{mL}$ ) present in the samples, see Section 5.5.4.

The four steps to complete the QGA test procedure are: filter, lyse, dilute, and measure. A description of these steps follows. For additional details please see the QGA Product Fact Sheet (LuminUltra Technologies 2010a): 
1. Filter: The syringe was prepared for filtration by removing the plunger and attaching the filter. The sample was then poured into the barrel of the syringe after which the plunger was inserted. Pressure was applied to the plunger to pass the sample through the filter. To release the filter from the syringe, the filter was unscrewed before pulling out the plunger. This released the pressure that has formed inside the barrel of the syringe. This process was repeated until the desired sample volume had been filtered. The surface of the filter retains the microbial cells.

2. Lyse: To lyse the cells collected on the filter, the filter was re-attached to the syringe and $1 \mathrm{~mL}$ of UltraLyse 7 was added to the barrel. The syringe was placed over a medium culture tube and the plunger was used to push all contents into the culture tube.

3. Dilute: To dilute the sample, $9 \mathrm{~mL}$ of UltraLute was added to the medium culture tube. The tube was then capped and inverted gently five times to mix.

4. Measure: In a small culture tube, $100 \mu \mathrm{L}$ of Luminase (or XXL Luminase) and $100 \mu \mathrm{L}$ of the diluted solution was added and swirled gently, by hand for 5 seconds to mix. The small culture tube was then placed in the luminometer and the measured RLU was measured and recorded.

The size of the sample volume depends on the type of water that is being analyzed but can range between $5 \mathrm{~mL}$ and $100 \mathrm{~mL}$. Due to the high bacteria concentrations (in the order of $10^{5} \mathrm{CFU} / \mathrm{mL}$ ) of our sample solution, initial experiments were performed with sample volumes of $5.0 \mathrm{~mL}$. This sample size was later revised and will be further 
discussed in Chapter 6. Thorough this study, a minimum of two to five replicates were carried out during this study.

\subsubsection{RLU TO MASS OF ATP CALCULATIONS}

To convert the RLU values obtained from the luminometer to the amount of ATP in the sample the value obtained from the calibration step was needed. The calculation to perform follows:

$$
A T P_{\text {sample }}\left(\frac{p_{g} A T P}{m L}\right)=\frac{R L U_{\text {sample }}(R L U)}{R L U_{\text {UCL }}\left(\frac{R L U}{\frac{1 m g I P}{m L}}\right) \times 0_{.1} 1(m L)} \times \frac{10^{-9}(n g)}{10^{-12}(p g)} \times \frac{1}{V_{\text {gample }}(m L)}
$$

which, can be simplified to:

$$
A T P_{\text {sample }}\left(\frac{p g A T P}{m L}\right)=\frac{R L U_{\text {sample }}(R L U)}{R L U_{U C 1}(R L U)} \times \frac{10,000(p g A T P)}{V_{\text {sample }}(m L)}
$$

where $A T P_{\text {sample }}$ is the amount of ATP, measured in $\mathrm{pg} \mathrm{ATP} / \mathrm{mL}$, in the volume of the filtered sample, $R L U_{\text {sample }}$ is the value read from the luminometer, $R L U_{U C l}$ is the value read from the luminometer during the calibration step, $10,000 \mathrm{pg} A T P$ is a conversion factor that considers the volume of UltraCheck 1 used for the calibration $(100 \mu \mathrm{L}$ or 0.1 $\mathrm{mL}$ ) and the conversion from ng to $\mathrm{pg}$, and $V_{\text {sample }}$ is the volume of the sample that was filtered measured in $\mathrm{mL}$. 


\subsection{Statistical Analysis}

The following describes the statistical analysis performed, when necessary, on the results of the experimental phases. Microsoft Excel (San Leandro, CA) was used to perform simple statistical analysis such as calculating the sample mean, the standard deviation and performing simple T-tests. More complex statistical analysis was performed with a software add-in package for Excel called XLSTAT (New York, NY).

\subsubsection{CALCULATING THE SAMPLE MEAN AND THE SAMPLE VARIANCE}

If three or more replicates were performed than the average value of the replicates was calculated and presented along with the standard deviation, which represents the spread of the data (Currell and Dowman 2005). If two replicates were performed, the average of the values was still reported but reporting of the standard deviation was not appropriate. The average was calculated as follows:

$$
\bar{x}=\frac{\sum_{1}^{n}\left(x_{i}\right)}{n}
$$

where, $\bar{x}$ was the sample mean, $n$ was the total number of samples measured and $x$ was

the value of individual replicates (Currell and Dowman 2005). The sample variance, also known as the standard deviation, was calculated as follows:

$$
s=\sqrt{\frac{\sum_{1}^{n}\left(x_{i}\right)^{2}-\frac{\left(\sum_{1}^{n} x_{i}\right)^{2}}{n}}{n-1}}
$$

where, $s$ was the standard deviation of the sample, $\mathrm{n}$ was the total number of samples measured and $\mathrm{x}$ was the value of individual replicates (Currell and Dowman 2005). 


\subsubsection{TWO-SAMPLE T-TEST}

T-statistics were used to determine if the sample means, $\bar{x}_{a}$ and $\bar{x}_{b}$, of two different two samples sets are from the same or different populations. In other words, a $t$-test was performed to determine if two sample sets were significantly different from each other (Currell and Dowman 2005).

The null hypothesis, $\mathrm{H}_{0}$, of a two-sample two-tailed $t$-test states that the population means, $\mu_{a}$ and $\mu_{b}$, of two sample sets are equal and the alternative hypothesis, $\mathrm{H}_{\mathrm{A}}$, is that the populations means of the two samples sets were not equal (Currell and Dowman 2005):

$$
\begin{gathered}
\boldsymbol{H}_{\mathbf{0}}: \boldsymbol{\mu}_{\boldsymbol{a}}=\boldsymbol{\mu}_{\boldsymbol{b}} \\
\boldsymbol{H}_{\boldsymbol{A}}: \boldsymbol{\mu}_{\boldsymbol{a}} \neq \boldsymbol{\mu}_{\boldsymbol{b}}
\end{gathered}
$$

The estimated probability of making a type I error (also known as $p$-value or simply $p$ ) of two-sample two-tailed $t$-test was performed in Excel where a type I error is defined as incorrectly accepting the null hypothesis. The significance level, $\alpha$, for all experiments was set at 0.05 . The significance level is the maximum acceptable probability of making a type I error. The null hypothesis was accepted if the p-value was greater than the significance level. Otherwise, the alternative hypothesis was accepted (Currell and Dowman 2005). 


\subsubsection{ANALYSIS OF VARIANCE (ANOVA) AND POST ANOVA TESTS}

When more than two sample sets were to be compared a one-way analysis of variance, or ANOVA, was performed. The one-way ANOVA test uses F-statistics to compare the variances between and within sample sets (Currell and Dowman 2005). The software addin package XLSTAT was used to complete the test (Addinsoft 2010).

Similar to the two-tailed $t$-test, the significance level, $\alpha$, was set at 0.05 and the alternative hypothesis was accepted if the $p$-value was below the significance level. Otherwise, the null hypothesis was accepted. The null hypothesis was that there were no differences of the treatment applied and the alternative hypothesis was that there were differences (Currell and Dowman 2005). If the alternative hypothesis was accepted, then additional tests were required testing to determine where the differences lie (Currell and Dowman 2005). The alternative tests employed for this study include Dunnett and Tukey test, which were also completed with the XLSTAT (Addinsoft 2010).

Dunnett test compares each sample-set to that of the blank to determine if there was a significant difference between each pair of data-sets compared. The Tukey test makes pair-wise comparisons between each sample-sets (including the blank) to determine if there was a significant difference between each pair of data-set compared. As aforementioned statistical analysis, the output of the tests report the $p$-value, which was compared to the significance level of 0.05 to determine if there was significant difference between the pair of data-sets compared (Addinsoft 2010). 


\subsection{Phase Specific Experimental Protocols}

The experimental procedures employed for the different experimental phases are presented in the following sections. The tasks completed for each experimental phase are summarized in Table 5.1. Unless otherwise noted, the initial sample solution was prepared by adding $50 \mu \mathrm{L}$ of E.coli stock solution to $500 \mathrm{~mL}$ of base solution (either $0.1 \%$ peptone water or PBS). 
Table 5.1: Experimental tasks completed for each of the four experimental phases

\begin{tabular}{|c|c|}
\hline Experimental Phase & Task Completed \\
\hline $\begin{array}{l}\text { Preliminary Phase } \\
\text { Quality Control and } \\
\text { Assurance }\end{array}$ & $\begin{array}{l}\text { The quality control and assurance tests included the completion of: } \\
\text { - the light leakage and background levels of the luminometer and } \\
\text { test tubes; } \\
\text { - the background light levels of deionized water and peptone water; } \\
\text { - the repeatability of the ATP assay; } \\
\text { the effect of the turbidity of surface water on the results of the } \\
\text { ATP assay; } \\
\text { initial calibration curves for low bacteria concentrations with } \\
\text { Luminase, and; } \\
\text { initial calibration curve for high bacteria concentrations with } \\
\text { Luminase. }\end{array}$ \\
\hline $\begin{array}{l}\text { Phase I } \\
\text { Optimization of the } \\
\text { ATP Assay }\end{array}$ & $\begin{array}{l}\text { Determination of optimization of the ATP assay included: } \\
\text { - the effect of varying pore size of the filter of the ATP assay; } \\
\text { - the effect of varying the amount of Lysing solution used with the } \\
\text { ATP assay. } \\
\text { the completion of a calibration curve for low bacteria } \\
\text { concentrations with XXL Luminase } \\
\text { the completion of a calibration curve for high bacteria } \\
\text { concentrations with Luminase. }\end{array}$ \\
\hline $\begin{array}{l}\text { Phase II } \\
\text { UV Experiments }\end{array}$ & $\begin{array}{l}\text { Assessment of the ATP assay as a tool to verify the effectiveness of UV } \\
\text { irradiation included the determination of: } \\
\text { - UV dose response curves with Luminase and XXL Luminase; } \\
\text { - the effect of incubation time on the reduction of ATP subsequent } \\
\text { to UV irradiation; } \\
\text { - the effect of incubation temperature on the reduction of ATP } \\
\text { subsequent to UV irradiation, and; } \\
\text { the effect of pretreatment on the reduction of ATP subsequent to } \\
\text { UV irradiation }\end{array}$ \\
\hline $\begin{array}{l}\text { Phase III } \\
\text { Chlorine Experiments }\end{array}$ & $\begin{array}{l}\text { Evaluation of the use of the ATP assay as a monitoring tool for chlorine } \\
\text { disinfection included an assessment of: } \\
\text { - The use of the ATP assay with Luminase, and; } \\
\text { - The use of the ATP assay with XXL Luminase }\end{array}$ \\
\hline
\end{tabular}




\subsubsection{Preliminary PHASE: QUALITy ConTrol AND ASSURANCE}

The purpose of the preliminary phase was to assure that the light measured by the luminometer was not the source of instrument error or background sources. In addition this phase aimed to make preliminary observations of the correlation between the bacteria concentration and the measured amount of ATP in solution. The procedures needed to complete this testing phase are further described in this section. Please note that the procedures for the background light levels and light leakage tests.

\subsubsection{Light Leakage into the Luminometer}

Light leakage into the Kikkoman Lumitester C-110 (Japan) was completed by measuring the light reading of:

- the empty chamber;

- an empty small test tube;

- $100 \mu \mathrm{L}$ of UltraChequel in a small test tube, and;

- the mixture of $100 \mu \mathrm{L}$ of Ultracheque1 and Luminase.

UltraChequel is a solution of $1 \mathrm{ng} \mathrm{ATP/mL}$ and in the presence of Luminase should produce light. The average light reading of three replicates was reported.

\subsubsection{Background Light Level Measurements}

Background light level measurements of reagents and solutions were performed following the ATP assay procedure outlined in Section 5.5 with the exception that either no sample volume was filtered, or the sample volume was replaced with ether deionized water or a base solution. 


\subsubsection{Turbidity Measurements}

Turbidity measurements were performed with the Hach 2100AN Turbidity meter.

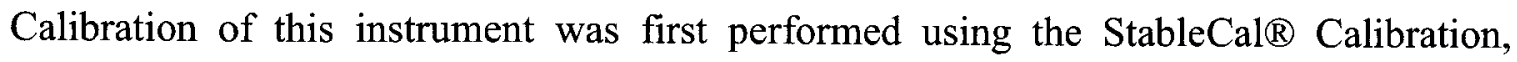
following the procedure outlined in the instruction manual (Hach 2006). To complete a turbidity measurement, the interior of a sample cell is rinsed with deionized water before a sample was added to the cell. The exterior of the cell was then rinsed with deionised water and dried. A small bead of silicone oil was then applied to the cell and wiped with a lint-free cloth. The sample cell was then placed in the turbidity meter and a turbidity measurement was taken.

To complete the turbidity experiments, a sample of the Rideau River near the Stacie Building on Carleton University's campus (Ottawa, ON) was collected and immediately measured. In order to determine the effect of turbidity on ATP readings, the turbidity of the river was incrementally increased by adding a clay suspension solution. To make the clay solution, 2.5 grams of bentonite clay was added to 200 millilitres of deionized water. Volumes of $0.5,0.7,1.0,1.5$, and 2.0 millilitres of the clay suspension solution was added to 300 millilitres of river water and allowed to mix magnetically for one minute. Subsequently, turbidity measurements were done in triplicate and the average turbidity measurement was reported. 


\subsubsection{Calibration Curves}

Calibration curves were created to show the response of the ATP assay to solutions of known bacterial concentrations (Harris 2003). For these experiments, a series of sample dilutions were prepared and tested in parallel by the ATP assay and membrane filtration.

For calibration curves that examined low bacteria concentrations $\left(<10^{5} \mathrm{CFU} / \mathrm{mL}\right)$, the initial experimental solution was prepared by adding $50 \mu \mathrm{L}$ of E.coli stock (see Section 5.3) to $500 \mathrm{~mL}$ 0.1\% solution of peptone water (see Section 5.2.3.2) of PBS (see Section 5.2.3.1). This dilution typically yielded a microbial of $10^{5} \mathrm{CFU} / \mathrm{mL}$. The number of dilutions varied for each calibration curve performed and additional details are provided in Chapter 6. For calibration curves that examined high bacteria concentrations $\left(>10^{5}\right.$ $\mathrm{CFU} / \mathrm{mL}$ ) the initial experimental solution was the non-diluted E.coli stock solution (see Section 5.3) and subsequent dilutions were prepared directly from this stock solution.

For calibration curves using small sample volumes $(<250 \mathrm{~mL})$, dilutions were prepared using Erlenmeyer flasks, which were sterilized in the autoclave for 15 minutes and allowed to cool to room temperature before use.

Calibration curves using larger samples (> $250 \mathrm{~mL}$ ) employed large beakers with autoclaveable lids with $500 \mathrm{~mL}$ or $1 \mathrm{~L}$ capacities (Pyrex, Germany). The beakers were first filled with PBS or 0.1\% peptone water (see Section 5.2.3.1 and Section 5.2.3.2 respectfully) autoclaved for 15 minutes and allowed to cool to room temperature before use. These solutions were then measured to the required volume and the appropriate amount of experimental solutions was added to obtain the desired dilution. 
Unless otherwise stated, three replicates of each the ATP assay and the membrane filtration technique were performed for each dilution. When not being tested, dilution solutions were kept in the fridge (at $4^{\circ} \mathrm{C}$ ) covered with para-film and foil, to prevent growth. In all instances, the ATP assay was performed first and membrane filtration second. Before any sampling or measurement of a solution was performed it was well mixed by inverting the sample for 10 seconds.

\subsubsection{PHASE I: OPTIMIZATION EXPERIMENTS}

Preliminary experiments indicated that this particular ATP assay (QGA kit) was not as sensitive as it was advertized. The purpose of this experimental phase was to increase the efficiency of the ATP luminescence assay and improve its sensitivity by modifying some of the procedures.

The first experiment aimed to increase the amount of bacterial cells captured by decreasing the pore size of the filter from $0.7 \mu \mathrm{m}$ to $0.2 \mu \mathrm{m}$ (Section 5.7.2.1). The second experiment aimed to increase the effectiveness of the lysing step by increasing the amount of lysing solution from $1 \mathrm{~mL}$ to $2 \mathrm{~mL}$ and $3 \mathrm{~mL}$ (5.7.2.2). The third experiment aimed to increase the amount of bacterial cells captured further increasing the amount of light produced by increasing the volume of the samples filtered and using XXL Luminase. The latter set of observations was made using calibration curves, as described in Section 5.7.1.4. 


\subsubsection{Effect of Varying the Pore Size of the Filter}

To complete this experiment, an initial experimental solution of $50 \mu \mathrm{L}$ of E.coli stock solution was introduced to $500 \mathrm{~mL}$ of PBS. From this solution, called Sample 1, two ATP test were performed in parallel and each in triplicate. The first series of tests followed the normal protocol of the ATP assay, see Section 5.5, using $50.0 \mathrm{~mL}$ of Sample 1.

The second series of tests also followed the ATP assay procedures (see Section 5.5), however a $0.2 \mu \mathrm{m}$ pore size filter was used instead of a $0.7 \mu \mathrm{m}$ pore size filter. In addition, the volume of Sample 1 filtered was decreased to $5.0 \mathrm{~mL}$. It was initially hoped that each ATP test would use $50.0 \mathrm{~mL}$ sample volumes, however, the $0.2 \mu \mathrm{m}$ pore size filter required a great amount of manual effort to filter. As a result, the volume for this test was reduced to $5.0 \mathrm{~mL}$.

Both series of tests were duplicated using PBS and a membrane filtration of both the pure PBS solution and of Sample 1 was also performed (see Section 5.4).

\subsubsection{Effect of Varying the amount Lysing Solution}

For this series of experiments, three ATP assays were performed with an experimental solution prepared from $50 \mu \mathrm{L}$ of E.coli stock solution added to $500.0 \mathrm{~mL}$ of $0.1 \%$ peptone water. The ATP assays followed the procedures outlined in Section 5.5 however each assay varied the amount of lysing solution. The three volumes of lysing solution added were: $1 \mathrm{~mL}, 2 \mathrm{~mL}$ and $3 \mathrm{~mL}$. Measurements were taken immediately after completing the test (as per the normal operating procedures) and approximately 5 minutes after introducing the lysing solution. 


\subsubsection{PHASE II: UV INACTIVATION EXPERIMENTS}

The experimental set-up and procedures followed for the UV deactivation experiments are those outlined by Bolton and Linden (2003). A bench-scale collimated beam apparatus was used, see Figure 5.1, with four low-pressure mercury lamps (Phillips UV-C germicidal lamps). The lamps provided nearly-monochromatic UV light at $253.7 \mathrm{~nm}$. Two apertures were placed below the lamps to focus the beam that reached the sample placed on the irradiation stage. The inside of the apparatus was painted with a matt black paint to assure little to no reflectivity from surfaces. The irradiation stage was a magnetic stir plate 200 Mini Stirrer by VWE Scientific Products (West Chester, PA, USA). During irradiation, the sample was stirred with a micro stir bar to assure that the sample was well mixed and to avoid vortex mixing (Bolton and Linden 2003).

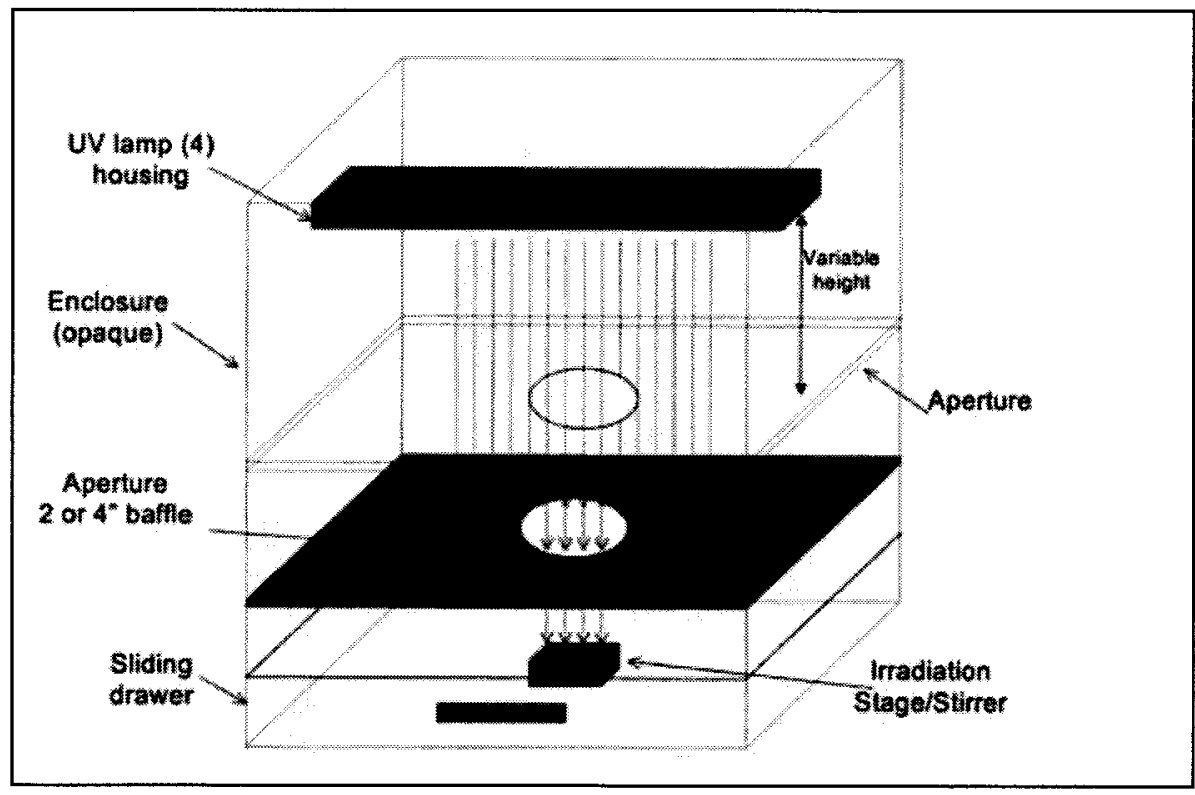

Figure 5.1: UV Collimated Beam Set-Up (source: Bolton and Linden 2003) 


\subsubsection{Calculating the UV Dose}

Germicidal fluence, or UV dose, is the amount of irradiation that a sample has been exposed to. Throughout the experiments, six UV doses were tested: $0,20,40,60,80$ and $100 \mathrm{~J} / \mathrm{m}^{2}$. The UV dose is defined as follows:

$$
\text { UV Dose }\left(J / \mathrm{m}^{2}\right)=E_{\text {avg }}^{\prime}\left(W / m^{2}\right) \times t(s)
$$

where the average germicidal fluence rate, $E^{\prime}{ }_{\text {avg }}\left(\mathrm{W} / \mathrm{m}^{2}\right)$, and the time of exposure, $t(\mathrm{~s})$, (Bolton and Linden 2003). To apply Equation 5.8, the desired UV dose was first selected, the average germicidal fluence rate calculated to determine the time of exposure, $t$, required for the selected UV dose.

The average germicidal fluence rate, $E_{\text {avg }}$ (see Equation 5.9), is the measured radiant power, $E_{0}\left(\mathrm{~W} / \mathrm{m}^{2}\right)$, that has been corrected for four factors: the Petri factor $\left(F_{p e t r i}\right)$, the reflection factor $\left(F_{\text {relection }}\right)$, the water factor $\left(F_{\text {water }}\right)$ and the divergence factor $\left(F_{\text {divergence }}\right)$. The average fluence rate is as follows (Bolton and Linden 2003):

$$
E_{\text {avg }}^{\prime}=E_{0} \times F_{\text {petri }} \times F_{\text {reflection }} \times F_{\text {water }} \times F_{\text {divergence }}
$$

The radiant power, $E_{0}\left(\mathrm{~W} / \mathrm{m}^{2}\right)$, was measured with a radiometer (International Light, MA, USA) at same height as the surface of the liquid to be irradiated.

The Petri factor is the average irradiance over the surface area of the dish and corrects for the variations of irradiance over the surface of the liquid in the dish (Bolton and Linden 2003). There were no observed differences in the irradiation field and the Petri factor was taken as unity. 
The reflection factor accounts for light that is reflected when it hits the surface of the liquid. For light between 200 and $300 \mathrm{~nm}$, the amount reflected, R, is 0.025 and the reflection factor, 1-R, and is equal to 0.097 (Bolton and Linden 2003).

The water factor accounts for the light that is absorbed by the water and is defined as:

$$
F_{\text {water }}=\frac{1-10^{-a l}}{a l \ln (10)}
$$

where $a$ is the absorbance for a $1-\mathrm{cm}$ path at $254 \mathrm{~nm}\left(\mathrm{~cm}^{-1}\right)$ and $l$ is the vertical path length $(\mathrm{cm})$ of the water in the dish (Bolton and Linden 2003). The absorbance was measured with a UV-visible spectrophotometer (Varian Model Cary 100BIO) that had been previously balanced with deionized water. The absorbance was measured for every new preparation of sample solution. The sample volumes to be irradiated remained constant throughout all testing at $20.0 \mathrm{~mL}$ that corresponds to a vertical path length of $0.603 \mathrm{~cm}$.

The divergence factor defined as:

$$
F_{\text {divergence }}=\frac{L}{(L+D)}
$$

where L is distance between the UV lamps and the surface of the sample $(\mathrm{cm})$ and $l$ is the vertical path length $(\mathrm{cm})$ (Bolton and Linden 2003). For the experimental set-up described here both $\mathrm{L}$ and $l$ remained constant at $48.5 \mathrm{~cm} 0.603 \mathrm{~cm}$ respectfully to give a divergence factor of 0.988 . 


\subsubsection{UV Inactivation Experimental Procedure}

To complete the irradiation of a sample, the UV dose was first selected and the time to achieve the UV dose was determined following the procedure outlined in Section 5.7.2.1.

A volume of $20.0 \mathrm{~mL}$ of the solution to be irradiated was placed in $150 \mathrm{~mL}$ Pyrex dish (Germany). A magnetic micro-stirrer was placed in the dish which was then placed on the on the irradiation stage/stirrer, see Figure 5.1. The stirrer was set at minimum. A piece of cardboard was placed above the bottom aperture, the door of the collimated apparatus was closed and the UV light was turned on. Approximately 30 seconds was allowed to elapse before the cardboard was removed and the timer started. The cardboard prevented any UV light to reach the sample while the lamps were equilibrating. Once the required irradiation time had elapsed, the light was promptly turned off and the sample was tested immediately by the required ATP assay, see Section5.5, and/or membrane filtration, see Section 5.4.

\subsubsection{Pretreatment Method}

To be able to use the ATP assay to verify the effectiveness of UV irradiation, there must be a significant decrease in the quantities of ATP measured before and after UV irradiation. To improve the performance of the ATP assay, preliminary trials performed by LuminUltra Technologies tested a total of four pretreatment methods that showed a percent reduction in ATP that ranged from $14 \%$ to $89 \%$, see Table A. 1 (LuminUltra Technologies). The most successful pretreatment, the one which saw an $89 \%$ percent reduction in ATP, was attempted and is described here. 
The pretreatment was completed as followed:

- A volume of $1.0 \mathrm{~mL}$ of LumiSolveX10 was added for every $9 \mathrm{~mL}$ of experimental solution. For a $20.0 \mathrm{~mL}$ sample, $2.2 \mathrm{~mL}$ of LumiSolveX10 was added.

- The solution was then incubated for 30 minutes at room temperature.

- The solution was neutralized to $\mathrm{pH}$ of 7.5. The $\mathrm{pH}$ was recorded before and after neutralization.

- A volume of $2.47 \mathrm{~mL}$ of a $10 \mathrm{~g} / \mathrm{L}$ yeast extract solution was added to the sample to achieve a final yeast concentration of $1000 \mathrm{ppm}$.

- The sample was incubated for 2 hours at room temperature.

After the incubation period had elapsed, $5.0 \mathrm{~mL}$ of the sample solution was measured with the ATP assay, see Section 5.5, and the required volume was measured following the membrane filtration method, see Section 5.4.

\subsubsection{PHASE III: CHLORINE DISINFECTION EXPERIMENTS}

Chlorine disinfection is measured the $\mathrm{CT}$ value which is the multiplication of the concentration $\mathrm{C}\left(\mathrm{mg} / \mathrm{L} \mathrm{Cl}_{2}\right)$ and the contact time, $\mathrm{t}(\mathrm{min})$. The chlorine disinfection experiments examined a total of three concentrations: 1,5 and $10 \mathrm{mg} / \mathrm{L} \mathrm{Cl}$. and four contact time: of $0,5,15,30$ and 45 minutes. The procedures needed to complete the chlorine experiments are outlined here.

The chlorine source was a solution of 5.65 to $6 \%(w / v)$ SS290 laboratory grade sodium hypochlorite by Fisher Scientific (New Jersey), NaOCL from which a chlorine stock solution of $1 \mathrm{~g} / \mathrm{L} \mathrm{Cl}_{2}$ was created. Sodium hypochlorite solutions are strong oxidants that can quickly degrade in the presence of heat or light. For this reason, both the initial 
solution and the stock solution were covered with foil, and stored at $4{ }^{\circ} \mathrm{C}$ (Metcalf \& Eddie 2003). The chlorine stock solution was used to achieve the initial chlorine concentrations.

The amount of total chlorine in solution was measured with the Hach DR2800 Spectrophotometer (Mississauga, ON) following method 8167 (Hach, n.d.) using DPD Total Chlorine Reagent for $10 \mathrm{~mL}$ sample by Hach Permachem Reagents (Germany). For chlorine concentrations of $1 \mathrm{mg} / \mathrm{L} \mathrm{Cl}$, sample volumes of $10.0 \mathrm{~mL}$ were used. For chlorine concentrations of 5 and $10 \mathrm{mg} / \mathrm{L} \mathrm{Cl}_{2}$, sample volumes of $1.0 \mathrm{~mL}$ were taken and diluted to $10.0 \mathrm{~mL}$ with PBS to stay within the limits of the spectrophotometer.

After the contact times have been observed, dechlorination was required to stop the chlorine from further reacting. Dechlorination is typically performed with sulphite compounds such as sodium sulphite $\left(\mathrm{Na}_{2} \mathrm{SO}_{3}\right)$ and sodium bisulfate $\left(\mathrm{NaHSO}_{3}\right)$. For these experiments, sodium bisulfate was selected. Sodium bisulfate has a molecular weight of $104.06 \mathrm{~g} /$ mole and, stoichiometrically, $1.465 \mathrm{mg}$ of sodium bisulfate was required for every $1 \mathrm{mg} / \mathrm{L}$ of chlorine residual. In practice, 1.5 to $1.7 \mathrm{mg}$ of sodium bisulphate per $\mathrm{mg} / \mathrm{L}$ of chlorine is employed (Metcalf \& Eddie 2003). A sodium bisulphate stock solution was created by dissolving $1.7 \mathrm{~g}$ of sodium bisulphate, $\mathrm{NaHSO}_{3}$, by Sigma Akdrich (St. Lousi, MO) in $100.0 \mathrm{~mL}$ if deionized water. The dechlorination reaction is as follows:

$$
\begin{gathered}
\mathrm{NaHSO}_{3}+\mathrm{Cl}_{2}+\mathrm{H}_{2} \mathrm{O} \rightarrow \mathrm{NaHSO}_{4}+2 \mathrm{HCl} \\
\text { source: (Metcalf \& Eddie 2003) }
\end{gathered}
$$




\subsubsection{Chlorine Experimental Procedures}

An initial experimental solution was made by adding the desired amount of E.coli to a base solution. Before chlorine was added, the initial bacterial concentration and amount of ATP was measured following the procedures outline in Section 5.4 and Section 5.5, respectively.

Two series of chlorine experiments were complete for this study, which differed slightly in the experimental design as well as the sample volume size and the type of Luminase reagent used. The first set of experiments was performed using a 1 L Pyrex beaker (Germany) that had been covered with foil. A magnetic stirrer was added the beaker, which was then placed on a Corning Stirrer Scholar 171 (Lowell, MA, USA). The moment the chlorine was added to the experimental solution, the timer was started. After each contact time, a sample was taken from the beaker to measure total chlorine in solution. Immediately afterwards, a second sample was taken from the beaker and dechlorinated with the sodium bisulphate stock solution and allowed to mix for a minimum of 5 minutes. To assure the effectiveness of dechlorination, the total chlorine was measured and should be $0 \mathrm{mg} / \mathrm{L} \mathrm{Cl}_{2}$. The bacteria concentration and the amount of ATP were then assessed using membrane filtration, see Section 5.4, and the ATP bioluminescence assay with Luminase and sample volumes of $5.0 \mathrm{~mL}$, see Section 5.5.

The second set of chlorine experiments were completed following the procedure detailed above with the exception that the experimental sample volume was increased, which allowed an increase sample volume to be filtered during the ATP assay. This set of experiments was completed using Phipps and Bird P13-70 Jartester set at $100 \pm 2$ RPM.

Five $1 \mathrm{~L}$ Pyrex beakers, one for each contact time, were covered with foil and filled with 
$500 \mathrm{~mL}$ of PBS to which $50 \mu \mathrm{L}$ of E.coli stock solution was added. As previously described, the total chlorine was measured at each contact time. Also at the completion of each contact time, the content of the appropriate beaker was dechlorinated. Following, the bacteria concentration and the amount of ATP were measured using membrane filtration, see Section 5.4, and the ATP bioluminescence assay with XXL Luminase and $100.0 \mathrm{~mL}$ sample volume, see Section 5.5. 


\section{CHAPTER 6}

\section{Results AND Discussion}

The purpose of this study was to evaluate the use of the ATP bioluminescence assay as a real-time monitoring and system optimization tool for surface water and UV inactivated and chlorinated wastewater. The following chapter outlines the results and discussion of experimental phase of this study.

This study was completed in four phases. The Preliminary Phase: Quality Control and Assurance examined the limits and characteristics of the ATP luminescence assay. This phase examined the accuracy and the sensitivity limits of the assay as well as of the effects of changing certain elements of the testing procedure (Section 6.1). During the preliminary phase, it became evident that optimization of the test procedures would be required to achieved the necessary sensitivity limits. Thus, in Phase I: Optimization of the ATP Assay measures to increase the sensitivity of the assay were examined (Section 6.2). During the next testing phase, Phase II: UV Inactivation Experiments, the applicability of the ATP assay for use as a disinfection monitoring tool for water and wastewater subsequent to UV disinfection was assessed (Section 6.3). This phase also included an examination of the pretreatment methods described by LuminUltra technologies (see Section 3). The final testing phase, Phase III: Chlorine Disinfection Experiments, assessed the applicability of the ATP luminescence assay as a disinfection monitoring tool for water and wastewater after chlorination (Section 6.4). 


\subsection{Preliminary Phase: Quality Control and Assurance}

The purpose of these experiments was to establish the quality control and assurance for the ATP test, verify the accuracy and reproducibility of results, and determine the sensitivity and the detection limits of the ATP assay. Once it became clear that limits of detection were not as low as advertized, the objectives of the testing phase were expanded to include modification of the assay procedures to improve the sensitivity of the test.

\subsubsection{LIGHT LEAKAGE AND BACKGROUND LIGHT PRODUCED FROM REAGENTS}

The light leakage and background light tests was performed to assure that the light measured with the luminometer was from the reaction between Luminase and ATP and not from an outside source leaking into the instrument, or caused by background levels of the reagents.

\subsubsection{Light Leakage into the Luminometer}

To complete this test, the light of the empty luminometer chamber was first measured and incrementally, light measurements of an empty small test tube, $100 \mu \mathrm{L}$ of UltraCheque1 and the small test tube and the mixture of $100 \mu \mathrm{L}$ of Ultrachequel and Luminase were measured as described by Section 5.7.1.1. The UltraCheque1 reagent is a solution of $1 \mathrm{ng}$ $\mathrm{ATP} / \mathrm{mL}$ and in the presence of Luminase is expected to have a significantly higher amount of light measured. 
The average light reading of three replicates showed that:

- The empty chamber had a light reading of $1 \pm 1 \mathrm{RLU}$;

- The empty test tube had a reading of $3 \pm 1 \mathrm{RLU}$;

- The UltraCheque1 reagent added to the test tube had a light reading of $3 \pm 1 \mathrm{RLU}$, and;

- The mixture of UltraChequel and Luminase added to the test tube had a light reading of $12,600 \pm 575 \mathrm{RLU}$, where UltraChequel is a solution containing $1 \mathrm{ng}$ $\mathrm{ATP} / \mathrm{mL}$.

These results show that the amount of light seeping into the luminometer was very low. There is a maximum of $3 \pm 1$ RLU that could be attributed to the test tube. There was no difference in the amount of light recorded between the empty test tube and the UltraCheque1 solution and therefore there was no significant amount light produced from the UltrCheque1 solution. For a solution of $1 \mathrm{ng} \mathrm{ATP} / \mathrm{mL}$ an error of $0.02 \%$ can be attributed to light leakage which is considered negligible.

\subsubsection{Background Levels of LuminUltra Reagents}

In this experiment, the background light levels of the LuminUltra reagents were tested with the purpose of determining the contributions of these reagents to the amount of light measured. To complete the experiment, the modified ATP assay procedure outlined in Section 5.7.1.2 with no sample volume being filtered. Two ATP assays were completed in duplicate: the first with Luminase and the second with XXL Luminase. All other ATP assay reagents used were the same. 
Results of two assays using regular Luminase measured was 7 RLU while the two assays performed with XXL Luminase measured 59 RLU and 47 RLU respectfully. The ATP assays were completed in the absence of a sample volume and quantities of light measured represents the background readings of the respected reagents. The XXL Luminase reagent produced higher RLU values than the Luminase reagent for the same amount of ATP standard solution. The XXL Luminase reagent is thus considered more sensitive. During the study, values of 7 RLU for an ATP test using regular Luminase and 59 RLU for an ATP test using XXL Luminase were below $10 \%$ of the total amount of light measured. For this reason, no correction factor has been applied to the results of this study.

\subsubsection{Background Levels of Deionized Water and Peptone Water}

Deionized water causes disruption of the osmotic pressure of cells and is therefore not suggested for sample dilution in microbiology. The goal of this experiment was to measure and compare the background RLU readings of deionized water and peptone water, and determine whether using peptone water would significantly affect the ATP test results. To complete this experiment, two ATP luminescence assays with Luminase were completed following the procedure described in Section 5.7.1.2. The first assay was completed using $10.0 \mathrm{~mL}$ of deionized water and the second using $10.0 \mathrm{~mL}$ of $0.1 \%$ peptone water. Before testing, both the deionized water and the $0.1 \%$ peptone were autoclaved for 15 minutes at $121^{\circ} \mathrm{C}$ and allowed to cool to room temperature. The average and standard deviation of four replicates are reported. 
The results of the ATP assay showed that:

- Deionized water produced $19 \pm 2 \mathrm{RLU}$ (or approximately $2 \mathrm{RLU} / \mathrm{mL}$ ), and;

- $0.1 \%$ peptone water produced $39 \pm 6 \mathrm{RLU}$ (or approximately $4 \mathrm{RLU} / \mathrm{mL}$ )

While these results show that the background readings are low, they may become significant at lower bacteria concentrations where the amount of ATP in solution is low.

\subsubsection{REPEATABILITY OF THE ATP ASSAY}

The purpose of this experiment was to examine the repeatability of the ATP assay. An initial experimental solution was prepared by adding $50 \mu \mathrm{L}$ of $E$.coli stock solution to 500 $\mathrm{mL}$ of PBS to obtain approximately $10^{5} \mathrm{CFU} / \mathrm{mL}$. Two dilutions of this initial solution were prepared, -2 and $-3 \log$ dilutions, to obtain approximate bacteria concentrations of $10^{3}$ and $10^{2} \mathrm{CFU} / \mathrm{mL}$ respectfully. Then, the 5 replicates of the ATP assay with regular Luminase were completed for each solution with sample volumes of $50.0 \mathrm{~mL}$. The Luminase had an UltraChequel value of 15,140 RLU. The procedures for the ATP assay are outlined in Section 5.5.

The average and standard deviation of the five replicates is presented in Table 6.1 and illustrated in Figure 6.1. Of the E.coli bacteria concentrations examined there seems to be a linear trend such that decreasing the bacteria concentration by a factor of ten results in the reduction of the amount of ATP measured also by a factor of ten. In addition, the results show that there was a very good consistency in the observed ATP quantities as indicated by the low standard deviations, which are one order of magnitude below the value of the average. Consequently, the results of the ATP assay are considered repeatable. 
Table 6.1: Summary of results from repeatability study

\begin{tabular}{|l|r|r|r|r|}
\hline & \multicolumn{2}{|c|}{$\begin{array}{c}\text { Bacteria Concentration } \\
(\mathbf{C F U} / \mathbf{m L})\end{array}$} & \multicolumn{2}{c|}{$\begin{array}{c}\text { Amount of ATP } \\
(\mathbf{p g} / \mathbf{m L})\end{array}$} \\
\hline Sample No. & Average & Standard Deviation & Average & Standard Deviation \\
\hline Sample 1 & $1.4 \mathrm{E}+05$ & $4.0 \mathrm{E}+03$ & 36.2 & 5.9 \\
\hline Sample 2 & $3 \mathrm{E}+03$ & $1 \mathrm{E}+03$ & 1.3 & 0.3 \\
\hline Sample 3 & $1.2 \mathrm{E}+02$ & $1 \mathrm{E}+01$ & 0.33 & 0.07 \\
\hline
\end{tabular}

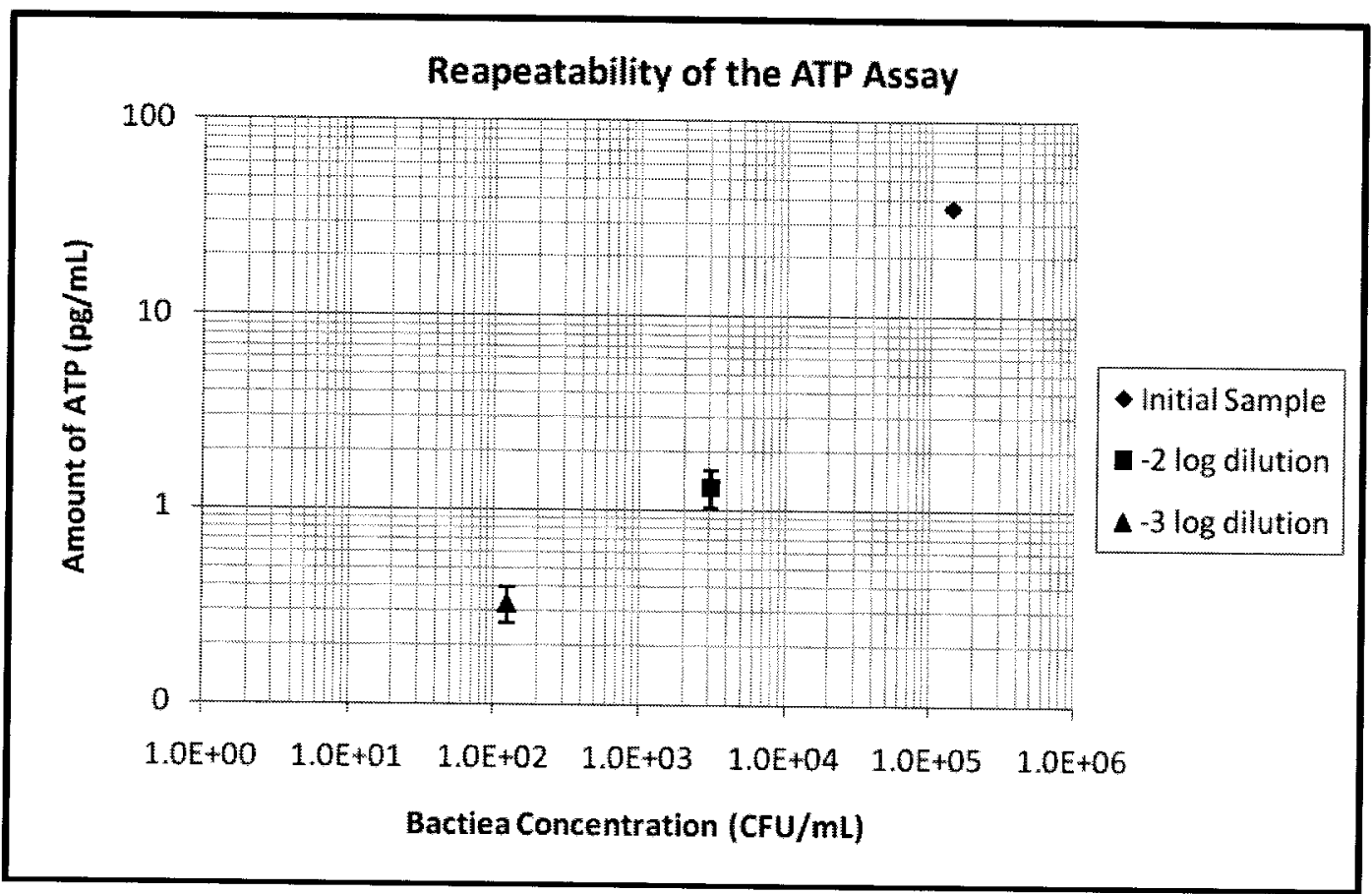

Figure 6.1: Results of the repeatability test of three bacteria concentrations $\left(10^{5}, 10^{3}\right.$ and $10^{2}$ $\mathrm{CFU} / \mathrm{mL}$ ) where the average of five replicates is shown on a log-log scale. 


\subsubsection{EFFECT OF TURBIDITY ON THE ATP ASSAY}

The aim of this experiment was to examine the effect of water turbidity on the results of the ATP assay. A sample of river water from the Rideau River near Carleton University's campus (Ottawa, ON) was collected. The turbidity of the water sample was increased incrementally with a bentonite clay solution. The turbidity of samples as measured (see Section 5.7.1.3) in parallel with the ATP using the ATP assay (see procedure in Section $5.5)$.

The results of this experiment are presented in Figure 6.2, where the average of three turbidity replicates (x-axis) are plotted against each measurement of the amount of ATP. The ATP assays were performed with $15.0 \mathrm{~mL}$ samples, in duplicate, with XXL Luminase with an UltraCheque1 value of 336,522 RLU. Each replicate is represented in the figure.

Surface waters are expected to have a turbidity of less than 35 NTU. The initial turbidity of the river water sample, with no clay solution added, was $2.56 \pm 0.10$ NTU which was incrementally increased to a turbidity of $32.43 \pm 0.55$ NTU.

The average initial amount of ATP was $495 \mathrm{pg} / \mathrm{mL}$, which was also the highest amount of ATP measured. However, the results of a one-way ANOVA determined that the quantities of ATP measured with increasing turbidity were not significantly different from the initial sample with no added turbidity $(p=0.11)$. Therefore, there was no significant effect of increasing the turbidity from $2.56 \pm 0.1 \mathrm{NTU}$ to $32.43 \pm 0.55 \mathrm{NTU}$ on the ATP test results. 
However, as the turbidity of the solution increased, it became more difficult to filter the solution. This is because the clay particles were larger than the pores of the filter paper and clogged the pores of the filter. While the ATP assay has been found suitable for the use of turbid waters, there would be a limit to the turbidity of suitable samples.

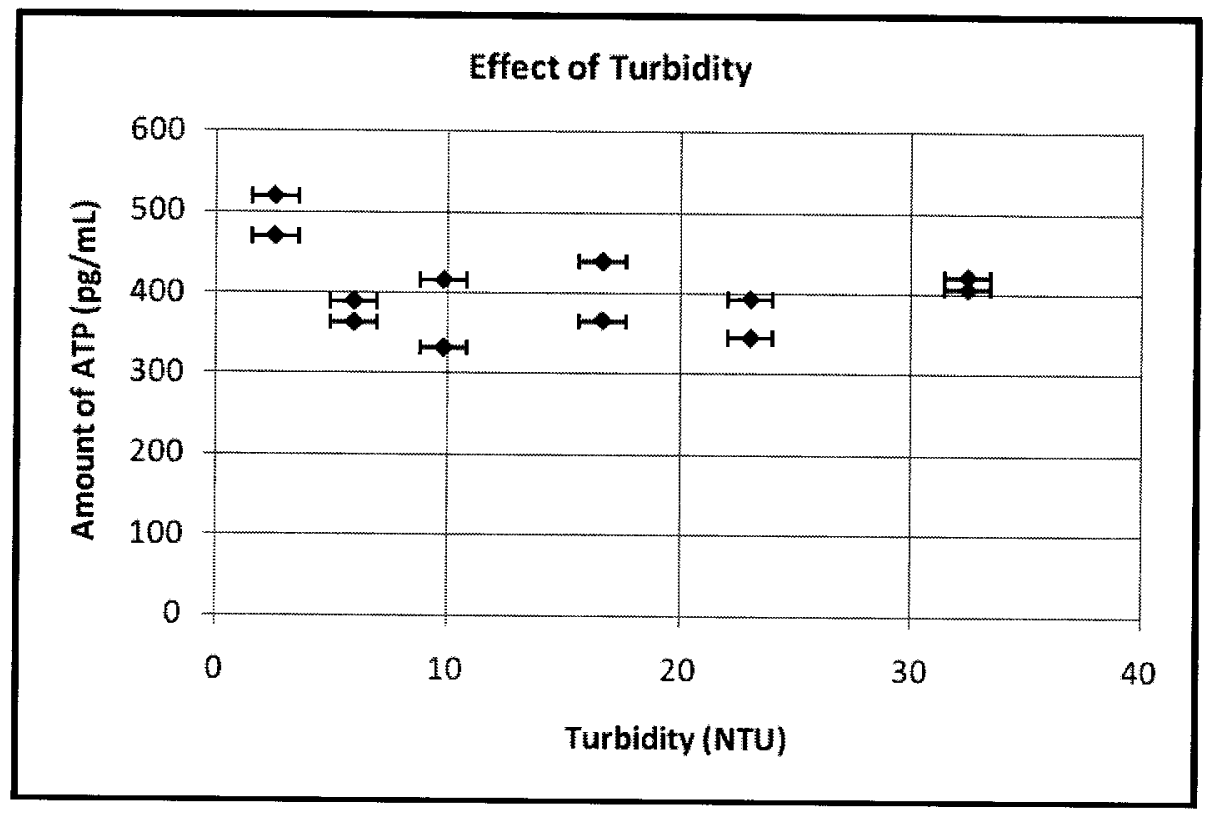

Figure 6.2: Amount of ATP measured from a sample of water from the Rideau River with increasing turbidity. 


\subsubsection{CALIBRATION CURVE WITH 0.1\% PEPTONE}

Calibration curves were created to show the response of the ATP assay to solutions of known bacteria concentrations, as confirmed by membrane filtration.

Four dilutions of an initial sample were examined in parallel with the membrane filtration and the ATP assay, see Section 5.4 and Section 5.5, respectively, for procedural details. The results of each test will first be examined separately, followed by a combined analysis presented in the form of a calibration curve. The initial solution had an E.coli of $1.1 \times 10^{5} \pm 1 \times 10^{4} \mathrm{CFU} / \mathrm{mL}$ (as measured by membrane filtration) and is referred to as the 0 $\log$ dilution sample. Subsequent dilutions will be referred to as their dilution factor. For example, a dilution of the initial sample of 100 , which would be equivalent to approximately $10^{3} \mathrm{CFU} / \mathrm{mL}$ would be referred to as a $-2 \log$ dilution of the initial sample.

The results of the membrane filtration can be seen Figure 6.3, where each data point represents the geometric mean of three replicates and the error bars represent the variance of these replicates. In this instance, the error bars are smaller than the point markers and are difficult to see. For more detailed data please see Appendix A.

As expected, the results of the membrane filtration indicated that increasing the dilution by a factor of $10\left(\mathrm{x}\right.$-axes) resulted in a linear $\left(\mathrm{R}^{2}=1.00\right)$ decrease in the concentration of E.coli by a factor of 10 (y-axes). This linear relationship is represented by the equation:

$$
\boldsymbol{y}=\mathbf{0 . 9 9 x + 5 . 0 4}
$$

where $x$ is the $\log$ dilution of the initial sample and $y$ is the $\log$ of the bacteria concentration. A blank sample of only $0.1 \%$ peptone water was performed and no colonies were observed giving E.coli bacteria concentration of $0 \mathrm{CFU} / \mathrm{mL}$. 
It is important to note that initially a total of five solutions were prepared (log dilutions of $0,-2,-3,-4$ and -5$)$ for testing but the $-4 \log$ dilution of the initial sample was accidently omitted. Based on the aforementioned equation of the line (see Equation 6.1), the missing bacteria concentration can be calculated to be $1.2 \times 10^{1} \mathrm{CFU} / \mathrm{mL}$.

The ATP assay was performed with a sample size of $10.0 \mathrm{~mL}$ and regular strength Luminase with an UltraChequel value of 13,295 RLU. A blank was performed with $0.1 \%$ peptone water and the amount of ATP measured was $1.4 \pm 0.04 \mathrm{pg} / \mathrm{mL}$. The results of the ATP assay are presented in Figure 6.4 where the log dilution of the initial sample (x-axis) is plotted against the measured amount of ATP (y-axis).

One would expect to see a decrease in the amount of ATP proportional to the increase in dilution of the initial sample. However, the results shown in Figure 6.4 show a lack of linear relationship $\left(\mathrm{R}^{2}=0.06\right)$ between the amount of ATP and the logarithm of the dilution of the initial sample. Examining the results on a $\log -\log$ scale, see Figure 6.5 , does not offer any improvements to the results $\left(\mathrm{R}^{2}=0.09\right)$. Upon closer inspection, with the exceptions of the $-2 \log$ dilution of the initial sample and the blank sample, the measured quantities of ATP all have a magnitude of $10^{2} \mathrm{pg} \mathrm{ATP} / \mathrm{mL}$ despite the different dilutions of the initial sample.

To investigate the significance of the data, a one-way analysis of variance (ANOVA) was performed and concluded that there was a significant difference in the sample means $(p<$ 0.001). To further investigate, Dunnett's test, which compares each sample set to that of the blank, was performed and concluded that all sample-sets differed from the blank. However, the Tukey HSD test, which compares pairs of data to determine if they are 
significantly different from each other, determined that, as suspected, the $0,-3$ and $-4 \log$ dilutions were not significantly different from each other and the 0 and $-5 \log$ were not significantly different from each other. The only data point remaining that was significantly different from the others was the $-2 \log$ dilution $(p<0.001)$. For a full account of the statistical analysis, please see Appendix A.

The resulting calibration curve can be seen in Figure 6.6. The lack of linearity of the results of the ATP assay was surprising and necessitated further testing. The lack of difference between the $0,-3$ and $-4 \log$ dilutions was also surprising indicating the lower sensitivity limit of the test to be in the range of $10^{5} \mathrm{CFU} / \mathrm{mL}$. In these experiments, peptone water was used as a diluent, which was suggested by the manufacturer of the ATP test. However, the spike observed in measured ATP at the -2 dilution may point to a possible regrowth in samples during the testing period. The subsequent chapter will aim to retest and improve the performance and sensitivity of the ATP test. 


\section{Membrane Filtration}

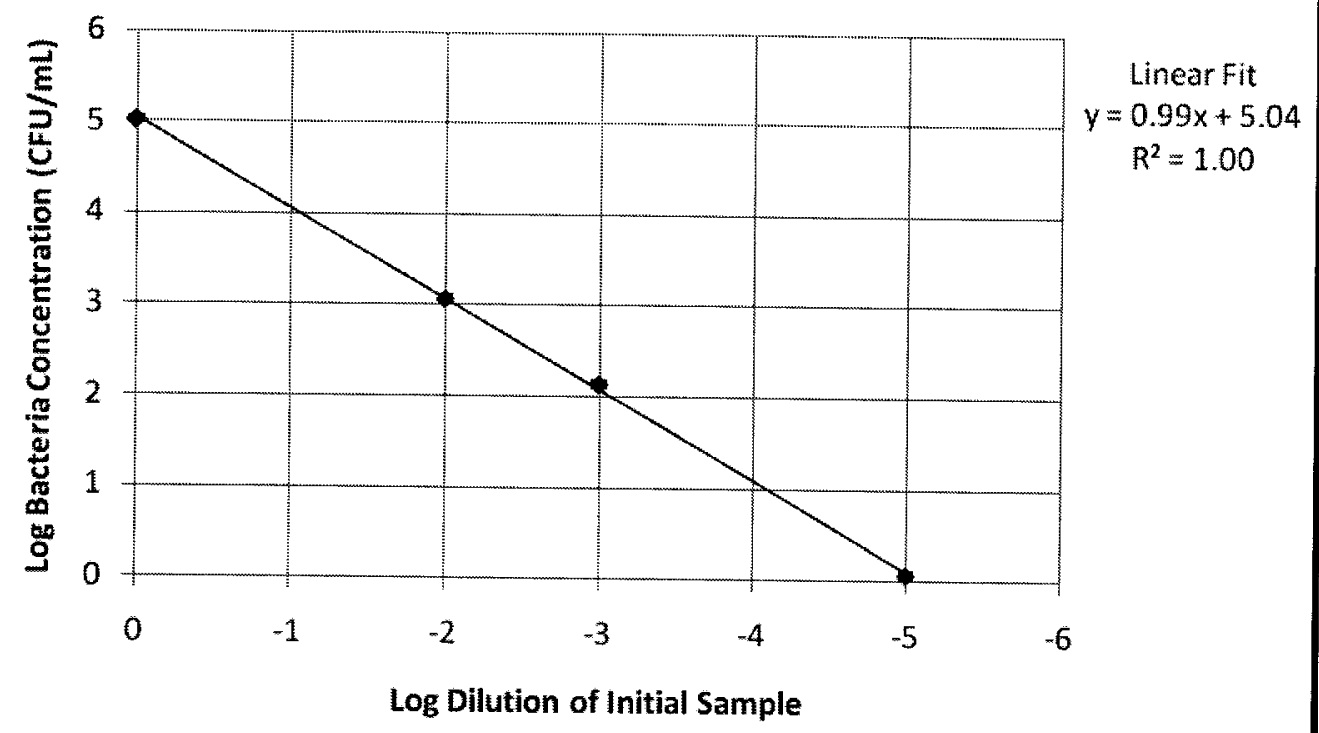

Figure 6.3: Results of the membrane filtration showing the logarithm bacteria concentration of the logarithm of four dilutions of a sample with initial bacteria concentration of $1.1 \times 10^{5} \pm 1 \times 10^{4} \mathrm{CFU} / \mathrm{mL}$ (as measured by membrane filtration) with $0.1 \%$ peptone water.

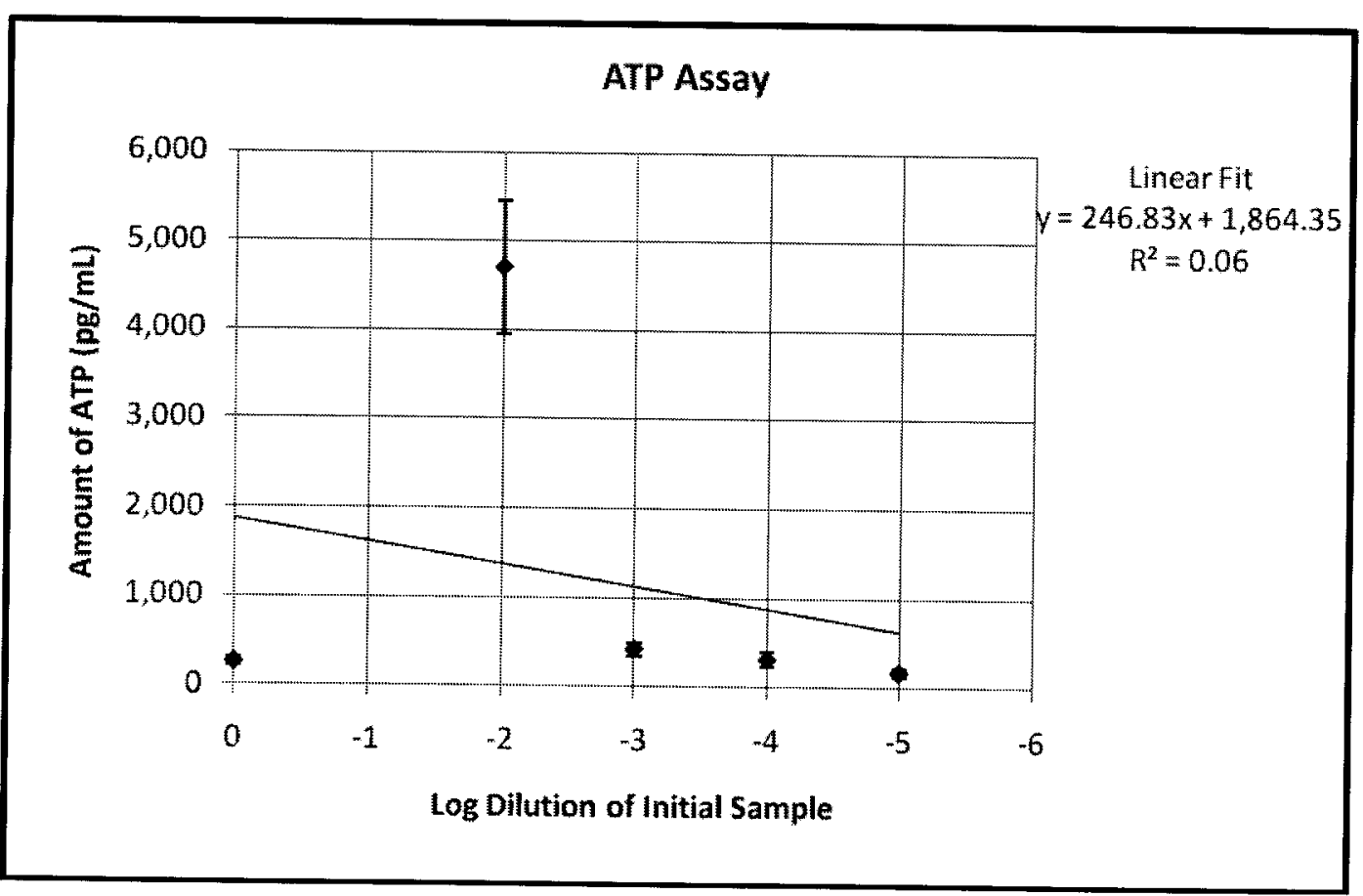

Figure 6.4: Results of the ATP assay with Luminase showing the amount of ATP of the logarithm of five dilutions of a sample with initial bacteria concentration of $1.1 \times 10^{5} \pm 1 \times 10^{4} \mathrm{CFU} / \mathrm{mL}$ (as measured by membrane filtration) with $0.1 \%$ peptone water and $10.0 \mathrm{~mL}$ sample volumes. 


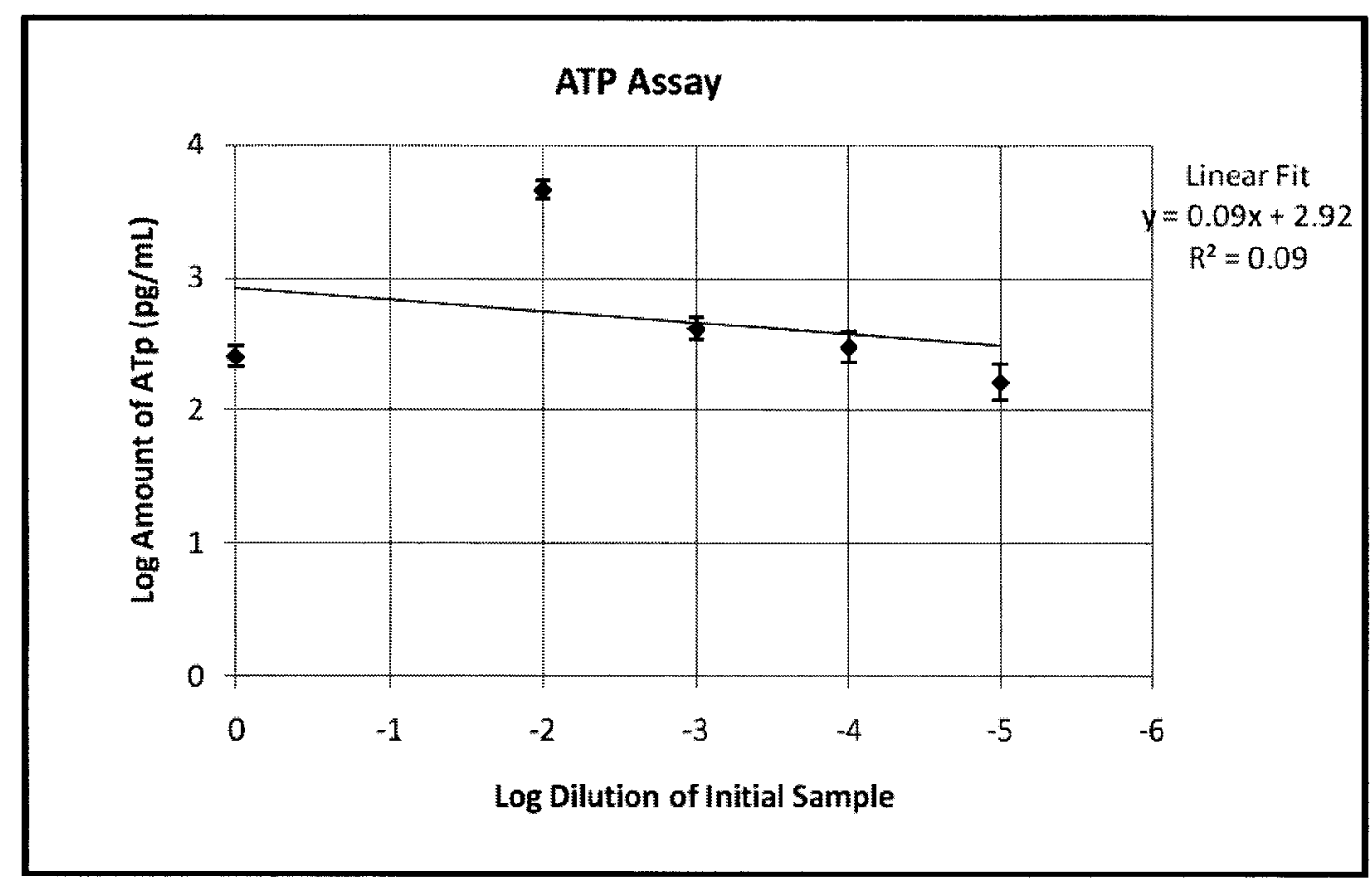

Figure 6.5: Results of the ATP assay with Luminase showing the logarithm of the amount of ATP of the logarithm of five dilutions of a sample with initial bacteria concentration of $1.1 \times 10^{5} \pm$ $1 \times 10^{4} \mathrm{CFU} / \mathrm{mL}$ (as measured by membrane filtration) with $0.1 \%$ peptone water and $10.0 \mathrm{~mL}$ sample volumes.

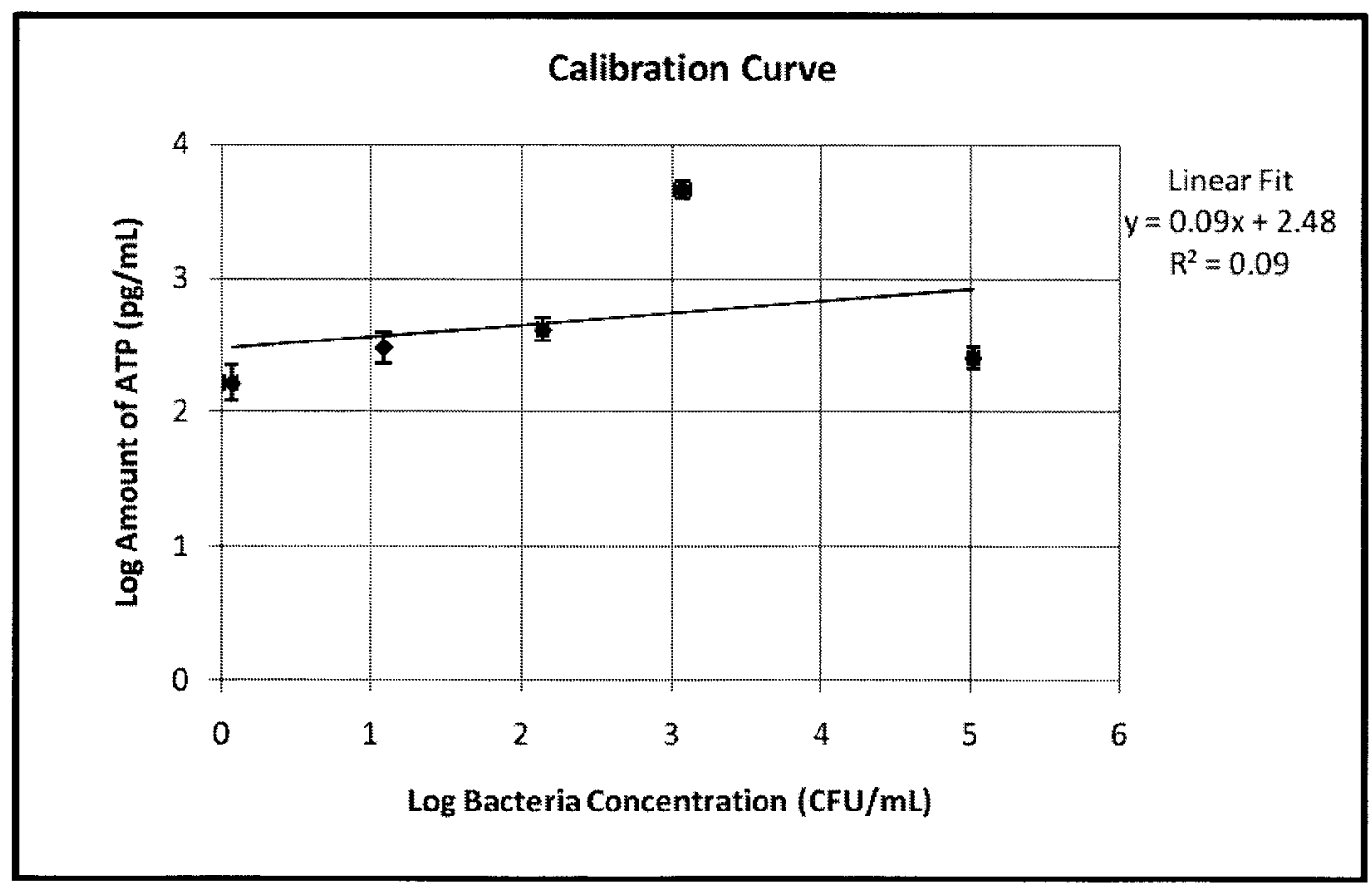

Figure 6.6: Calibration curve between membrane filtration and ATP assay with Luminase using $10.0 \mathrm{~mL}$ samples and $0.1 \%$ peptone. 


\subsubsection{CALIBRATION CURVE WITH PBS}

Similar to the previous analysis, the purpose of this calibration curve was to compare the response of the ATP assay and the membrane filtration to solutions of known bacteria concentrations. In this calibration curve, the peptone water has been replaced with PBS. The ATP assay and the membrane filtration were performed in parallel with a total of four dilutions of an initial solution of $1.2 \times 10^{5} \pm 3.1 \times 10^{4} \mathrm{CFU} / \mathrm{mL}$ (confirmed by membrane filtration). The $\log$ dilutions of the initial solution examined were: $0,-2,-4$ and -6 .

The results of the membrane filtration, presented in Figure 6.7, show that, as expected, the dilution of the initial sample solutions increased by a factor of 10 (x-axes) that the concentration of E.coli (y-axes) decreases linearly by a factor of $10\left(\mathrm{R}^{2}=1.00\right)$. The equation of the line of best fit is:

$$
y=1.06 x+5.09(6.2)
$$

where $x$ is the log dilution of the initial sample and $y$ is the $\log$ of the E.coli bacteria. The data points of Figure 6.7 represent the geometric mean of three replicates and the error bars their respected variances. A blank analysis using PBS (with no bacteria) was also performed and produced three replicates with no colony forming units.

It is important to note that in this experiment, the $-6 \log$ dilution corresponds to a bacteria concentration of $5 \times 10^{-2} \pm 2 \times 10^{-2} \mathrm{CFU} / \mathrm{mL}$ which is equivalent to $5 \times 10^{0} \pm 2 \times 10^{0} \mathrm{CFU} / 100$ $\mathrm{mL}$. The microbial quality of purified drinking water is typically reported in CFU/100 $\mathrm{mL}$. 
The ATP assay was performed with sample volumes of $10.0 \mathrm{~mL}$ and Luminase with an UltraCheque1 of 11,610 RLU. Two replicates were performed with pure PBS and produced 0.5 and $0.6 \mathrm{pg} \mathrm{ATP} / \mathrm{mL}$, respectively. The results of the ATP assay are presented in Figure 6.8. A linear trend, similar to that observed during the membrane filtration (see Figure 6.7), was expected and to a certain extent was observed. There was a decrease in the logarithm of the amount of ATP measured as the logarithm of the dilution of the initial sample increased however, with an $\mathrm{R}^{2}$ value of 0.79 it is less than ideal. The calibration curve, presented in Figure 6.9 , also shows low-linearity with a $\mathrm{R}^{2}$ value of 0.79. When the exponential fit was applied to the latter two figures the $R^{2}$ values improved to 0.89 which may suggest that the results of the ATP assay are more closely modeled by an exponential curve. However, more investigation is required to confirm this hypothesis.

To further investigate the significance of the results of the ATP assay, a one-way ANOVA was performed and identified that there was a significant difference between the means of the samples points $(p<0.0001)$. However, the Dunnett's test showed that the only data point that was significantly different from the PBS was the initial sample solution (with no dilution) $\left(p=1.19 \times 10^{-5}\right)$, for a full report of the statistical analysis, see Appendix A. This suggests that the sensitivity limit of the test is approximately $10^{5}$ $\mathrm{CFU} / \mathrm{mL}$, which is the same conclusion reached in the previous section, Section 6.1.4. With bacteria concentrations less than $10^{4} \mathrm{CFU} / \mathrm{mL}$ there does not seem to be enough ATP being collected to produce a significant amount of light. The subsequent chapter will aim to improve the sensitivity limit of the ATP assay. 
Membrane Filtration

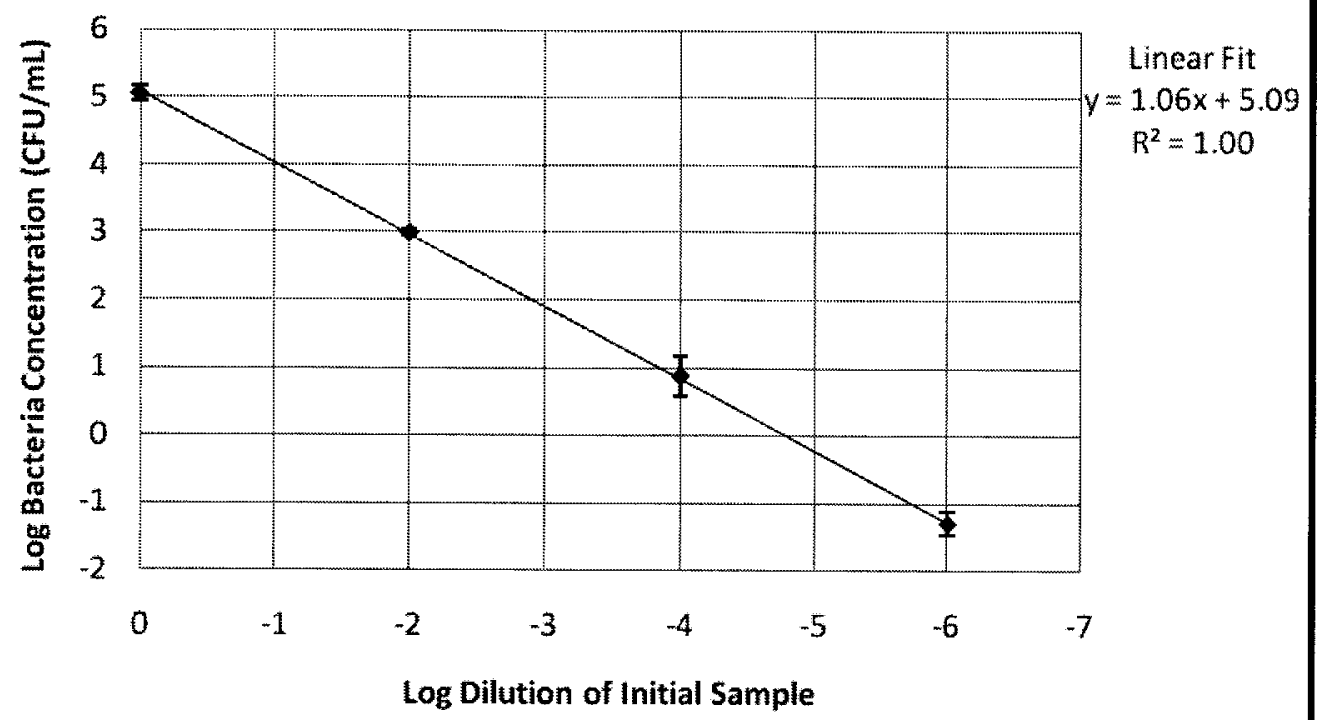

Figure 6.7: Results of the membrane filtration showing the logarithm bacteria concentration of the logarithm of four serial dilutions of a sample with initial bacteria concentration of $1.2 \times 10^{5} \pm$ $3 \times 10^{4} \mathrm{CFU} / \mathrm{mL}$ (as measured by membrane filtration) with PBS.

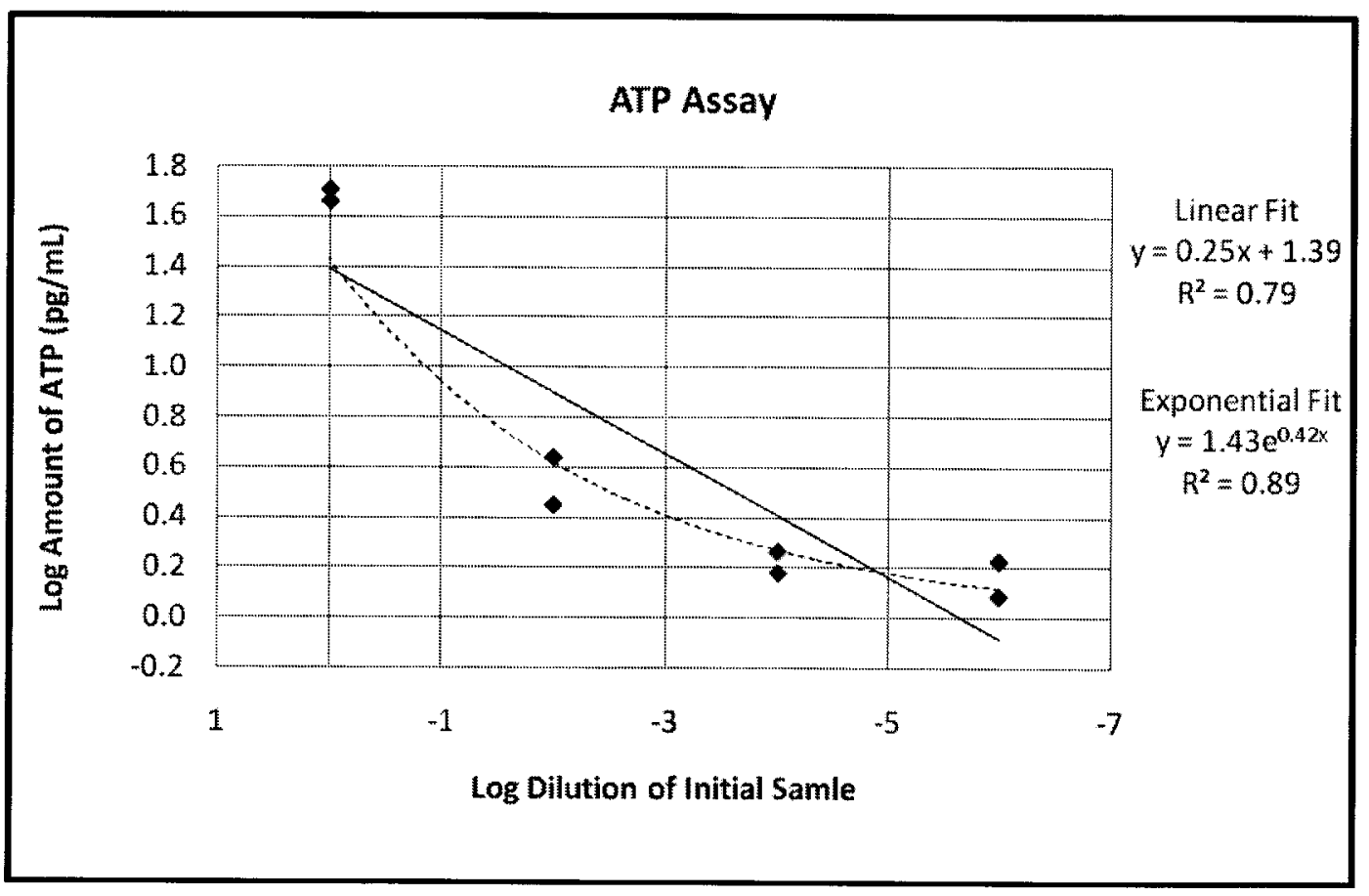

Figure 6.8: Results of the ATP assay with Luminase showing the logarithm of the amount of ATP of the logarithm of four dilutions of a sample with initial bacteria concentration of $1.2 \times 10^{5} \pm$ $3 \times 10^{4} \mathrm{CFU} / \mathrm{mL}$ (as measured by membrane filtration) with PBS and $10.0 \mathrm{~mL}$ sample volumes. 


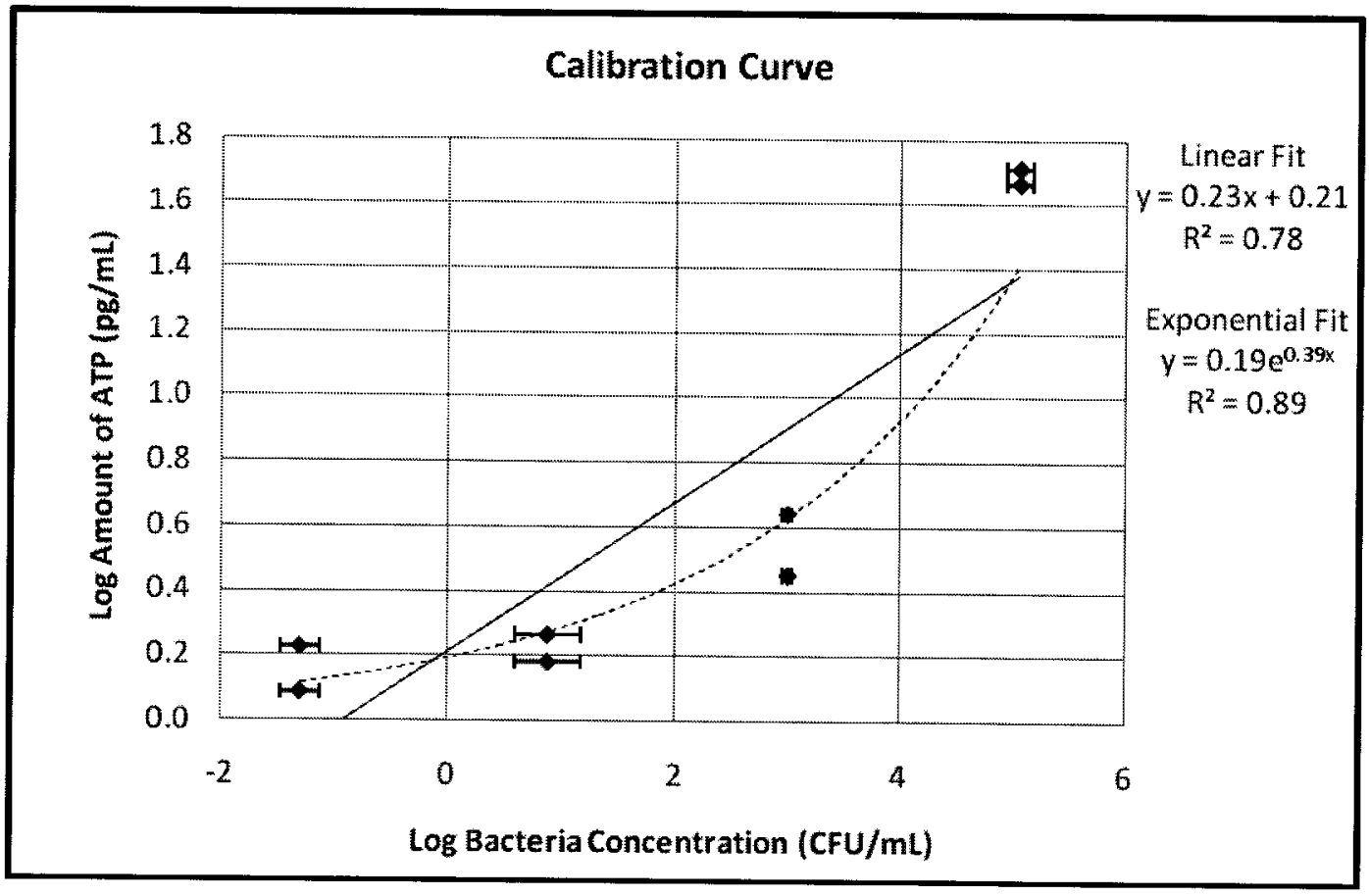

Figure 6.9: Calibration curve between membrane filtration and ATP assay using $10.0 \mathrm{~mL}$ samples and PBS.

\subsubsection{CALIbration CURVE For High BACteria ConCENTRATIONS}

The previous calibration curves indicated that the sensitivity limit of the ATP assay may be in the order of $10^{5} \mathrm{CFU} / \mathrm{mL}$. The purpose of this calibration curve was to examine the behaviour of the ATP assay at higher bacteria concentrations. It is possible that the ATP assay has a linear relationship for high bacteria counts, but linearity is lost at lower bacteria concentrations possibly due to the limitation in the sensitivity of the test. The purpose of this calibration curve was to determine the upper limits of the ATP assay and to determine if the calibration curve would be linear at higher bacteria concentrations. For the purpose of this study, a high bacteria concentration is defined as bacteria concentrations above $10^{5} \mathrm{CFU} / \mathrm{mL}$ (as measured by membrane filtration). The bacteria concentration of the non-diluted E.coli stock solution is approximately $10^{9} \mathrm{CFU} / \mathrm{mL}$, which is the upper limit of the high bacteria concentrations range. For a description of the 
procedure followed to complete the calibration curve presented in this section, see Section 5.7.1.4.

Membrane filtration was used to measure the bacteria concentration of the $10^{5} \mathrm{CFU} / \mathrm{mL}$ solution, which was found to be $1.5 \times 10^{5} \pm 3 \times 10^{4} \mathrm{CFU} / \mathrm{mL}$. Due to the high level of linearity of the membrane filtration technique, as demonstrated in Figure $6.3\left(\mathrm{R}^{2}=1.00\right)$ and Figure $6.7\left(\mathrm{R}^{2}=1.00\right)$, other bacteria concentrations were estimated with Equation 6.2 .

The ATP assay was performed beginning with the solution with a bacteria concentration of $1.5 \times 10^{5} \pm 3 \times 10^{4} \mathrm{CFU} / \mathrm{mL}$ and proceeding with the higher concentrated solutions. When possible, sample volumes of $50.0 \mathrm{~mL}$ was used. However, the limit of the luminometer was reached with solutions higher than $10^{8} \mathrm{CFU} / \mathrm{mL}$ and the sample volume was reduced to $5.0 \mathrm{~mL}$ and further reduced to $1.0 \mathrm{~mL}$ if required. The Luminase had an UltraChequel value of $11,833 \pm 400$ RLU. The ATP assay was performed in duplicate. The resulting calibration curve for the quantity of ATP measured for each bacteria concentration examined is presented in Figure 6.10, where each data point represents one replicate of the ATP assay.

At higher bacteria concentrations the logarithm of the amount of ATP is linearly dependant to the logarithm of the bacteria concentration $\left(R^{2}=0.98\right)$. The equation of the line of best fit is:

$$
y=0.87 x-2.26 \text { for } 5<x<9\}
$$


where $x$ is the logarithm of the amount of ATP (pg/mL) and $y$ is the logarithm of the E.coli bacteria $(\mathrm{CFU} / \mathrm{mL})$. These results confirm that the ATP results are linear with bacteria counts when the samples contain high levels of bacteria (greater $10^{5} \mathrm{CFU} / \mathrm{mL}$ ), and the test has limitations for samples that have low bacteria counts or may not be linear for samples with low bacteria counts.

It is important to note the volume filtered for the ATP assay was increased to $50.0 \mathrm{~mL}$, where previous calibration curves used sample volumes of $10 \mathrm{~mL}$, and the sample volume was only decreased if the sensitivity limits of the luminometer were reached. This may suggest that increasing the sample volumes may offer significant improvements to the performance of the ATP assay at lower bacteria concentrations.

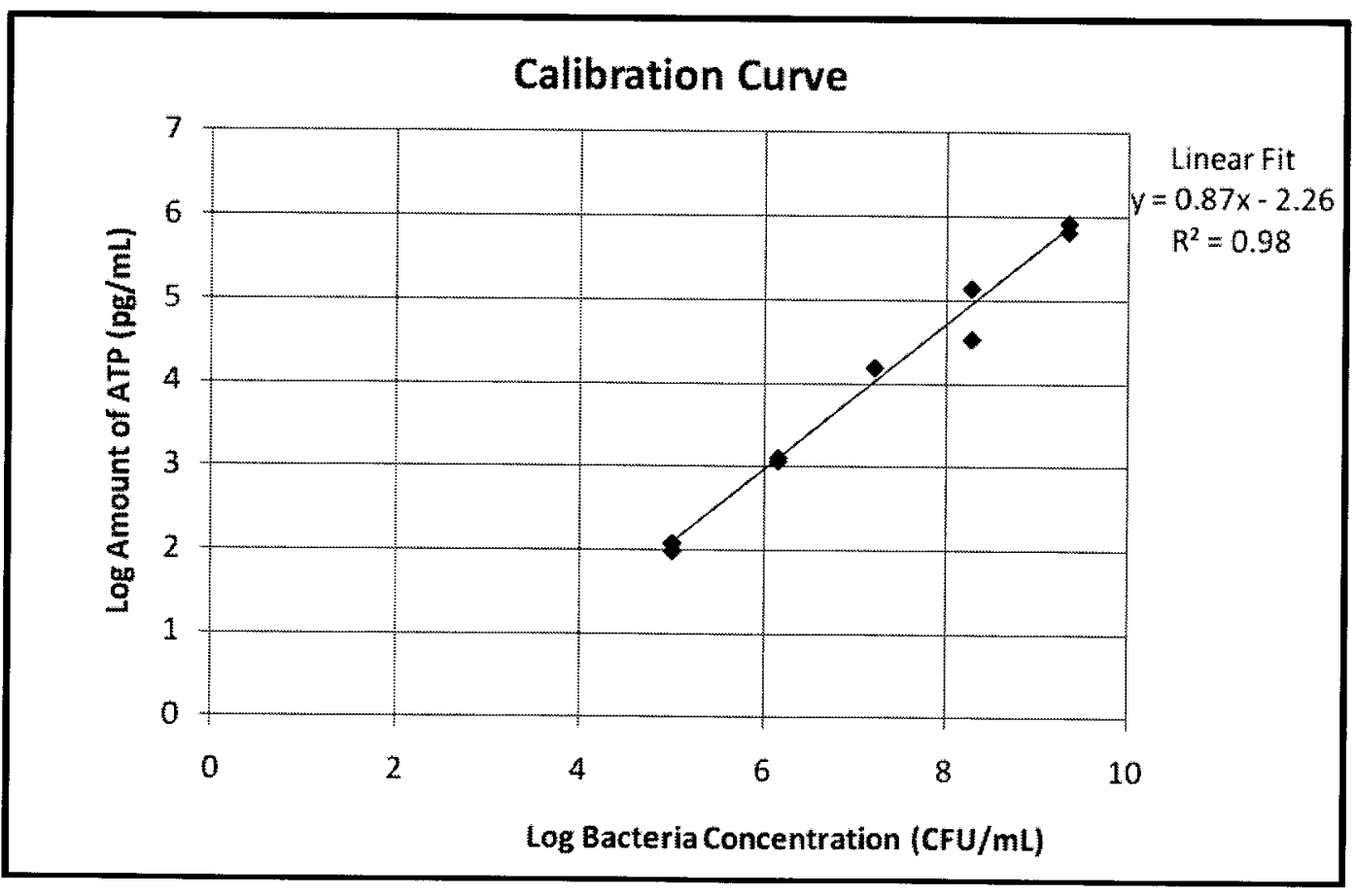

Figure 6.10: Calibration curve between membrane filtration and ATP assay using solutions with high bacteria concentrations $\left(10^{5}\right.$ to $\left.10^{9} \mathrm{CFU} / \mathrm{mL}\right)$ 


\subsection{Phase I: Optimization of The ATP Assay}

After preliminary testing, it was evident that the sensitivity of the ATP assay needed improvement. From the preliminary testing, the sensitivity limit appeared to be approximately $10^{5} \mathrm{CFU} / \mathrm{mL}$.

The purpose of these experiments was to improve the sensitivity and accuracy of the ATP luminescence assay by changing key elements of its protocol.

\subsubsection{EFFECT OF VARYING THE PORE SIZE OF THE FILTER}

The purpose of this experiment is to compare two sets of ATP assays each using a different filter pore size: $0.7 \mu \mathrm{m}$ and $0.2 \mu \mathrm{m}$. For each set of ATP assays, two sample solutions were tested. The first sample, called Sample 1, consisted $50 \mu \mathrm{L}$ of E.coli stock solution in $500.0 \mathrm{~mL}$ of PBS. The second sample was pure PBS with no added E.coli. Membrane filtration was used to determine that Sample 1 contained a bacteria population of $7.5 \times 10^{5} \pm 1.2 \times 10^{4} \mathrm{CFU} / \mathrm{mL}$ and no E.coli was found in the PBS. The results of this experiment are summarized in Table 6.2 and are presented more in detail in Appendix A.

Similar to membrane filtration, which uses a filter to retain the bacteria population of a sample, the ATP luminescence assay also uses a filter to capture microorganisms present in samples. While membrane filtration uses a $0.45 \mu \mathrm{m}$ pore size filter, the ATP assay uses a filter of $0.7 \mu \mathrm{m} .0 .2-0.22 \mu \mathrm{m}$ pore size filters can filter out (or collect) all of the bacteria present in a sample. Bacteria are single-cell organisms that range in size from $0.2 \mu \mathrm{m}$ to

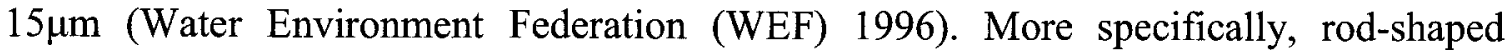
bacteria such as E.coli typically has a diameter between 0.6 to $1.2 \mu \mathrm{m}$ and measures 1.5 to $3.0 \mu \mathrm{m}$ long (Metcalf \& Eddie 2003). While a $0.7 \mu \mathrm{m}$ pore size filter is adequate to capture the E.coli population of a sample, it was suspected that by decreasing the pore 
size, the amount of bacteria cells captured would also increase and consequently increase the accuracy and reproducibility of results as well as increasing the lower detection limit.

It was suspected that decreasing the pore size of the filter, from $0.7 \mu \mathrm{m}$ to $0.2 \mu \mathrm{m}$, would increase the amount of bacteria cells captured and consequently increase the amount of ATP (or light) measured in the sample. As seen in Table 6.2, this trend was observed for both samples, Sample 1 and the PBS.

To further investigate the significance of the increases in ATP a t-test using a two-sample two-tailed analysis was performed. The p-values obtained from this analysis are also shown in Table 6.2 and are compared to a significance level of 0.05. For Sample 1, the ttest concluded that the change in the filter size did not produce any significant difference in the amount of ATP measured $(p=0.046)$. The opposite was true for the PBS sample ( $p$ $=0.046$ ) .

The PBS solution was free of E.coli bacteria and the increase in the quantity of ATP could be attributed background levels, from contamination introduced during filtration or from experimental error. To capture as many microorganisms as possible, a $0.2 \mu \mathrm{m}$ pore size filter would be ideal, however, it is arduous and time-consuming to filter a sample through a $0.2 \mu \mathrm{m}$ pore size filter This makes it difficult to filter any meaningful sample volumes and renders the ATP assay impractical for the use for field experiments without the aid of additional equipment (such as a syringe pump). Changing the filter to $0.2 \mu \mathrm{m}$ would as a result increase the complexity of the test and this idea was not entertained further. 
Table 6.2: Average amount of ATP measured using the ATP luminescence assay comparing $0.7 \mu \mathrm{m}$ and $0.2 \mu \mathrm{m}$ pore size filters

\begin{tabular}{|l|l|r|r|}
\hline & $\begin{array}{l}\text { Average Amount of ATP } \\
\text { (pg ATP/mL) }\end{array}$ & Standard Deviation & $p$-value \\
\hline Sample 1 and $0.7 \mu \mathrm{m}$ filter & 74.06 & 10.30 & \\
\cline { 1 - 3 } Sample 1 and $0.2 \mu \mathrm{m}$ filter & 81.2 & 10.8 & 0.46 \\
\hline PBS and $0.7 \mu \mathrm{m}$ filter & 0.2 & 0.06 & \\
\cline { 1 - 4 } PBS and $0.2 \mu \mathrm{m}$ filter & 2.7 & 1.5 & 0.046 \\
\hline
\end{tabular}

\subsubsection{EFFECT OF VARYING THE AMOUNT OF LYSING SOLUTION}

It was hypothesized that $1 \mathrm{~mL}$ of lysing solution may not be sufficient to release all of the potential ATP from the captured bacteria cells. In addition, due to the very short reaction time with the lysing solution, extraction efficiency might differ from sample to sample leading to variability in ATP results. Thus, increasing the amount of lysing solution may increase the efficiency of the extraction step and also increase the amount of ATP captured. The makeup of the lysing solution is protected by the manufacturer of the ATP assay and therefore was unknown.

Three ATP assays were performed each increasing the amount of lysing solution from 1 $\mathrm{mL}$ to $2 \mathrm{~mL}$ and $3 \mathrm{~mL}$. In addition, $\mathrm{RLU}$ measurements were taken immediately after the test protocol was completed and after approximately 5 minutes after introducing the lysing solution. The results are represented in Figure 6.11 and a more detailed account can be found in Appendix A.

As shown in Figure 6.11, an increase in the amount of lysing solution added decreased the amount of ATP measured in all cases. In addition, allowing more time for the lysing 
solution to take effect appeared to decrease the amount of light produced. In theory, the lysing solution dissolves the cell wall to release the ATP contained within. Further analysis of the lysing solution showed that it was a very strong basic solution with a $\mathrm{pH}$ of 11 . At these high $\mathrm{pH}$ values, the extracted ATP would be destroyed very quickly resulting in a decrease in the ATP quantities measured. Therefore, neither increasing the quantity of the lysing solution nor extending the reaction time would improve the test performance. Thus, $1 \mathrm{~mL}$ of lysing solution measured immediately produced the greatest amount of light.

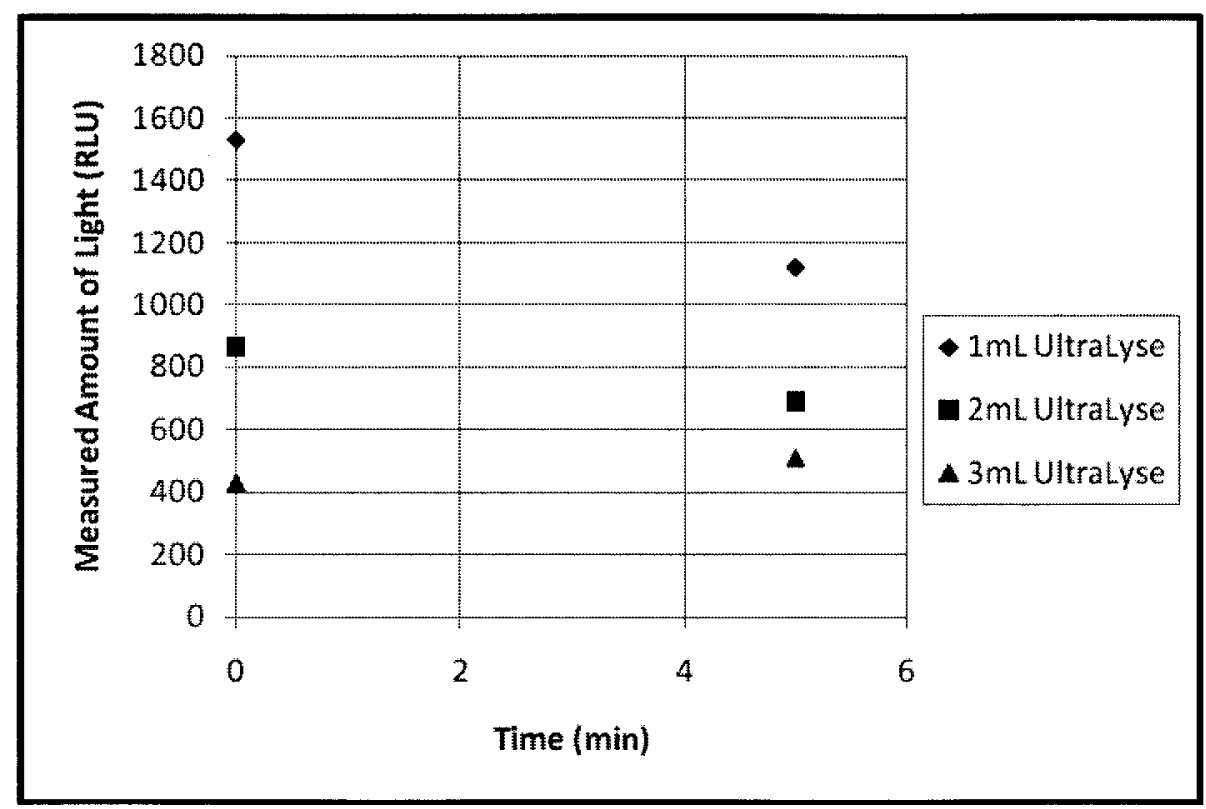

Figure 6.11: Varying the amount of lysing solution and effects of time 


\subsubsection{CALIBRATION CURVE FOR LOW BACTERIA CONCENTRATIONS WITH XXL LUMINASE}

The two initial calibration curves for low bacteria counts $\left(<10^{5} \mathrm{CFU} / \mathrm{mL}\right)$ formulated in Section 6.1 (see Figure 6.9 and Figure 6.10) indicated that the advertized lower limits of the ATP assay were not accurate and a more realistic estimation would be around $10^{5}$ $\mathrm{CFU} / \mathrm{mL}$. To increase the detection limit of the ATP assay, the following experiments were performed using larger sample volumes and the more potent XXL Luminase. After the preliminary phase, it became clear that one of the limitations of the ATP test is related to the amount of light that can be detected by the luminometer. For samples with low bacteria counts, even if the reaction with ATP test reagents is complete, the amount of emitted light might be so small that it may not be picked up by the luminometer. Increasing the sample volume would increase the amount of ATP, thus the amount of light emitted from the sample, which then can be corrected for sample volume after the measurement. Using the more potent XXL Luminase would also increase the detection of present ATP in the sample.

In Section 6.1, a linear relationship was determined between high bacteria concentrations ( $>10^{5}$ and $<10^{9} \mathrm{CFU} / \mathrm{mL}$ ) and the measured quantities of ATP, see Figure 6.10 and the corresponding equation of the line Equation 6.3. Ideally, the calibration curve for low concentrations of bacteria would follow same line and will thus be used to predict the results of the of the ATP assay. 
The first calibration curve presented here compares the results of the ATP assay in response to different bacteria concentrations (confirmed by membrane filtration) using regular strength Luminase and high strength XXL Luminase with sample volumes ranging from $400 \mathrm{~mL}$ to $1,000 \mathrm{~mL}$.

The membrane filtration, presented in Figure 6.12, was performed with three sample dilutions: $10^{5}, 10^{3}$ and $10^{1} \mathrm{CFU} / \mathrm{mL}$. The data points of the figure represent the average of three replicates and the error bars the variances. The other dilution concentrations were estimated with the resulting equation of the line of best fit of Figure $6.16\left(\mathrm{R}^{2}=1.00\right)$ :

$$
\boldsymbol{y}=0.99 x+4.99
$$

where $x$ is the logarithm of the dilution of the initial sample and $y$ is the logarithm of the E.coli bacteria concentration.

The resulting calibration curve, Figure 6.13, was created with two series of ATP assays. The first used regular strength Luminase with an UltraCheque1 value of 9,185 RLU. The second was performed with XXL Luminase with an UltraChequel value of the 443,385 RLU. All solution concentrations were tried a minimum of two times for each type of Luminase. However, if two replicates differed by one order of magnitude, additional replicates were performed to a maximum of four replicates.

Comparing the expected values of the calibration curve to the measured values, it appears that the measured values begin to deviate from the expected curve in between $10^{3}$ and $10^{2}$ $\mathrm{CFU} / \mathrm{mL}$ for both Luminase and XXL Luminase. This range represents the range of the sensitivity limit of the test achieved using larger volumes and is a major improvement from $10^{5} \mathrm{CFU} / \mathrm{mL}$. It also appears that as the concentration of bacteria decreases, the 
precision of the results also decrease. As seen in Figure 6.13, there was increased variability of the data points at lower bacteria concentrations. To complete this calibration curve, sample volumes were increased and ranged between 400 and 1,000 mL. However, the capacity of the syringed used for filtration remained the same. The increased variability may be due to the number of filtrations required for filter the larger sample volumes in increments of $50 \mathrm{~mL}$.

Comparing the amounts of light measured for a same sample, the values measured using XXL are higher than those of the regular Luminase. However, once converted to the amount of ATP in solution, the values are about the same. XXL Luminase was able to produce a stronger light reaction but the amount of light recorded was normalized by the larger UltraCheque1 value.

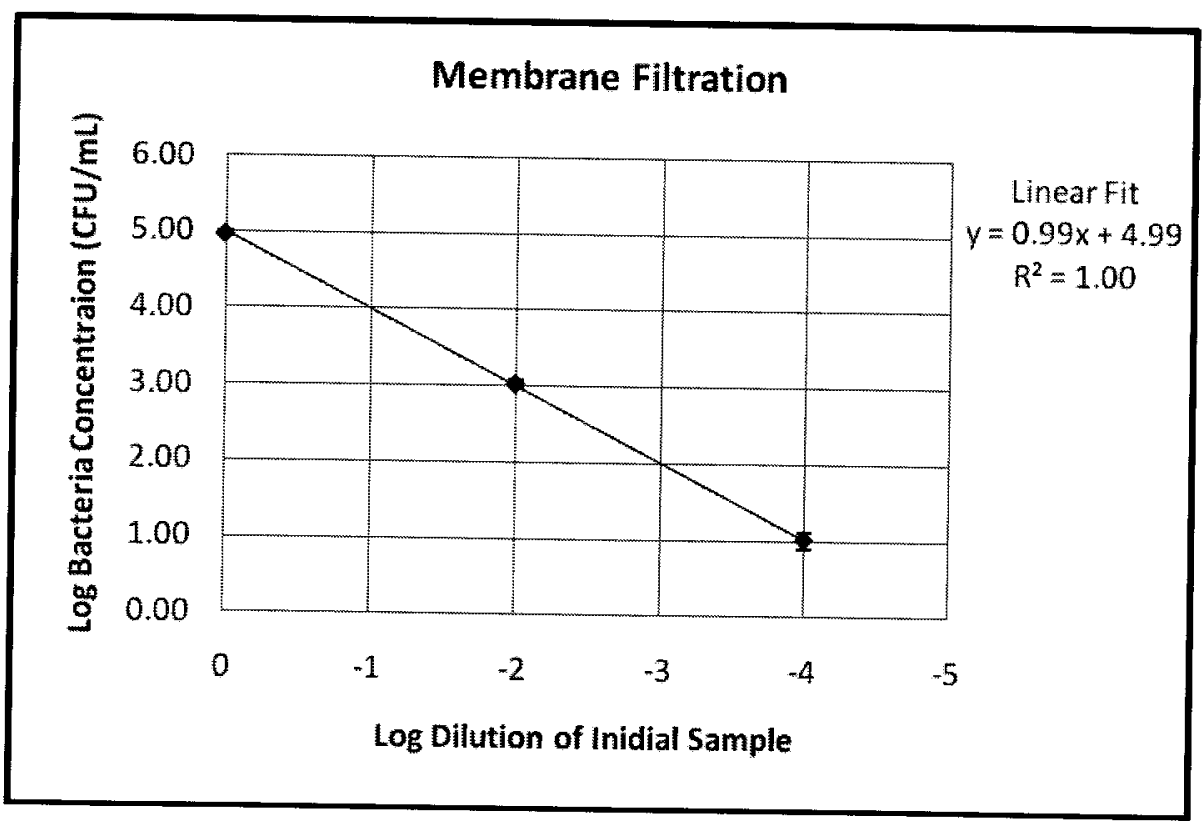

Figure 6.12: Results of the membrane filtration showing the logarithm bacteria concentration of the logarithm of three dilutions of a sample with initial bacteria concentration of $9.5 \times 10^{4} \pm 8 \times 10^{3} \mathrm{CFU} / \mathrm{mL}$ (as measured by membrane filtration) with PBS and low bacteria concentrations. 


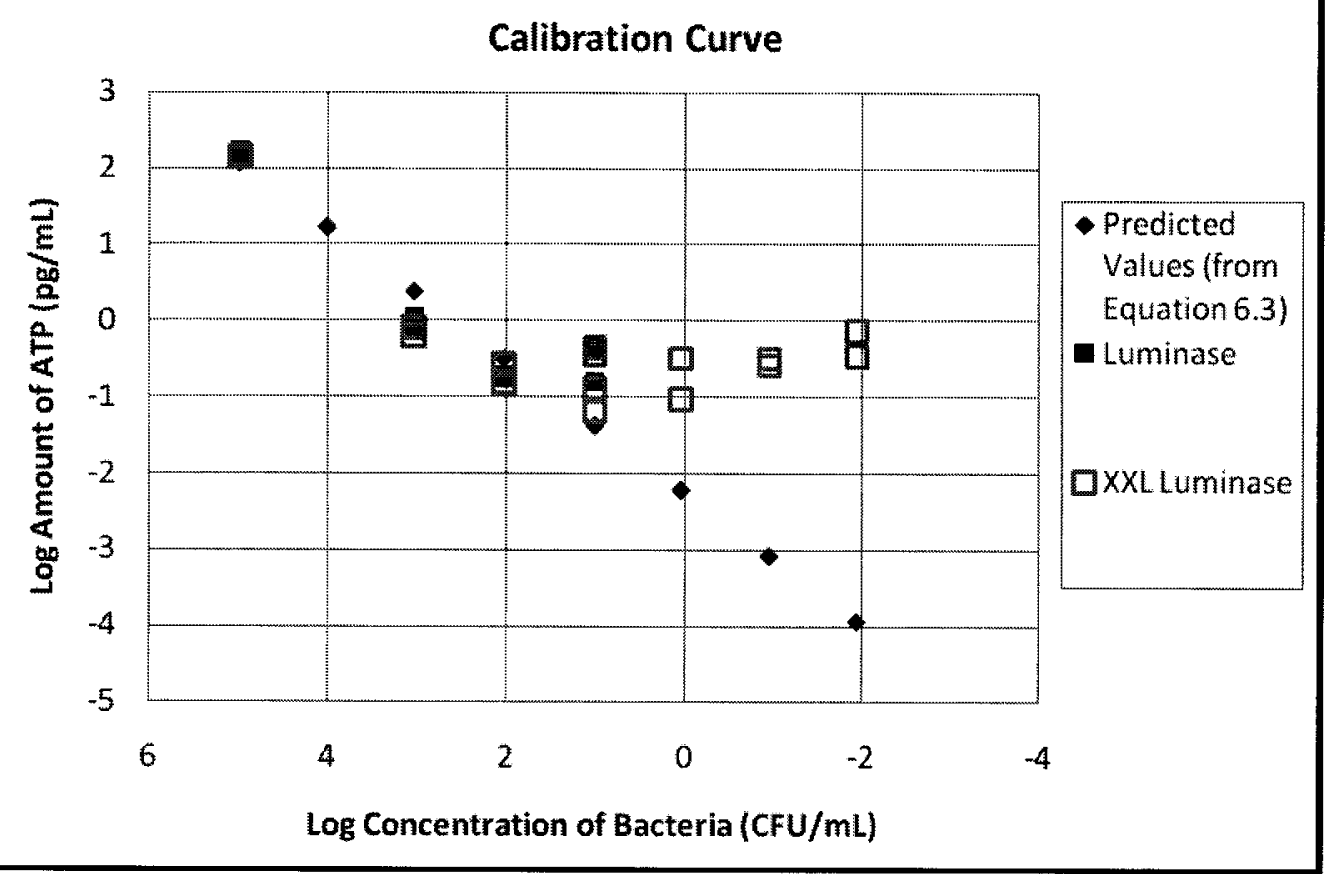

Figure 6.13: Calibration curve between membrane filtration and ATP assay using solutions with low bacteria concentrations $\left(10^{5}\right.$ to $\left.10^{-2} \mathrm{CFU} / \mathrm{mL}\right)$ and comparing regular strength Luminase with $\mathrm{XXL}$ Luminase.

To better define the sensitivity limit of the assay, a second calibration curve was created using only XXL Luminase. The bacteria concentrations of the solutions examined ranged from approximately $10^{3} \mathrm{CFU} / \mathrm{mL}$ to $10^{0} \mathrm{CFU} / 100 \mathrm{~mL}$.

The membrane filtration was performed for every solution and the average of three replicates is presented in Figure 6.14. The error bars represent the variance of the mean. The ATP assay was also performed in triplicate with XXL Luminase with an UltraCheque1 value of $366,981 \pm 26,840$ RLU and large sample volumes.

It is important to note that the reason three replicates were not tried before was due to logistical reasons. There was only a finite amount of glassware, space in the autoclave, time for allowing the PBS solutions to cool before use and time to complete the tests (the 
filtration of volumes of up to $1,000 \mathrm{~mL}$ manually in increments of $50 \mathrm{~mL}$ was time consuming. In addition, each replicate of the assay costs approximately $\$ 5$ and replicates were minimized as much as possible.

Parallel membrane filtration and ATP assays were performed on sample solutions ranging from in $10^{3} \mathrm{CFU} / \mathrm{mL}$ to $10^{-2} \mathrm{CFU} / \mathrm{mL}$ (or $10^{0} \mathrm{CFU} / 100 \mathrm{~mL}$ ) in addition to a control solution of PBS. The membrane filtration showed no colonies of E.coli for the PBS solution and the average amount of light measured from $800.0 \mathrm{~mL}$ was $1,440 \pm 536 \mathrm{RLU}$ which is equivalent to $0.05 \pm 0.02 \mathrm{pg} / \mathrm{mL}$ of ATP. Thus, approximately $2 \mathrm{RLU} / \mathrm{mL}$ measured can be attributed to PBS. The background levels of the reagents were measured at $138 \mathrm{RLU}$.

The calibration curve presented in Figure 6.15 shows that the sensitivity of the ATP assay using large sample volumes and XXL Luminase is likely to be fount between $1.8 \times 10^{2}$ and $1.8 \times 10^{1} \mathrm{CFU} / \mathrm{mL}$ (or between 2.25 and $1.25 \log$ of the bacteria concentration). This was a substantial increase from previous level of detection limit of $10^{5} \mathrm{CFU} / \mathrm{mL}$ and statistical analysis was used to further examine the detection limit.

To begin, a one-way ANOVA using the amount of ATP variable found that the sample means of the solutions tested were not from the same population $\left(p=2.4 \times 10^{-6}\right)$. A postANOVA Dunnett test was then performed to assess which of the data points was significantly different from the PBS control. It was found that only the solutions with bacteria concentration greater than $10^{2} \mathrm{CFU} / \mathrm{mL}$ were significantly different from PBS (see Table 6.3). From this, it is possible to conclude that the use of larger sample volumes 
and XXL Luminase has increased the detection limit of the ATP assay from $10^{5} \mathrm{CFU} / \mathrm{mL}$ to $10^{2} \mathrm{CFU} / \mathrm{mL}$.

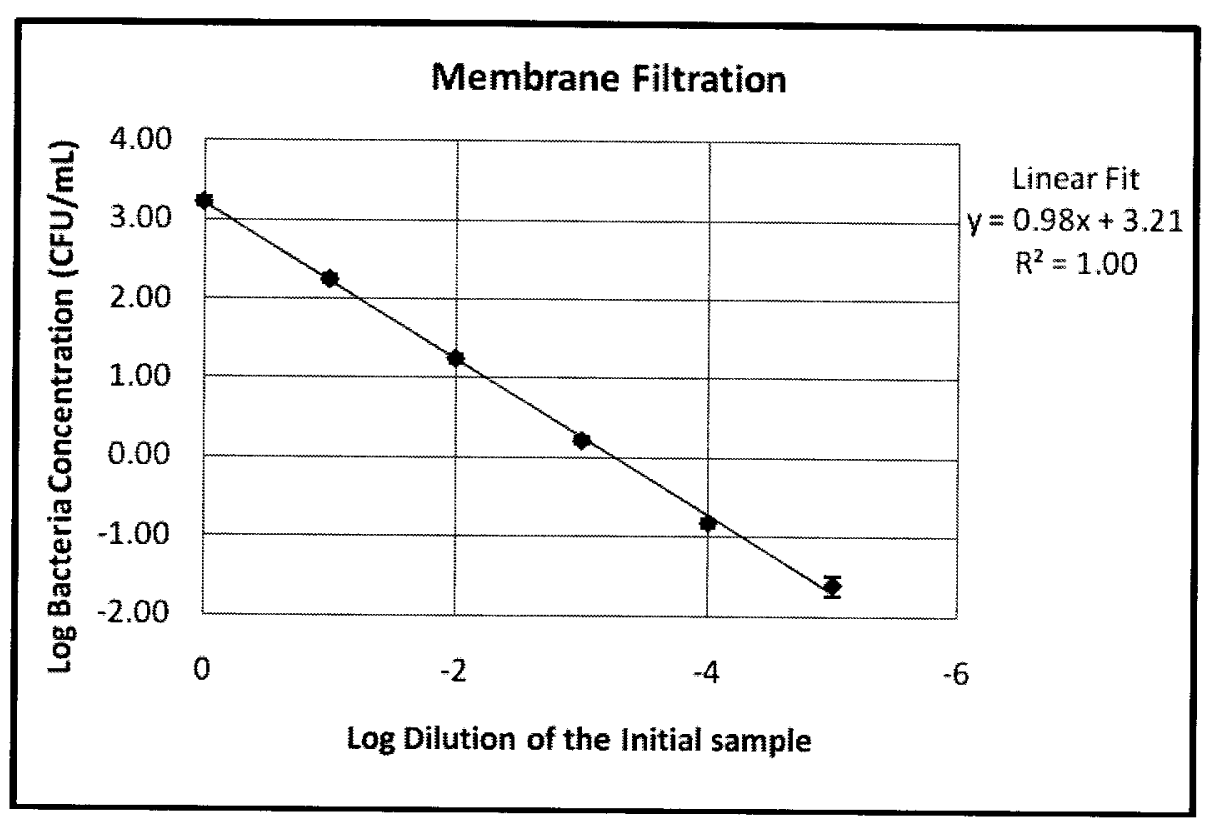

Figure 6.14: Results of the membrane filtration showing the logarithm bacteria concentration of the logarithm of six dilutions of a sample with initial bacteria concentration of $1.7 \times 10^{3} \pm 2 \times 10^{2} \mathrm{CFU} / \mathrm{mL}$ with PBS and low bacteria concentrations. 


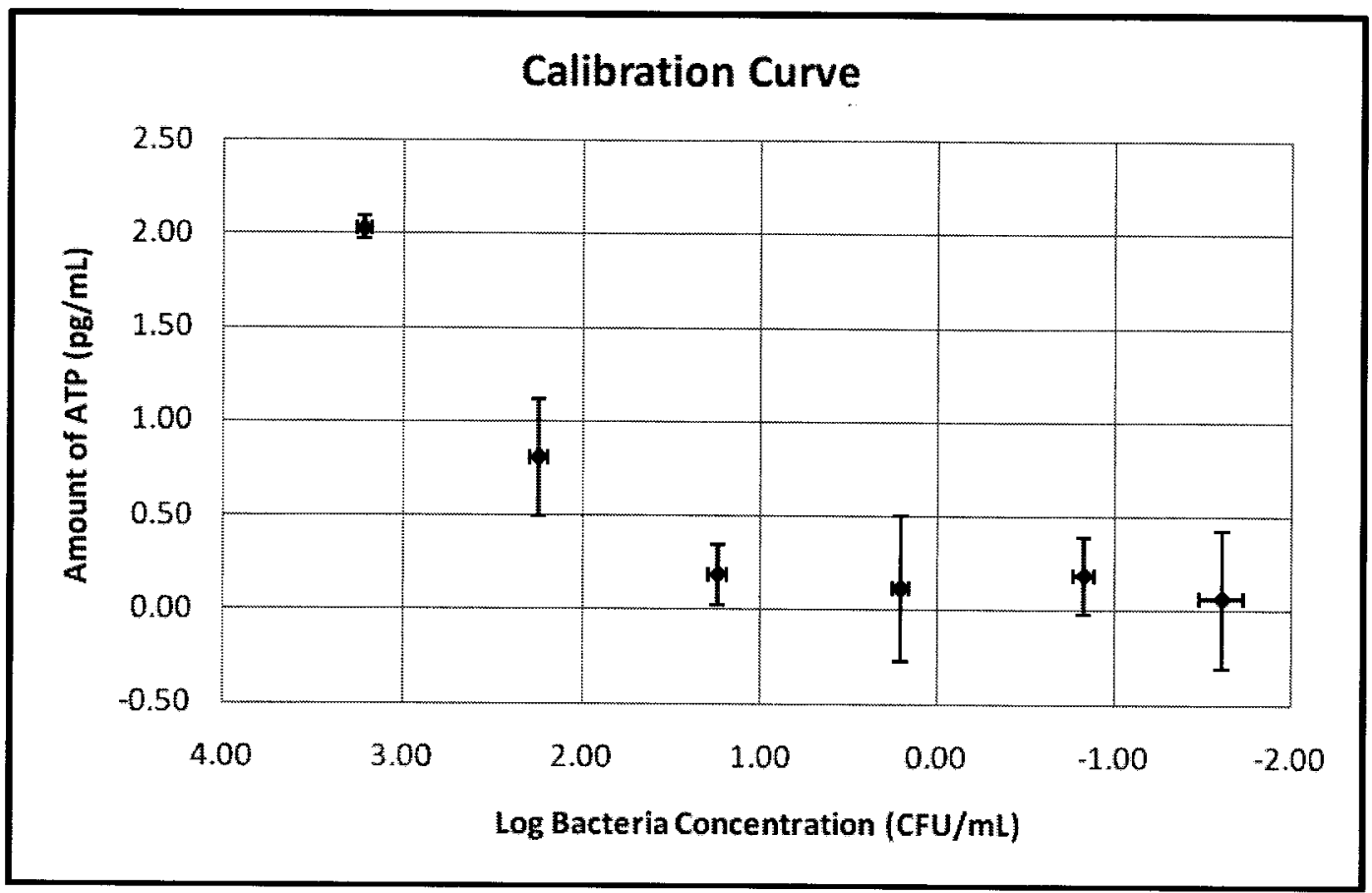

Figure 6.15: Calibration curve between membrane filtration and ATP assay using solutions with low bacteria concentrations $\left(1.7 \times 10^{3} \pm 2 \times 10^{2} \mathrm{CFU} / \mathrm{mL}\right)$ and $X X L$ Luminase.

Table 6.3: Results from the post-ANOVA Dunnett test comparing individual data points with the control solution, PBS (significance level is set at 0.05 ).

\begin{tabular}{|l|r|}
\hline Category & $p$-value \\
\hline PBS vs. $10^{3} \mathrm{CFU} / \mathrm{mL}$ & 0.000 \\
\hline PBS vs. $10^{2} \mathrm{CFU} / \mathrm{mL}$ & 0.046 \\
\hline PBS vs. $10^{1} \mathrm{CFU} / \mathrm{mL}$ & 0.986 \\
\hline PBS vs. $10^{0} \mathrm{CFU} / \mathrm{mL}$ & 1.000 \\
\hline PBS vs. $10^{-1} \mathrm{CFU} / \mathrm{mL}$ & 0.986 \\
\hline PBS vs. $10^{-2} \mathrm{CFU} / \mathrm{mL}$ & 1.000 \\
\hline
\end{tabular}




\subsubsection{CALIBRATION CURVE FOR HIGH BACTERIA CONCENTRATIONS WITH XXL LUMINASE}

This calibration curve examined solutions with high bacteria concentrations. The purpose of this curve was twofold: first, to assess the reproducibility of the results obtained in Section 6.1.6 and second, to assess the use of XXL Luminase for high bacteria concentrations.

Membrane filtration was performed for the $10^{5} \mathrm{CFU} / \mathrm{mL}$ bacteria concentrations, which was found to have $9.5 \times 10^{4} \pm 8 \times 10^{3} \mathrm{CFU} / \mathrm{mL}$. Other bacteria concentrations were estimated with the line of best of fit of the membrane filtration in Section 6.1 .5 (see Equation 6.2). From this equation, the concentration of the first data point was estimated to be $1.9 \times 10^{8} \mathrm{CFU} / \mathrm{mL}$ which was confirmed by the results of the membrane filtration to be $1.7 \times 10^{8} \pm 5 \times 10^{7} \mathrm{CFU} / \mathrm{mL}$ (a difference of $2 \times 10^{7} \mathrm{CFU} / \mathrm{mL}$ or approximately $11 \%$ ).

Two solutions were tested, $10^{5}$ and $10^{6} \mathrm{CFU} / \mathrm{mL}$, with the regular strength Luminase and sample volumes of $50.0 \mathrm{~mL}$ and $1.0 \mathrm{~mL}$ respectfully. The results of this trial were estimated using the equation of the calibration curve for high bacteria concentrations determined in Section 6.1.6 (see Equation 6.3). The actual and predicted amounts of ATP, as seen in Table 6.4, show that the actual amounts of ATP are slightly higher than the predicted values. However they have similar orders of magnitude and are considered acceptable.

Four solutions were tested with XXL Luminase: $10^{5}, 10^{6}, 10^{7}$ and $10^{8} \mathrm{CFU} / \mathrm{mL}$. The sample volume for the $10^{5}$ solutions was $50.0 \mathrm{~mL}$ however the sample volumes were significantly reduced for subsequent solutions due to the limits of the luminometer being reached. For the $10^{6} \mathrm{CFU} / \mathrm{mL}$ samples, the volume was reduced to $1.0 \mathrm{ml}$ and sample 
volume of the $10^{7}$ and $10^{8}$ solutions was reduced to $0.10 \mathrm{~mL}$. The resulting calibration curve, see Figure 6.16, shows that all but one solution measured with XXL Luminase had a good correlation with both the previous and current high bacteria concentrations measured with regular strength Luminase. The outlier data point may because the small sample volumes required to remain within the limits of the luminometer. Thus, for bacteria concentrations larger than $10^{6}$ it is best to use regular strength Luminase.

Table 6.4: Predicted and actual amounts of ATP using regular strength Luminase and XXL Luminase

\begin{tabular}{|r|r|r|r|}
\hline $\begin{array}{l}\text { Bacteria } \\
\text { Concentration } \\
(\mathrm{CFU} / \mathrm{mL})\end{array}$ & $\begin{array}{l}\text { Predicted Amount of ATP } \\
(\mathrm{pg} / \mathrm{mL})\end{array}$ & $\begin{array}{l}\text { Average Amount of } \\
\text { ATP using Luminase } \\
(\mathrm{pg} / \mathrm{mL})\end{array}$ & $\begin{array}{l}\text { Average Amount of } \\
\text { ATP using XXL } \\
\text { Luminase } \\
(\mathrm{pg} / \mathrm{mL})\end{array}$ \\
\hline $9.5 \times 10^{4}$ & 117 & 169 & 142 \\
\hline $1.4 \times 10^{6}$ & 1,232 & 2,170 & 2,128 \\
\hline $1.6 \times 10^{7}$ & 10,297 & & 32,677 \\
\hline $1.9 \times 10^{8}$ & 86,080 & & 23,797 \\
\hline
\end{tabular}




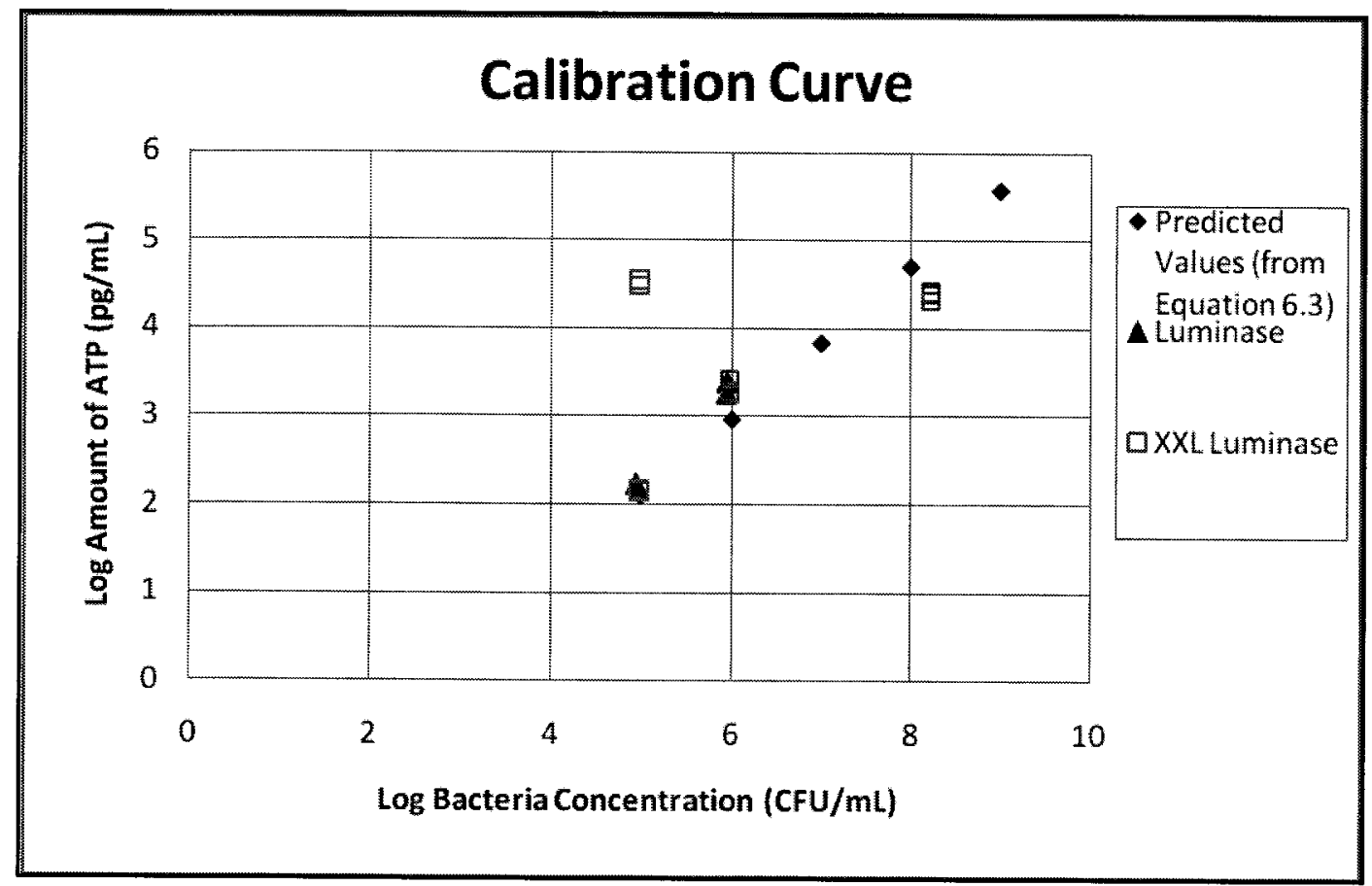

Figure 6.16: Calibration curve between membrane filtration and ATP assay using solutions with high bacteria concentrations $\left(10^{5}\right.$ to $\left.10^{9} \mathrm{CFU} / \mathrm{mL}\right)$ and comparing regular strength Luminase with XXL Luminase.

\subsection{Phase II: UV InACTIVATION EXPERIMENTS}

The purpose of Phase II of the project was to investigate the potential of the ATP assay as a tool for monitoring UV disinfection of water and wastewater. During the field trials, LuminUltra investigated the use of pretreatment methods, which were applied after UV irradiation and before the ATP assay (see Table A. 1) to optimize the use of the ATP assay for UV disinfection monitoring. Using the initial field trials as a starting point, the experimental design of Phase II consisited of four parts that aimed to: (1) evaluate the use of the ATP assay immediately after UV treatment, (2) determine the the effect of the incubation temperature applied after UV treatment, (3) determine the effect of incubation time allowed after UV treatment, and (4) evaluate other pre-tretment methods. 


\subsubsection{UV DOSE RESPONSE CURVES}

Presented here are the results of two series of UV dose-response curves. The UV doseresponse curves were created by exposing a sample to a wide range of UV doses ( 0 to 100

$\mathrm{mJ} / \mathrm{cm}^{2}$ ) and measuring the bacteria count by membrane filtration (see Section 5.4) and the ATP quantity by the ATP assay (see Section 5.5). Unless otherwise noted, each UV irradiation experiment was repeated three times and the artithmetic mean presented in the figures with the variance represented by the error bars.

The first series of irradiation experiments measured the inactivation effect of increasing UV doses on a sample with an initial E.coli concentration of $1.3 \times 10^{5} \pm 3 \times 10^{4} \mathrm{CFU} / \mathrm{mL}$ (as confirmed by membrane filtration). The inactivation curve obtained is presented in Figure 6.17 where the logarithm of the number of surviving E.coli bacteria (y-axis), measured in colony forming units per millilitre, is plotted against corresponding UV dose (x-axis), measured in millijoules per centimetre squared. As expected, increasing the UV dose increases the inactivation rate of bacteria. The inactivation of bacteria is rapid for the first $20 \mathrm{~mJ} / \mathrm{cm}^{2}$ of applied UV dose, after which the inactivation become more gradual. The largest amounts of inactivation are seen at 80 and $100 \mathrm{~mJ} / \mathrm{cm}^{2}$. In water and wastewater treatment plants, typically a UV dose of $40 \mathrm{~mJ} / \mathrm{cm}^{2}$ and greater is used for inactivation.

The curve created by measuring the cellular ATP of samples after UV exposure is presented in Figure 6.18. The amount of ATP measured (y-axis), measured in picograms $\left(1 \mathrm{pg}=10^{-12} \mathrm{~g}\right)$ per millilitre, is plotted against the corresponding UV dose ( $\mathrm{x}$-axis) measured in millijoules per centimetre squared $\left(\mathrm{mJ} / \mathrm{cm}^{2}\right)$. If $U V$ irradiation causes similar damage to cellular ATP as it does to the DNA of the cells, then the trend observed in 
Figure 6.18 would be expected to be seen here in Figure 6.18. However, this was not the case. In the latter figure, the initial quantity of ATP was approximately $200 \mathrm{pg} / \mathrm{mL}$. Following, the ATP value fluctuated between 150 and $524 \mathrm{pg} / \mathrm{mL}$. It should however be noted that the sample volume used for the ATP test was $5 \mathrm{~mL}$ which was at the lower end of the suggested range $(5-100 \mathrm{~mL})$. Increasing the sample volume may increase the detection of the changes that may be occurring in cellular ATP.

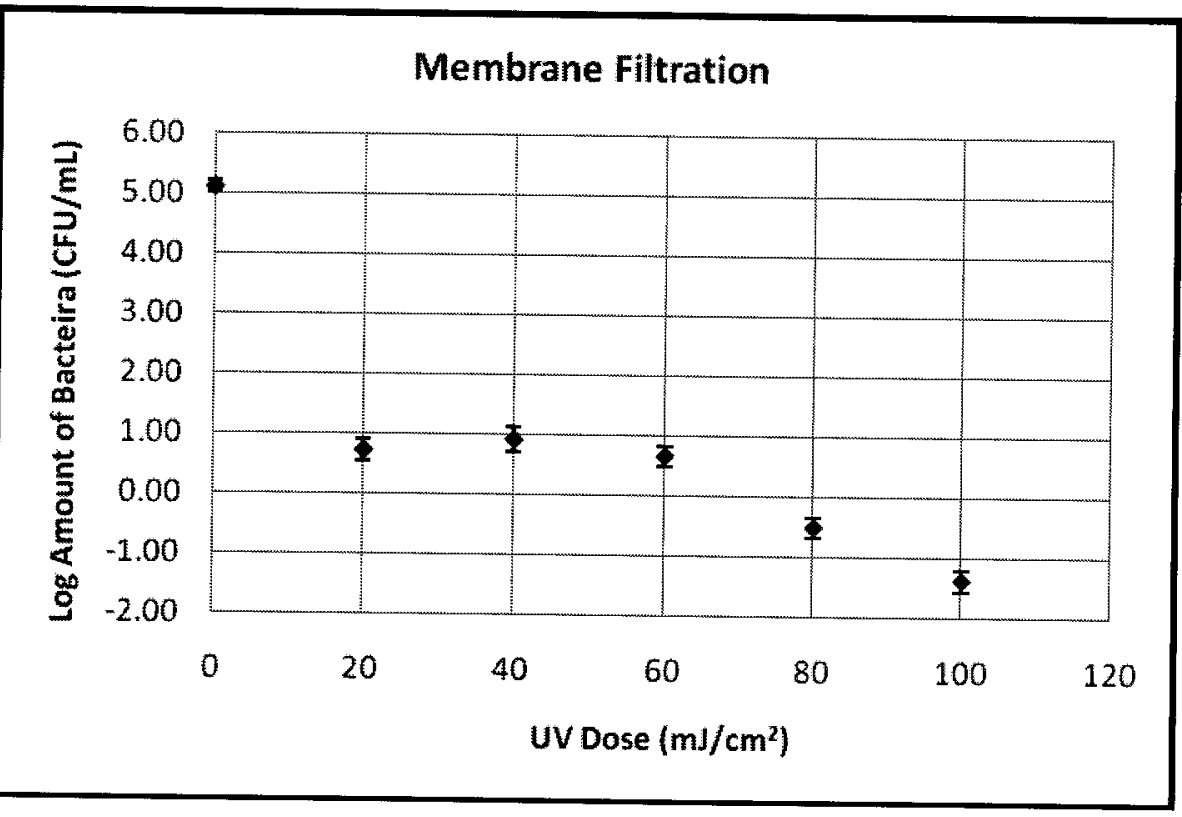

Figure 6.17: UV dose-response of an initial solution of $1.3 \times 10^{5} \pm 3 \times 10^{4} \mathrm{CFU} / \mathrm{mL}$ showing the $\log$ of the amount of bacteria as measured by membrane filtration and $0.1 \%$ peptone water 


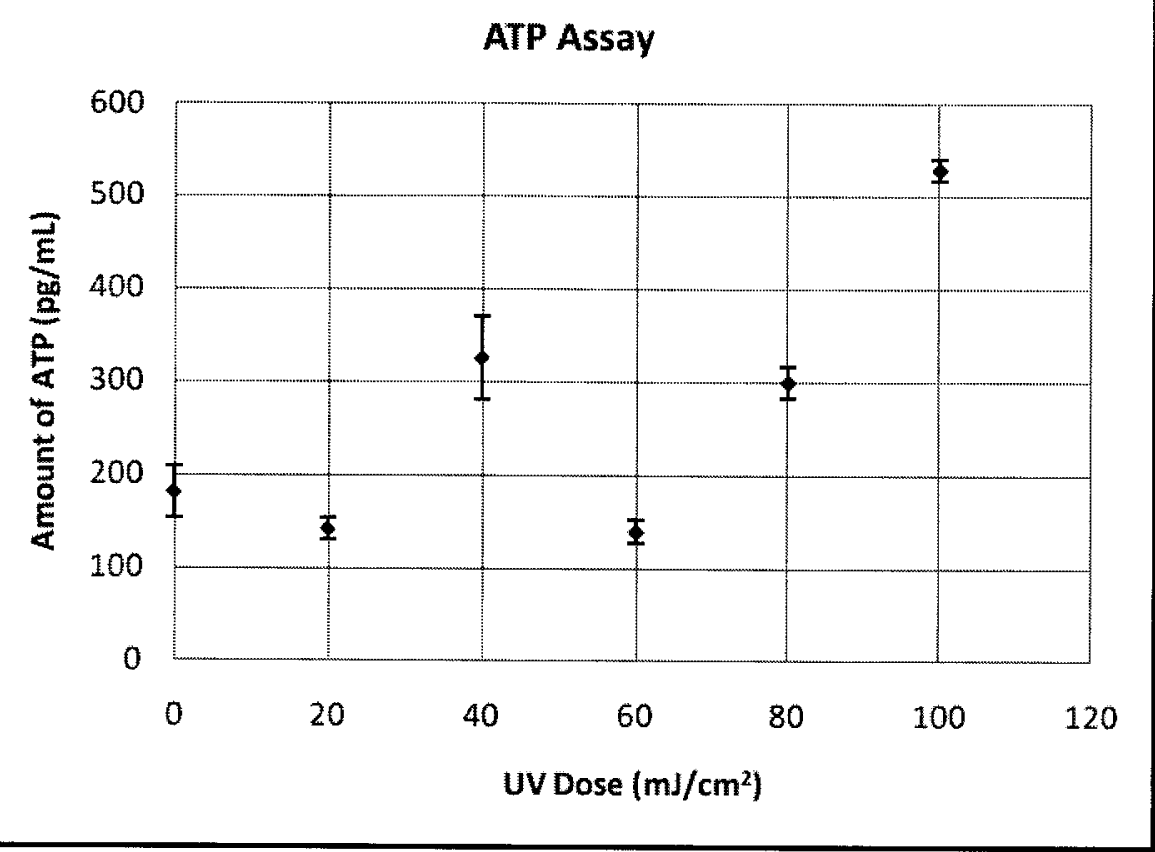

Figure 6.18: $\mathrm{UV}$ dose-response of an initial solution of $1.3 \times 10^{5} \pm 3 \times 10^{4} \mathrm{CFU} / \mathrm{mL}$ showing the amount of ATP as measured by ATP assay with $5.0 \mathrm{~mL}$ samples and $0.1 \%$ peptone water and Luminase.

The previous experiments, presented in Figure 6.17 and Figure 6.18, were repeated by using XXL Luminase and larger sample volumes of $10.0 \mathrm{~mL}$. Due to the experimental setup of the UV reactor (see Section 5.7.3) the sample volume to irradiate was limited and a maximum of $10 \mathrm{~mL}$ was able to be allocated to the ATP assay. The bacteria concentration of the initial sample solution was measured to be $1.5 \times 10^{5} \pm 10^{4} \mathrm{CFU} / \mathrm{mL}$, confirmed by membrane filtration.

The UV dose-response curve formulated from the results of the membrane filtration is shown in Figure 6.19. As expected, there was a decrease in culturable bacteria with an increase in UV exposure and even at a UV dose of $20 \mathrm{~mJ} / \mathrm{cm}^{2}$ there is a significant decrease in the inactivation of bacteria. The inactivation curve was very similar to the previously observed inactivation curve (Figure 6.17) which confirms the reproducibility 
of the data. However, a similar trend was not observed when the measured ATP quantities were plotted against the UV dose (Figure 6.20). If UV irradiation has no effect on the ATP of cells, then the UV dose-response curve would resemble a horizontal line and there would be no significant difference between the amounts of ATP measured before and after UV irradiation. An ANOVA test was performed on the data of the ATP assay and it concluded that there was a significant difference between the means of the samples measured at different UV doses $(p=0.039)$. However, comparing each sample with that of the Dose 0 sample, using Dunnett's test, no sample was found to be significantly different than the Dose 0 sample, see Table 6.4. The difference that was measured in the ANOVA test was found to be between Dose 20 and Dose 60, as confirmed by a Tukey test $(p=0.047)$. Overall, the statistical analysis suggest that UV irradiation does not have a significant effect on the ATP content or activity of the microbial cells.

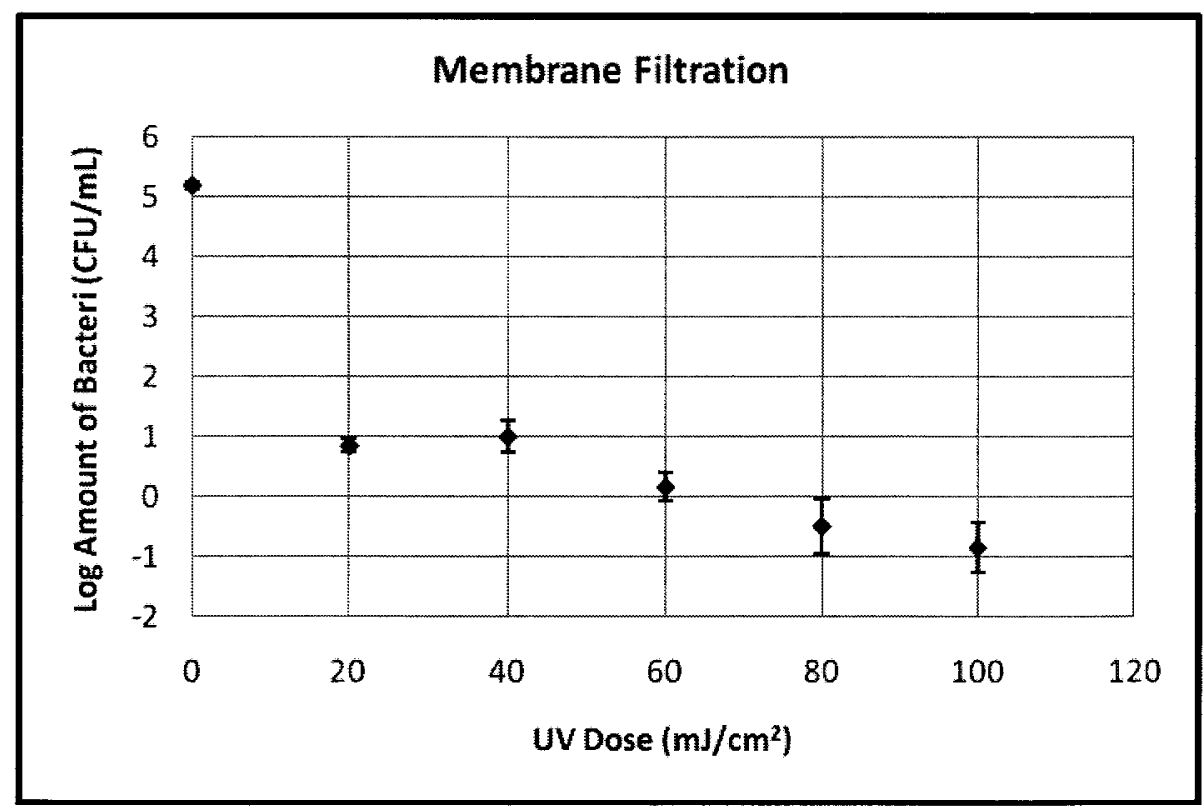

Figure 6.19: UV dose-response of an initial solution of $1.5 \times 10^{5} \pm 2 \times 10^{4} \mathrm{CFU} / \mathrm{mL}$ showing the log of the amount of bacteria as measured by membrane filtration and PBS 


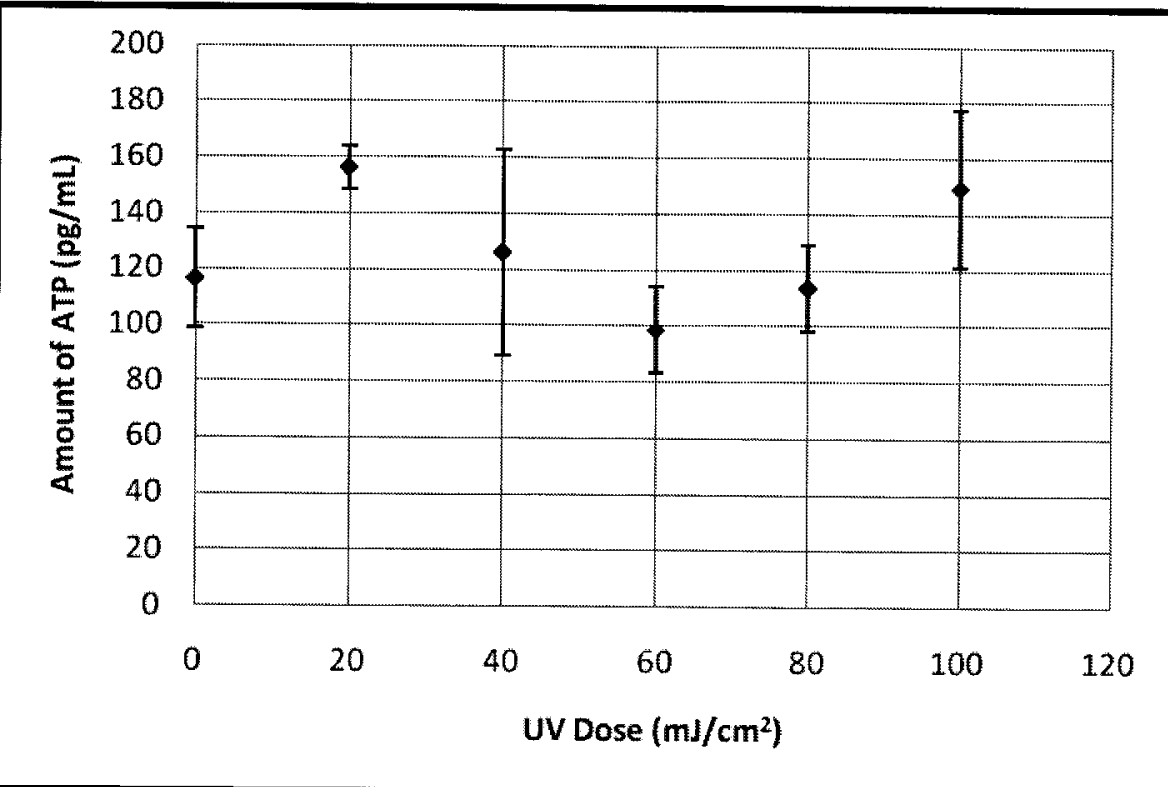

Figure 6.20: UV dose-response of an initial solution of $1.3 \times 10^{5} \pm 3 \times 10^{4} \mathrm{CFU} / \mathrm{mL}$ showing the amount of ATP as measured by the ATP assay with PBS and XXL Luminase

Table 6.5: Results of the Dunnett's test using the ATP assay comparing sample points to that of the original sample

\begin{tabular}{|l|r|}
\hline Category & $\boldsymbol{p}$ - value \\
\hline Dose 0 vs. Dose 20 & 0.129 \\
\hline Dose 0 vs. Dose 40 & 0.978 \\
\hline Dose 0 vs. Dose 60 & 0.743 \\
\hline Dose 0 vs. Dose 80 & 1.000 \\
\hline Dose 0 vs. Dose 100 & 0.247 \\
\hline
\end{tabular}




\subsubsection{EFFECT OF INCUBATION TIME}

The purpose of this experiment was to determine the effect of incubation time on the amount of ATP measured from a UV disinfected sample. It was suspected that changes initiated by UV irradiation on cellular ATP may require some time for completion, and an incubation period may be required to observe a significant decrease in ATP activity to occur. Subsequent to an incubation period, the ATP activity may decrease, which would improve the potential use of the ATP assay as a monitoring tool.

Three incubation temperatures were considered: $4^{\circ} \mathrm{C}, 21^{\circ} \mathrm{C}$ and $35^{\circ} \mathrm{C}$. In order to select the incubation temperature, a short experiment was performed. An initial sample was prepared that contained approximately $2.2 \times 10^{5} \mathrm{CFU} / \mathrm{mL}$ E.coli bacteria, confirmed by membrane filtration (see procedures in Section 5.4). Three samples were exposed to UV irradiation of a dose of $100 \mathrm{~mJ} / \mathrm{cm}^{2}$ and incubated at the aforementioned temperatures for six hours. The increase of the bacteria concentration during that time period was measured by membrane filtration and the average of two replicates of each sample are shown in Figure 6.21.

As suspected, the sample incubated at $35^{\circ} \mathrm{C}$, which is near the optimal growth temperature of E.coli of $37^{\circ} \mathrm{C}$, showed the highest growth and the sample incubated at $4^{\circ} \mathrm{C}$ showed the lowest growth. To have the best opportunity to see the effect of incubation, initial incubation trials were carried out at $4^{\circ} \mathrm{C}$. 


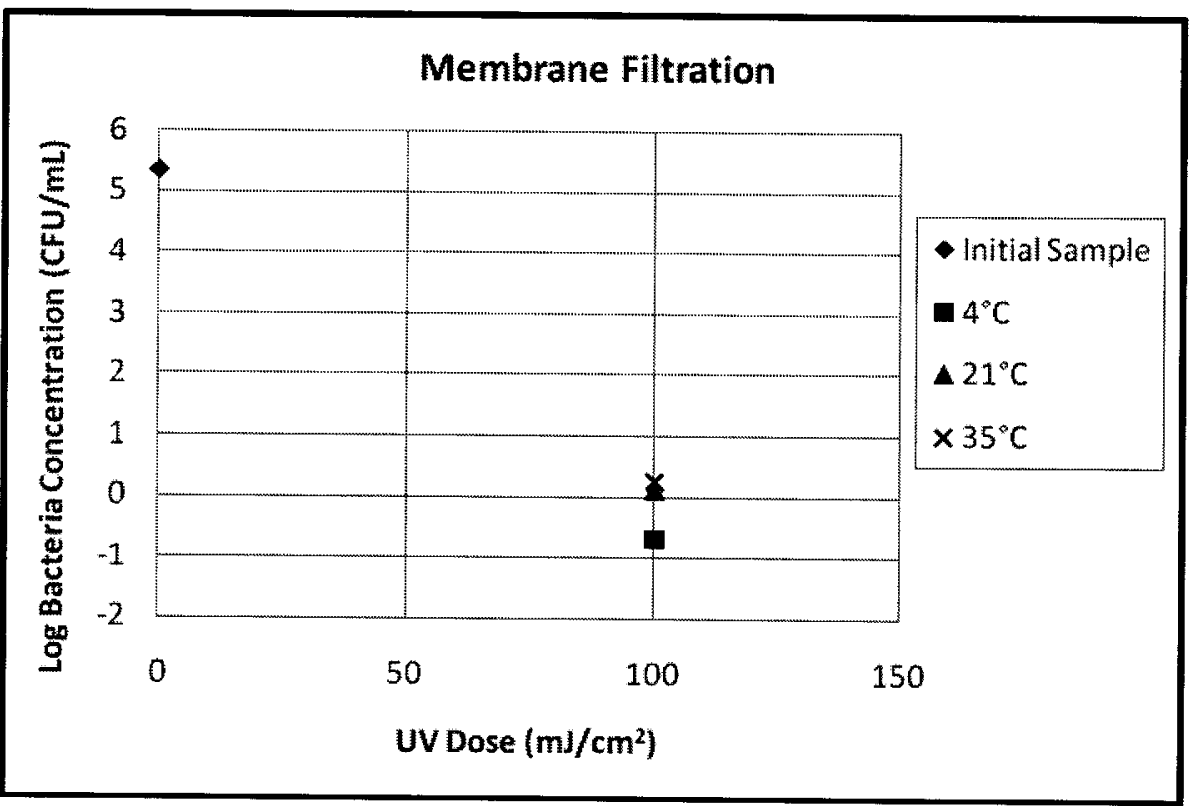

Figure 6.21: Results of 6-hour incubation at three different temperatures $\left(4^{\circ} \mathrm{C}, 21^{\circ} \mathrm{C}\right.$ and $\left.35^{\circ} \mathrm{C}\right)$ of an initial sample with a bacteria concentration of $2.2 \times 10^{5} \mathrm{CFU} / \mathrm{mL}$ exposed to a $\mathrm{UV}$ dose of $100 \mathrm{~mJ} / \mathrm{cm}^{2}$.

The incubation-time trials were performed with three UV doses: 0,40 and $60 \mathrm{~mJ} / \mathrm{cm}^{2}$. The dose of $40 \mathrm{~mJ} / \mathrm{cm}^{2}$ was selected because it is most often used in water disinfection, and the dose of $60 \mathrm{~mJ} / \mathrm{cm}^{2}$ was selected because it is frequently used for wastewater disinfection, while the dose of $0 \mathrm{~mJ} / \mathrm{cm}^{2}$ was selected as the control. An initial solution was prepared that had a bacteria concentration of $1.69 \times 10^{6} \pm 2.0 \times 10^{5} \mathrm{CFU} / \mathrm{mL}$. Sample volumes of $20.0 \mathrm{~mL}$ were then irradiated with the selected UV doses following the procedure described in Section 5.7.3. After irradiation, samples were covered with aluminum foil to prevent light-induced repair and regrowth, and incubated at $4{ }^{\circ} \mathrm{C}$ for periods between approximately 4 hours and 144 hours (or 6 days). After each incubation period, $5.0 \mathrm{~mL}$ of each sample was measured with the ATP assay using Luminase and the remaining volume was used for membrane filtration; see Section 5.5 and Section 5.4 for procedural details of the ATP assay and membrane filtration respectfully. 
Results of the membrane filtration for doses of 0,40 and $60 \mathrm{~mJ} / \mathrm{cm}^{2}$ are shown in Figure 6.22, Figure 6.23 and Figure 6.24 respectfully, where the log of the bacteria concentration (y-axis) is plotted against the incubation time (x-axis). As expected, the bacteria concentration of samples not exposed to UV light (see Figure 6.22) remain unchanged during all incubation periods. The samples that were irradiated at 40 and $60 \mathrm{~mJ} / \mathrm{cm}^{2}$ (see Figure 6.23 and Figure 6.24) showed some variation during the incubation time but no significant changes in bacteria counts were observed.

Corresponding results for the ATP assay are seen in Figure 6.25, Figure 6.26 and Figure 6.27 for UV doses of 0,40 and $60 \mathrm{~mJ} / \mathrm{cm}^{2}$ respectfully where the amount of ATP (x-axis) is plotted against the incubation time (y-axis). The figure for the samples irradiated at 40 $\mathrm{mJ} / \mathrm{cm}^{2}$ (see Figure 6.26) showed that the initial amount of ATP began at about 1,000 $\mathrm{pg} / \mathrm{mL}$, peaked at $5,500 \mathrm{pg} / \mathrm{mL}$ (at 6 hours) and gradually decreased. To a lesser extent, the same trend can be seen in Figure 6.27, which shows the results for dose $60 \mathrm{~mJ} / \mathrm{cm}^{2}$. The amount of ATP began at $1,700 \mathrm{pg} / \mathrm{mL}$, peaked at $3,800 \mathrm{pg} / \mathrm{mL}$ (at 9 hours) and gradually decreased. The sample that was not exposed to UV irradiation had similar ATP concentrations compared to the samples irradiated at 40 and $60 \mathrm{~mJ} / \mathrm{cm}^{2}$, which indicates that the UV exposure did not cause an immediate decrease in cellular ATP. The initial increase in the amount of ATP may be due to the enzyme repair mechanisms of cells observed after UV irradiation known as light and dark repair. As the cell gradually repaired itself, the activity of the cell increased as does the production of ATP. The observed decrease in the amount of ATP is thought to be caused by the expected decrease in cellular activity after UV irradiation. It appears that there were competing cellular mechanisms occurring simultaneously. 
To be able to use the ATP assay as a monitoring tool for UV disinfection, there should be a significant difference between the ATP measurements before and after UV irradiation. The observed increase and subsequent decrease in the amounts of ATP observed after irradiation was not significantly different than the amounts of ATP of samples not exposed to irradiation and the incubation time did not seem to improve the use of the ATP assay as a monitoring tool after UV irradiation.

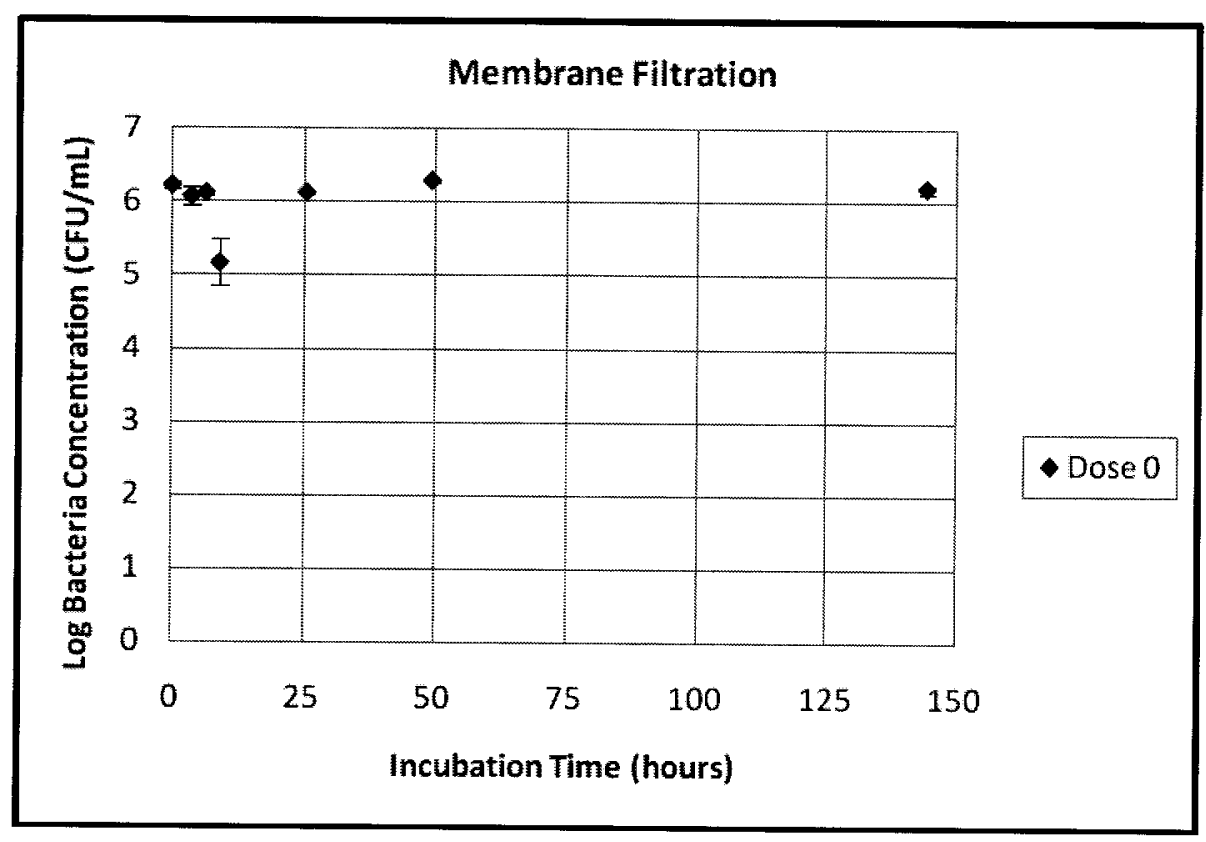

Figure 6.22: Results of the membrane filtration of a sample with initial bacterial concentration of $1.69 \times 10^{6} \pm 2.0 \times 10^{5} \mathrm{CFU} / \mathrm{mL}$ exposed to no $\mathrm{UV}$ irradiation and incubated at $4^{\circ} \mathrm{C}$ for up to 144 hours $(6$ days). 


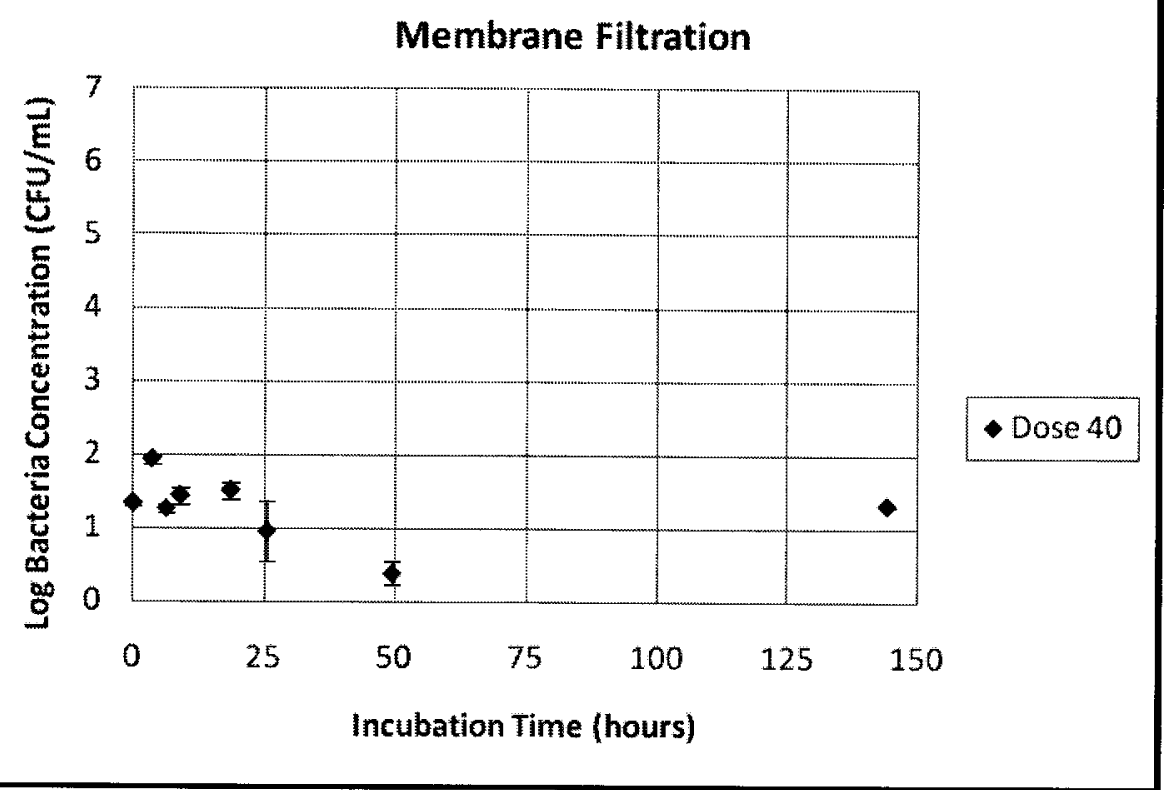

Figure 6.23: Results of the membrane filtration of a sample with initial bacterial concentration of $1.69 \times 10^{6} \pm 2.0 \times 10^{5} \mathrm{CFU} / \mathrm{mL}$ exposed to $\mathrm{UV}$ radiation of a dose of $40 \mathrm{~mJ} / \mathrm{cm}^{2}$ and incubated at $4^{\circ} \mathrm{C}$ for up to 144 hours (or 6 days).

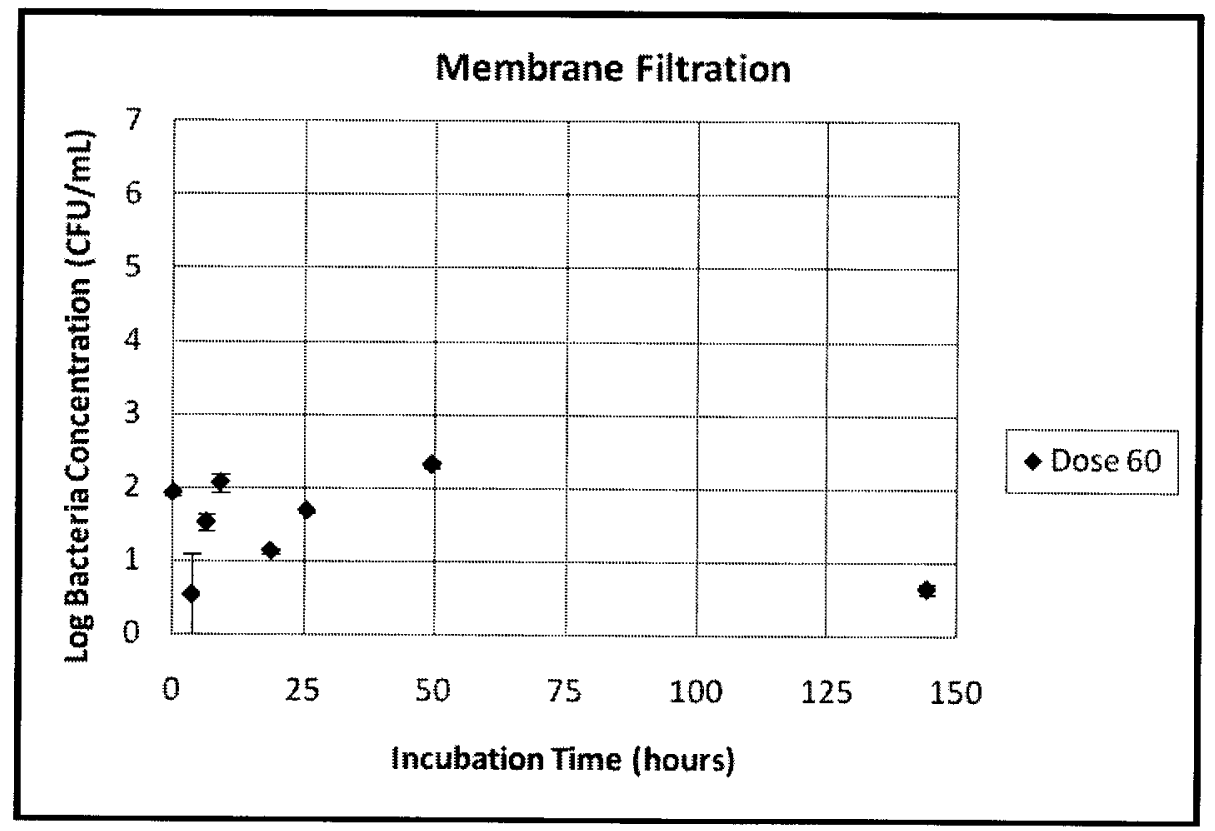

Figure 6.24: Results of the membrane filtration of a sample with initial bacterial concentration of $1.69 \times 10^{6} \pm 2.0 \times 10^{5} \mathrm{CFU} / \mathrm{mL}$ exposed to $\mathrm{UV}$ radiation of a dose of $60 \mathrm{~mJ} / \mathrm{cm}^{2}$ and incubated at $4^{\circ} \mathrm{C}$ for up to 144 hours (or 6 days). 


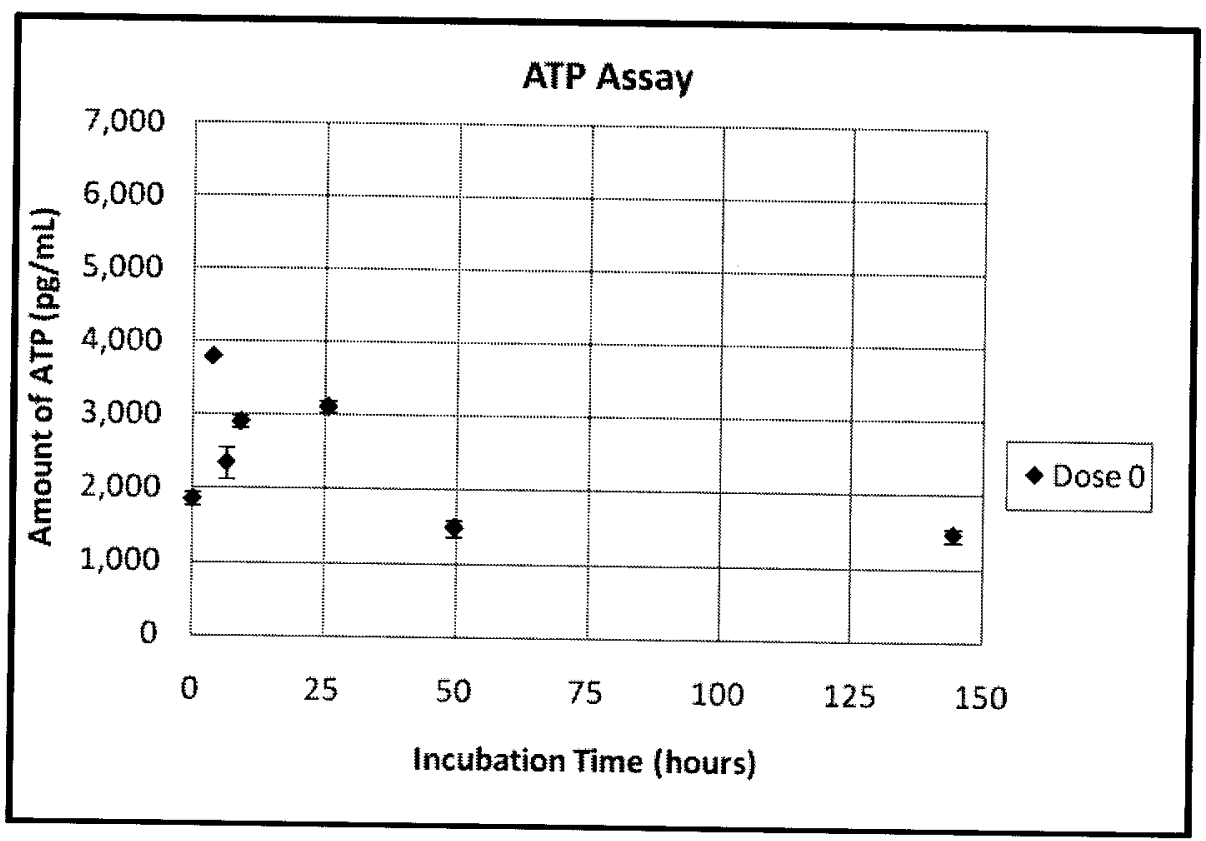

Figure 6.25: Results of the ATP assay of a sample with initial bacterial concentration of $1.69 \times 10^{6} \pm$ $2.0 \times 10^{5} \mathrm{CFU} / \mathrm{mL}$, as measured by membrane filtration, exposed to UV radiation of a dose of $0 \mathrm{~mJ} / \mathrm{cm}^{2}$ and incubated at $4^{\circ} \mathrm{C}$ for up to 144 hours (6 days).

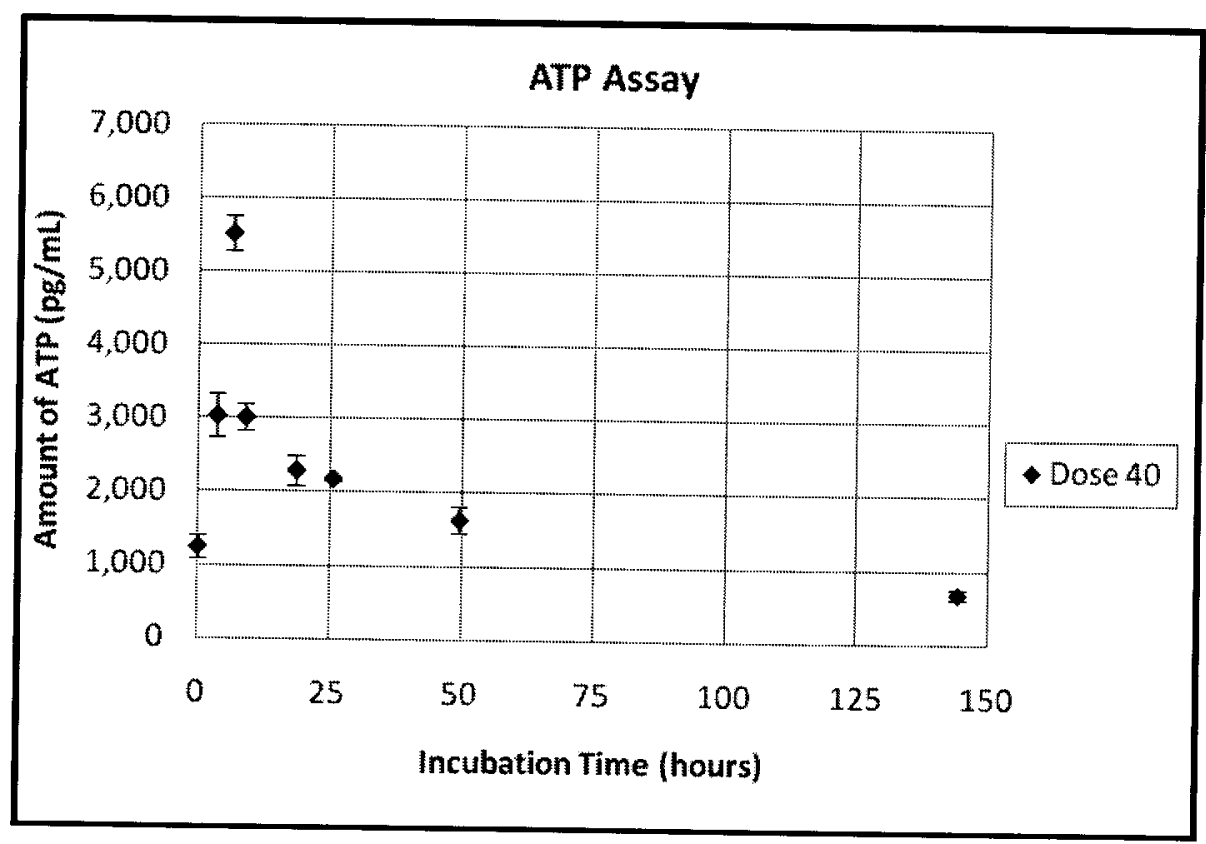

Figure 6.26: Results of the ATP assay of a sample with initial bacterial concentration of $1.69 \times 106 \pm$ $2.0 \times 105 \mathrm{CFU} / \mathrm{mL}$, as measured by membrane filtration, exposed to $\mathrm{UV}$ radiation of a dose of 40 $\mathrm{mJ} / \mathrm{cm} 2$ and incubated at $4^{\circ} \mathrm{C}$ for up to 144 hours (6 days). 


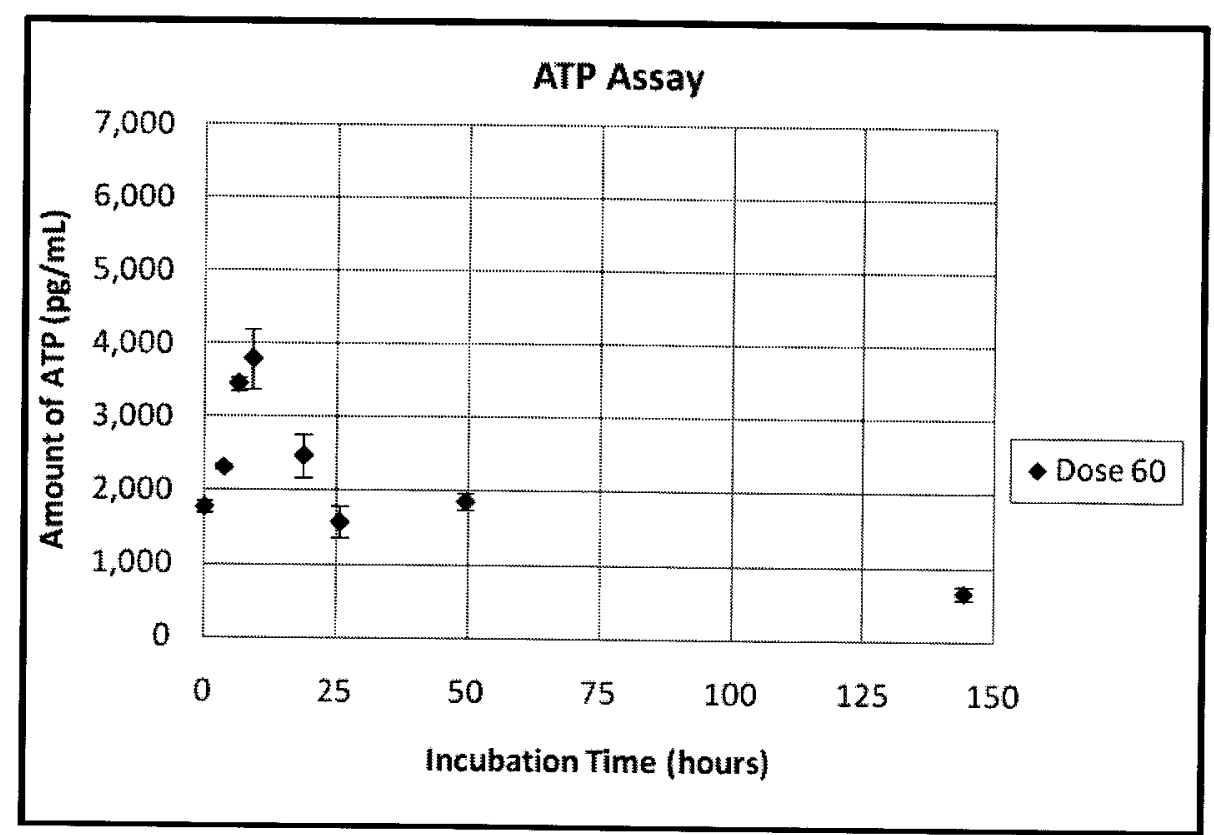

Figure 6.27: Results of the ATP assay of a sample with initial bacterial concentration of $1.69 \times 10^{6} \pm$ $2.0 \times 10^{5} \mathrm{CFU} / \mathrm{mL}$, as measured by membrane filtration, exposed to $\mathrm{UV}$ radiation of a dose of 60 $\mathrm{mJ} / \mathrm{cm}^{2}$ and incubated at $4^{\circ} \mathrm{C}$ for up to 144 hours ( 6 days).

\subsubsection{EFFECT OF INCUBATION AT ROOM TEMPERATURE}

The purpose of this experiment was to determine the effect of incubation at room temperature on the amount of ATP measured from a sample after UV irradiation. In addition, this experiment aimed to determine whether similar changes in ATP content would occur at $21^{\circ} \mathrm{C}$ as opposed to $4^{\circ} \mathrm{C}$. The incubation temperature of $35^{\circ} \mathrm{C}$ was not considered as a possible incubation temperature as this temperature would result in substantial regrowth.

The procedure for this experiment was similar to that of the previous incubation-time trials. An initial solution was prepared that had a bacteria concentration of $1.9 \times 10^{5} \pm$ $3 \times 10^{4} \mathrm{CFU} / \mathrm{mL}$. Sample volumes of $20.0 \mathrm{~mL}$ were then irradiated with UV dose of 40 following the procedure described in Section 5.7.3. The dose of $40 \mathrm{~mJ} / \mathrm{cm}^{2}$ was selected because during the incubation-time trials, the results showed the largest effect. After 
irradiation, the samples were covered with aluminum foil and incubated at ambient temperature for up to 26 hours. After each incubation period, $5.0 \mathrm{~mL}$ of each sample was measured with the ATP assay using Luminase and the required volume was used for membrane filtration (see Section 5.5 and Section 5.4 for procedural details of the ATP assay and membrane filtration respectfully).

It was expected that increasing the incubation temperature from $4{ }^{\circ} \mathrm{C}$ to $21^{\circ} \mathrm{C}$ would increase the level of cell activity. The experiment was ended with a sample incubated for 8 hours because after this time, the results of the membrane filtration showed regrowth of bacteria and any increase of ATP would be caused by the increase in bacteria levels.

Results of the membrane filtration in Figure 6.28 show the $\log$ of the bacteria concentration ( $\mathrm{x}$-axis) plotted against the incubation time (y-axis). As expected, there was a significant difference in the concentration of bacteria before and after UV irradiation, and there was little change in bacteria concentrations during the 8 hour incubation time. Up to 8 hours, incubating samples at $21^{\circ} \mathrm{C}$ did not cause significant regrowth in samples.

Results of the ATP assay can be seen in Figure 6.29 where the amount of ATP (x-axis) is plotted against the incubation time (y-axis). Following the observations of the incubationtime trials at $4^{\circ} \mathrm{C}$, an increase in the amount of ATP followed by a decrease in the amount of ATP was expected from irradiated samples. In addition, due to the increase in incubation temperature, this effect was expected sooner than at the previously observed 6 hour. The results showed a slight increase in the amount of ATP after irradiation which remained unchanged until approximately 4 hours after irradiation. It is also interesting to note that the observed increase in the amount of ATP after irradiation is approximately 
$700 \mathrm{pg} / \mathrm{mL}$ above the initial amount of ATP measured, which would not allow the ATP assay as an effective monitoring tool.

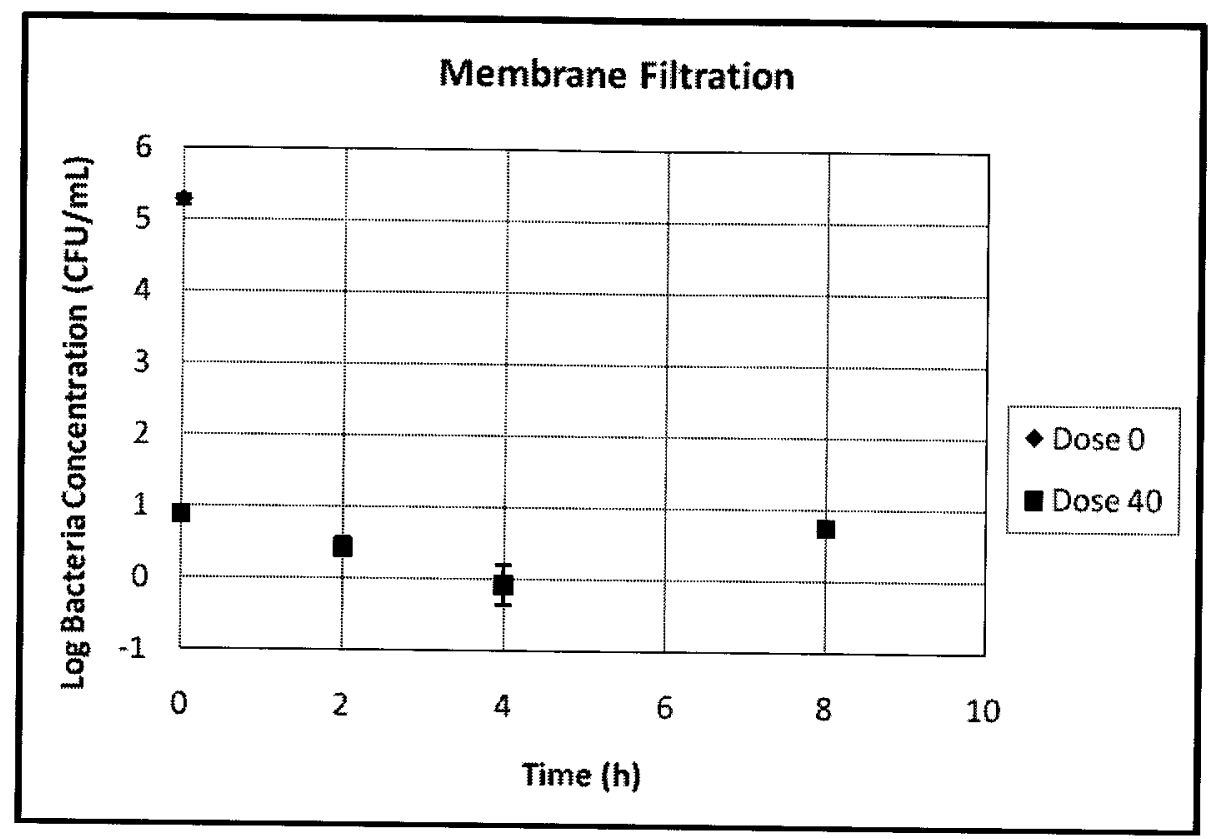

Figure 6.28: Results of the membrane filtration of a sample with initial bacterial concentration of 1 . $9 \times 10^{5} \pm 3 \times 10^{4} \mathrm{CFU} / \mathrm{mL}$ exposed to $\mathrm{UV}$ radiation of a dose of $40 \mathrm{~mJ} / \mathrm{cm}^{2}$ and incubated at room temperature for 8 hours.

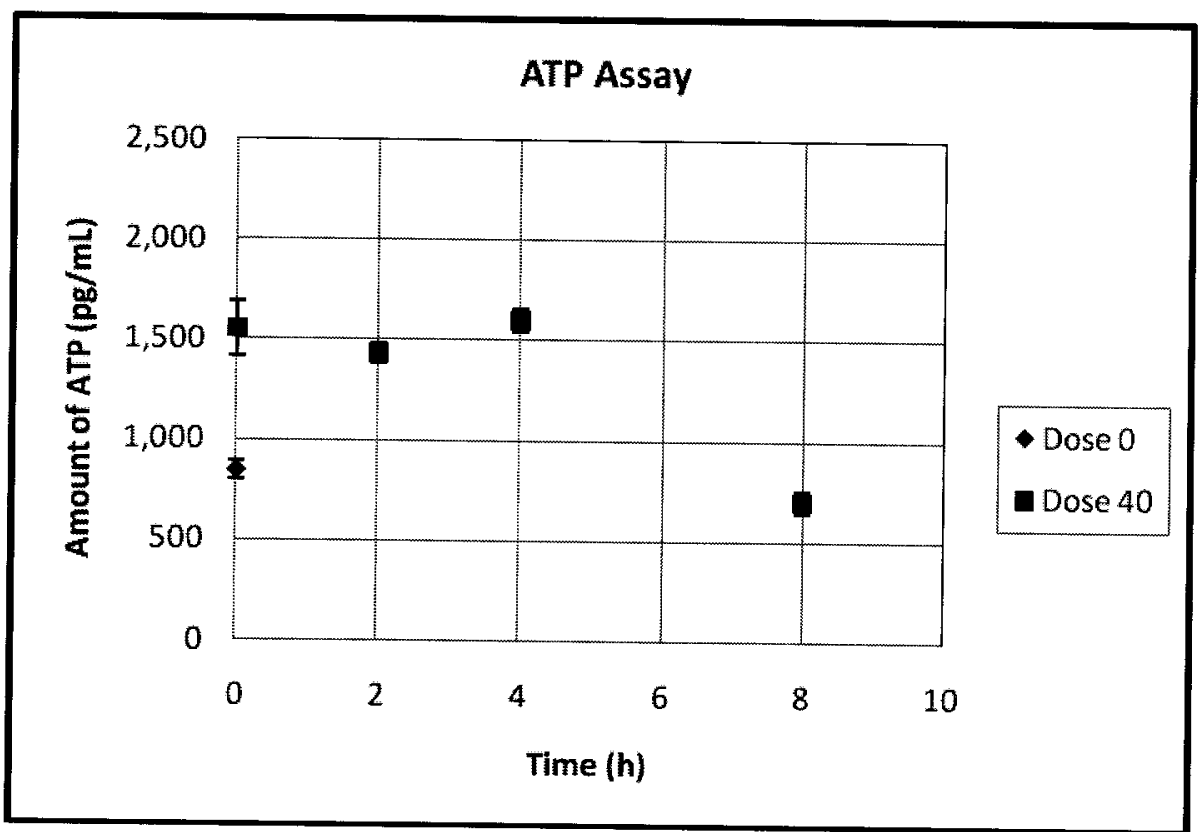

Figure 6.29: Results of ATP assay of a sample with initial bacterial concentration of $1.9 \times 10^{5} \pm$ $3 \times 10^{4} \mathrm{CFU} / \mathrm{mL}$ exposed to $\mathrm{UV}$ radiation of a dose of $40 \mathrm{~mJ} / \mathrm{cm}^{2}$ and incubated at room temperature for 8 hours. 


\subsubsection{EFFECT OF PRETREATMENT}

To improve the sensitivity of the ATP assay to UV disinfection, LuminUltra had performed preliminary tests using various treatment methods to be performed before ATP

analysis. A total of four pretreatment methods were tested, and the fourth pretreatment showed the most potential for success (see Table A. 1). The following set of experiments aimed to assess the effect of this pretreatment method on ATP measurements.

The pretreatment method was designed to first stress the bacterial cells to eliminate ATP from damaged cells and to then stimulate growth of the remaining cells in an effort to cause autolysis of the cells with damaged DNA. Between each step, an incubation period was added to allow for the cells to react. The first incubation period was 30 minutes and the second was 2 hours.

To create the stressful environment, a dilution buffer made of water and trisodium phosphate called LumiSolveX10 was added to the irradiated sample (LuminUltra Technologies Inc. 2009). To create the growth stimulating environment for the cells, yeast extract was added. For further details on the"procedure of the pretreatment method, see Section 5.7.3.3.

An initial sample solution was prepared that had a bacteria concentration of $1.5 \times 10^{5} \pm$ $2 \times 10^{4} \mathrm{CFU} / \mathrm{mL}$, as measured by membrane filtration initial amount of ATP of $886 \pm 51$ $\mathrm{pg} / \mathrm{mL}$ of ATP, as measured by the ATP assay. For this experiment, three UV doses of 0 , 40 and $60 \mathrm{~mJ} / \mathrm{cm}^{2}$ were selected for observation and irradiation was performed following the procedures outlined in Section 5.7.3. After irradiation, the samples were subjected to the forth pretreatment method, as outlined in Section 5.7.3.3 and measured in parallel 
with the membrane filtration (see Section 5.4) and the ATP assay using a sample volume of 5.0mL and Luminase (see Section 5.5).

Parallel results of the membrane filtration and the ATP assay are presented in Table 6.6. The pretreatment method was able to significantly reduce the amount of ATP measured after irradiation. However, the membrane filtration results showed that the pretreatment method resulted in no culturable bacteria even for the sample that received $0 \mathrm{~mJ} / \mathrm{cm}^{2} \mathrm{UV}$ dose. This indicates that the pretreatment method killed the cells in the sample, and the low levels of ATP measured after pretreatment were attributed to the background emission of light.

Table 6.6: Summary of the results of parallel membrane filtration and ATP assays of samples after the application of a pretreatment method.

\begin{tabular}{|c|c|c|c|c|c|}
\hline \multirow{3}{*}{$\begin{array}{l}\text { Pretreatment } \\
\text { Method }\end{array}$} & \multirow{3}{*}{$\begin{array}{l}\text { UV Dose } \\
\left(\mathrm{mJ} / \mathrm{cm}^{2}\right)\end{array}$} & \multirow{2}{*}{\multicolumn{2}{|c|}{$\begin{array}{l}\text { Membrane Filtration } \\
\text { Bacteria Concentration } \\
(\mathrm{CFU} / \mathrm{mL})\end{array}$}} & \multirow{2}{*}{\multicolumn{2}{|c|}{$\begin{array}{l}\text { ATP Assay } \\
\text { Amount of ATP } \\
(\mathrm{pg} / \mathrm{mL})\end{array}$}} \\
\hline & & & & & \\
\hline & & Average & $\begin{array}{l}\text { Standard } \\
\text { Deviation }\end{array}$ & Average & $\begin{array}{l}\text { Standard } \\
\text { Deviation }\end{array}$ \\
\hline None & 0 & $1.5 \times 10^{5}$ & $2 \times 10^{4}$ & 860 & 51 \\
\hline \multirow{3}{*}{$\begin{array}{l}\text { Forth Pretreatment } \\
\text { Method }\end{array}$} & 0 & 0 & & 41 & 1 \\
\hline & 40 & 0 & & 52 & 3 \\
\hline & 60 & 0 & & 59 & 2 \\
\hline
\end{tabular}




\subsection{Phase III: Chlorine Disinfection Experiments}

The purpose of Phase III was to assess the use of the ATP assay as a monitoring tool for chlorine disinfection of water and wastewater. Chlorine, a common disinfection method for water and wastewater, is highly reactive and oxidizes the cellular structures of cells, which can ultimately lead to their death. If the cell membrane is ruptured, ATP is released from the cell and becomes extracellular ATP. In presence of chlorine, this ATP would also be oxidized subsequently. The ATP test is able to separate intracellular ATP from the extracellular ATP using a filtration step. Therefore, intracellular ATP, which belongs to viable cells only, can be measured and used as a monitoring tool for chlorine disinfection.

Initial chlorine disinfection experiments were performed using a stock solution of $1 \mathrm{~g} / \mathrm{L}$ of sodium hypochlorite. Three initial chlorine concentrations of 1,5 and $10 \mathrm{mg} / \mathrm{L} \mathrm{Cl}_{2}$ were investigated at five contact times: $0,5,15,30$ and 45 minutes. The chlorine experiments were performed following the procedure outlined in Section 5.7.4. The bacteria concentration was then measured following the membrane filtration procedure outlined in Section 5.4, and the amount of ATP was measured following the ATP assay procedure outlined in Section 5.5, with $5.0 \mathrm{~mL}$ samples and regular strength Luminase.

The initial sample solutions, which were made with $0.1 \%$ peptone water, had bacteria concentrations of $1.4 \times 10^{5} \pm 2 \times 10^{4} \mathrm{CFU} / \mathrm{mL}, 1.3 \times 10^{5} \pm 5 \times 10^{4} \mathrm{CFU} / \mathrm{mL}$ and $3.0 \times 10^{5} \pm$ $3 \times 10^{4} \mathrm{CFU} / \mathrm{mL}$ for chlorine concentrations of 1,5 and $10 \mathrm{mg} / \mathrm{L}$. The results of the membrane filtration for the chlorine concentrations of 1,5 and $10 \mathrm{mg} / \mathrm{L} \mathrm{Cl}_{2}$ are shown in Figure 6.30, Figure 6.31 and Figure 6.32 respectively, where the $\log$ of the bacteria concentration ( $\mathrm{x}$-axis) is plotted against the contact times (y-axis). 
From the results of the membrane filtration, it would be expected that the bacteria concentration would decrease with both an increase in contact time and chlorine concentration. This trend can be seen in the aforementioned figures, with the most significant decrease in bacteria concentration seen with the highest chlorine concentration of $10 \mathrm{mg} / \mathrm{L}$ where a $5-\log$ removal of bacteria was achieved.

Results of the ATP assay for the chlorine concentrations of 1,5 and $10 \mathrm{mg} / \mathrm{L} \mathrm{Cl}_{2}$ are shown in Figure 6.33, Figure 6.34 and Figure 6.35 respectively, where the amount of ATP (x-axis) is plotted against the contact times (y-axis). If the bacteria cells are fully oxidized to release their contents and the extracellular ATP is removed by filtration, then a similar trend seen in the results of the membrane filtration would be expected here. Results of the $10 \mathrm{mg} / \mathrm{L} \mathrm{Cl}_{2}$ in Figure 6.35 clearly demonstrate this trend. The decrease in the amount of ATP was measured to be approximately half of the initial amount while the results of the membrane filtration showed a 5-log.

There are other elements about the results of the ATP assay to note. To begin with, the initial amounts of ATP are inconsistent. Each experimental solution began with an initial bacteria concentration in the order of $10^{5} \mathrm{CFU} / \mathrm{mL}$. In addition, all experiments were performed on the same day and with the same bacteria stock solution. It would then be expected that the initial concentration of each solution would be the same. From previous experiments, the expected amount of ATP was in the order of $10^{3} \mathrm{pg} / \mathrm{mL}$. The observed initial amounts of ATP were approximately 450, 225 and 1,100 pg/mL, respectively, for chlorine concentrations of 1 and 5 and $10 \mathrm{mg} / \mathrm{L}$, where only the chlorine concentration of $10 \mathrm{mg} / \mathrm{L}$ is in the expected range. The use of larger sample volumes as well as Luminase XXL can be used to improve the sensitivity limits of the test (see Phase I in Section 6.2). 
The chlorine experiments were repeated using larger samples sizes and the stronger Luminase reagent.

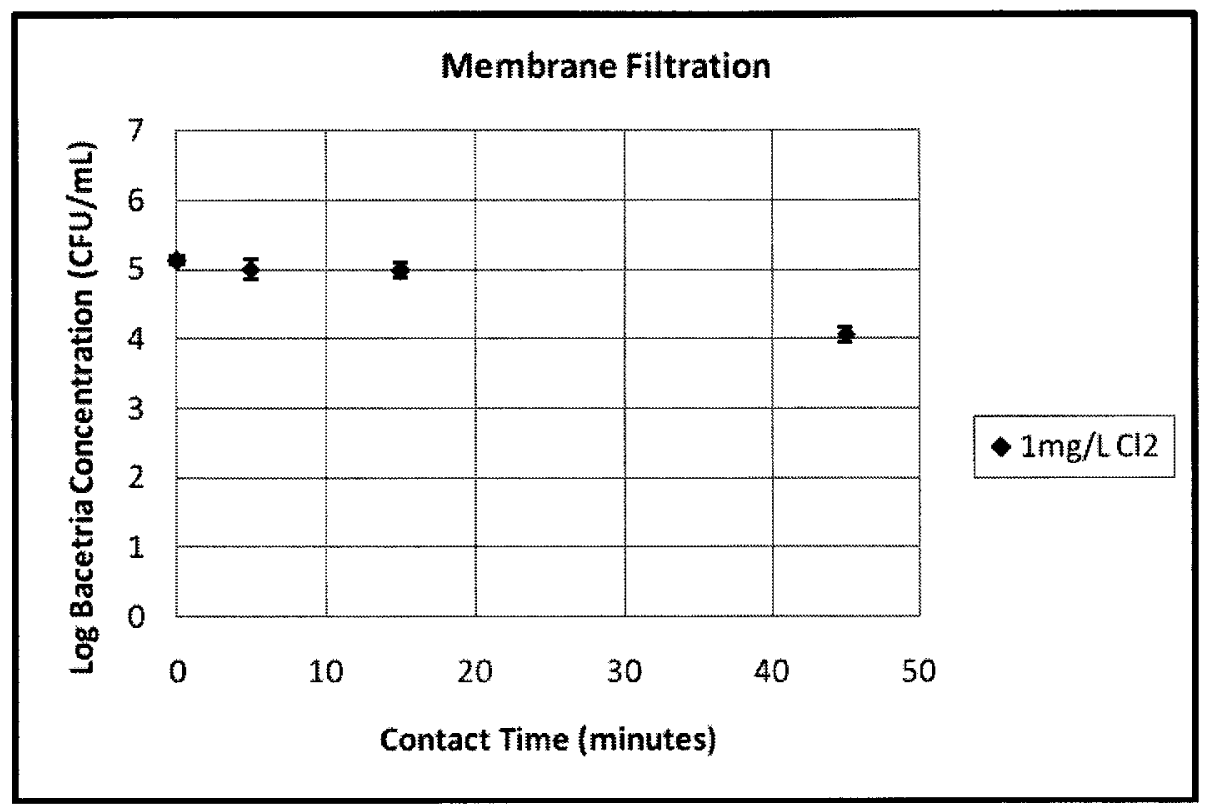

Figure 6.30: Results of membrane filtration of a sample with initial bacteria concentration of $1.4 \times 10^{5} \pm 2 \times 10^{4} \mathrm{CFU} / \mathrm{mL}$ and exposed to an initial chlorine concentration of $0.72 \mathrm{mg} / \mathrm{L} \mathrm{Cl}_{2}$ for up to 45 minutes. 


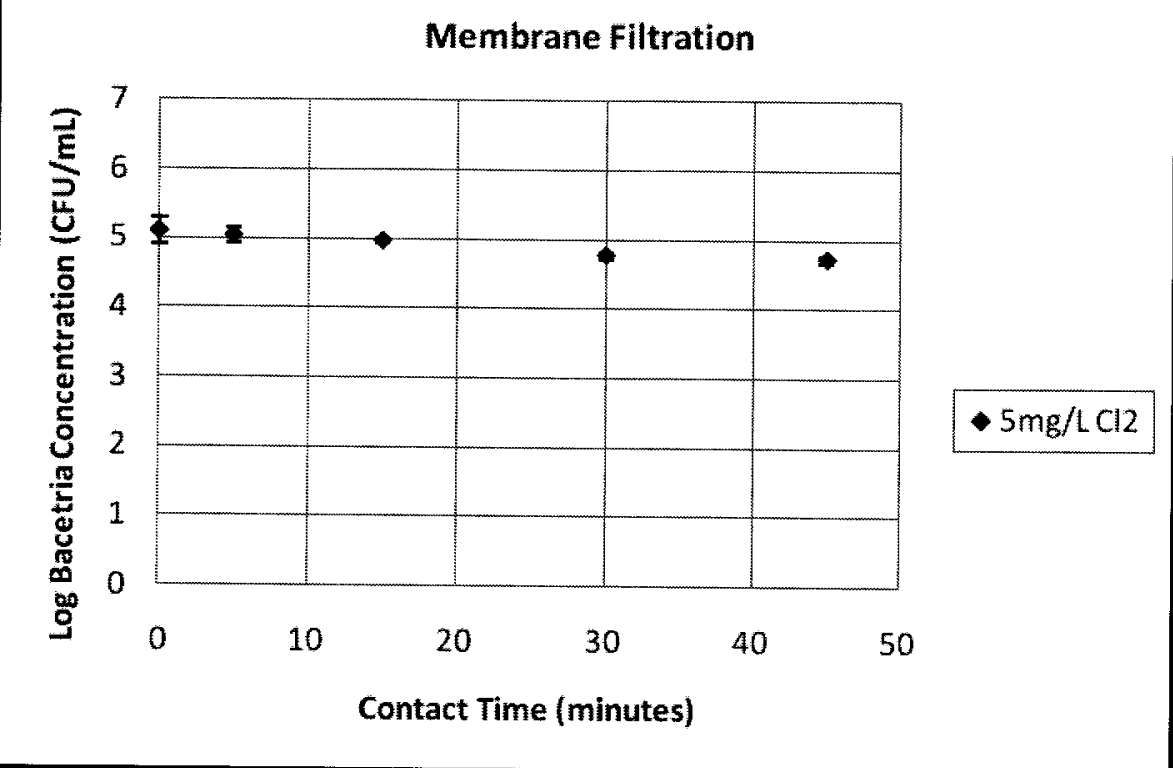

Figure 6.31: Results of membrane filtration of a sample with initial bacteria concentration of $1.3 \times 10^{5} \pm 5 \times 10^{4} \mathrm{CFU} / \mathrm{mL}$ and exposed to an initial chlorine concentration of $5.10 \mathrm{mg} / \mathrm{L} \mathrm{Cl}$ for up to 45 minutes.

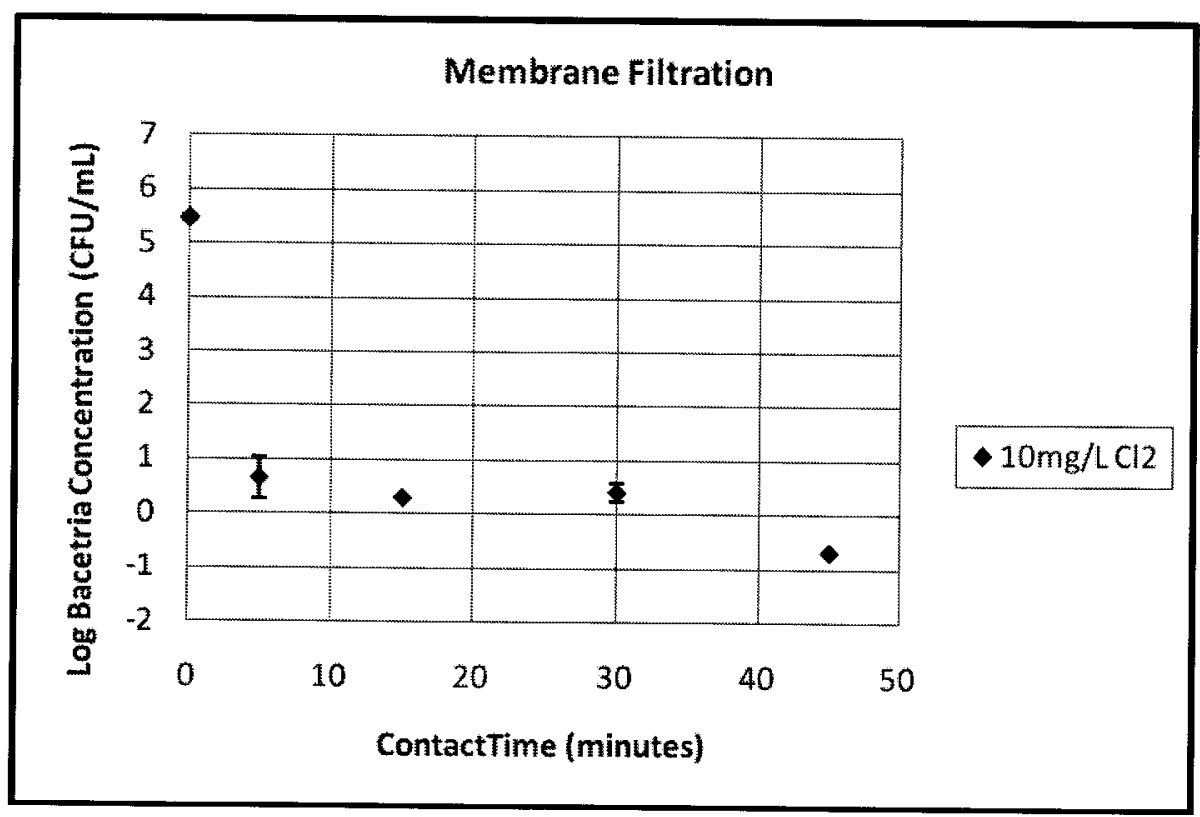

Figure 6.32: Results of membrane filtration of a sample with initial bacteria concentration of $3.0 \times 10^{5} \pm 3 \times 10^{4} \mathrm{CFU} / \mathrm{mL}$ and exposed to an initial chlorine concentration of $11.00 \mathrm{mg} / \mathrm{L} \mathrm{Cl}$ for up to 45 minutes. 


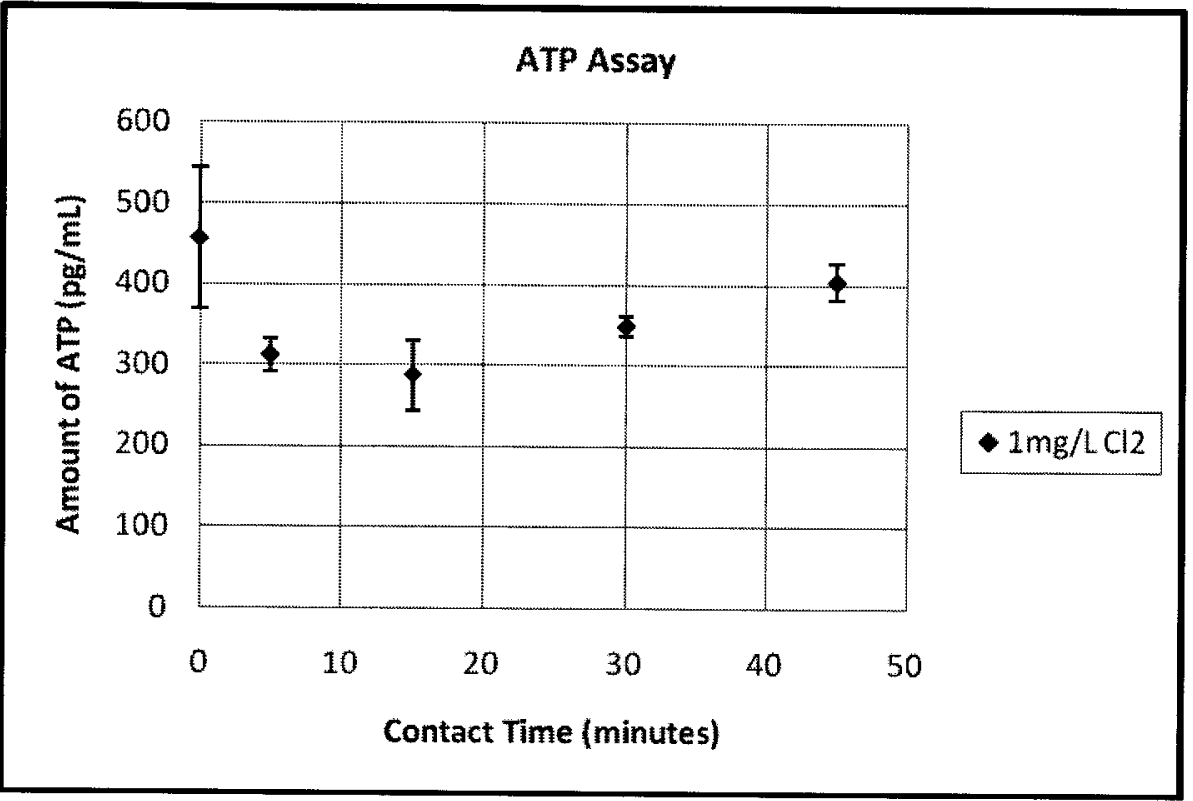

Figure 6.33: Results of ATP assay of a sample with initial bacteria concentration of $1.4 \times 10^{5} \pm 2 \times 10^{4} \mathrm{CFU} / \mathrm{mL}$ and exposed to an initial chlorine concentration of $0.72 \mathrm{mg} / \mathrm{L} \mathrm{Cl}_{2}$ for up to 45 minutes.

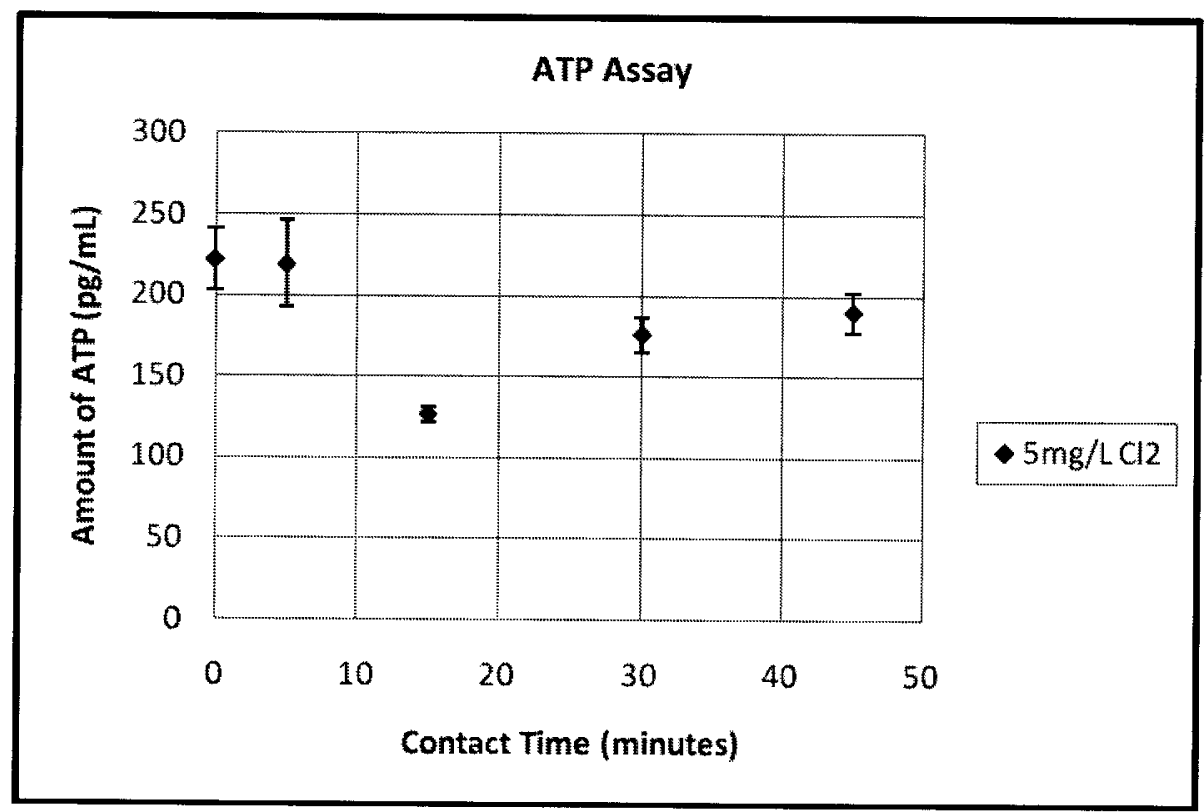

Figure 6.34: Results of ATP assay of a sample with initial bacteria concentration of $1.3 \times 10^{5} \pm 5 \times 10^{4} \mathrm{CFU} / \mathrm{mL}$ and exposed to an initial chlorine concentration of $5.10 \mathrm{mg} / \mathrm{L} \mathrm{Cl}_{2}$ for up to 45 minutes. 


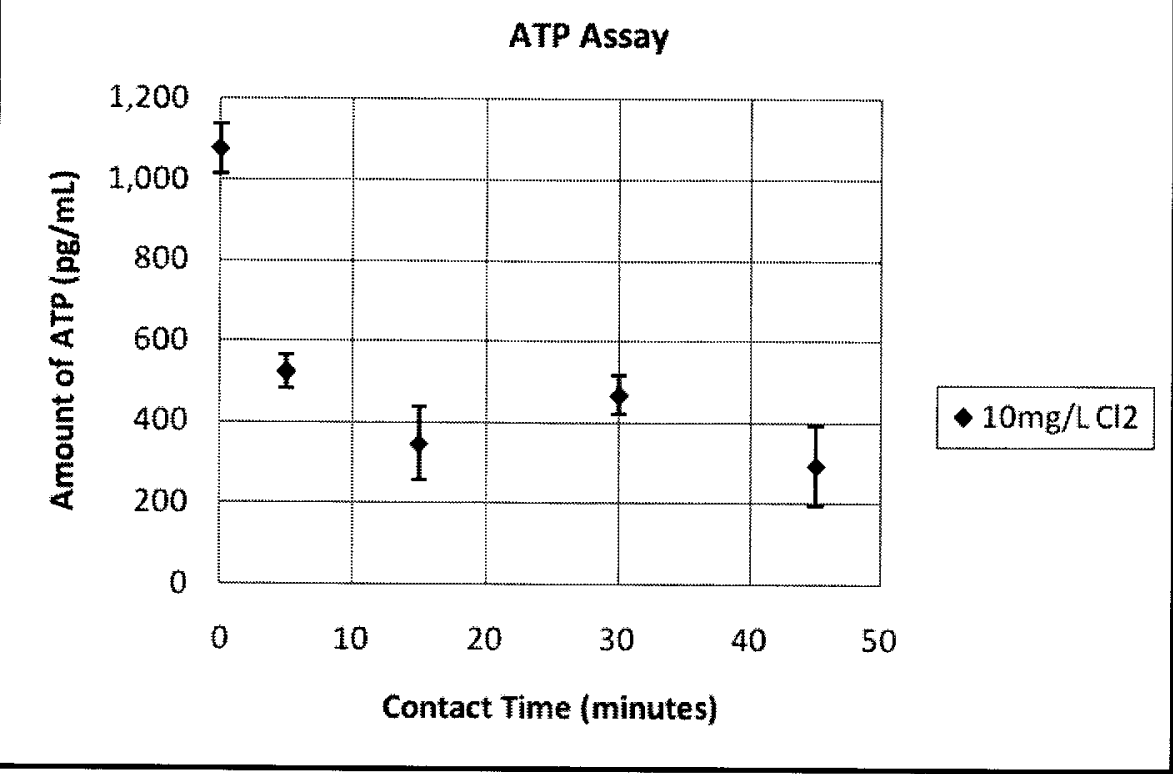

Figure 6.35: Results of ATP assay of a sample with initial bacteria concentration of $3.0 \times 10^{5} \pm 3 \times 10^{4}$ $\mathrm{CFU} / \mathrm{mL}$ and exposed to an initial chlorine concentration of $11.00 \mathrm{mg} / \mathrm{L} \mathrm{Cl}_{2}$ for up to 45 minutes.

As mentioned, the chlorine experiments were repeated with larger sample volume of $100.0 \mathrm{~mL}$, a XXL Luminase. The base solution used was also changed from $0.1 \%$ peptone to PBS. Unlike PBS, the peptone solution used consumed chlorine. For this set of experiments, the chlorine concentrations were reduced to $0.9,0.6$ and $0.4 \mathrm{mg} / \mathrm{L} \mathrm{Cl}_{2}$ with contact times of $0,1,5,10$ and 15 minutes.

Results of the membrane filtration are shown in Figure 6.36 to Figure 6.38. For chlorine concentration of $0.9 \mathrm{mg} / \mathrm{L} \mathrm{Cl} \mathrm{Cl}_{2}$ all contact times showed no bacteria growth. The corresponding figure, Figure 6.36 , shows the initial bacteria concentration of $2.0 \times 10^{5} \pm$ $5 \times 10^{4} \mathrm{CFU} / \mathrm{mL}$ followed by a rapid drop to bacteria counts of $0 \mathrm{CFU} / \mathrm{mL}$. For this figure contact time has been plotted against bacteria concentration (y-axis). For the other chlorine trials, contact time (x-axis) was plotted against log of the bacteria concentration (y-axis). For the sample with initial chlorine concentration of $0.6 \mathrm{mg} / \mathrm{L} \mathrm{Cl}_{2}$, initial E.coli bacteria concentration was determined to be $1.6 \times 10^{5} \pm 2 \times 10^{4} \mathrm{CFU} / \mathrm{mL}$. With increasing 
contact times the bacteria concentration was reduced to a final count of $0 \mathrm{CFU} / \mathrm{mL}$ at a 10 minute contact time which represents a 5-log removal of bacteria (Figure 6.37). A similar trend was observed for the chlorine concentration of $0.4 \mathrm{mg} / \mathrm{L} \mathrm{Cl}_{2}$ which had an initial bacteria concentration of $1.5 \times 10^{5} \pm 2 \times 10^{4} \mathrm{CFU} / \mathrm{mL}$ and a $4.5-\log$ removal was achieved at 15 minutes (Figure 6.38).

Results of the ATP assay are shown in Figure 6.39 to Figure 6.41 where amount of ATP (y-axis) has been plotted against contact time (x-axis). The initial amount of ATP for the sample exposed to a chlorine concentration of $0.9 \mathrm{mg} / \mathrm{L} \mathrm{Cl}_{2}$ was $60.0 \pm 12.23 \mathrm{pg} / \mathrm{mL}$, which quickly dropped to quantities below $1 \mathrm{pg} / \mathrm{mL}$ for contact times greater than 1 minute (Figure 6.39). Similar, but slightly more gradual trends were observed for the sample with chlorine concentrations of 0.6 and $0.4 \mathrm{mg} / \mathrm{L} \mathrm{Cl}_{2}$. The initial quantity of ATP for the sample of $0.6 \mathrm{mg} / \mathrm{L} \mathrm{Cl}_{2}$ was $56.56 \pm 3.41 \mathrm{pg} / \mathrm{mL}$, which was decreased to $2.5 \pm$ $0.89 \mathrm{pg} / \mathrm{mL}$ at 1 minute and further decreased to less than $1 \mathrm{pg} / \mathrm{mL}$ thereafter (Figure 6.40). The sample with chlorine concentration of $0.4 \mathrm{mg} / \mathrm{L} \mathrm{Cl}_{2}$ had an initial quantity of ATP of $75.88 \pm 6.49 \mathrm{pg} / \mathrm{mL}$, which was decreased to $5.99 \pm 0.67 \mathrm{pg} / \mathrm{mL}$ at 1 minute, further decreased to $2.25 \pm 0.05 \mathrm{pg} / \mathrm{mL}$ at 5 minutes and levelled off at $1.82 \pm 0.53 \mathrm{pg} / \mathrm{mL}$ and $1.80 \pm 0.27 \mathrm{pg} / \mathrm{mL}$ at 10 and 15 minutes respectfully (Figure 6.41).

Both results of the ATP assay and the membrane filtration followed a similar trend. The quantities of ATP and bacteria concentrations began high and decreased with the addition of chlorine. Results of the membrane filtration showed reduction in the bacteria counts between 4 and $5 \log$ while the reduction in the quantities of ATP showed at most a 2-log reduction. The membrane filtration results of the $0.9 \mathrm{mg} / \mathrm{L} \mathrm{Cl}_{2}$ sample showed no bacteria colonies after a contact time of 1 minute. Comparing the corresponding results of the 
ATP assay, there was a minimum of $0.44 \mathrm{pg} / \mathrm{mL}$ of ATP measured while the amount of ATP in a sample of PBS was measured to be $0.010 \pm 0.008 \mathrm{pg} / \mathrm{mL}$. If all cells have been oxidized by chlorine to release the cellular ATP, then the expected results would be similar to the PBS sample. However, since this is not the case, it is believed that there may be cells that while damaged by chlorine and are unable to replicate and may still contain cellular ATP.

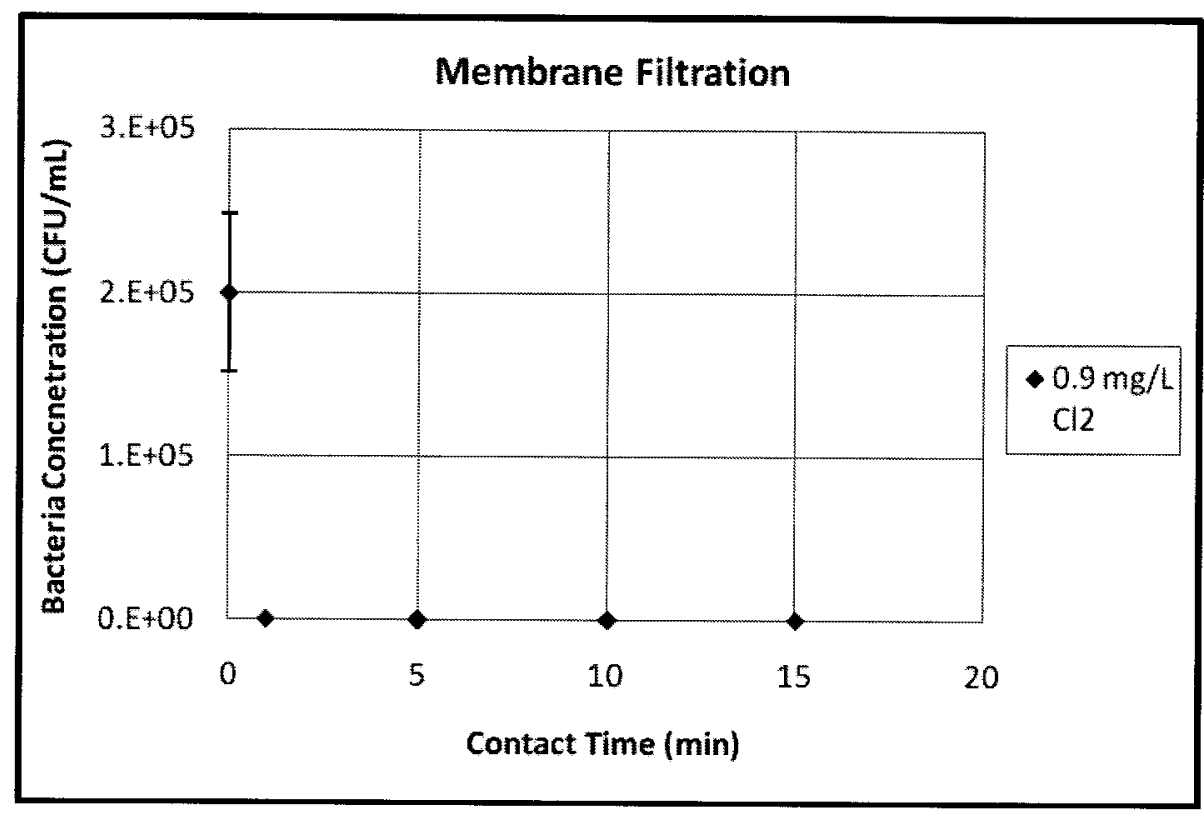

Figure 6.36: Results of membrane filtration of a sample with initial bacteria concentration of $2.0 \times 10^{5}$ $\pm 5 \times 10^{4} \mathrm{CFU} / \mathrm{mL}$ and exposed to an initial chlorine concentration of $0.94 \mathrm{mg} / \mathrm{L} \mathrm{Cl}_{2}$ for 15 minutes. 


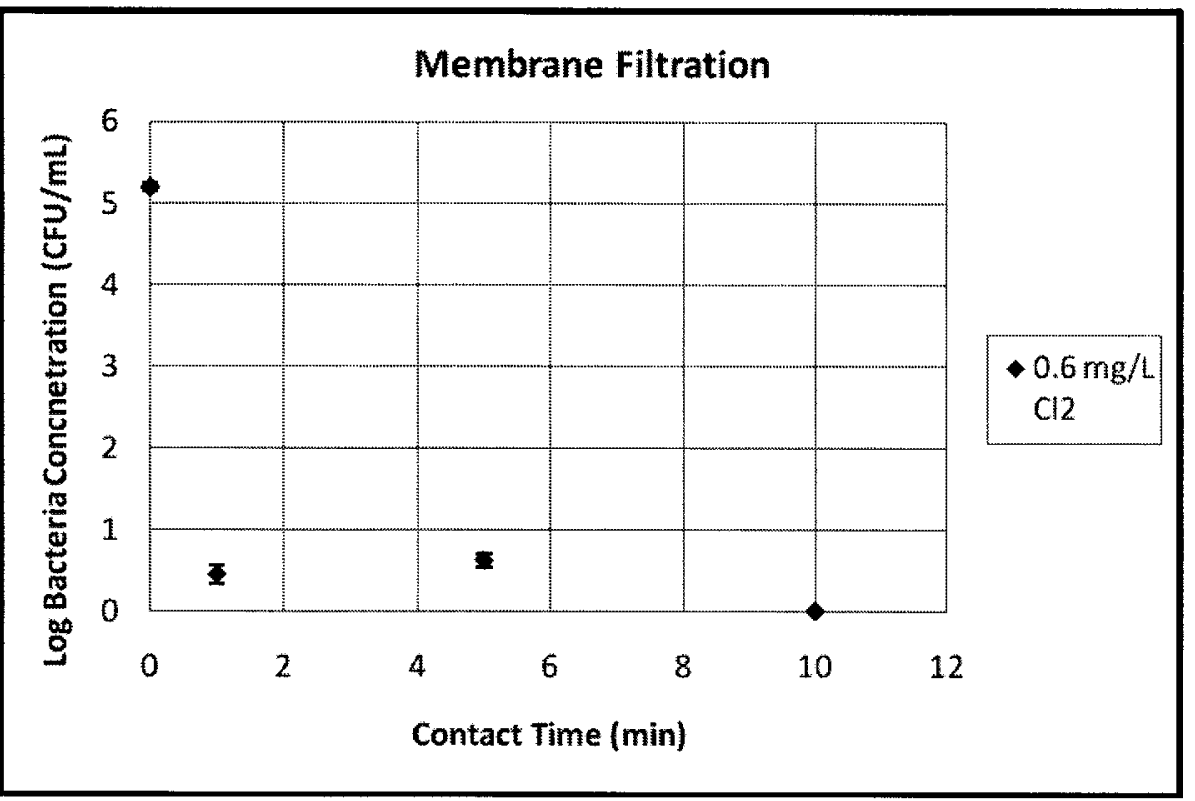

Figure 6.37: Results of membrane filtration of a sample with initial bacteria concentration of $1.6 \times 10^{5}$ $\pm 2 \times 10^{4} \mathrm{CFU} / \mathrm{mL}$ and exposed to an initial chlorine concentration of $0.56 \mathrm{mg} / \mathrm{L} \mathrm{Cl} \mathrm{l}_{2}$ for 10 minutes.

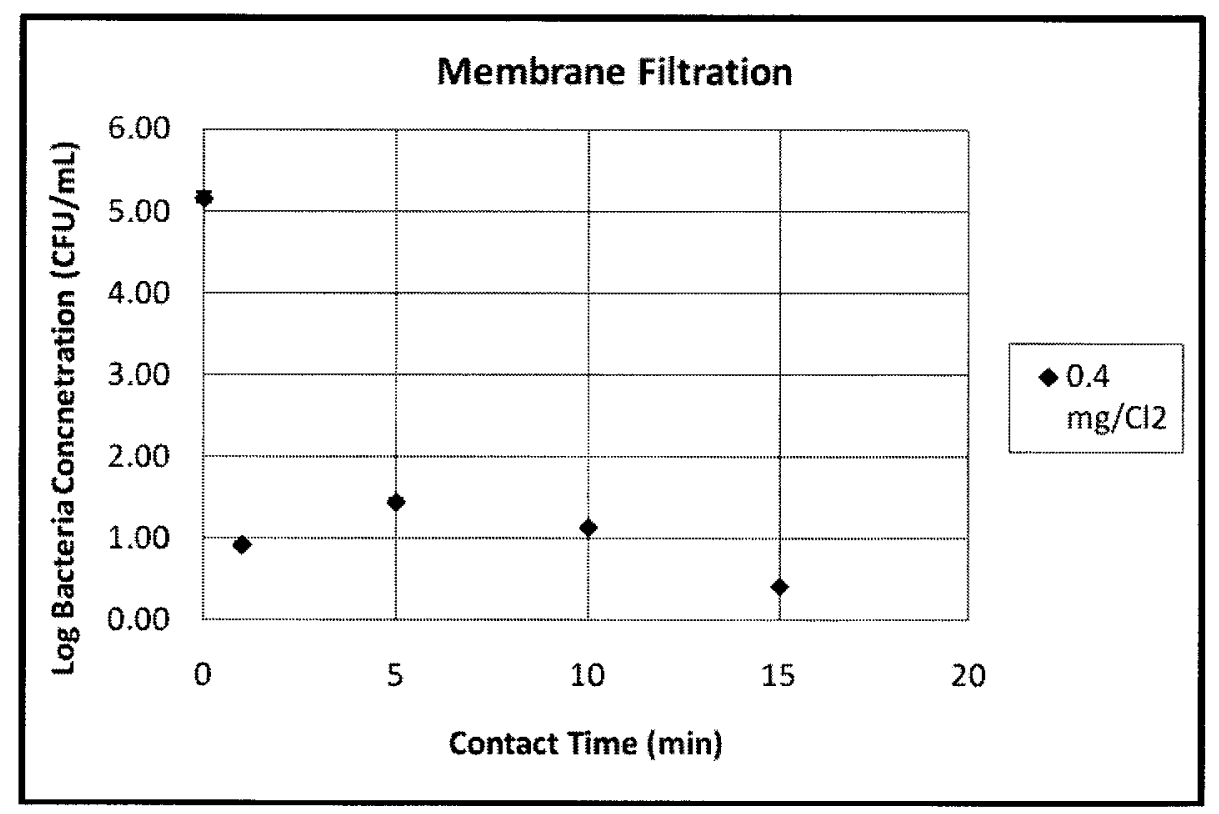

Figure 6.38: Results of membrane filtration of a sample with initial bacteria concentration of $1.5 \times 10^{5}$ $\pm 2 \times 10^{4} \mathrm{CFU} / \mathrm{mL}$ and exposed to an initial chlorine concentration of $0.38 \mathrm{mg} / \mathrm{L} \mathrm{Cl}$ for 15 minutes. 


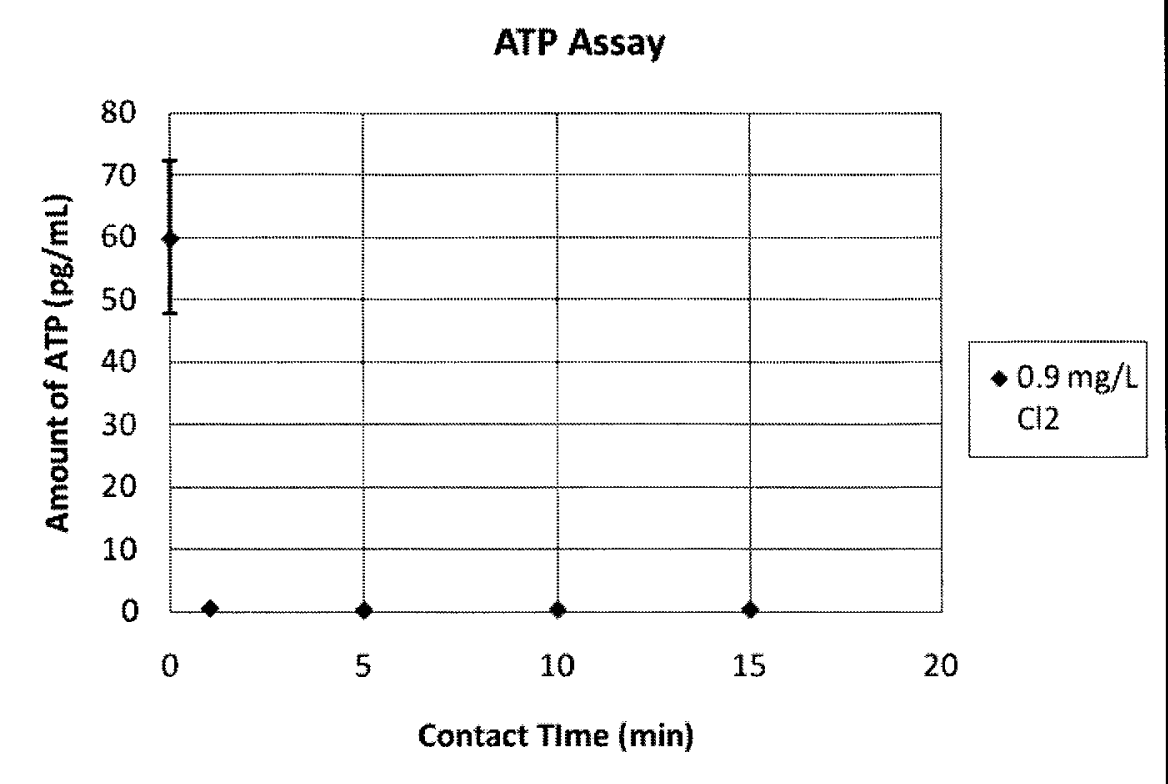

Figure 6.39: Results of the ATP assay using XXL Luminase and $100 \mathrm{~mL}$ volumes of a sample with initial bacteria concentration of $2.0 \times 10^{5} \pm 5 \times 10^{4} \mathrm{CFU} / \mathrm{mL}$ and exposed to an initial chlorine concentration of $0.94 \mathrm{mg} / \mathrm{L} \mathrm{Cl}_{2}$ for 15 minutes.

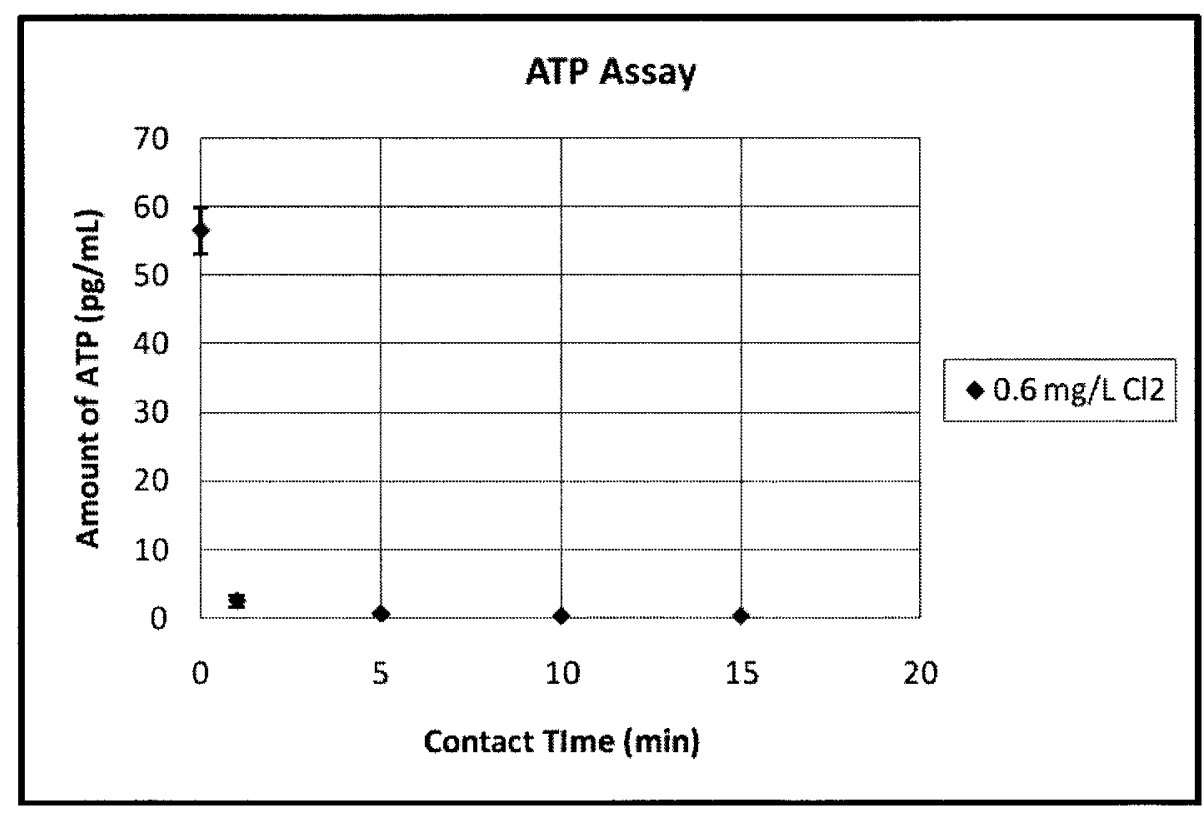

Figure 6.40: Results of the ATP assay using XXL Luminase and $100 \mathrm{~mL}$ volumes of a sample with initial bacteria concentration of $1.6 \times 10^{5} \pm 2 \times 10^{4} \mathrm{CFU} / \mathrm{mL}$ and exposed to an initial chlorine concentration of $0.56 \mathrm{mg} / \mathrm{L} \mathrm{Cl}{ }_{2}$ for 15 minutes. 


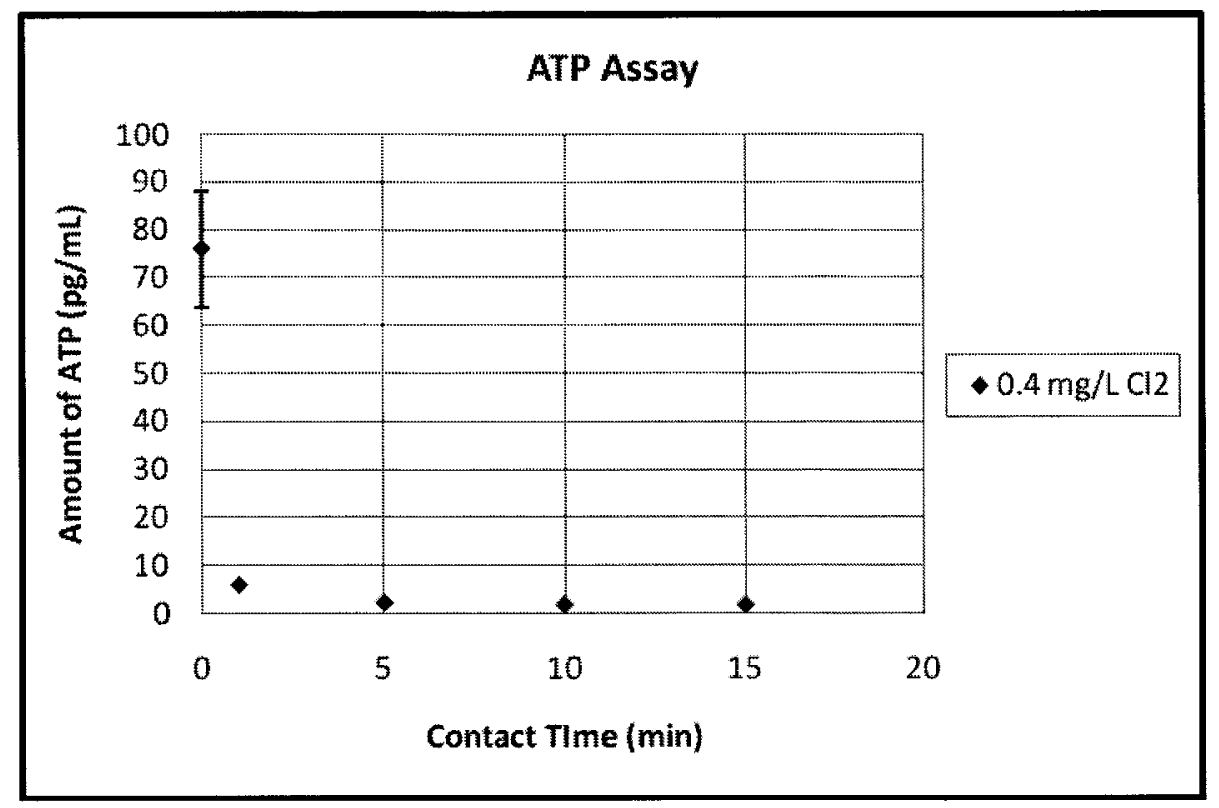

Figure 6.41: Results of the ATP assay using XXL Luminase and $100 \mathrm{~mL}$ volumes of $1.5 \times 10^{5} \pm 2 \times 10^{4}$ $\mathrm{CFU} / \mathrm{mL}$ and exposed to an initial chlorine concentration of $0.38 \mathrm{mg} / \mathrm{L} \mathrm{Cl} \mathrm{Cl}_{2}$ for 15 minutes. 


\section{CHAPTER 7}

\section{SuMMARY AND CONCLUSIONS}

This first objective of this study was to provide an overview of the real-time monitoring tools that are currently available on the market and that are in development with particular attention being paid to Canadian companies and researchers. From the review, it was discovered that chemical monitoring techniques being pursued are primarily concerned with the determination of low concentrations of pharmaceuticals and personnel care products (PPCP). These methods and techniques require complex laboratory procedures and equipment that are not currently suitable for real-time monitoring purposes. Most interestingly current real-time monitoring techniques examined the strategic screening of water using existing probes and the response of living organism (fish, bivalves and daphnia), or even of cells to a contaminant.. Light scattering technologies, such as UVVIS spectroscopy, UV absorbance and fluorescence spectroscopy, have been successfully used to measure general water quality parameters such as total suspended solids (TSS), biochemical oxygen demand (BOD) and dissolved organic matter (DOM). The miniaturization of microbiological contaminant monitoring includes the use of lab-onchip technology, immunoassays and microarrays. However, the working principle of these methods relies on the detection of the DNA of microbiological cells which may include non-viable cells. On the other hand, the ATP molecule quickly decomposes after the death of the cell and thus the ATP assay, which relies on the detection of the ATP molecule, is better able to detect viable microorganism. 
The second objective of this study was to select and evaluate the use of a reviewed technology as a real-time monitoring tool. The ATP assay, more specifically the Quench Gone Aqueous ATP assay by LuminUltra Technologies (Fredericton, NB), was selected for further investigation based on its ease of use, low sensitivity and ability to quantify the viable microbial population of a sample. Throughout the testing of the ATP assay, bacteria counts were compared to the quantities of ATP by use of the membrane filtration techniques and the ATP assay, respectfully. The test was completed in four phase:

- Preliminary Phase: Quality Control and Assurance

- Phase I: Optimization of the ATP assay

- Phase II: UV Experiments

- Phase III: Chlorine Disinfection Experiments

During the preliminary phase, the background levels of the levels of materials and regents needed to complete the test were assessed to be minimal and the results of the ATP assay were determined to be highly reproducible. The ATP assay also showed consistent results for a surface water sample with increased turbidity ranging from $2.56 \pm 0.1$ NTU to 32.43 $\pm 0.55 \mathrm{NTU}$ which suggest the test is suitable for surface water applications. Initial calibration curves were attempted and used sample volumes of $5 \mathrm{~mL}$ and regular Luminase to complete the ATP assay. While the sample volume used was at the lower end of the suggested range (5 to $100 \mathrm{~mL}$ ), the lower sensitivity limit of the assay was determined to be in the order of $10^{5} \mathrm{CFU} / \mathrm{mL}$ which was much lower than expected. For high bacteria concentrations, ranging from $10^{5}$ to $10^{9} \mathrm{CFU} / \mathrm{mL}$, the ATP assay was found to be linear $\left(\mathrm{R}^{2}=0.98\right)$. To establish this curve, the sample volume of the ATP assay was increased to $50 \mathrm{~mL}$ and only decreased if the limits of the luminometer were reached. For 
high bacteria concentrations (between $10^{5}$ and $10^{9} \mathrm{CFU} / \mathrm{mL}$ ) it is recommended that recommended sample volumes to be between 1 and $50 \mathrm{~mL}$ and that sample volumes of 50 $\mathrm{mL}$ should be first attempted and only reduced if the limits of the luminometer have been reached.

During Phase I of the study, varying the pore size of the filtered did not show a significant increase in the amount of ATP measured and increased the difficulty of filtering any meaningful amounts of sample volume. The amount of lysing solution was also increased in an effort to improve the performance of the test; however, the attempt was unsuccessful. To improve the detection limits of the test at lower bacteria counts, the strength of the Luminase reagent was increased and XXL Luminase was employed to complete the assay. In addition, sample volumes were increased to a maximum of $1 \mathrm{~L}$. With this change in testing procedures, the sensitivity limit of the assay was successfully increased to $10^{2} \mathrm{CFU} / \mathrm{mL}$. Thus, for samples with low bacteria concentrations (between $10^{2}$ and $10^{5} \mathrm{CFU} / \mathrm{mL}$ ), it is recommended that the ATP assay be used with XXL Luminase and sample volumes of up to $1 \mathrm{~L}$ where sample volumes of $1 \mathrm{~L}$ should first be attempted and only reduced if the limits of the luminometer are reached. It is also important to note that comparisons between the bacteria counts (confirmed by membrane filtration) and the quantities of ATP (measured by the QGA assay) have never before been attempted. The sensitivity limit of $0.1 \mathrm{pg} /$ was not achieved. The sensitivity limits achieved in this study were between 0.5 and $1 \mathrm{pg} / \mathrm{mL}$. 
UV irradiation performed with low pressure UV lamps did not affect the amount of ATP measured before and after UV irradiation and initial experiments indicated that the ATP assay was not suitable as a monitoring or system optimization tool for UV disinfection. Attempts were made to improve the performance of the ATP assay with the introduction of an incubation period at different temperatures. However, these attempts did not improve the performance of the ATP assay. A pretreatment method was also tested and was found to reduce the amount of ATP after irradiation; however, it did so erroneously. The pretreatment method increased the $\mathrm{pH}$ of the irradiated sample to the point that it lysed bacteria. Once compared to the results of the membrane filtration, it was clear that the pretreatment method resulted in cell death.

The final experimental phase tested the ATP assay for its use with chlorinated samples. Unlike UV irradiation, which inactivates the cells to inhibit replication through damage to DNA, chlorine disinfection is a chemical process that oxidizes cell components including cellular ATP. There were similar trends observed between the quantity of bacteria and ATP, however further testing using larger sample volumes is required to confirm this relationship and fully assess the use of the ATP assay with chlorinated samples. 
The overall conclusions of this study are summarized as follows:

- Subsequent to a review of current and emerging technologies that more quickly evaluate water quality parameters, the ATP assay was selected for further study for its ability to measure not only cellular ATP but also the viability of cells.

- The initial sensitivity limit of the ATP assay of $10^{5} \mathrm{CFU} / \mathrm{mL}$ was improved to $10^{2}$ $\mathrm{CFU} / \mathrm{mL}$ by increasing the sample volume from $5 \mathrm{~mL}$ to a maximum of $1 \mathrm{~L}$.

- As the turbidity of a sample of surface water increased, the results of the ATP assay remained consistent making the ATP assay suitable for use with surface water.

- The cellular ATP of a sample before and after UV irradiation remained the same and it was determined that the ATP assay was not suitable for use with UV disinfected (with low pressure lamps) water.

- A similar trend in the quantities of ATP and bacteria concentrations were observed before and after chlorination and it is believed that with further improvements the ATP assay would be a suitable tool for chlorinated water. 


\section{CHAPTER 8}

\section{RECOMMENDATIONS FOR FUTURE STUDIES}

Based on the conclusions drawn in the previous section the following recommendations for further study are suggested:

- The sensitivity limit of the assay may be improved with the increased sensitivity of the luminometer. Similar studies can be conducted comparing the limits of the current instrument to those of instruments from different companies. Improvements to the current luminometer can also be investigated.

- For practicality, the use of a pump to filter larger sample volumes should be investigated. If successful a pump could be more easily deployed for field applications such as for monitoring surface water or effluent wastewater.

- To further investigate the use of the ATP assay as monitoring to for chlorinated samples, the chlorine experiments can be repeated with larger sample volumes.

- Investigate the effect of medium pressure UV lamps on the quantity of ATP before and after UV irradiation. Medium pressure lamps may have more of an effect on the activity of cells and thus have a more measureable effect on the quantities of ATP. 
- In conjunction with molecular identification techniques, such as magnetic beads, future studies may also aim to identify the source of the microbial ATP (i.e. type of bacteria, viruses, algae, etc.). 


\section{REFERENCES}

Adams, John a., and David Mccarty. 2007. Real-Time, on-Line Monitoring of Drinking Water for Waterborne Pathogen Contamination Warning. International Journal of High Speed Electronics and Systems 17, no. 04: 643. doi:10.1142/S0129156407004850.

Addinsoft. 2010. XLSTAT. New York, NY: Addinsolft. www.xlstat.com.

Ahmad, S.R., and D.M. Reynolds. 1999. Monitoring of water quality using fluorescence technique: prospect of on-line process control. Water Research 33, no. 9: 2069-2074.

Allen, Jeannie. 2001. Ultraviolet Radiation: How it Affects Life on Earth. Earth Observatory. earthobservatory.nasa.gov/.

Allis, O, J Dauphard, B Hamilton, A Ni Shuilleabhain, M Lehane, J.K James, and A Furey. 2007. Liquid chromatography tandam mass spectrometry application, for the determination of extracellular hepatotoxins in Irish lake and drinking waters. Analytical Chemistry 79, no. 9: 3436-3447.

American Public Health Association (APHA), American Water Works Association (AWWA), and Water Environment Federation (WEF). 2005. Standard Methods for the Examination of Water and Wastewater. Andrew D. Eaton, Lenore S. Clesceri, Eugene W. Rice, and Arnold E. Greenberg. 21 ed. Washington, DC: APHA, AWWA, WEF.

Arnon, S. S., R Schechter, T.V. Inglesby, D.A. Henderson, J.G. Bartlett, M.S. Ascher, E. Eitzen, et al. 2001. Botulinum Toxin as a Biological Weapon: Medical and Public Health Management. The Journal of the American Medical Association 285, no. 8: 1059-1070.

Behets, Jonas, Priscilla Declerck, Yasmine Delaedt, Lieve Verelst, and Frans Ollevier. 2007. A duplex real-time PCR assay for the quantitative detection of Naegleria fowleri in water samples. Water research 41, no. 1: 118-26.

Benett, William J. 2000. Handheld advanced nucleic acid analyzer. Proceedings of SPIE 4200: 55-63.

Berney, Michael, Hans-Ulrich Weilenmann, and Thomas Egli. 2006. Flow-cytometric study of vital cellular functions in Escherichia coli during solar disinfection (SODIS). Microbiology (Reading, England) 152, no. Pt 6: 1719-29.

Bhattacharyya, Jessica, David Read, Sean Amos, Stephen Dooley, Kenneth Killham, and Graeme I Paton. 2005. Biosensor-based diagnostics of contaminated groundwater: assessment and remediation strategy. Environmental pollution (Barking, Essex : 1987) 134, no. 3: 485-92. 
Bolton, J R, and K G Linden. 2003. Standardization of methods for fluence (UV dose) determination in bench-scale UV experiments. Journal of Environmental Engineering 129, no. 3: 209-215.

Bonastre, a, R Ors, J Capella, M Fabra, and M Peris. 2005. In-line chemical analysis of wastewater: present and future trends. TrAC Trends in Analytical Chemistry 24, no. 2: 128-137.

Bourgeois, Wilfrid, Joanna E Burgess, and Richard M Stuetz. 2001. On-line monitoring of wastewater quality: a review. Journal of Chemical Technology \& Biotechnology 76, no. 4: 337-348.

Brousseau, R. 2004. Application of DNA Microarray Technology for Wastewater Analysis. London, UK.

Bushon, Rebecca N, Christina A Likirdopulos, and Amie M G Brady. 2009. Comparison of immunomagnetic separation/adenosine triphosphate rapid method to traditional culture-based method for E. coli and enterococci enumeration in wastewater. Water research 43, no. 19: 4940-6.

Canadian Council Of Ministers of the Environment (CCME), and Federal-ProvincialTerritorial Committee On Environmental And Occupational Health. 2002. From Source to Tap: The multi-barrier approach to safe drinking water. Source.

Canadian Council Of Ministers of the Environment (CCME), and Federal-ProvincialTerritorial Committee On Environmental And Occupational Health. 2002. From Source to Tap: The multi-barrier approach to safe drinking water. Source.

Casson, L.W, States S.J, J Wichterman, and A Zimmerman. 2006. Thwarting terrorists. Water Environment Laboratory Solutions 13, no. 1: 1-8.

Chang, K. 2003. Refining sensors for bio-terror attacks. International Herald Tribune, Health and Science Section.

Chesnot, T, X Marly, S Chevalier, O Estévenon, M Buès, and J Schwartzbrod. 2002. Optimised immunofluorescence procedure for enumeration of Cryptosporidium parvum oocyst suspensions. Water research 36, no. 13: 3283-8.

Cho, Min, and Jeyong Yoon. 2007. The application of bioluminescence assay with culturing for evaluating quantitative disinfection performance. Water research 41 , no. 4: 741-6.

Colilla, Montserrat, Carlos J. Fernandez, and Eduardo Ruiz-Hitzky. 2002. Case-based reasoning (CBR) for multicomponent analysis using sensor arrays: Application to water quality evaluation. Analyst 127, no. 12: 1580-1582. 
Colwell, R.R. 2000. Viable but nonculturable bacteira: a survival strategy. Journal of Infection and Chemotherapy 6, no. 2: 121-125.

Currell, Graham, and Antony Dowman. 2005. Essential Mathematics and Statistics for Science. Etobicoke, Ontario: John Wiley \& Sons, Ltd.

Deininger, R A, and J Lee. 2001. Rapid determination of bacteria in drinking water using an ATP assay. Field Analytica Chemistry and Technology 5, no. 4: 185-189.

Delahaye, E, B Welte, Y Levi, G Leblon, and A Montiel. 2003. An ATP-based method for monitoring the microbiological drinking water quality in a distribution network. Water Research 37, no. 15: 3689-3696.

Denburg, J.L., and W.D. McElroy. 1970. Anion inhibition of firefly luciferase. Biochemistry and Biophysics 141, no. 2: 668-675.

Dennis, Philip, Elizabeth A Edwards, Steven N Liss, and Roberta Fulthorpe. 2003. Monitoring gene expression in mixed microbial communities by using DNA microarrays. Applied and Environmental Microbiology 69, no. 2: 769-778.

Dennison, M.J, and P.F Turner. 1995. Biosensors for Environmental Monitoring. Biotechnology Advances 13, no. 94: 1-12.

Dhar, R.K, Zheng Y, J Rubenstone, and A van Green. 2004. A rapid calorimetric method for measuring arsenic concentrations in groundwater. Analytica Chemica Acta 526: 203209.

Donaldson, K a, D W Griffin, and J H Paul. 2002. Detection, quantitation and identification of enteroviruses from surface waters and sponge tissue from the Florida Keys using real-time RT-PCR. Water research 36, no. 10: 2505-14.

Dorigo, Ursula, Laurence Volatier, and Jean-François Humbert. 2005. Molecular approaches to the assessment of biodiversity in aquatic microbial communities. Water research 39, no. 11: 2207-18.

Dybko, A, W Wroblewski, E Rozniecka, K Pozniakb, J Maciejewski, R Romaniuk, and Z Brzozka. 1998. Assessment of water quality based on multiparameter fiber optic probe. Sensors and Actuators B: Chemical 51, no. 1-3: 208-213.

Farré, Marinella, Lina Kantiani, Sandra Pérez, and Damià Barceló. 2009. Sensors and biosensors in support of EU Directives. Trends in Analytical Chemistry 28, no. 2: 170185.

Ferrari, B., G. Vesey, C. Weir, K.L. Williams, and D.A. Veal. 1999. Comparison of Cryptosporidium-specific and Giardia-specific monoclonal antibodies for monitoring water samples. Water Research 33, no. 7: 1611-1617. 
Fleischmann, N, K Staubmann, and G Langergraber. 2002. Management of sensible water uses with real-time measurements. Water science and technology : a journal of the International Association on Water Pollution Research 46, no. 3: 33-40.

Forster, S, H.M. Lappin-Scott, J.R. Snape, and J. Porter. 2003. Rains, drains and active strains: towards online assessment of wastewater bacterial communities. Journal of Microbiological Methods 55, no. 3: 859-864.

Gerhardt, Almut, and Stefanie Schmidt. 2002. The multispecies freshwater biomonitor a potential new tool for sediment biotests and biomonitoring. Journal of Soils and Sediments 2, no. 2: 67-70.

Gheewala, S. H., M. S. Babel, A. D. Gupta, and S Babel. 2003. Rapid Assessment techniques for chemicals in raw water sources. Jurnal of Water Supply: Research and Technology - AQUA 52.7: 521-528.

Hach. Chlorine, Free: method 8021 DPD method 1. Germany: Hach.

Hach. Chlorine, Total: method 8167 DPD method 1. Germany: Hach.

Hach. 2006. Model 2100AN Laboratory Turbidimeter Instrument Manual. 4th. Mississagua, ON: Hach Company.

Hall, John, A.D. Zaffiro, R.B. Marx, P.C. Kefauver, E. Krishnan, E. Radha, R.C. Haught, and Jonathan G Herrmann. 2007. On-line water quality parameters as indicators of distribution system contamination. Journal AWWA 99, no. 1: 66-77.

Hammes, F, F Goldschmidt, M Vital, Y Wang, and Thomas Egli. 2010. Measurement and interpretation of microbial adenosine tri-phosphate (ATP) in aquatic environments. Water Research 44, no. 13: 3915-3923.

Harris, Daniel C. 2003. Quantitative Chemical Analysis. 6th. New York, NY: W.H. Freeman and Company.

Hart, Barry T., Ian D. McKelvie, and Richard L. Benson. 1993. Real-time instrumentation for monitoring water quality: An Australian perspective. Trends in Analytical Chemistry 12, no. 10: 403-412.

Health Canada. 2006. Guidelines for Canadian Drinking Water - Technical Documents. Water Quality. www.hc-sc.gc.ca.

Health Canada. 2008. Guidelines for Canadian Drinking Water Quality Summary Table. Ottawa, ON. www.hc-sc.gc.ca.

Her, Namguk, Gary Amy, David Foss, Jaeweon Cho, Yeomin Yoon, and Paul Kosenka. 2002. Optimization of Method for Detecting and Characterizing NOM by HPLC-Size 
Exclusion Chromatography with UV and On-Line DOC Detection. Environmental Science \& Technology 36, no. 5: 1069-1076.

Hur, Jin, Soon-Jin Hwang, and Jae-Ki Shin. 2008. Using Synchronous Fluorescence Technique as a Water Quality Monitoring Tool for an Urban River. Water, Air, and Soil Pollution 191, no. 1-4: 231-243.

Janssens de Bisthoven, Luc, Almut Gerhardt, and Amadeu M V M Soares. 2004. Effects of acid mine drainage on larval Chironomus (Diptera, Chironomidae) measured with the Multispecies Freshwater Biomonitor. Environmental toxicology and chemistry / SETAC 23, no. 5: 1123-8.

Jenkins, M, R Feyer, J Trout, R Palmer, K Short, M Cosio, and C Horn. 2001. Hand-held Advanced Nucleic Acid Analyzer (HANNAA) for Waterborne Pathogen Detection. Alexandria, VA (99-HHE-4-ET).

Jian, S. 2006. Moleclar alternative to indicators and pathogen detection: Real-time PCR. Alexandria, VA ( ES-01-HHE-2a).

Katsoyiannis, I.A. 2006. Comparative evaluation of conventional and alternative methods for the removal of arsenic from contaminated groundwaters. Reviws and environmental health 21, no. 1: 25-41.

Kawamura, Kunio. 2003. In situ UV-VIS detection of the association of water-soluble anionic porphyrin and aromatic bases in aqueous solution at high tempeatures using a capillary flow hydrothermal reactor system. Analytical sciences : the international journal of the Japan Society for Analytical Chemistry 19, no. 8: 1199-202.

Kikkoman Corporation. Lumitester C-110 Operation Manual. Kikkoman Corporation.

Kollu, Kerim. 2008. Effects of Particles and Bioflocculation on Ultraviolet Disinfection. Engineering.

Kullman, S.W., D.E. Hilton, and K.G. Linden. 2009. Moving Towards and Innovative DNA Array Technology for Detection of Pharmaceuticals in Reclaimed Water. Alexandria, VA (703-684-2470).

Langergraber, G, J K Gupta, A Pressl, F Hofstaedter, W Lettl, A Weingartner, and N Fleischmann. 2004. On-line monitoring for control of a pilot-scale sequencing batch reactor using a submersible UV/VIS spectrometer. Water science and technology 50, no. 10: $73-80$.

Lee, J, and R Deininger. 2004. Detection of E. coli in beach water within 1 hour using immunomagnetic separation and ATP bioluminescence. Luminescence 19: 31-36. 
Lee, Jiyoung, and Rolf a Deininger. 2010. Real-Time Determination of the Efficacy of Residual Disinfection to Limit Wastewater Contamination in a Water Distribution System Using Filtration-Based Luminescence. Water Environment Research 82, no. 5: 475-478.

Lehmann, M, C Chan, A Lo, M Lung, K Tag, G Kunze, K Riedel, B Gruendig, and R Renneberg. 1999. Measurement of biodegradable substances using the salt-tolerant yeast Arxula adeninivorans for a microbial sensor immobilized with poly(carbamoyl)sulfonate (PCS). Part II: Application of the novel biosensor to real samples from coastal and island regions. Biosensors \& bioelectronics 14, no. 3: 295-302.

Leitão, João M M, and Joaquim C G Esteves Da Silva. 2010. Firefly luciferase inhibition. Journal of photochemistry and photobiology B: Biology 101, no. 1: 1-8.

LuminUltra Technologies. Preliminary chlorine and UV experiments to increase ATP reduction in disinfection processes pre-treatment before ATP analysis. Fredericton, NB.

LuminUltra Technologies. 2008. Product Fact Sheet - QGA Quench Gone Aqueous Formulation. Fredericton, NB: LuminUltra Technologies Inc.

LuminUltra Technologies. 2009. LumiSolve Material Safety Data Sheet. Annals of emergency medicine. Vol. 27. Fredericton: LuminUltra Technologies Inc.

LuminUltra Technologies. 2009. Product Fact Sheet - Kikkoman Lumitester C-110. Fredericton: LuminUltra Technologies Inc.

LuminUltra Technologies. 2010. Quotation. Test. Fredericton, NB: LuminUltra Technologies.

LuminUltra Technologies. 2010. Test Kit Instructions: Quench-Gone Aqueous Tet Kit Product \# QGA-25/QGA-100. Frederiction, NB: LuminUltra Technologies.

Meriluoto, J, K Karlsson, and L Spoof. 2004. throughput screening of ten microcystins and nodularins, cyanobacterial peptide hepatotoxins, by reversed-phase liquid chromatography-electrospray ionisation mass. Chromatographia 59: 291-298.

Metcalf \& Eddie. 2003. Wastewater Engineering: treatment and reuse. G Tchobanoglous, Franklin L Burton, and H David Stensel. 4th. Boston, MA: McGraw-Hill.

Mikol, Y.B., W.R. Richardson, W.H. can Der Schalie, and T.R. Shedd. 0207. An online real-time biomonitor for contaminated surveillance in water supplies. American Water Works Association Journal 99, no. 2: 107-114.

Mountfort, Douglas O, Patrick Holland, and Jan Sprosen. 2005. Method for detecting classes of microcystins by combination of protein phosphatase inhibition assay and ELISA: comparison with LC-MS. Toxicon 45, no. 2: 199-206. 
Nollet, L(ed.). 2007. Handbook of Water Analysis. 2nd. Boca Raton, FL: CRC Press.

Ontario Ministry of the Environment (MOE). 2003. Technical Support Document for Ontario Drinking Water Standards, Objectives and Guidelines. Toronto, ON.

Ontario Ministry of the Environment (MOE). 2008. Drinking Water Systems under O.Reg. 170/03. Ontario Drinking Water Legislation. www.ontario.ca.

Organisation for Economic Co-operation and Development (OECD), and World Health Organization (WHO). 2003. Assessing microbial safety of drinking water: improving approaches and methods. Paris, France: OECD Publishing.

Pang, Hei-Leung, Nga-Yan Kwok, Pak-Ho Chan, Chi-Hung Yeung, Waihung Lo, and Kwok-Yin Wong. 2007. High-Throughput Determination of Biochemical Oxygen Demand (BOD) by a Microplate-Based Biosensor. Environmental Science \& Technology 41, no. 11: 4038-4044.

Paul, D.H. 2003. Bioterrorism and Water Treatment: Part 1. Water Conditioning \& Purification October.

Poynton, Helen C., and Chris D. Vulpe. 2009. Ecotoxicogenomics: Emerging Technologies for Emerging Contaminants. Journal of the American Water Resources Association 45, no. 1: 83-96.

Rao, A.S. 2006. Introduction to Microbiology. 5th. New Delhi: Prentice-Hall of India.

Richardson, Susan D, and Thomas a Ternes. 2005. Water analysis: emerging contaminants and current issues. Analytical chemistry 77, no. 12: 3807-38.

Richardson, Susan D. 2009. Water analysis: emerging contaminants and current issues. Analytical chemistry 81, no. 12: 4645-77. doi:10.1021/ac9008012.

Rodriguez, Miguel, Charlene A Sanders, and Elias Greenbaum. 2002. Biosensors for rapid monitoring of primary-source drinking water using naturally occurring photosynthesis. Biosensors \& bioelectronics 17, no. 10: 843-9.

Rodriguez-Mozaz, Sara, Maria J Lopez De Alda, and Damià Barceló. 2007. Advantages and limitations of on-line solid phase extraction coupled to liquid chromatography-mass spectrometry technologies versus biosensors for monitoring of emerging contaminants in water. Journal of Chromatography A 1152, no. 1-2: 97-115.

Sakaguchi, Toshifumi, Yasunori Morioka, Masahiro Yamasaki, Junpei Iwanaga, Kazuhiko Beppu, Hideaki Maeda, Yasutaka Morita, and Eiichi Tamiya. 2007. Rapid and onsite BOD sensing system using luminous bacterial cells-immobilized chip. Biosensors \& bioelectronics 22 , no. $7:$ 1345-50. 
Schmittgen, T D. 2001. Real-time quantitative PCR. Methods 25: 383-385.

Schreppel, C.K., P.A. Tangorra, D.D. Eaton, and S.P. Donovan. 2002. On-line real time monitoring - Peace of mind?. In American Water WOrks Association - Water Technology Conference. American Water Works Association.

Schwarzenback, R.P., P.M. Gschwend, and D.M. Imboden. 2002. Environmental Organic Chemistry. 2nd. New Jersey: John Wiley and Sons.

Shimomura, Osamu. 2006. Bioluminescence: chemical principles and methods. Hackensack, NJ: World Scientific Publishing Co.

Sluyts, Hilde, François Van Hoof, Anja Cornet, and Jozef Paulussen. 1996. A dynamic new alarm system for use in biological early warning systems. Environmental Toxicology and Chemistry 15, no. 8: 1317-1323.

States, S., M Scheuring, J Kuchta, J Newberry, and L Casson. 2002. Microbial screening methods for ensuring security of public water supplies. In Water Works AssociationWater Quality Technology Conference.. American Water Works Association.

Straub, Timothy M, Brian P Dockendorff, Maria D Quiñonez-Díaz, Catherine O Valdez, Janani I Shutthanandan, Barbara J Tarasevich, Jay W Grate, and Cynthia J Bruckner-Lea. 2005. Automated methods for multiplexed pathogen detection. Journal of microbiological methods 62, no. 3: 303-16.

Szaecka, A, Y Xu, and P Tang. 2007. Dynamics of Firefly Luciferase Inhibition by General Anesthetcs: Gaussian and Anisotropic Network Analysis. Biophysical Journal 93, no. 6: 1895-1905.

Tanaka, H., T. Shinji, K. Sawada, Y. Monji, S. Seto, M. Yajima, and O. Yagi. 1997. Development and application of a bioluminescence ATP assay method for rapid detection of coliform bacteria. Water Research 31, no. 8: 1913-1918.

Thermo Fisher Scientific. 2007. Fisher Scientific (catalogue). Ottawa, ON: Termo Fisher Scientific. www.fishersci.ca.

Tschmelak, Jens, Guenther Proll, and Guenter Gauglitz. 2005. Optical biosensor for pharmaceuticals, antibiotics, hormones, endocrine disrupting chemicals and pesticides in water: Assay optimization process for estrone as example. Talanta 65, no. 2: 313-23.

U.S. Environmental Protecoitn Agency (US EPA). 2005. Technologies and Techniques for Early Warning Systems to Monitor and Evaluate Drinking Water Quality: A State-ofthe-Art Review. Washington, DC. 
United States Environmental Protection Agency. 2005. Technologies and Techniques for Early Warning Systems to Monitor and Evaluate Drinking Water Quality: A State-of-theArt Review. Washingon, DC. (EPA/600/R-05/156).

United States Environmental Protection Agency. 2006. Ultraviolet Disinfection Guidance Manal for the Final Long Term 2 Enhanced Surface Water Treatment Rule. Washington, DC.

Vaiopoulou, E, P Melidis, E Kampragou, and A Aivasidis. 2005. On-line load monitoring of wastewaters with a respirographic microbial sensor. Biosensors \& bioelectronics 21 , no. $2: 365-71$.

Vaitomaa, J, A Rantala, K Halinen, L Rouhiainen, P Tallberg, L Mokelke, and K Sivonen. 2003. Quantitative real-time PCR for determination of microcystin synthetase E copy numbers for Microcystis and Anabaena in lakes. Applied Environmental Microbiology 69, no. 12: 7289-7297.

van Der Schalie, W H, T R Shedd, M W Widder, and L M Brennan. 2004. Response characteristics of an aquatic biomonitor used for rapid toxicity detection. Journal of Applied Toxicology 24, no. 5: 387-94.

Water Environment Federation (WEF). 1996. Wastewater Disinfection. Alexandria, VA: Water Environment Federation.

Yuan, Moucun, and Wayne W Carmichael. 2004. Detection and analysis of the cyanobacterial peptide hepatotoxins microcystin and nodularin using SELDI-TOF mass spectrometry. Toxicon 44, no. 5: 561-70.

Zhou, W., K. Kageyama, F. Li, and A. Yuasa. 2007. Monitoring of Microbiological Water Quality by Real-Time PCR. Environmental Technology 28, no. 5: 545-553. 


\section{APPENDIX A}

\section{APPENDIX A: RAW DATA}

\section{Preliminary Phase: Quality Control and Assurance}

Table A 1: Testing the light leakage into the luminometer and from the cuvette.

\begin{tabular}{|r|lr|l|l|r|}
\hline $\begin{array}{l}\text { Replicate } \\
\text { No. }\end{array}$ & $\begin{array}{l}\text { Empty } \\
\text { Chamber }\end{array}$ & $\begin{array}{l}\text { Empty } \\
\text { Cuvette }\end{array}$ & $\begin{array}{l}\text { 2 Drops of } \\
\text { UltraCheque1 }\end{array}$ & $\begin{array}{l}\text { 2 drops of UltraCheque } \\
+2 \text { drops of Luminase }\end{array}$ \\
\hline 1 & 0 & 4 & 3 & 12,133 \\
\hline 2 & 1 & 3 & 3 & 13,242 \\
\hline 3 & 1 & 3 & 4 & 12,424 \\
\hline \hline Avg & 1 & 3 & 3 & 12,600 \\
\hline \multicolumn{1}{c|}{ std dev } & 1 & 1 & 1 & 575 \\
\hline
\end{tabular}

Table A 2: Background light readings of LuminUltra reagents

\begin{tabular}{|r|r|}
\hline Replicate No. & RLU \\
\hline \multicolumn{2}{|c|}{ XXL Luminase } \\
\hline 1 & 57 \\
\hline 2 & 49 \\
\hline Regular Luminase \\
\hline 1 & 7 \\
\hline 2 & 7 \\
\hline
\end{tabular}


Table A 3: Background light levels of deionized water and peptone water

\begin{tabular}{|c|c|c|c|c|c|c|c|c|}
\hline \multicolumn{2}{|c|}{ Ultracheque } & \multicolumn{7}{|l|}{8,807} \\
\hline $\begin{array}{c}\text { Sample } \\
\text { No. }\end{array}$ & Description & $\begin{array}{l}\text { Vol. of } \\
\text { Sample } \\
\text { Filtered } \\
\text { (mL) }\end{array}$ & $\begin{array}{c}\text { Amount } \\
\text { of Light } \\
\text { (RLU) }\end{array}$ & $\begin{array}{c}\text { Avg } \\
\text { Amount } \\
\text { of Light } \\
\text { (RLU) }\end{array}$ & Std Dev & $\begin{array}{l}\text { Amount } \\
\text { of ATP } \\
\text { (pg } \\
\text { ATP/mL) }\end{array}$ & $\begin{array}{c}\text { Avg } \\
\text { Amout } \\
\text { of ATP } \\
\text { (pg } \\
\text { ATP/mL) }\end{array}$ & Std Dev \\
\hline 1 & \multirow{4}{*}{$\begin{array}{c}\text { Autoclaved } \\
\text { D.I. water }\end{array}$} & 10.0 & 20 & 19 & 2 & 2.3 & \multirow[b]{4}{*}{2.1} & 0.2 \\
\hline & & 10.0 & 20 & & & 2.3 & & \\
\hline \multirow[t]{2}{*}{2} & & 10.0 & 17 & & & 1.9 & & \\
\hline & & 10.0 & 17 & & & 1.9 & & \\
\hline 3 & \multirow{4}{*}{$\begin{array}{c}\text { Autoclaved } \\
0.1 \text { percent } \\
\text { Peptone }\end{array}$} & 10.0 & 30 & 39 & 6 & 3.4 & \multirow[b]{4}{*}{4.4} & 0.7 \\
\hline & & 10.0 & 41 & & & 4.7 & & \\
\hline & & 10.0 & 41 & & & 4.7 & & \\
\hline & & 10.0 & 43 & & & 4.9 & & \\
\hline
\end{tabular}

Table A 4: Membrane filtration results and calculations from repeatability study

\begin{tabular}{|r|c|r|r|r|r|r|r|}
\hline $\begin{array}{c}\text { Sample } \\
\text { Number }\end{array}$ & $\begin{array}{c}\text { Dilution of } \\
\text { Initial } \\
\text { Experimental } \\
\text { Solution }\end{array}$ & $\begin{array}{c}\text { Dilution } \\
\text { Factor } \\
\text { of } \\
\text { Sample } \\
\text { for MF }\end{array}$ & $\begin{array}{c}\text { Vol. of } \\
\text { Diluted } \\
\text { Sample } \\
\text { Filtered } \\
\text { [mL] }\end{array}$ & $\begin{array}{c}\text { \# CFU } \\
\text { on } \\
\text { Agar }\end{array}$ & CFU /mL & $\begin{array}{c}\text { AVG CFU } \\
\text { /mL }\end{array}$ & Std Dev \\
\hline 1 & No Dilution & 100,000 & 25 & 35 & $1.4 \mathrm{E}+05$ & $1.4 \mathrm{E}+05$ & $4.0 \mathrm{E}+03$ \\
\hline & & 100,000 & 25 & 34 & $1.4 \mathrm{E}+05$ & & \\
\hline & & 100,000 & 25 & 36 & $1.4 \mathrm{E}+05$ & & \\
\hline 2 & 0.01 & 10,000 & 25 & 4 & $2 \mathrm{E}+03$ & $3 \mathrm{E}+03$ & $1 \mathrm{E}+03$ \\
\hline & & 10,000 & 25 & 9 & $4 \mathrm{E}+03$ & & \\
\hline 3 & 0.001 & 10,000 & 25 & 10 & $4.0 \mathrm{E}+03$ & & \\
\hline & & 100 & 25 & 28 & $1.1 \mathrm{E}+02$ & $1.2 \mathrm{E}+02$ & $1.1 \mathrm{E}+01$ \\
\hline & & 100 & 25 & 32 & $1.3 \mathrm{E}+02$ & & \\
\hline
\end{tabular}


Table A 5: ATP assay results and calculations from repeatability study

UltraCheque 1

\begin{tabular}{|c|c|c|c|c|c|c|}
\hline \multirow{2}{*}{ UltraCheque 1} & & \multicolumn{5}{|c|}{ ATP Assay - 0.7 um Filter } \\
\hline & $\begin{array}{l}\text { Replicate } \\
\text { No. }\end{array}$ & $\begin{array}{l}\text { Vol. of } \\
\text { Sample } \\
\text { Filtered } \\
(\mathrm{mL})\end{array}$ & $\begin{array}{l}\text { Amount } \\
\text { of Light } \\
\text { Measured } \\
\text { (RLU) }\end{array}$ & $\begin{array}{l}\text { Amount of } \\
\text { ATP } \\
(\mathrm{pg} / \mathrm{mL})\end{array}$ & $\begin{array}{l}\text { AVG ATP } \\
\text { (pg/mL) }\end{array}$ & $\begin{array}{l}\text { Std. } \\
\text { Dev. }\end{array}$ \\
\hline $\begin{array}{l}\text { Conc of } \\
\text { Bacteria } \\
\text { (CFU/mL) }\end{array}$ & 1 & 50.0 & 3,250 & 42.9 & 36.2 & 5.9 \\
\hline $1.4 \mathrm{E}+05$ & 2 & 50.0 & 2,714 & 35.9 & & \\
\hline $1.4 \mathrm{E}+05$ & 3 & 50.0 & 2,876 & 38.0 & & \\
\hline $1.4 \mathrm{E}+05$ & 4 & 50.0 & 2,829 & 37.4 & & \\
\hline $1.4 \mathrm{E}+05$ & 5 & 50.0 & 2,024 & 26.7 & & \\
\hline $1.4 \mathrm{E}+05$ & 1 & 50.0 & 72 & 1.2 & 1.3 & 0.3 \\
\hline $3 E+03$ & 2 & 50.0 & 91 & 1.5 & & \\
\hline $3 E+03$ & 3 & 50.0 & 61 & 1.0 & & \\
\hline $3 E+03$ & 4 & 50.0 & 106 & 1.71 & & \\
\hline $3 E+03$ & 5 & 50.0 & 76 & 1.2 & & \\
\hline $3 E+03$ & 1 & 50.0 & 32 & 0.42 & 0.33 & 0.07 \\
\hline $1.1 \mathrm{E}+02$ & 2 & 50.0 & 22 & 0.29 & & \\
\hline $1.1 \mathrm{E}+02$ & 3 & 50.0 & 28 & 0.37 & & \\
\hline $1.1 E+02$ & 4 & 50.0 & 23 & 0.30 & & \\
\hline $1.1 \mathrm{E}+02$ & 5 & 50.0 & 19 & 0.25 & & \\
\hline $1.1 \mathrm{E}+02$ & & & & & & \\
\hline
\end{tabular}


Table A 6: Turbidity measurements

\begin{tabular}{|c|c|c|c|}
\hline Sample & $\begin{array}{l}\text { Turbidity } \\
\text { (NTU) }\end{array}$ & $\begin{array}{l}\text { Avg Turbidity } \\
\text { (NTU) }\end{array}$ & Std Dev \\
\hline \multirow[b]{3}{*}{ River Water } & 2.63 & 2.56 & 0.0987 \\
\hline & 2.61 & & \\
\hline & 2.45 & & \\
\hline \multirow{3}{*}{$\begin{array}{c}\text { River Water + } \\
0.25 \text { mL Clay } \\
\text { Sol'n }\end{array}$} & 6.18 & 5.95 & 0.1992 \\
\hline & 5.83 & & \\
\hline & 5.84 & & \\
\hline \multirow{3}{*}{$\begin{array}{c}\text { River Water + } \\
0.5 \text { mL Clay } \\
\text { Sol'n }\end{array}$} & 9.79 & 9.82 & 0.2616 \\
\hline & 10.10 & & \\
\hline & 9.58 & & \\
\hline \multirow{3}{*}{$\begin{array}{c}\text { River Water + } \\
1.0 \mathrm{~mL} \text { Clay } \\
\text { Sol'n }\end{array}$} & 17.10 & 16.63 & 0.4509 \\
\hline & 16.60 & & \\
\hline & 16.20 & & \\
\hline \multirow{3}{*}{$\begin{array}{c}\text { River Water + } \\
1.5 \text { mL Clay } \\
\text { Sol'n }\end{array}$} & 23.40 & 23.00 & 0.3606 \\
\hline & 22.90 & & \\
\hline & 22.70 & & \\
\hline \multirow{3}{*}{$\begin{array}{l}\text { River Water + } \\
2.0 \mathrm{~mL} \text { Clay } \\
\text { Sol'n }\end{array}$} & 32.70 & 32.43 & 0.5508 \\
\hline & 31.80 & & \\
\hline & 32.80 & & \\
\hline
\end{tabular}

Table A 7: ATP assay results and calculations from turbidity

\begin{tabular}{|c|c|c|c|c|c|c|}
\hline \multicolumn{5}{|l|}{ UltraCheck1 } & 336,522 & 22,843 \\
\hline Sample & $\begin{array}{l}\text { Avg } \\
\text { Turbidity } \\
\text { (NTU) }\end{array}$ & Std Dev & $\begin{array}{l}\text { Vol. of } \\
\text { Sample } \\
\text { Filtered } \\
(\mathrm{mL}) \\
\end{array}$ & (RLU) & $\mathrm{RLU} / \mathrm{ml}$ & $\begin{array}{l}\text { Amount } \\
\text { of ATP } \\
\text { (pg/mL) }\end{array}$ \\
\hline \multirow[b]{2}{*}{ River Water } & 2.56 & 0.10 & 15.0 & 236,936 & 15,796 & 469.38 \\
\hline & 2.56 & 0.10 & 15.0 & 261,849 & 17,457 & 518.74 \\
\hline \multirow{2}{*}{$\begin{array}{c}\text { River Water }+0.25 \\
\text { mL Clay Sol'n }\end{array}$} & 5.95 & 0.20 & 15.0 & 183,307 & 12,220 & 363.14 \\
\hline & 5.95 & 0.20 & 15.0 & 196,378 & 13,092 & 389.03 \\
\hline \multirow{2}{*}{$\begin{array}{c}\text { River Water + } 0.5 \\
\text { mL Clay Sol'n }\end{array}$} & 9.82 & 0.26 & 15.0 & 167,507 & 11,167 & 331.84 \\
\hline & 9.82 & 0.26 & 15.0 & 209,662 & 13,977 & 415.35 \\
\hline \multirow{2}{*}{$\begin{array}{c}\text { River Water }+1.0 \\
\text { mL Clay Sol'n }\end{array}$} & 16.63 & 0.45 & 15.0 & 221,538 & 14,769 & 438.88 \\
\hline & 16.63 & 0.45 & 15.0 & 184,555 & 12,304 & 365.61 \\
\hline \multirow{2}{*}{$\begin{array}{l}\text { River Water + } 1.5 \\
\text { mL Clay Sol'n }\end{array}$} & 23.00 & 0.36 & 15.0 & 198,320 & 13,221 & 392.88 \\
\hline & 23.00 & 0.36 & 15.0 & 174,161 & 11,611 & 345.02 \\
\hline
\end{tabular}


River Water +2.0 $\mathrm{mL}$ Clay Sol'n

32.43

32.43

0.55

\begin{tabular}{l|l}
15.0 & 204,808 \\
\hline
\end{tabular}

13,654

405.73

$15.0 \quad 212,586$

14,172

421.14

Table A 8: ANOVA test performed on the turbidity data

\begin{tabular}{lrrrrr}
\hline \multicolumn{1}{c}{ Source } & DF & $\begin{array}{c}\text { Sum of } \\
\text { squares }\end{array}$ & $\begin{array}{c}\text { Mean } \\
\text { squares }\end{array}$ & F & $\operatorname{Pr}>$ F \\
\hline Model & 5 & 22263.067 & 4452.613 & 2.972 & 0.109 \\
Error & 6 & 8988.116 & 1498.019 & & \\
$\begin{array}{l}\text { Corrected } \\
\text { Total }\end{array}$ & 11 & 31251.183 & & & \\
\hline \multicolumn{4}{l}{ Computed against model $Y=$ Mean $(Y)$} \\
\end{tabular}

Computed against model $Y=$ Mean $(Y)$ 
ส

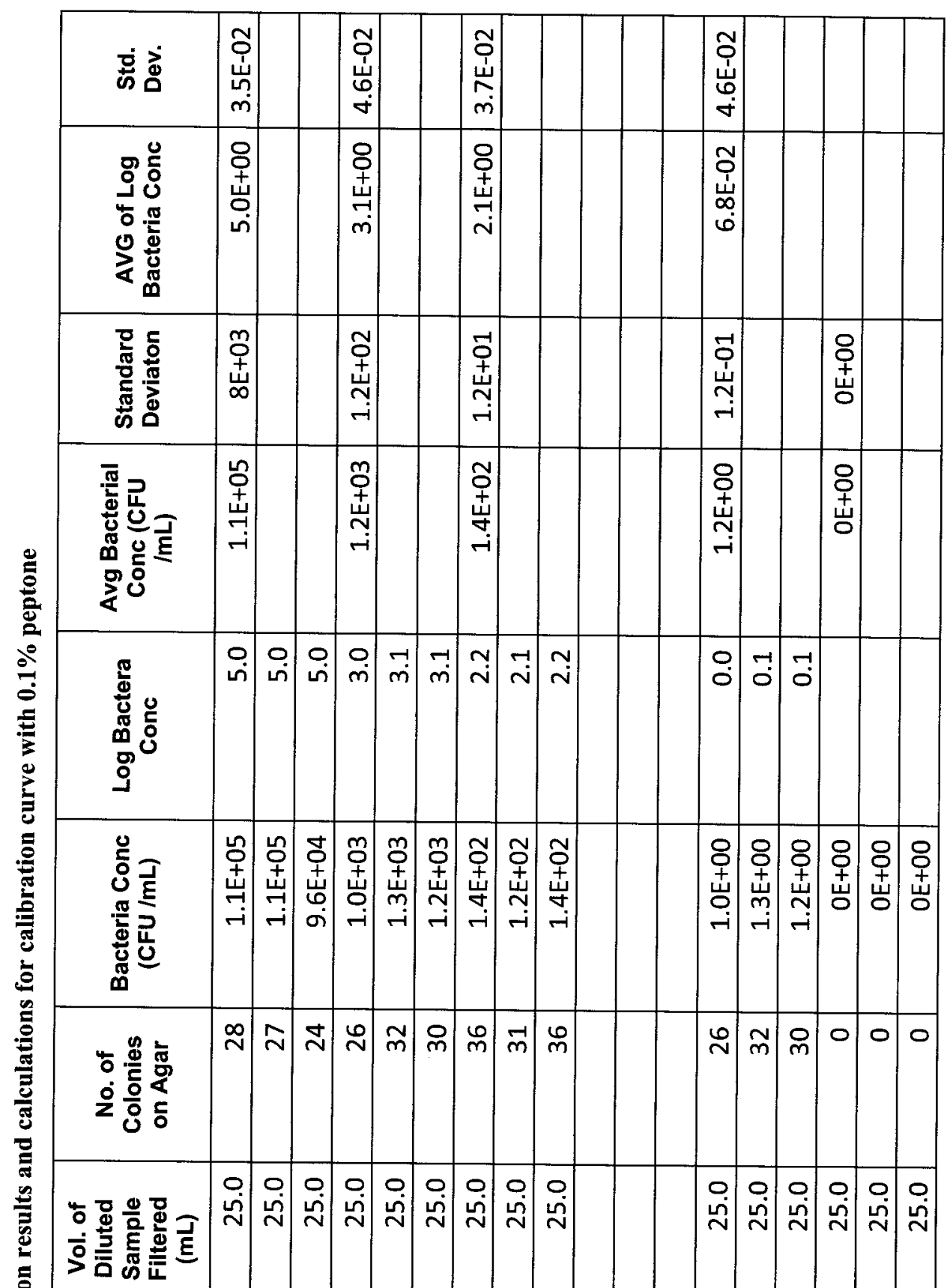

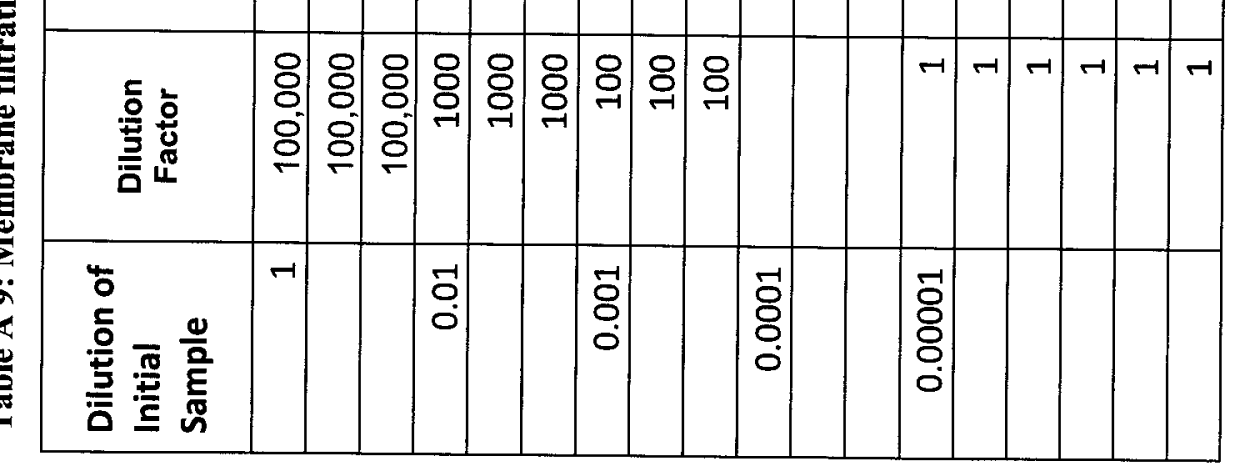




\begin{tabular}{|c|c|c|c|c|c|c|c|c|c|c|c|c|c|c|}
\hline & 离离 & $\left|\begin{array}{l}\infty \\
0 \\
0 \\
0 \\
0\end{array}\right|$ & & $\left|\begin{array}{l}n \\
0 \\
0 \\
0 \\
0\end{array}\right|$ & & $\mid$\begin{tabular}{l|}
$\overrightarrow{0}$ \\
0 \\
0
\end{tabular} & & $\begin{array}{l}9 \\
\\
0\end{array}$ & & $\mid \begin{array}{l}\stackrel{0}{m} \\
\vdots \\
0\end{array}$ & & $\mid \begin{array}{l}0 \\
0 \\
0\end{array}$ & & \\
\hline & 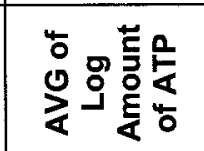 & 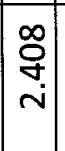 & & $\left|\begin{array}{c}\hat{o} \\
0 \\
0 \\
\tilde{m}\end{array}\right|$ & & \begin{tabular}{|l|}
$\vec{\sigma}$ \\
i
\end{tabular} & & $\mid$ & & ฟี & & \begin{tabular}{|l|} 
\\
\end{tabular} & & \\
\hline & 站高 & \begin{tabular}{|l|}
$\infty$ \\
$\dot{i n}$
\end{tabular} & & $\mid$\begin{tabular}{|c|}
$\mid \begin{array}{l}\mid \\
\vdots \\
\vdots\end{array}$ \\
\end{tabular} & & $\mid \begin{array}{l}0 \\
\dot{S}\end{array}$ & & \begin{tabular}{|l|} 
\\
$\infty$ \\
$\infty$
\end{tabular} & & $\begin{array}{c}\tilde{T} \\
\tilde{y}\end{array}$ & & \begin{tabular}{|l|l|} 
\\
0 \\
\end{tabular} & & \\
\hline & 旅套 & 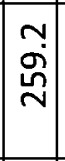 & & 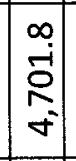 & & $\mid \begin{array}{l}\mathbb{N} \\
\mathbb{\mathcal { J }}\end{array}$ & & \begin{tabular}{|l}
$\mid$ \\
$\stackrel{n}{m}$ \\
\end{tabular} & & 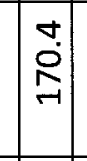 & & I & & \\
\hline & 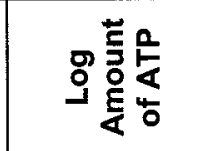 & : & \્ْડ & $\left|\begin{array}{l}0 \\
0 \\
0 \\
\dot{m}\end{array}\right|$ & 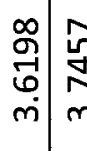 & ⿵⺆ & 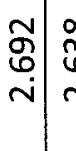 & 竎 & 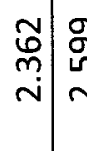 & 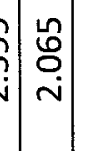 & & $\stackrel{m}{0}$ & & $\stackrel{7}{0}$ \\
\hline & 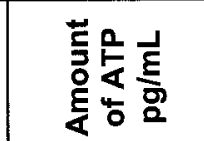 & 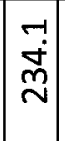 & 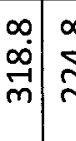 & $\begin{array}{l}\hat{\rho} \\
\hat{o} \\
g\end{array}$ & 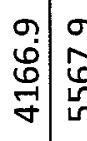 & 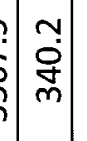 & बें & \begin{tabular}{|l|} 
\\
0 \\
$m$
\end{tabular} & 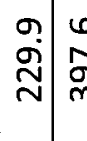 & $\mid$\begin{tabular}{l}
7 \\
$\vdots$ \\
0 \\
\hdashline
\end{tabular} & & $\nexists$ & & I \\
\hline & $\vec{\not}$ & 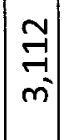 & 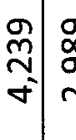 & $\mid$ & 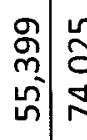 & 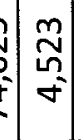 & 象: & $\vec{\exists}$ & 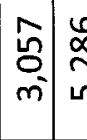 & 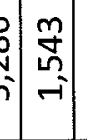 & & $\stackrel{\infty}{\sim}$ & & $\stackrel{\infty}{\sim}$ \\
\hline 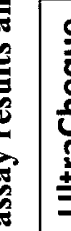 & 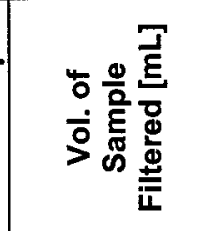 & 品 & $\stackrel{9}{\circ}$ & $\begin{array}{l}0 \\
0 \\
\end{array}$ & $\begin{array}{l}0 \\
\stackrel{-}{0}\end{array}$ & \begin{tabular}{ll}
0 \\
\hdashline \\
\hdashline
\end{tabular} & 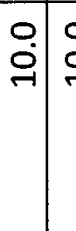 & : & 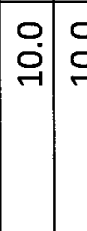 & 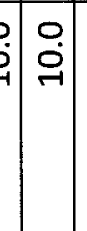 & $\begin{array}{ll}0 \\
\stackrel{9}{0}\end{array}$ & \begin{tabular}{l}
0 \\
0 \\
\hdashline
\end{tabular} & & $\stackrel{\circ}{\circ}$ \\
\hline & 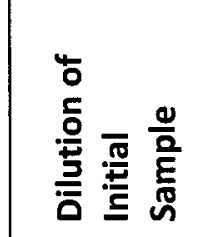 & -7 & & $\mid \begin{array}{l}\mid \\
0 \\
0\end{array}$ & & $\left|\begin{array}{l}\vec{b} \\
0 \\
0\end{array}\right|$ & & 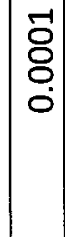 & & $\left|\begin{array}{l}\mid \overrightarrow{0} \\
0 \\
0 \\
0\end{array}\right|$ & & & & \\
\hline
\end{tabular}


ส

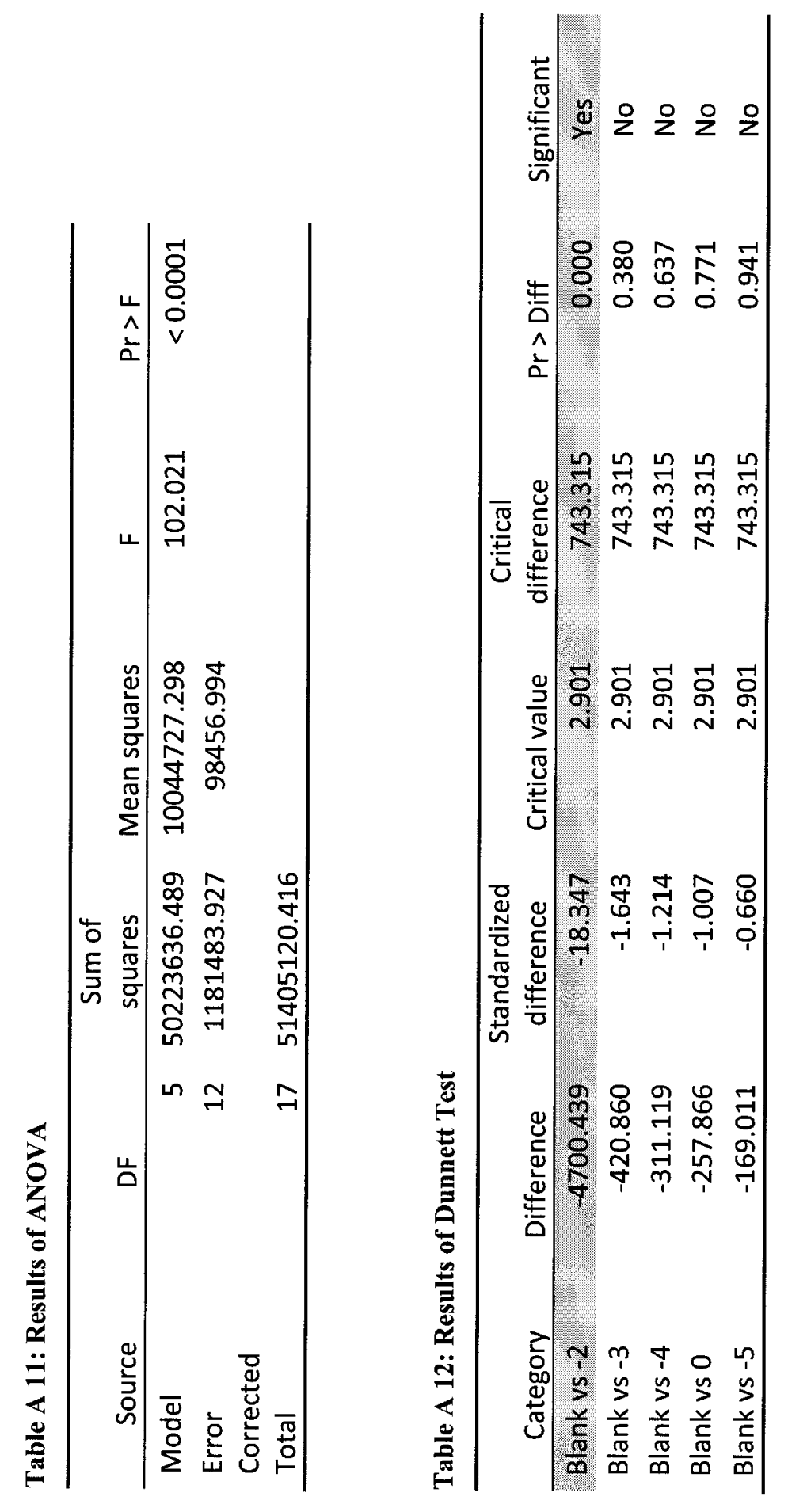




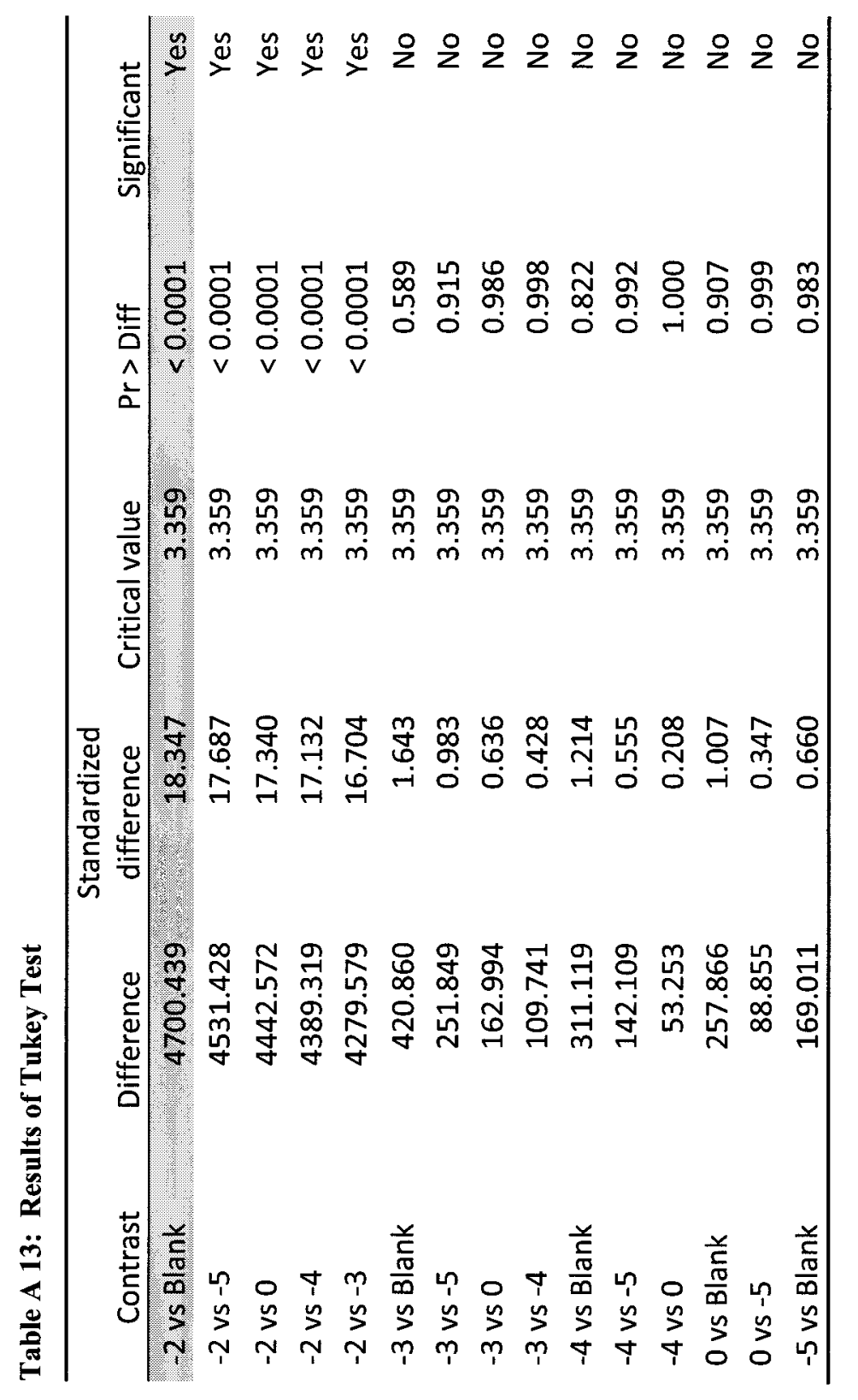


ส

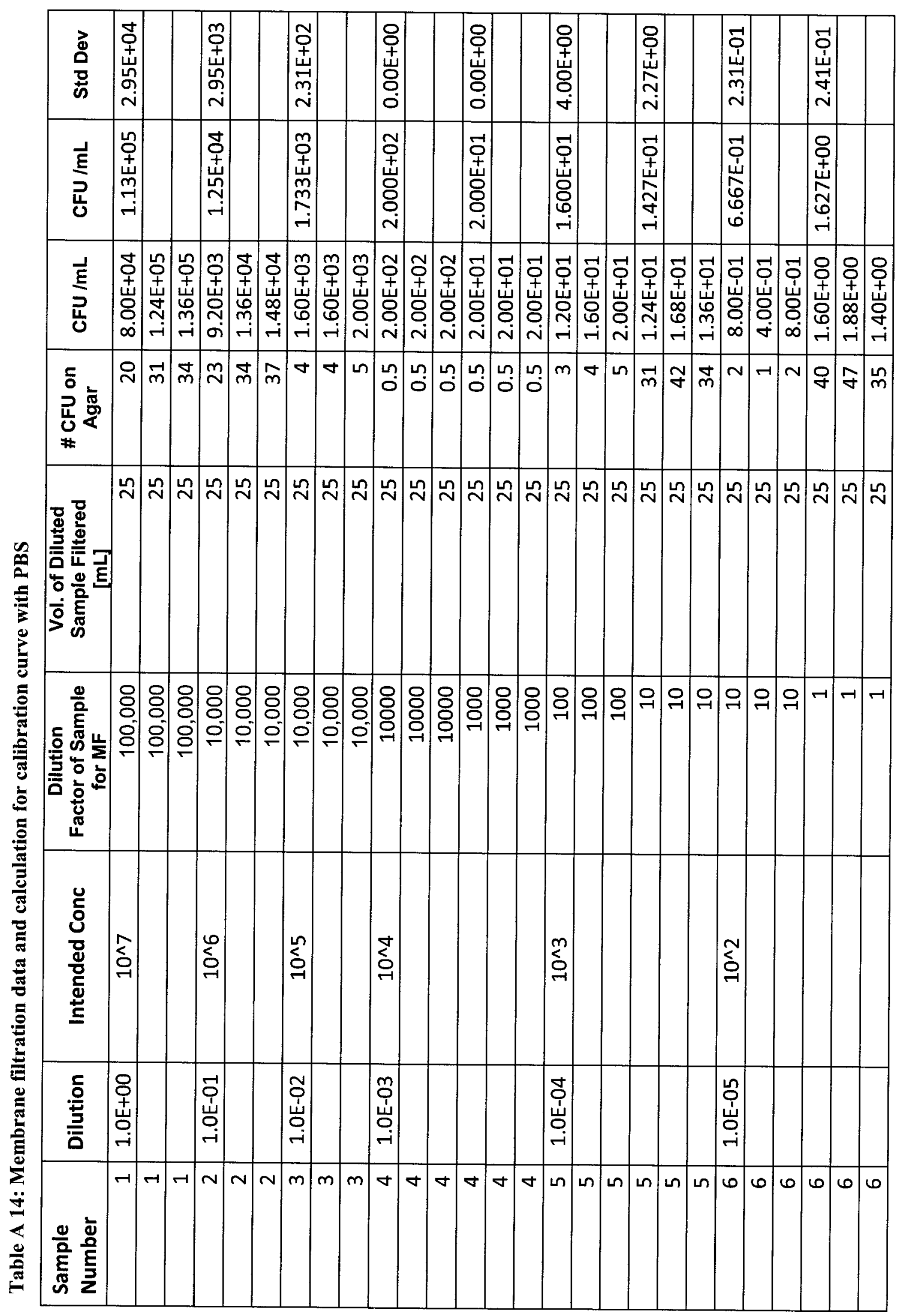


สั
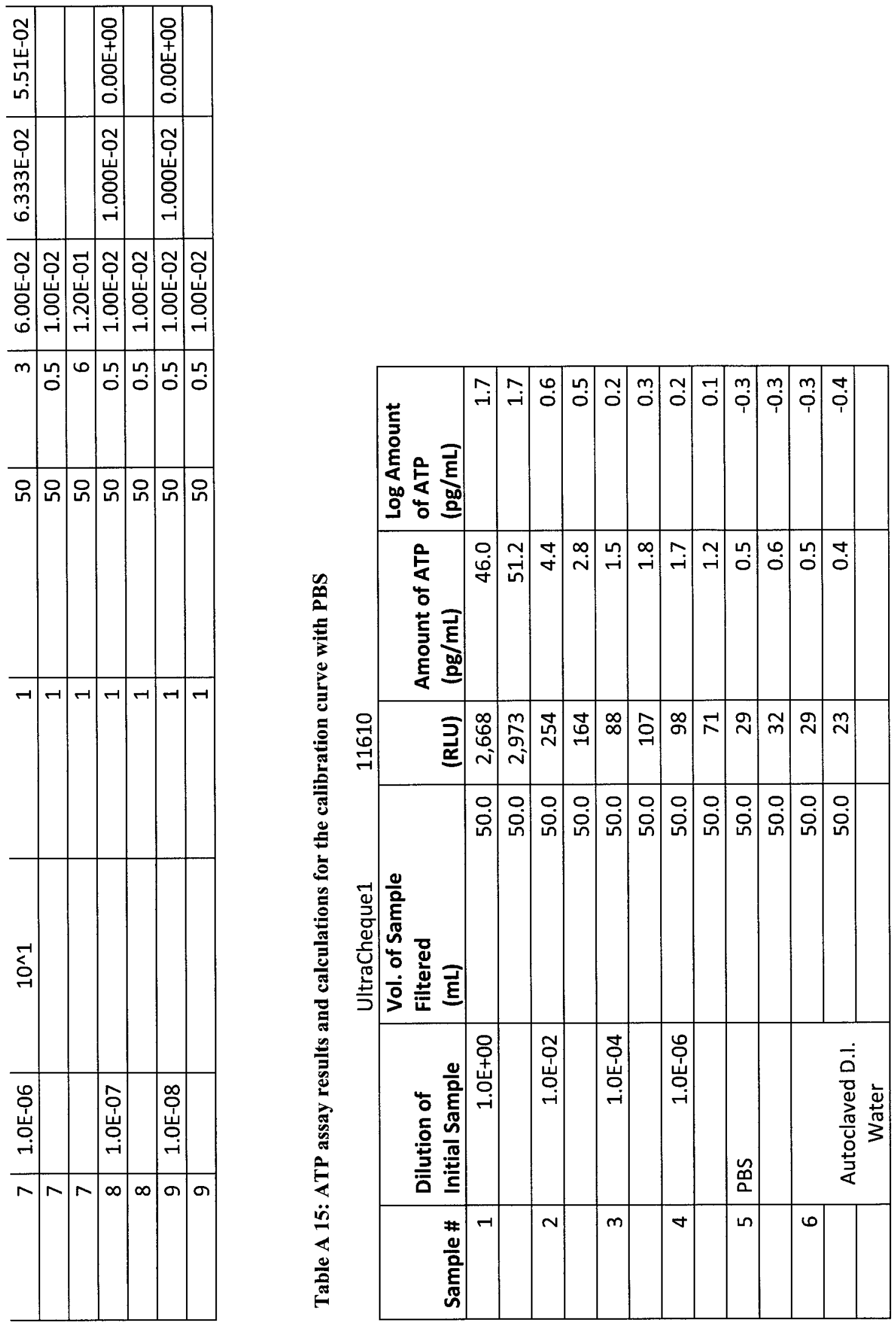


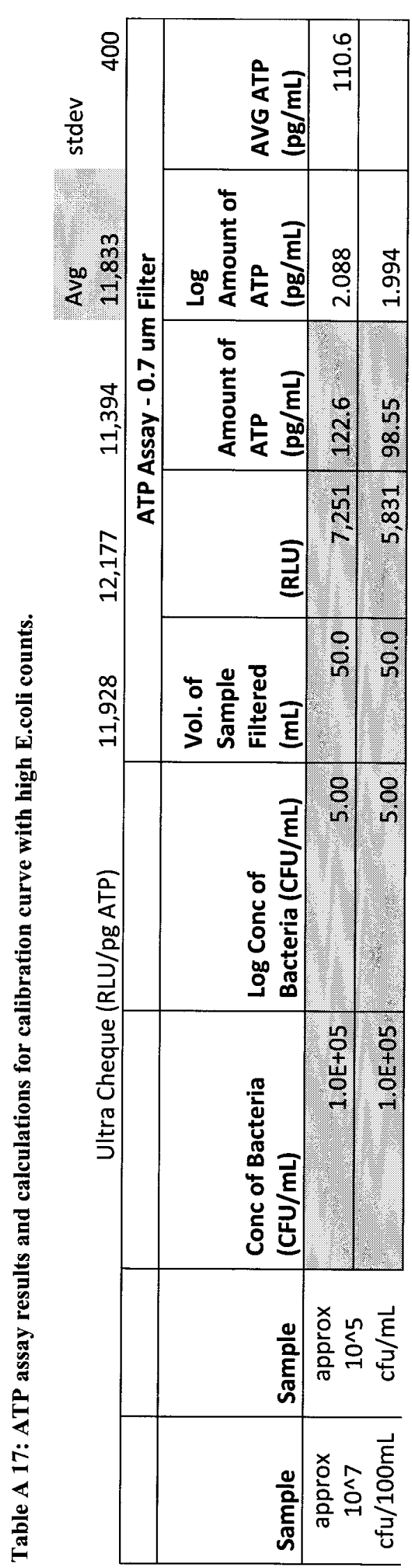




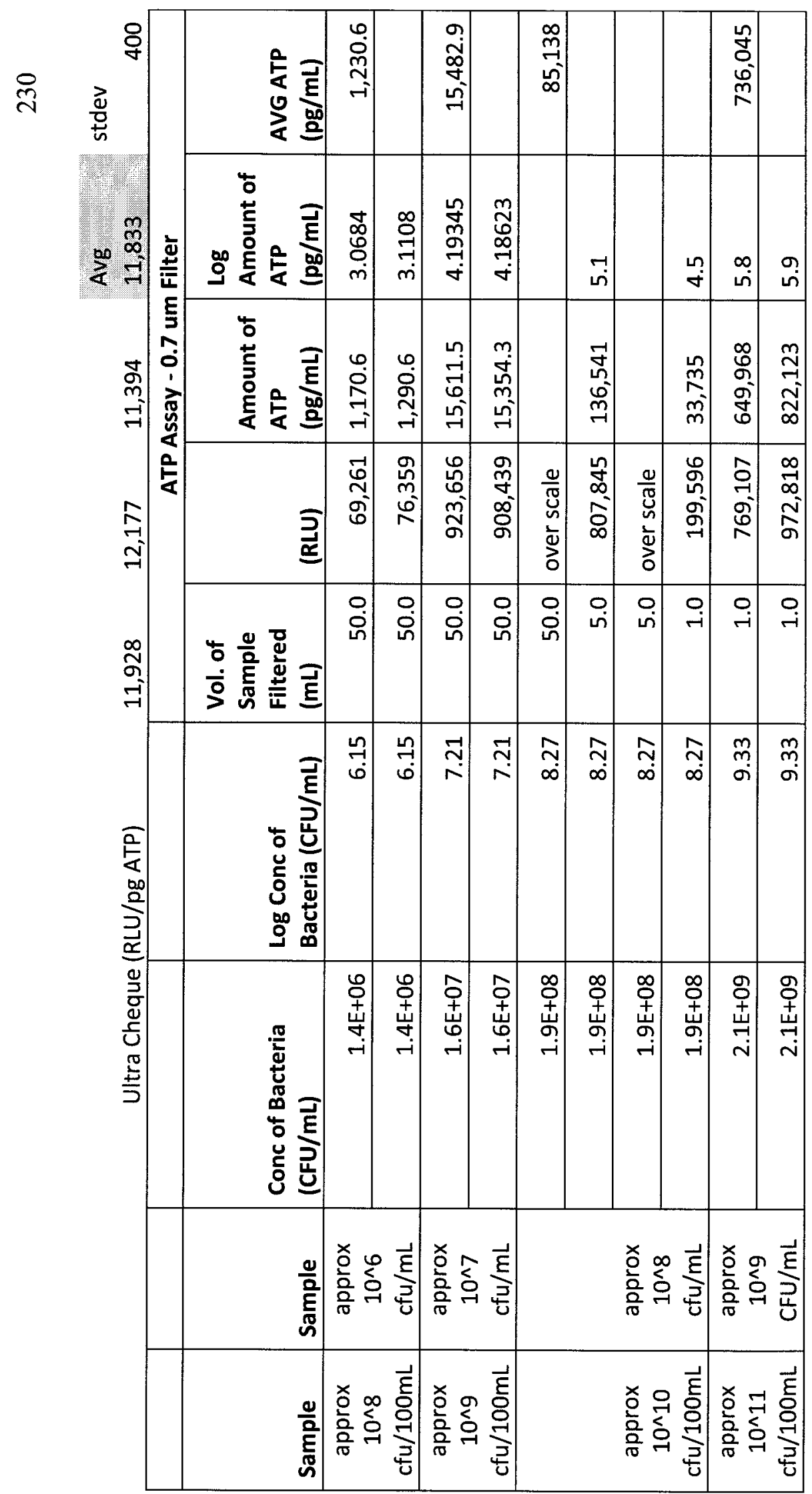


Phase I: Optimization of the ATP Assay

Table A 18: Membrane filtration data and calculations for the effect of varying the pore size of the filter

\begin{tabular}{|r|c|r|r|r|r|r|l|}
\hline $\begin{array}{r}\text { Sample } \\
\text { Number }\end{array}$ & $\begin{array}{c}\text { Dilution of } \\
\text { Initial } \\
\text { Solution }\end{array}$ & $\begin{array}{c}\text { Dilution } \\
\text { Factor }\end{array}$ & $\begin{array}{c}\text { Vol. of } \\
\text { Sample } \\
\text { Filtered } \\
\text { [mL] }\end{array}$ & $\begin{array}{c}\text { \# } \\
\text { CFU } \\
\text { on } \\
\text { Agar }\end{array}$ & CFU /mL & $\begin{array}{c}\text { AVG CFU } \\
\text { /mL }\end{array}$ & Std Dev \\
\hline 1 & No Dilution & 100,000 & 25 & 27 & $1.08 \mathrm{E}+05$ & $1.17 \mathrm{E}+05$ & $3.11 \mathrm{E}+04$ \\
\hline 1 & & 100,000 & 25 & 38 & $1.52 \mathrm{E}+05$ & & \\
\hline 1 & & 100,000 & 25 & 23 & $9.20 \mathrm{E}+04$ & & \\
\hline 2 & $1.0 \mathrm{E}-02$ & 1,000 & 25 & 28 & $1.12 \mathrm{E}+03$ & $1.00 \mathrm{E}+03$ & $1.06 \mathrm{E}+02$ \\
\hline 2 & & 1,000 & 25 & 24 & $9.60 \mathrm{E}+02$ & & \\
\hline 2 & & 1,000 & 25 & 23 & $9.20 \mathrm{E}+02$ & & \\
\hline 3 & $1.0 \mathrm{E}-04$ & 10 & 25 & 9 & $3.60 \mathrm{E}+00$ & $8.67 \mathrm{E}+00$ & $4.82 \mathrm{E}+00$ \\
\hline 3 & & 10 & 25 & 33 & $1.32 \mathrm{E}+01$ & & \\
\hline 3 & & 10 & 25 & 23 & $9.20 \mathrm{E}+00$ & & \\
\hline 4 & PBS & 1 & 25 & 0.5 & $2.00 \mathrm{E}-02$ & $2.00 \mathrm{E}-02$ & $0.00 \mathrm{E}+00$ \\
\hline 4 & & 1 & 25 & 0.5 & $2.00 \mathrm{E}-02$ & & \\
\hline 4 & & 1 & 25 & 0.5 & $2.00 \mathrm{E}-02$ & & \\
\hline 5 & DI Water & 1 & 25 & 0.5 & $2.00 \mathrm{E}-02$ & $2.00 \mathrm{E}-02$ & $0.00 \mathrm{E}+00$ \\
\hline 5 & & 1 & 25 & 0.5 & $2.00 \mathrm{E}-02$ & & \\
\hline 5 & & 1 & 25 & 0.5 & $2.00 \mathrm{E}-02$ & & \\
\hline
\end{tabular}


Table A 19: ATP assay results and calculations for the effect of varying the size of the filter

\begin{tabular}{|c|c|c|c|c|}
\hline Ultra Cheque (RLU/pg ATP) & & & & 11,610 \\
\hline & \multicolumn{4}{|c|}{$0.7 \mu \mathrm{m}$ Filter } \\
\hline Sample \# & $\begin{array}{l}\text { Vol. of Sample } \\
\text { Filtered }(\mathrm{mL})\end{array}$ & (RLU) & $\begin{array}{l}\text { Amount of ATP } \\
(\mathrm{pg} / \mathrm{mL})\end{array}$ & $\begin{array}{l}\text { AVG ATP } \\
(\mathrm{pg} / \mathrm{mL})\end{array}$ \\
\hline \multirow{2}{*}{$\begin{array}{c}\text { Sample } 1 \text { - approx } 10^{\wedge} 5 \\
\mathrm{cfu} / \mathrm{mL}\end{array}$} & 50 & 2,668 & 45.96 & 48.59 \\
\hline & 50 & 2,973 & 51.21 & \\
\hline \multirow{2}{*}{$\begin{array}{c}\text { Sample } 2 \text { approx } 10^{\wedge} 3 \\
\mathrm{cfu} / \mathrm{mL}\end{array}$} & 50 & 254 & 4.38 & 3.60 \\
\hline & 50 & 164 & 2.83 & \\
\hline \multirow{2}{*}{$\begin{array}{c}\text { Sample } 3 \text { - approx } 10^{\wedge} 1 \\
\text { cfu/mL }\end{array}$} & 50 & 88 & 1.52 & 1.68 \\
\hline & 50 & 107 & 1.84 & \\
\hline \multirow[b]{2}{*}{ Sample 4 - PBS } & 50 & 29 & 0.50 & 0.53 \\
\hline & 50 & 32 & 0.55 & \\
\hline \multirow{4}{*}{ Sample 5 - D.I. Water } & 50 & 29 & 0.50 & 0.45 \\
\hline & 50 & 23 & 0.40 & \\
\hline & & & & \\
\hline & \multicolumn{4}{|c|}{$0.2 \mu \mathrm{m}$ Filter } \\
\hline Sample \# & $\begin{array}{l}\text { Vol. of Sample } \\
\text { Filtered (mL) }\end{array}$ & (RLU) & $\begin{array}{l}\text { Amount of ATP } \\
(\mathrm{pg} / \mathrm{mL})\end{array}$ & \\
\hline $\begin{array}{c}\text { Sample } 1 \text { - approx } 10^{\wedge} 5 \\
\mathrm{cfu} / \mathrm{mL}\end{array}$ & 10 & 292 & 25.15 & \\
\hline $\begin{array}{l}\text { Sample } 2 \text { approx } 10^{\wedge} 3 \\
\text { cfu/mL }\end{array}$ & 10 & 98 & 8.44 & \\
\hline $\begin{array}{c}\text { Sample } 3 \text { - approx } 10^{\wedge} 1 \\
\mathrm{cfu} / \mathrm{mL}\end{array}$ & 10 & 71 & 6.12 & \\
\hline Sample 4 - PBS & 10 & 26 & 2.24 & \\
\hline & 10 & 21 & 1.81 & \\
\hline Sample 5 - D.I. Water & 10 & 20 & 1.72 & \\
\hline
\end{tabular}


Table A 20: Membran filtration data and calculations for calibration curve with low bacteria counts with Luminase and XXL Luminase

\begin{tabular}{|c|c|c|c|c|c|c|c|c|}
\hline $\begin{array}{l}\text { Intended } \\
\text { Conc }\end{array}$ & $\begin{array}{c}\text { Dilution } \\
\text { Factor of } \\
\text { Sample } \\
\text { for MF }\end{array}$ & $\begin{array}{c}\text { Vol. of } \\
\text { Diluted } \\
\text { Sample } \\
\text { Filtered } \\
\text { [mL] }\end{array}$ & $\begin{array}{c}\text { \# CFU on } \\
\text { Agar }\end{array}$ & $\begin{array}{l}\text { CFU } \\
/ \mathrm{mL}\end{array}$ & $\begin{array}{c}\log \\
\text { CFU/mL }\end{array}$ & $\begin{array}{l}\text { AVG } \\
\text { CFU } \\
/ \mathrm{mL}\end{array}$ & $\begin{array}{l}\text { Std } \\
\text { Dev }\end{array}$ & $\begin{array}{l}\text { log } \\
\text { Std } \\
\text { Dev }\end{array}$ \\
\hline $10^{\prime} 1 \mathrm{CFU} / \mathrm{mL}$ & 1.E+01 & 25.0 & 30 & 1.2E+01 & 1.1E+00 & 1.0E+01 & $2 E+00$ & $1 \mathrm{E}-01$ \\
\hline & 1.E+01 & 25.0 & 27 & $1.1 \mathrm{E}+01$ & $1.0 \mathrm{E}+00$ & & & \\
\hline & 1.E+01 & 25.0 & 19 & $7.6 \mathrm{E}+00$ & $8.8 \mathrm{E}-\mathrm{O} I$ & 2 & & 2 \\
\hline \multirow[t]{3}{*}{$10^{\wedge} 1 \mathrm{CFU} / \mathrm{mL}$} & 1.E+02 & 25.0 & 0 & $\mathrm{OE}+00$ & & $3 E+00$ & $5 E+00$ & \\
\hline & 1.E+02 & 25.0 & 0 & $\mathrm{OE}+00$ & & & & \\
\hline & 1.E+02 & 25.0 & 2 & $8 \mathrm{E}+00$ & & & & \\
\hline $10 \wedge 3 \mathrm{CFU} / \mathrm{mL}$ & 1.E+03 & 25.0 & 25 & 1.0E+03 & 3.0E+00 & $1.1 E+03$ & $1 E+02$ & $5 E-02$ \\
\hline & $1 E+03$ & 25,0 & 25 & 1.0E +03 & 3. $0 E+00$ & & & \\
\hline & 4 E+03 & 25.0 & 30 & 1.2 E*0 & $3.1 \mathrm{E}+00$ & 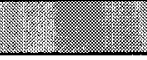 & & \\
\hline \multirow[t]{3}{*}{$10^{\wedge} 3 \mathrm{CFU} / \mathrm{mL}$} & $1 . E+04$ & 25.0 & 0 & $\mathrm{OE}+00$ & & $7 E+02$ & $6 \mathrm{E}+02$ & \\
\hline & $1 . E+04$ & 25.0 & 3 & $1 \mathrm{E}+03$ & & & & \\
\hline & 1.E+04 & 25.0 & 2 & $8 \mathrm{E}+02$ & & & & \\
\hline \multirow[t]{3}{*}{$1015 \mathrm{CFU} / \mathrm{mL}$} & 1.E+05 & 25.0 & 22 & $8.8 E+04$ & $4.9 E+00$ & $9.5 E+04$ & $8 E+03$ & $4 E-02$ \\
\hline & 1.E+05 & 25.0 & 23 & $9.2 E+04$ & $5.0 E+00$ & & & \\
\hline & $1 . E+05$ & 25.0 & 26 & 1.OE +05 & $5.0 E+00$ & & & \\
\hline \multirow[t]{3}{*}{$10^{\wedge} 5 \mathrm{CFU} / \mathrm{mL}$} & 1. $E+06$ & 25.0 & 5 & $2 E+05$ & & $1 \mathrm{E}+05$ & $8 \mathrm{E}+04$ & \\
\hline & 1.E+06 & 25.0 & 4 & $2 E+05$ & & & & \\
\hline & 1. $E+06$ & 25.0 & 1 & $4 \mathrm{E}+04$ & & & & \\
\hline \multirow[t]{3}{*}{$10^{\wedge} 8 \mathrm{CFU} / \mathrm{mL}$} & 1. $E+07$ & 25.0 & Overgrowth & & & & & \\
\hline & 1.E+07 & 25.0 & Overgrowth & & & & & \\
\hline & 1.E+07 & 25.0 & Overgrowth & & & & & \\
\hline \multirow[t]{3}{*}{$1018 \mathrm{CFU} / \mathrm{mL}$} & 1. $E+08$ & 25.0 & 27 & $1.1 E+08$ & & $1.7 E+08$ & $5 E+07$ & \\
\hline & 1.E+08 & 25.0 & 48 & $1.9 E+0.8$ & & & 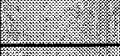 & \\
\hline & $1 . E+08$ & 25.0 & 49 & 2. OE+08 & & & $2=0$ & \\
\hline \multirow[t]{3}{*}{$10^{\wedge} 8 \mathrm{CFU} / \mathrm{mL}$} & 1. $E+09$ & 25.0 & 4 & $2 \mathrm{E}+08$ & & $2 \mathrm{E}+08$ & $6 \mathrm{E}+07$ & \\
\hline & 1.E+09 & 25.0 & 6 & $2 \mathrm{E}+08$ & & & & \\
\hline & 1.E+09 & 25.0 & 7 & $3 \mathrm{E}+08$ & & & & \\
\hline
\end{tabular}


Table A 21: ATP assay data and calculation for calibration curve with low bacteria counts and XXL Luminase

\begin{tabular}{|c|c|c|c|c|}
\hline Sample & $\begin{array}{l}\text { Vol. of Sample } \\
\text { Filtered }(\mathrm{mL})\end{array}$ & $\begin{array}{l}\text { Amount of } \\
\text { Light (RLU) }\end{array}$ & $\begin{array}{l}\text { Amount of ATP } \\
(\mathrm{pg} / \mathrm{mL})\end{array}$ & $\begin{array}{l}\text { Log Amount of } \\
\text { ATP }(\mathrm{pg} / \mathrm{mL})\end{array}$ \\
\hline \multirow{2}{*}{$\begin{array}{c}\text { approx } 10^{\wedge}-2 \\
\text { CFU } / \mathrm{mL}\end{array}$} & 1000 & 32,911 & 0.74 & -0.13 \\
\hline & 1000 & 15,345 & 0.35 & -0.46 \\
\hline \multirow{2}{*}{$\begin{array}{c}\text { approx } 10^{\wedge}-1 \\
\mathrm{CFU} / \mathrm{mL}\end{array}$} & 900 & 12,713 & 0.32 & -0.50 \\
\hline & 900 & 10,537 & 0.26 & -0.58 \\
\hline \multirow{2}{*}{$\begin{array}{c}\text { approx } 10^{\wedge} 0 \\
\mathrm{CFU} / \mathrm{mL}\end{array}$} & 900 & 12,682 & 0.32 & -0.50 \\
\hline & 900 & 3,683 & 0.09 & -1.03 \\
\hline \multirow{4}{*}{$\begin{array}{c}\text { approx } 10^{\wedge} 1 \\
\text { CFU/mL }\end{array}$} & 950 & 2,726 & 0.06 & -1.19 \\
\hline & 925 & 18,349 & 0.45 & -0.35 \\
\hline & 1000 & 5,561 & 0.13 & -0.90 \\
\hline & 990 & 15,911 & 0.36 & -0.44 \\
\hline \multirow{3}{*}{$\begin{array}{c}\text { approx } 10^{\wedge} 2 \\
\text { CFU/mL }\end{array}$} & 800 & 5,150 & 0.15 & -0.84 \\
\hline & 800 & 5,517 & 0.16 & -0.81 \\
\hline & 900 & 10,752 & 0.27 & -0.57 \\
\hline \multirow{2}{*}{$\begin{array}{c}\text { approx } 10^{\wedge} 3 \\
\mathrm{CFU} / \mathrm{mL}\end{array}$} & 500 & 13,809 & 0.62 & -0.21 \\
\hline & 500 & 17,958 & 0.81 & -0.09 \\
\hline \multirow{2}{*}{$\begin{array}{c}\text { approx } 10^{\wedge 5} 5 \\
\mathrm{CFU} / \mathrm{mL}\end{array}$} & 50 & 307,873 & 138.87 & 2.14 \\
\hline & 50 & 322,876 & 145.64 & 2.16 \\
\hline
\end{tabular}

Table A 22: ATP assay data and calculations for calibration curve with low bacteria counts and Luminase

Ultra Cheque (RLU/pg ATP)

\begin{tabular}{|c|c|c|c|c|}
\hline Sample & $\begin{array}{l}\text { Vol. of Sample } \\
\text { Filtered (mL) }\end{array}$ & $\begin{array}{l}\text { Amount of } \\
\text { Light (RLU) }\end{array}$ & $\begin{array}{l}\text { Amount of ATP } \\
(\mathrm{pg} / \mathrm{mL})\end{array}$ & $\begin{array}{l}\text { Log Amount of } \\
\text { ATP }(\mathrm{pg} / \mathrm{mL})\end{array}$ \\
\hline \multirow{3}{*}{$\begin{array}{c}\text { approx } 10^{\wedge} 1 \\
\text { CFU/mL }\end{array}$} & 950 & 396 & 0.45 & -0.34 \\
\hline & 1000 & 428 & 0.47 & -0.33 \\
\hline & 950 & 139 & 0.16 & -0.80 \\
\hline \multirow{2}{*}{$\begin{array}{c}\text { approx } 10^{\wedge} 2 \\
\mathrm{CFU} / \mathrm{mL}\end{array}$} & 800 & 129 & 0.18 & -0.76 \\
\hline & 800 & 144 & 0.20 & -0.71 \\
\hline \multirow{2}{*}{$\begin{array}{c}\text { approx } 10^{\wedge} 3 \\
\text { CFU/mL }\end{array}$} & 500 & 378 & 0.82 & -0.08 \\
\hline & 500 & 518 & 1.13 & 0.05 \\
\hline \multirow{2}{*}{$\begin{array}{l}\text { approx } 10^{\wedge 5} \\
\text { CFU/mL }\end{array}$} & 50 & 7,644 & 166.45 & 2.22 \\
\hline & 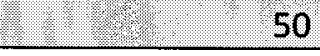 & 7,884 & 171.67 & 2.23 \\
\hline approx $10^{\wedge} 6$ & 1 & 1,704 & 1855.20 & 3.27 \\
\hline
\end{tabular}


Table A 23: Effect of changing the amount of lysing solution

\begin{tabular}{|c|c|c|c|}
\hline Amount of Ultra Lyse (mL) & $\begin{array}{l}\text { Measured Light } \\
\text { (RLU) }\end{array}$ & $\begin{array}{l}\text { Measured Light after } 5 \text { minutes } \\
\text { (RLU) }\end{array}$ & pH \\
\hline 1 & 1529 & 1122 & 11 \\
\hline 2 & 866 & 690 & 11.3 \\
\hline 3 & 429 & 511 & 11.5 \\
\hline
\end{tabular}

Table A 24: Membrane filtration data and calculations for calibration curve with XXL Luminase and low bacteria counts.

\begin{tabular}{|c|c|c|c|c|c|c|c|}
\hline Intended Conc & $\begin{array}{l}\text { Dilution } \\
\text { Factor of } \\
\text { Sample } \\
\text { for MF }\end{array}$ & $\begin{array}{c}\text { Vol. of } \\
\text { Diluted } \\
\text { Sample } \\
\text { Filtered } \\
\text { [mL] }\end{array}$ & $\begin{array}{c}\# \\
\text { CFU } \\
\text { on } \\
\text { Agar }\end{array}$ & $\begin{array}{l}\text { Bacteria Conc } \\
(\text { CFU } / \mathrm{mL})\end{array}$ & $\begin{array}{c}\text { Log Bacteria } \\
\text { Conc } \\
\text { (CFU/mL) }\end{array}$ & $\begin{array}{c}\text { Avg Bacteria } \\
\text { Conc } \\
\text { (CFU } / \mathrm{mL} \text { ) }\end{array}$ & Std Dev \\
\hline $10{ }^{3}$ CF U/mL & 1. $E+03$ & 25.0 & 43 & $1.7 E+03$ & 3.24 & $1.7 E+03$ & $2 E+02$ \\
\hline 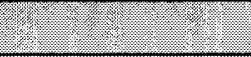 & 1.E+03 & 25.0 & 37 & 1.5E+03 & 3.17 & & \\
\hline 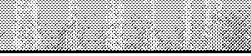 & $1 E+03$ & 25.0 & 45 & $1.8 \mathrm{E}+03$ & 3.26 & 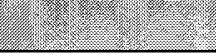 & \\
\hline $10^{\wedge} 3 \mathrm{CFU} / \mathrm{mL}$ & 1.E+04 & 25.0 & 3 & $1 \mathrm{E}+03$ & 3.1 & $2 \mathrm{E}+03$ & $1 E+03$ \\
\hline & 1. $E+04$ & 25.0 & 6 & $2 \mathrm{E}+03$ & 3.4 & & \\
\hline & 1. $E+04$ & 25.0 & 9 & $4 \mathrm{E}+03$ & 3.6 & & \\
\hline $10 \times 2 \mathrm{CFU} / \mathrm{mL}$ & 1.E+02 & 25.0 & 46 & $1.8 E+02$ & 2.26 & $1.8 \mathrm{E}+02$ & $2 \mathrm{E}+01$ \\
\hline & 1.E+02 & 25.0 & 48 & 19E+02 & 2.28 & & 3 \\
\hline & 1. $E+02$ & 25.0 & 39. & $1.6 \mathrm{E}+02$ & 2.19 & 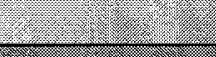 & $x^{2}$ \\
\hline 101190 & 1.E+01 & 25.0 & 47 & $1.98+04$ & 127 & $1.8 E+01$ & $2 E+00$ \\
\hline & 1.E+01 & 25.0 & 48 & 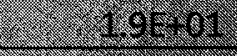 & 1.27 & I & \\
\hline 2 & 1.E+01 & 25.0 & 8 & 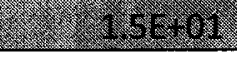 & 3.18 & 10 & $2 \sqrt{3}$ \\
\hline $10^{\wedge} 0 \mathrm{CFU} / \mathrm{mL}$ & $1 . E+02$ & 25.0 & 2 & $8.00 E+00$ & 0.903 & $1.07 E+01$ & $4.62 E+00$ \\
\hline & 1.E+02 & 25.0 & 4 & $1.60 \mathrm{E}+01$ & 1.204 & & \\
\hline & 1.E+02 & 25.0 & 2 & $8.00 \mathrm{E}+00$ & 0.903 & & \\
\hline 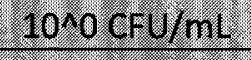 & 1. $E+\infty 0$ & 25.0 & 43. & 1.7E+00 & 0.236 & $1.6 \mathrm{E}+00$ & $2 E-01$ \\
\hline & 1. $E+00$ & 25.0 & 36 & 1. $4 E+00$ & 0.158 & & $8 E-01$ \\
\hline 2 & $1 . E+00$ & 25.0 & 43 & $1.7 E+00$ & 0.236 & & $9 E-01$ \\
\hline $\begin{array}{c}10^{\wedge} 1 \mathrm{CFU} / 100 \\
\mathrm{~mL}\end{array}$ & 1. $E+00$ & 100.0 & 15 & $1.50 \mathrm{E}-01$ & -0.824 & $1.50 \mathrm{E}-01$ & $2 \mathrm{E}-02$ \\
\hline & 1. $E+00$ & 100.0 & 17 & $1.70 \mathrm{E}-01$ & -0.770 & & \\
\hline & $1 . E+00$ & 100.0 & 13 & $1.30 \mathrm{E}-01$ & -0.886 & & \\
\hline $\begin{array}{c}10^{\wedge} 0 \mathrm{CFU} / 100 \\
\mathrm{~mL}\end{array}$ & 1. $E+00$ & 100.0 & 2 & $2.00 \mathrm{E}-02$ & -1.699 & 1.67E-02 & $1.53 \mathrm{E}-02$ \\
\hline
\end{tabular}




\begin{tabular}{|r|r|r|r|r|r|r|l|} 
& $1 . \mathrm{E}+00$ & 100.0 & 0 & $0.00 \mathrm{E}+00$ & & & \\
\hline & $1 . \mathrm{E}+00$ & 100.0 & 3 & $3.00 \mathrm{E}-02$ & -1.523 & & \\
\hline
\end{tabular}

Table A 25: ATP assay data and calculations for calibration curve with XXL Lumnase and low bacteria conts

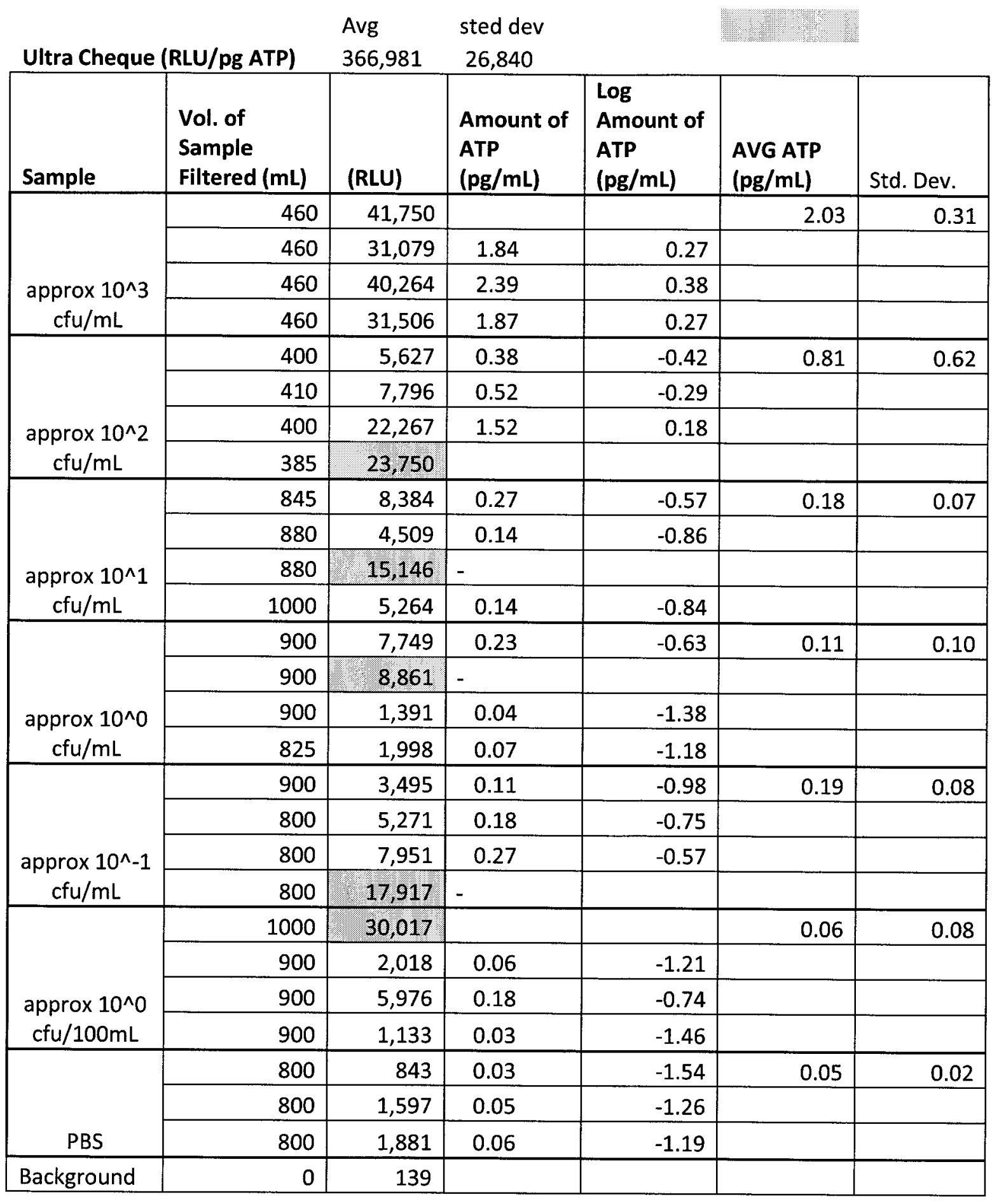


Table A 26: ANOVA test for calibration curve with XXL Luminase and low bacteria counts

\begin{tabular}{lcrrrrr}
\hline \multicolumn{1}{c}{$\begin{array}{l}\text { Source of } \\
\text { Variation }\end{array}$} & SS & $d f$ & MS & $F$ & P-value & F crit \\
\hline $\begin{array}{l}\text { Between } \\
\text { Groups } \\
\text { Within }\end{array}$ & 9.462192 & 6 & 1.577032 & 21.74933 & 06 & 2.847726 \\
Groups & 1.015132 & 14 & 0.072509 & & & \\
Total & 10.47732 & 20 & & & & \\
\hline
\end{tabular}




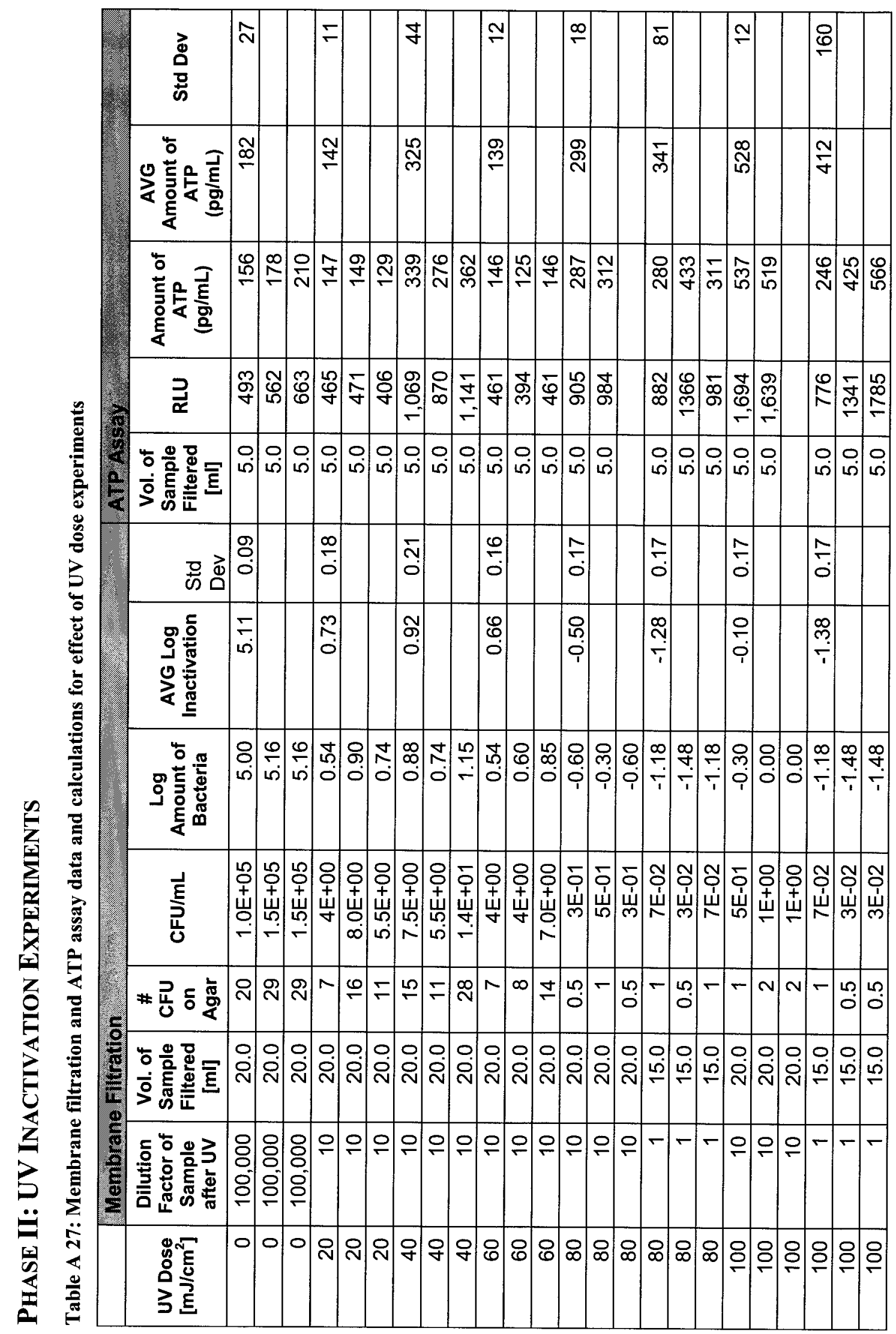




\begin{tabular}{|c|c|c|c|c|c|c|c|c|c|c|c|c|c|c|c|}
\hline & क्षे す & న్ & & & $\stackrel{2}{2}$ & & & $\mathscr{\infty}$ & & & 0 & & & & 요 \\
\hline & 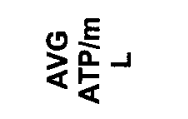 & 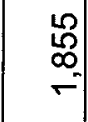 & & & م్ & & & $\begin{array}{l}R \\
N \\
=\end{array}$ & & & 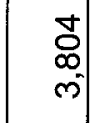 & & & & \\
\hline & 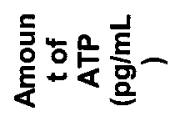 & 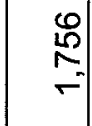 & $\begin{array}{l}\mathscr{8} \\
\stackrel{\infty}{\leftarrow}\end{array}$ & $\stackrel{\infty}{\stackrel{m}{\sigma}}$ & $\stackrel{?}{\stackrel{2}{2}}$ & $\underset{\stackrel{N}{\sim}}{-}$ & $\stackrel{9}{\stackrel{2}{-}}$ & م્م- & 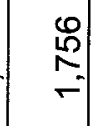 & 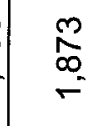 & $\begin{array}{l}\infty \\
\stackrel{0}{\circ} \\
m\end{array}$ & $\begin{array}{l}8 \\
8 \\
m\end{array}$ & & 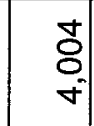 & $\frac{8}{\oplus}$ \\
\hline & 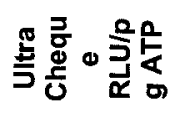 & & $\frac{9}{5}$ & $\frac{8}{2}$ & $\stackrel{0}{\stackrel{m}{-}}$ & $\stackrel{9}{\mathrm{~m}}$ & $\stackrel{0}{\mathrm{~m}}$ & $\begin{array}{l}\frac{8}{2} \\
i\end{array}$ & $\stackrel{\text { ㅇ }}{\mathrm{N}}$ & $\frac{0}{i}$ & $\begin{array}{l}m \\
0 \\
0 \\
0\end{array}$ & 离 & & 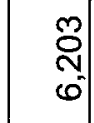 & $\begin{array}{l}\stackrel{8}{N} \\
0 \\
0\end{array}$ \\
\hline$\left|\begin{array}{l}\overrightarrow{3} \\
0 \\
0 \\
0\end{array}\right|$ & $\overrightarrow{\vec{\alpha}}$ & $\begin{array}{l}\overline{0} \\
\mathbb{N} \\
0\end{array}$ & $\begin{array}{l}ষ \\
\varnothing \\
\varnothing \\
6\end{array}$ & 음 & $\begin{array}{l}N \\
\stackrel{\infty}{N} \\
\sim\end{array}$ & $\begin{array}{l}1 \\
0 \\
0 \\
5\end{array}$ & 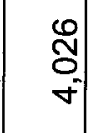 & 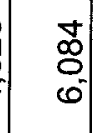 & 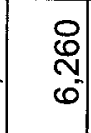 & 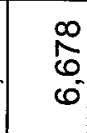 & $g^{g} 0$ & $\begin{array}{l}F^{N} \\
F^{2}\end{array}$ & 舫 & 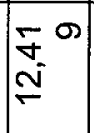 & $\begin{array}{l}\bar{\infty} \\
\sigma^{\circ}\end{array}$ \\
\hline $\begin{array}{l} \\
0 \\
\frac{1}{4} \\
\end{array}$ & 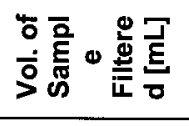 & 임 & $\begin{array}{l}0 \\
10\end{array}$ & $\begin{array}{l}0 \\
10\end{array}$ & 임 & 잉 & 잉 & $\begin{array}{l}0 \\
100\end{array}$ & $\begin{array}{l}0 \\
\\
0\end{array}$ & 웅 & $\begin{array}{l}0 \\
0^{\circ}\end{array}$ & \begin{tabular}{|l|}
0 \\
0 \\
0
\end{tabular} & $\begin{array}{l}0 \\
1\end{array}$ & 인 & مأ \\
\hline & 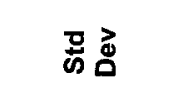 & $\begin{array}{l}0 \\
0 \\
0\end{array}$ & & & $\stackrel{m}{0}$ & & & $\begin{array}{l} \pm \\
\vdots \\
\vdots\end{array}$ & & & $\frac{N}{0}$ & & & & $\begin{array}{l}\mathscr{0} \\
\stackrel{0}{0}\end{array}$ \\
\hline & 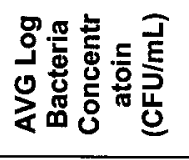 & 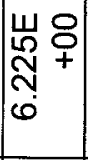 & & & 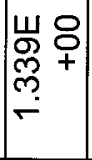 & & & 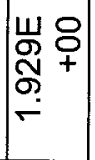 & & & $\mid$\begin{tabular}{|l}
$\omega$ \\
$\varrho$
\end{tabular} & & & & W \\
\hline & gơ & 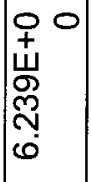 & $\mid \begin{array}{l}0 \\
+ \\
\dot{u} \\
0 \\
0 \\
0 \\
0\end{array}$ & \begin{tabular}{|ll}
0 & 0 \\
+ \\
$w$ \\
0 \\
$\hat{y}$ \\
0
\end{tabular} & 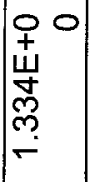 & 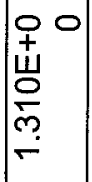 & 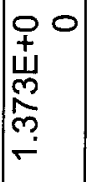 & 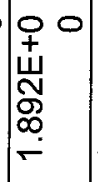 & 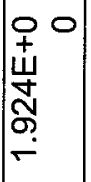 & 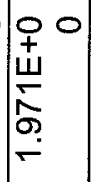 & 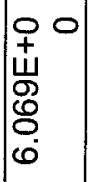 & 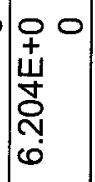 & 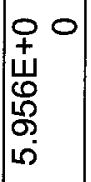 & & 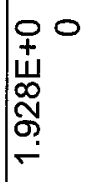 \\
\hline & 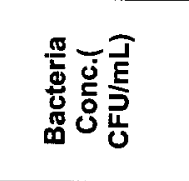 & $\left|\begin{array}{ll}0 & 0 \\
+ \\
w \\
N \\
\\
r\end{array}\right|$ & $\mid \begin{array}{l}0 \\
+ \\
\dot{W} \\
0 \\
\dot{q} \\
-\end{array}$ & $\mid \begin{array}{ll}0 & 0 \\
+ \\
\dot{w} \\
心 \\
\infty \\
-\end{array}$ & 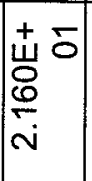 & 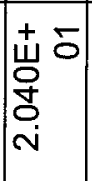 & 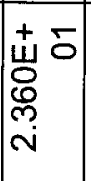 & 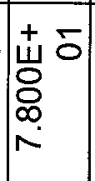 & 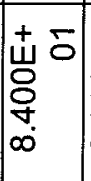 & 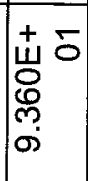 & 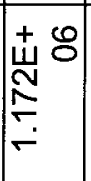 & 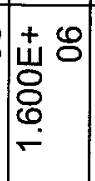 & 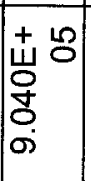 & & $\begin{array}{l}+ \\
\text { 岁 } \\
\infty \\
\infty \\
\infty\end{array}$ \\
\hline c & 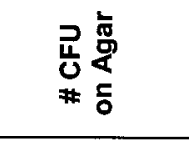 & 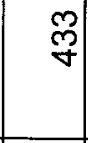 & 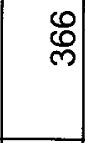 & $\mathscr{\&}$ & $\dot{\circ}$ & 5 & is & $\stackrel{10}{\circ}$ & 은 & స్ & న్సి & \& & $\stackrel{\mathbb{N}}{N}$ & & $\stackrel{N}{\frac{N}{N}}$ \\
\hline 尊 & 市离 & $\begin{array}{c}0 \\
\stackrel{0}{N}\end{array}$ & $\begin{array}{c}\circ \\
\stackrel{0}{\mathrm{~N}}\end{array}$ & $\begin{array}{c}0 \\
\stackrel{\omega}{n}\end{array}$ & $\begin{array}{c}0 \\
\stackrel{\sin }{\mathrm{N}}\end{array}$ & $\begin{array}{c}0 \\
\text { ஸे } \\
\text { | }\end{array}$ & $\begin{array}{c}0 \\
\stackrel{1}{N}\end{array}$ & $\begin{array}{c}0 \\
\stackrel{\omega}{N}\end{array}$ & $\begin{array}{c}0 \\
\stackrel{\omega}{N}\end{array}$ & $\begin{array}{l}0 \\
\stackrel{\circ}{N}\end{array}$ & 일 & $\begin{array}{l}0 \\
\stackrel{N}{N}\end{array}$ & $\begin{array}{c}0 \\
\stackrel{1}{N} \\
\mid\end{array}$ & & $\begin{array}{l}\text { 울 } \\
\text { مै }\end{array}$ \\
\hline 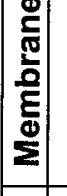 & 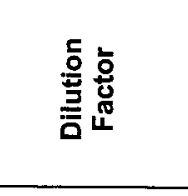 & $\begin{array}{l}8 \\
8 \\
8 \\
8\end{array}$ & $\begin{array}{l}8 \\
8 \\
8 \\
8\end{array}$ & $\begin{array}{l}8 \\
8 \\
8 \\
8\end{array}$ & 요 & 으 & $\stackrel{ }{\square}$ & $ㅇ$ & 유 & 우 & $\begin{array}{l}8 \\
8 \\
8 \\
8\end{array}$ & $\begin{array}{l}8 \\
8 \\
8 \\
8\end{array}$ & $\begin{array}{l}8 \\
8 \\
8 \\
8\end{array}$ & & 음 \\
\hline & ろ总苍 & 0 & 0 & 0 & \& & P & 암 & 8 & 8 & 8 & 0 & 0 & 0 & & g \\
\hline & 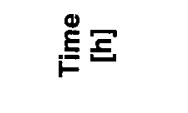 & 8 & & & $\stackrel{8}{\circ}$ & & & $\stackrel{\circ}{\circ}$ & & & مَ & & & & $\begin{array}{l}\infty \\
\infty \\
\infty \\
\infty\end{array}$ \\
\hline
\end{tabular}


F

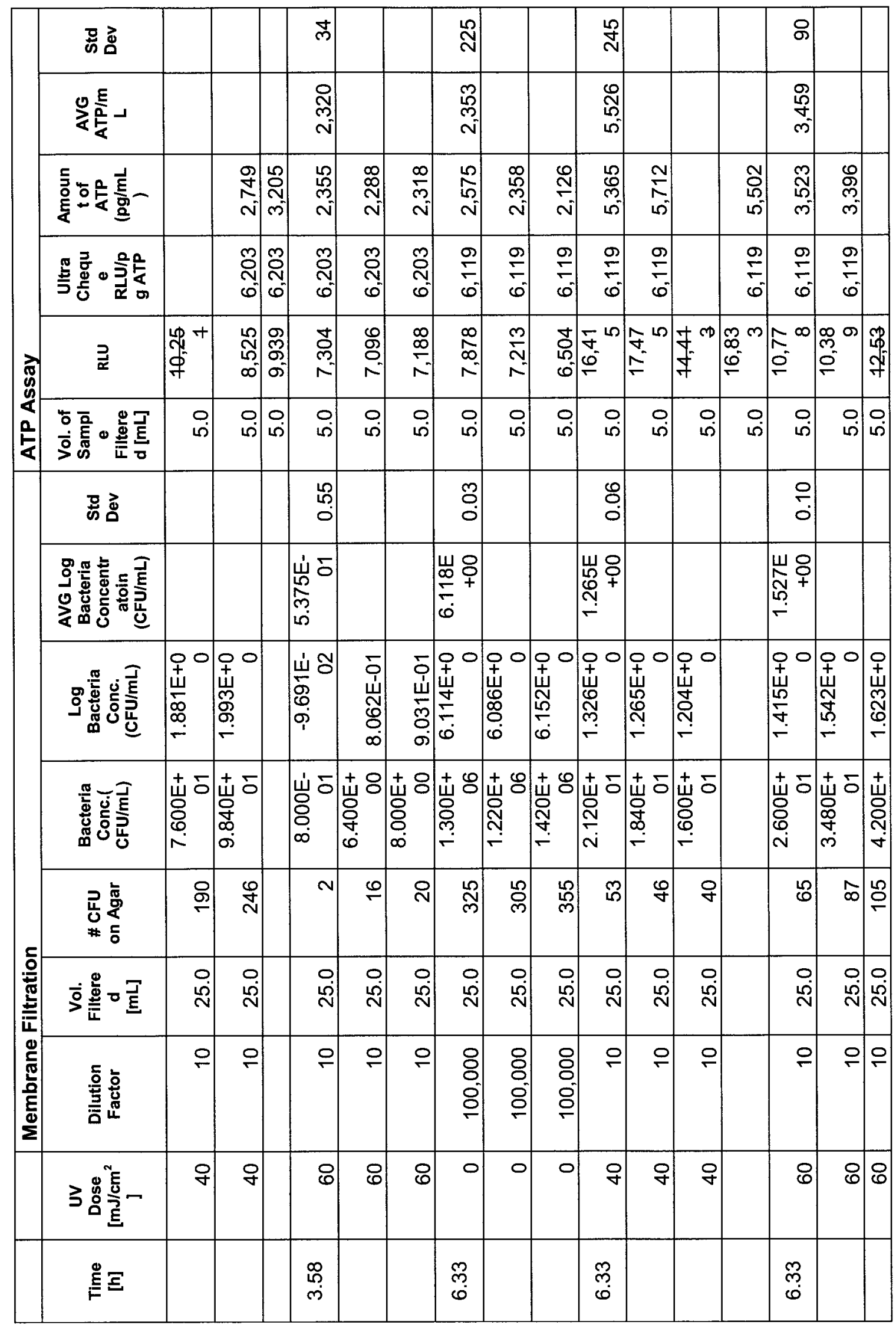


ㄱำ

\begin{tabular}{|c|c|c|c|c|c|c|c|c|c|c|c|c|}
\hline 离高 & & 电 & & & \begin{tabular}{l|l}
$\stackrel{\infty}{\infty}$ \\
$\stackrel{2}{*}$
\end{tabular} & & & $\frac{7}{4}$ & & & $\bar{\infty}$ & \\
\hline 晏镸 & & : & & & हैं & & & : & & & స్̄ं & \\
\hline 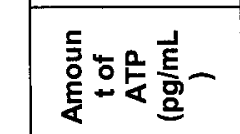 & & 照 & 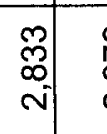 & , & 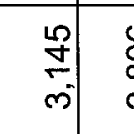 & 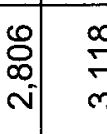 & & & 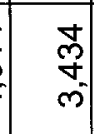 & & 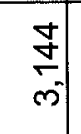 & 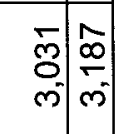 \\
\hline 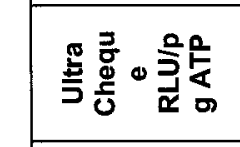 & & 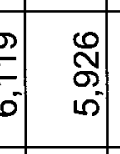 & 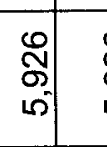 & (i) & 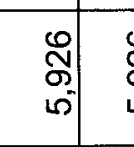 & 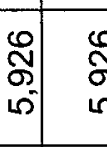 & & & 总 & 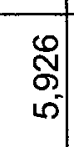 & $\begin{array}{l}0 \\
0 \\
0 \\
6 \\
6\end{array}$ & 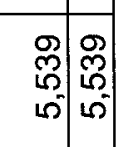 \\
\hline$\underline{\underline{z}}$ & $M \stackrel{=}{=}$ & $\left|\begin{array}{c|}0 \\
0 \\
0 \\
\infty \\
\infty\end{array}\right|$ & 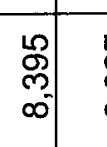 & 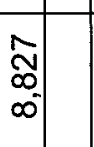 & \begin{tabular}{c|c}
0 \\
$\vdots$ \\
0 \\
0
\end{tabular} & 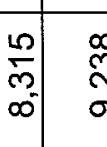 & & 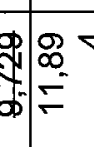 & & & $\begin{array}{l}0 \\
\\
\infty \\
\infty\end{array}$ & 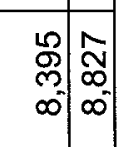 \\
\hline 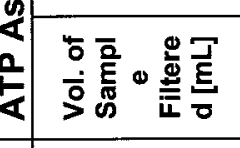 & & is & iी & iी & 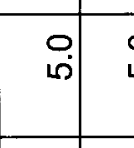 & 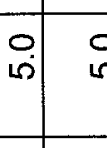 & & $6:$ & is & î| & is & 60 \\
\hline 蚫高 & & 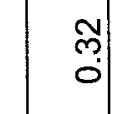 & & & :. & & 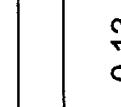 & ?ִ. & & & & \\
\hline 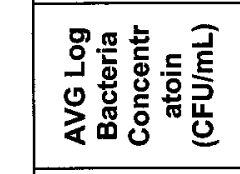 & & 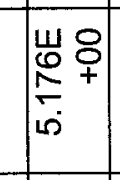 & & & 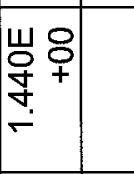 & & 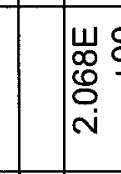 & & & & & \\
\hline 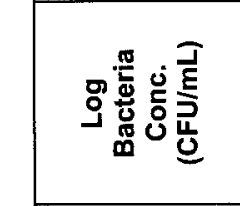 & & 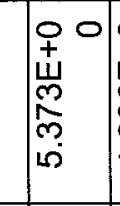 & $\begin{array}{l}0 \\
0 \\
0\end{array}$ & & 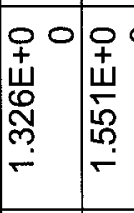 & & & ol & & & & \\
\hline 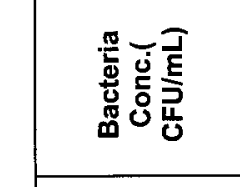 & & 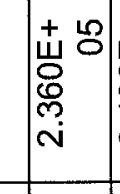 & & & 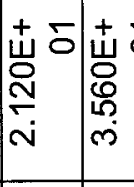 & & & 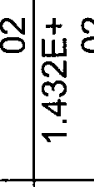 & & & & \\
\hline 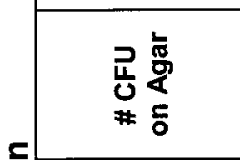 & & of & 6 & 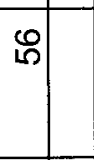 & की & $D 8$ & & & i & & & o \\
\hline 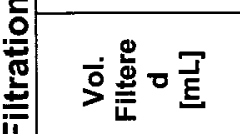 & & ㅇํㅇ & ڤ̊ํ: & $\stackrel{\circ}{\dot{s}}$ & ڤึ่ & 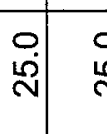 & & 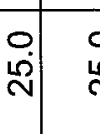 & id & & 然 & เุ่ำ \\
\hline 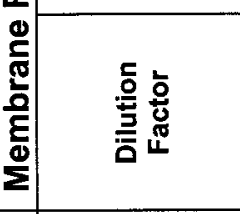 & & $\begin{array}{l}0 \\
0 \\
0\end{array}$ & $\begin{array}{l}0 \\
\vdots \\
\vdots \\
\vdots\end{array}$ & : & 우 & $\circ:$ & & $\circ:$ & 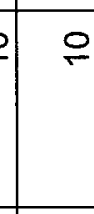 & & & : \\
\hline 3总言- & & 0 & 0 & 0 & $q$ & 89 & & & 8 & & & \\
\hline 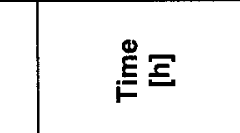 & & $\stackrel{8}{\circ}$ & & & \begin{tabular}{|l|} 
\\
0 \\
0
\end{tabular} & & c & s: & & & 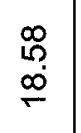 & \\
\hline
\end{tabular}




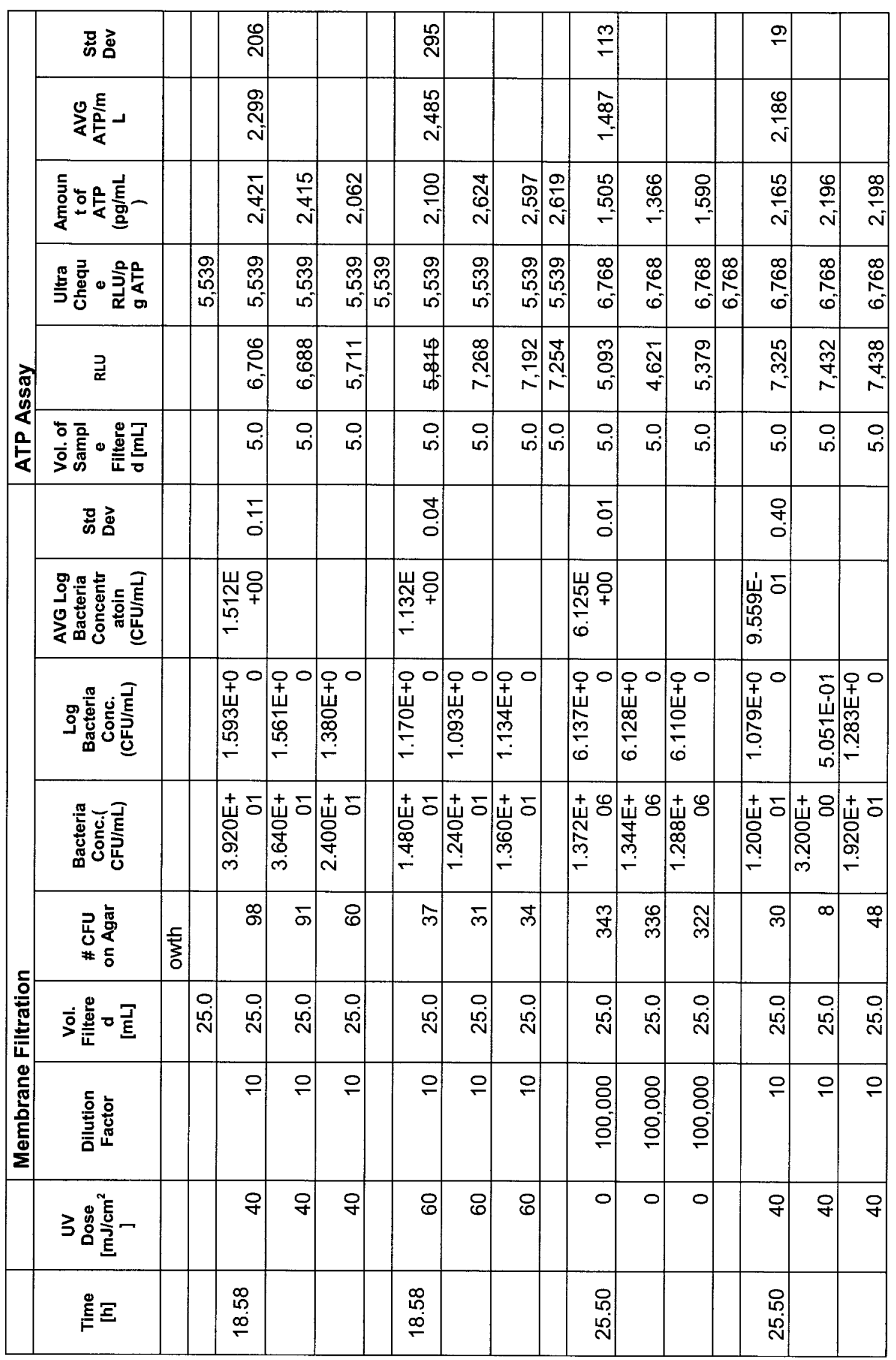


素

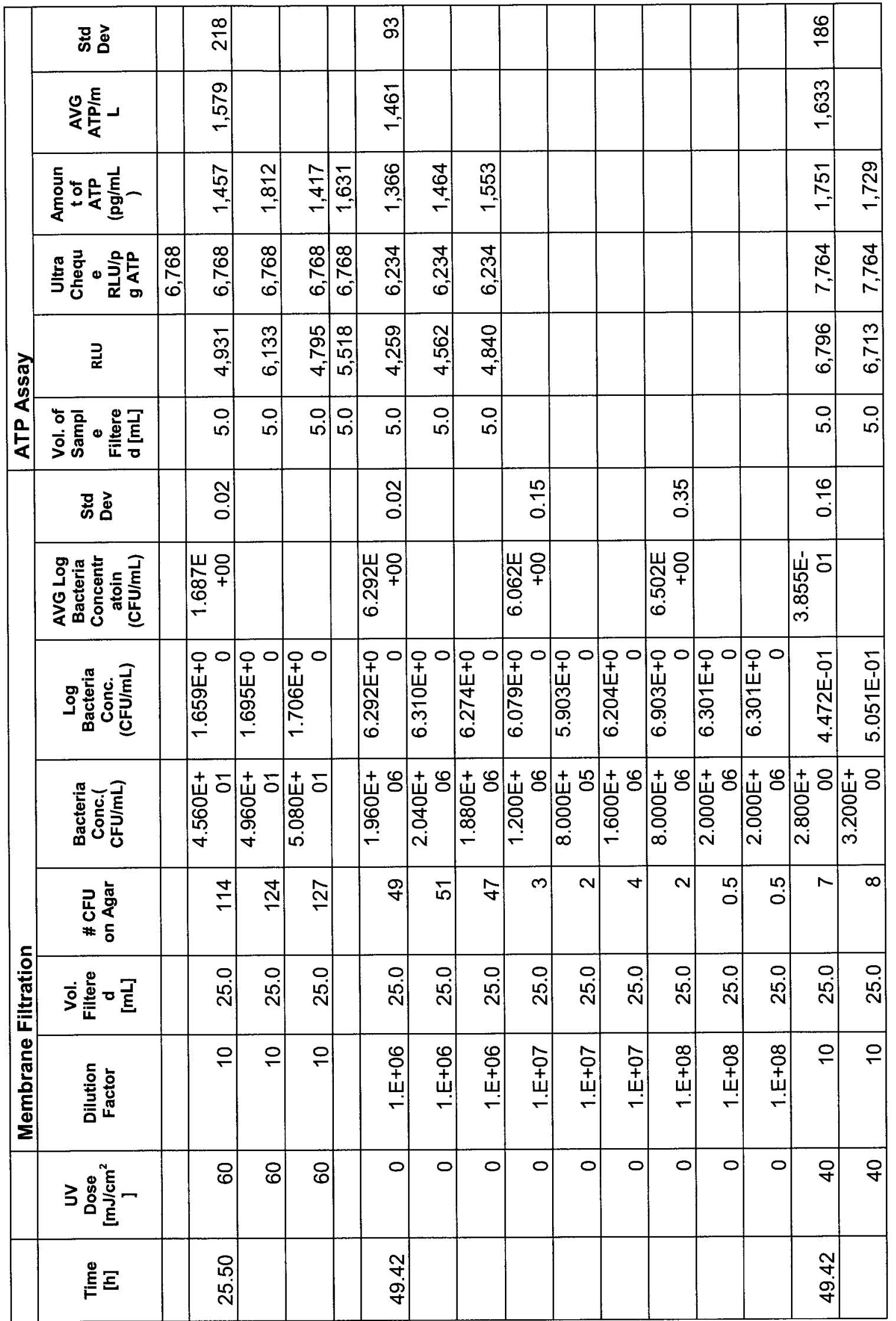


$\stackrel{n}{\sim}$

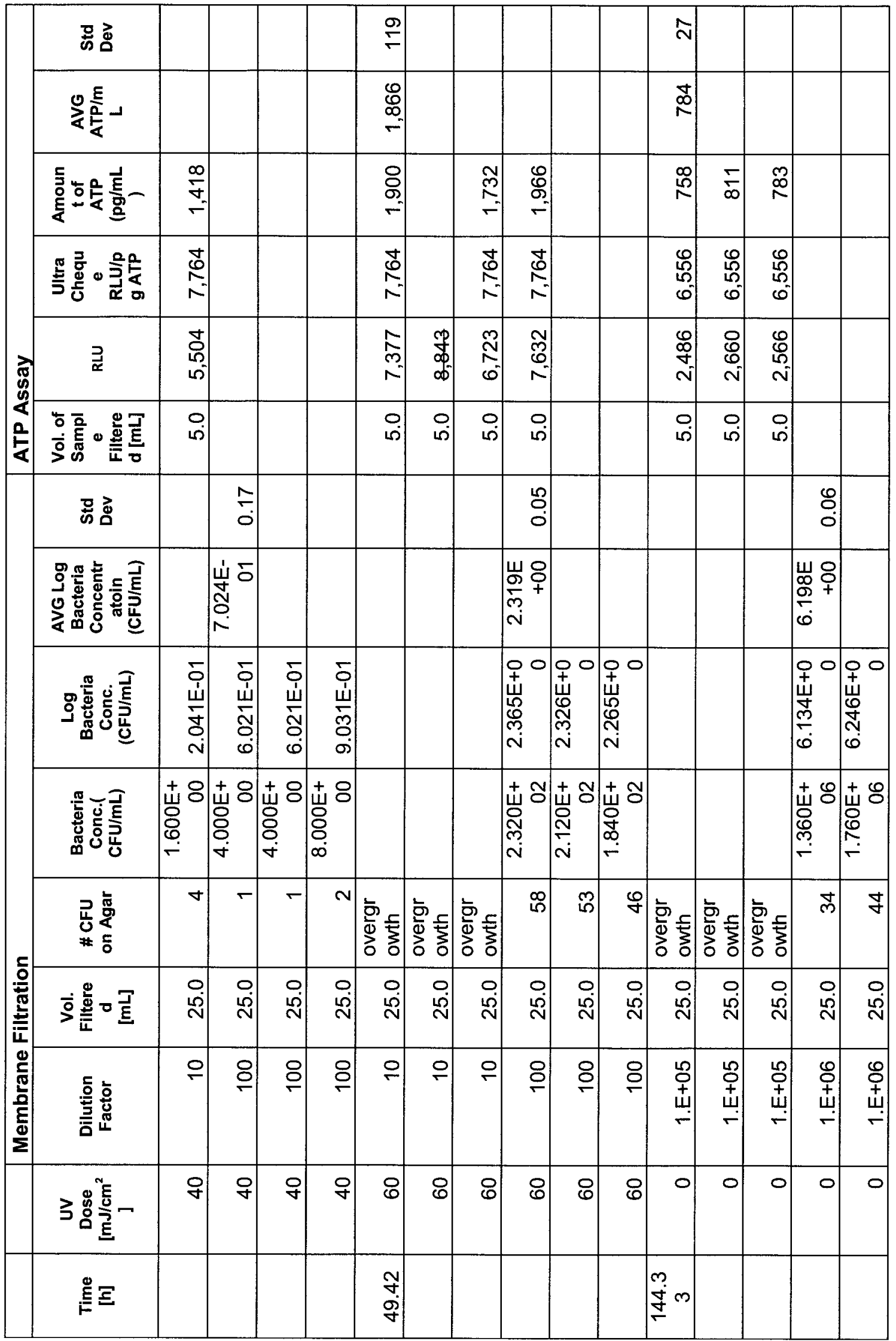


워 군

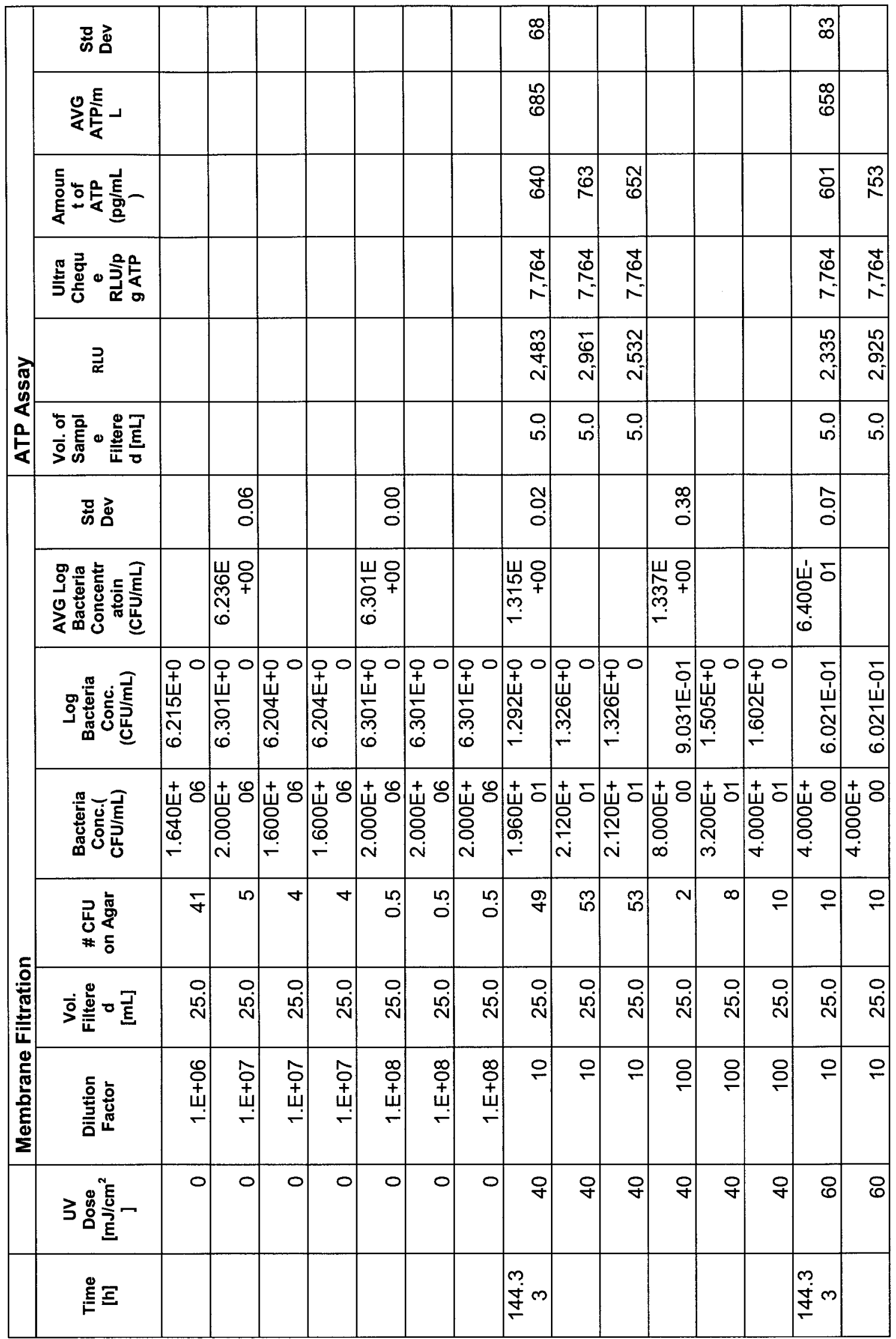


‡

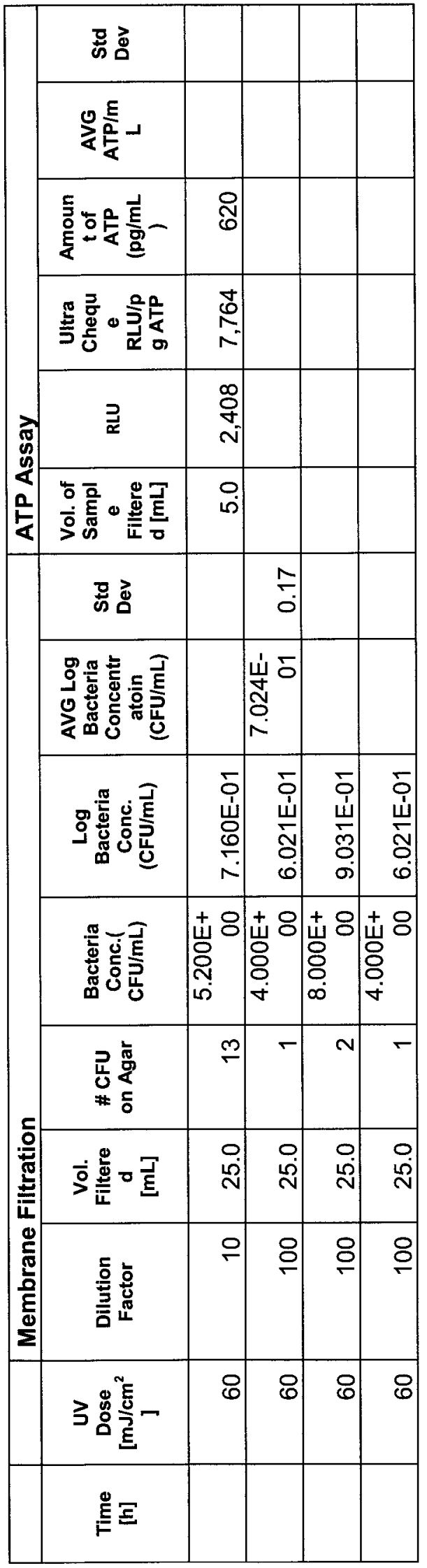

\begin{tabular}{|c|c|c|c|c|c|c|c|c|}
\hline 焉 & 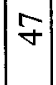 & & & ले & & & F & \\
\hline$\stackrel{0}{\frac{g}{g}}$ & $\left|\begin{array}{c}9 \\
+\infty\end{array}\right|$ & & & $\mid \begin{array}{l}n \\
n \\
-\end{array}$ & & & $\underset{\widetilde{\sigma}}{\sim}$ & \\
\hline$\frac{\vec{E}}{\frac{E}{R}}$ & ڤ్ & $\stackrel{\infty}{\infty}$ & $\underset{\infty}{\infty}$ & $\left|\begin{array}{l}0 \\
0 \\
0 \\
-1\end{array}\right|$ & $\bar{n}$ & ब్ & f & 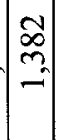 \\
\hline$\stackrel{\vec{\not}}{\partial}$ & $\begin{array}{l}n \\
\vec{\gamma} \\
\sim \\
\sim\end{array}$ & $\frac{N}{N}$ & $\stackrel{\infty}{\infty}$ & $\begin{array}{l}0 \\
10 \\
5 \\
7\end{array}$ & $\mid \begin{array}{l}n \\
\tilde{a} \\
f\end{array}$ & $\stackrel{\hat{n}}{m}$ & $\begin{array}{l}N \\
\infty \\
\infty \\
m\end{array}$ & $\mid \begin{array}{l}0 \\
8 \\
0 \\
m\end{array}$ \\
\hline
\end{tabular}

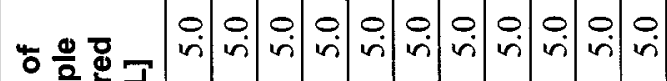

\begin{tabular}{|c|c|c|c|c|c|c|c|c|c|}
\hline \multicolumn{10}{|l|}{ 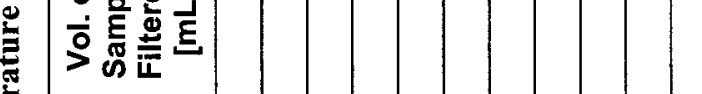 } \\
\hline $\begin{array}{l}\text { Dे } \\
\text { के }\end{array}$ & : & & 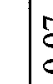 & & & $\stackrel{8}{0}$ & & & $\begin{array}{c}m \\
0 \\
\end{array}$ \\
\hline$\stackrel{9}{8}$ & $\left|\begin{array}{c}0 \\
n \\
n \\
m\end{array}\right|$ & & 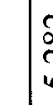 & & & \begin{tabular}{l}
\multirow{2}{\$}{} \\
$\infty$ \\
0 \\
0
\end{tabular} & & & के. \\
\hline ن & $\mid \begin{array}{c}\mathbb{S} \\
\text { n̦ } \\
\dot{m}\end{array}$ & 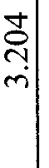 & के & 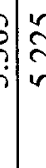 & 年 & 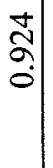 & 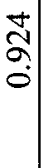 & $\begin{array}{l}m \\
\hat{\infty} \\
0\end{array}$ & $\begin{array}{l}\checkmark \\
\dot{y} \\
\dot{0}\end{array}$ \\
\hline$\frac{\vec{\xi}}{\vec{z}}$ & $\mid \begin{array}{c}0 \\
0 \\
1 \\
\text { 至 } \\
0 \\
-1\end{array}$ & $\begin{array}{c}0 \\
0 \\
+ \\
0 \\
0 \\
0 \\
-\end{array}$ & 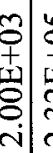 & 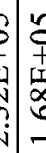 & 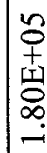 & $\begin{array}{c}8 \\
8 \\
+ \\
\text { 甲 } \\
8 \\
+ \\
\infty\end{array}$ & \begin{tabular}{c}
8 \\
8 \\
+ \\
甲্ \\
\multirow{8}{+}{} \\
$\infty$
\end{tabular} & 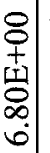 & 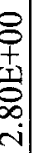 \\
\hline 吾 & $\nabla$ & $\nabla$ & $n:$ & $0 \%$ & $q$ & $\vec{\sim}$ & $\bar{N}$ & $\triangle$ & $\pi$ \\
\hline 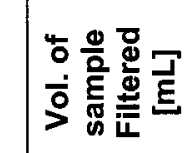 & $\approx 2$ & $\approx$ & $\approx$ & 3 & $\approx$ & $\approx$ & $\approx$ & $\approx$ & $\approx$ \\
\hline 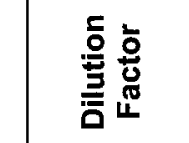 & $\mid \begin{array}{l} \pm \\
0 \\
+ \\
+ \\
-\end{array}$ & $\begin{array}{l} \pm \\
+ \\
+ \\
\\
-\end{array}$ & 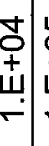 & بأ. & $\begin{array}{l}0 \\
0 \\
+ \\
1 \\
-\end{array}$ & - & 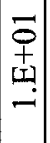 & 古 & - \\
\hline 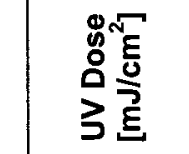 & 0 & 0 & 0 & 010 & 0 & 암 & 암 & 이 & 워 \\
\hline 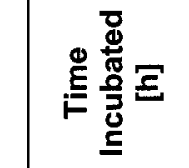 & $\mid \begin{array}{l}8 \\
0 \\
0\end{array}$ & & & & & $\mid \begin{array}{l}8 \\
0 \\
0\end{array}$ & & & ن \\
\hline
\end{tabular}




\begin{tabular}{|c|c|c|c|c|c|c|c|}
\hline 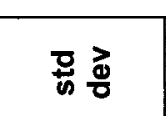 & $\mid$ 可 & $|\infty|$ & 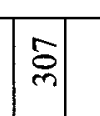 & 1 & $\overline{\mid} \mid \bar{f}$ & & 嫼 \\
\hline 举㖘 & 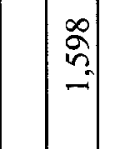 & 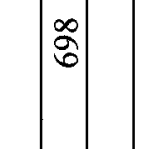 & 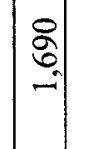 & 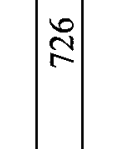 & ;: & & $\left|\frac{\frac{n}{x}}{x_{i}}\right|$ \\
\hline 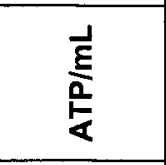 & 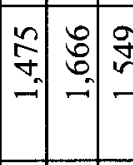 & & & & 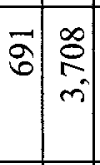 & & 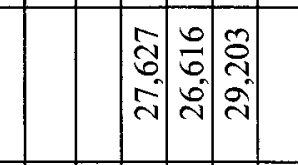 \\
\hline$\underline{z}$ & 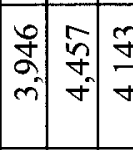 & 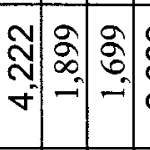 & $\tau$ & & : & & 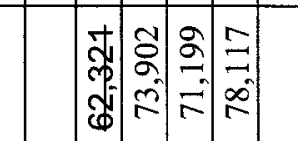 \\
\hline 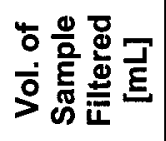 & & & & & 5 & & \\
\hline 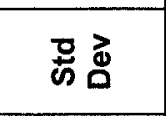 & : & 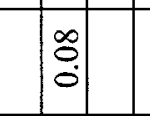 & 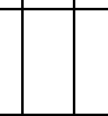 & $\frac{5}{6}$ & 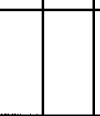 & & \\
\hline$\frac{⿱}{8}$ & : & : & 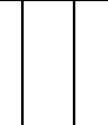 & | & 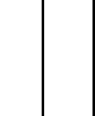 & & \\
\hline 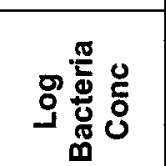 & 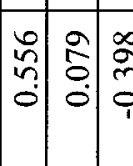 & 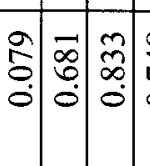 & & $\mid$ & & & \\
\hline 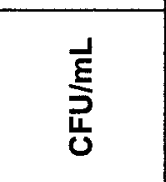 & 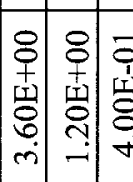 & 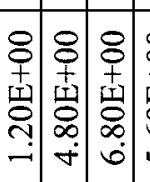 & & & & & \\
\hline 旁高 & & & & & & & \\
\hline 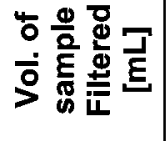 & & & & & & & \\
\hline 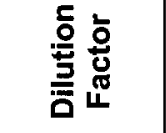 & 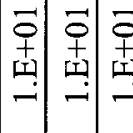 & & & & 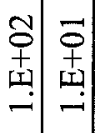 & & 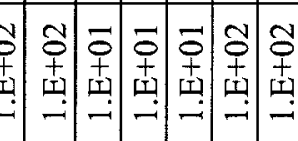 \\
\hline 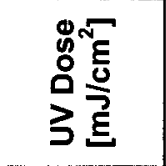 & & 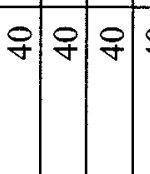 & & & $F$ F & & \\
\hline 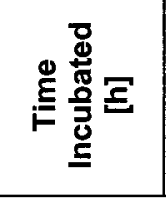 & 9 & $\stackrel{8}{\infty}$ & 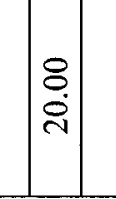 & 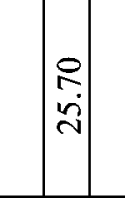 & 年 & & $\frac{n}{n}$ \\
\hline
\end{tabular}


웜

\begin{tabular}{|c|c|c|c|c|c|c|c|c|c|c|}
\hline 호 & & $\mid \begin{array}{c}|c| \\
6 \\
q\end{array}$ & & & & & & & & \\
\hline$\underset{\frac{Q}{2}}{0}$ & & $\left|\begin{array}{c}8 \\
8 \\
\infty\end{array}\right|$ & & & & & & & & \\
\hline 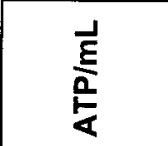 & & \begin{tabular}{|l|} 
\\
2 \\
$\infty$
\end{tabular} & & 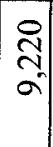 & $\begin{array}{l}\hat{\Omega} \\
\text { s. } \\
\infty\end{array}$ & & & & & \\
\hline 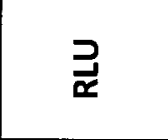 & & $\begin{array}{l}m \\
\tilde{n} \\
\hat{n}\end{array}$ & 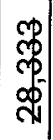 & $\begin{array}{l}0 \\
b \\
\dot{0}\end{array}$ & 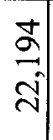 & & & & & \\
\hline 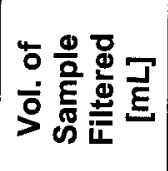 & & $\begin{array}{c}0 \\
\dot{n}\end{array}$ & $\begin{array}{c}0 \\
\dot{r}\end{array} \mid$ & $\begin{array}{c}0 \\
\text { in }\end{array}$ & $\begin{array}{l}0 \\
\dot{n}\end{array}$ & & & & & \\
\hline कृ す & & & & & & & & & & \\
\hline$\stackrel{9}{\frac{9}{\alpha}}$ & & & & & & & & & & \\
\hline ن & & & & & & & & & & \\
\hline$\frac{1}{\xi}$ & & & & & & & & & & \\
\hline 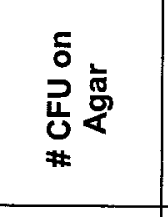 & 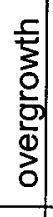 & 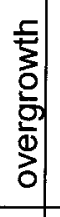 & 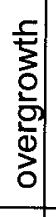 & 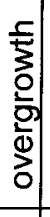 & 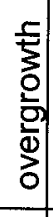 & 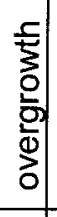 & 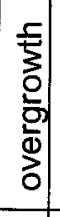 & \begin{tabular}{|c|}
5 \\
$\vdots$ \\
0 \\
0 \\
0 \\
0 \\
0 \\
\end{tabular} & \begin{tabular}{|c|}
$\frac{5}{3}$ \\
0 \\
0 \\
0 \\
0 \\
0
\end{tabular} & \begin{tabular}{|l|} 
\\
$\vdots$ \\
0 \\
0 \\
0 \\
0 \\
0 \\
\end{tabular} \\
\hline 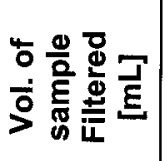 & & $\approx$ & 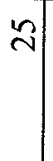 & $\approx$ & 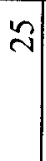 & $\approx$ & $\approx$ & & $\approx$ & 2 \\
\hline 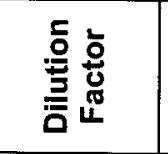 & 点 & 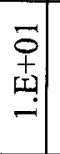 & 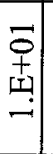 & $\begin{array}{c} \\
0 \\
+ \\
-1 \\
-1\end{array}$ & 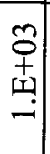 & 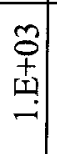 & $\begin{array}{l} \\
0 \\
0 \\
1 \\
1 \\
-1\end{array}$ & $\begin{array}{l} \\
0 \\
\pm \\
\text { 至 } \\
-\end{array}$ & 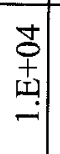 & 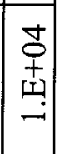 \\
\hline 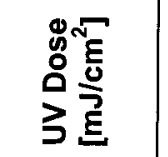 & & $q$ & F & ㅇ & $\mathcal{F}$ & q & 아 & 아 & 우 & f \\
\hline 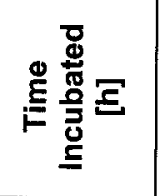 & & $\begin{array}{l}n \\
\mathfrak{j} \\
\dot{v}\end{array}$ & & & & & & & & \\
\hline
\end{tabular}

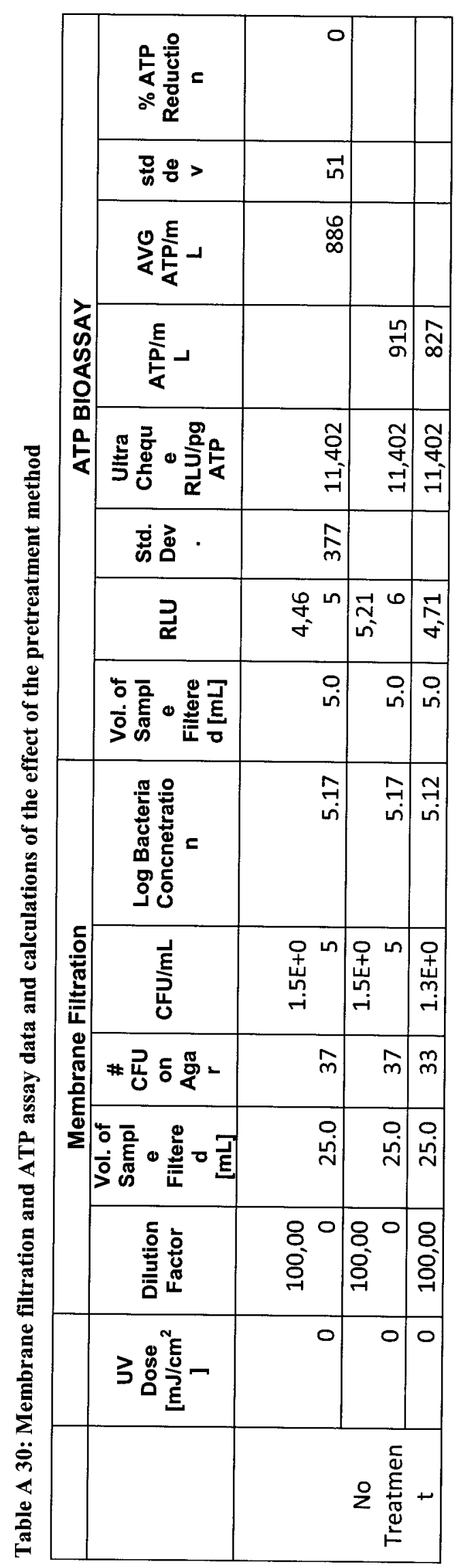




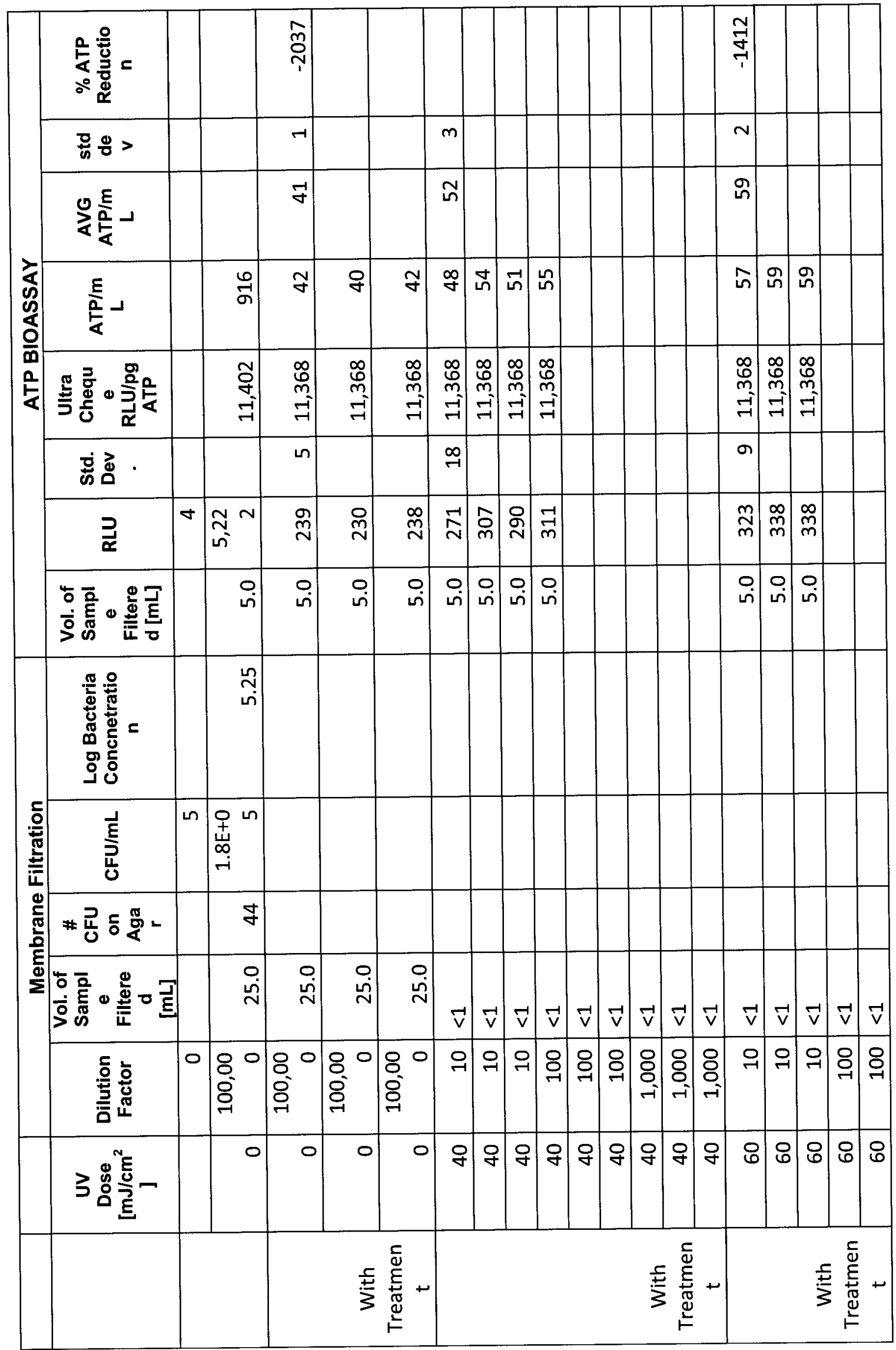


$\vec{a}$

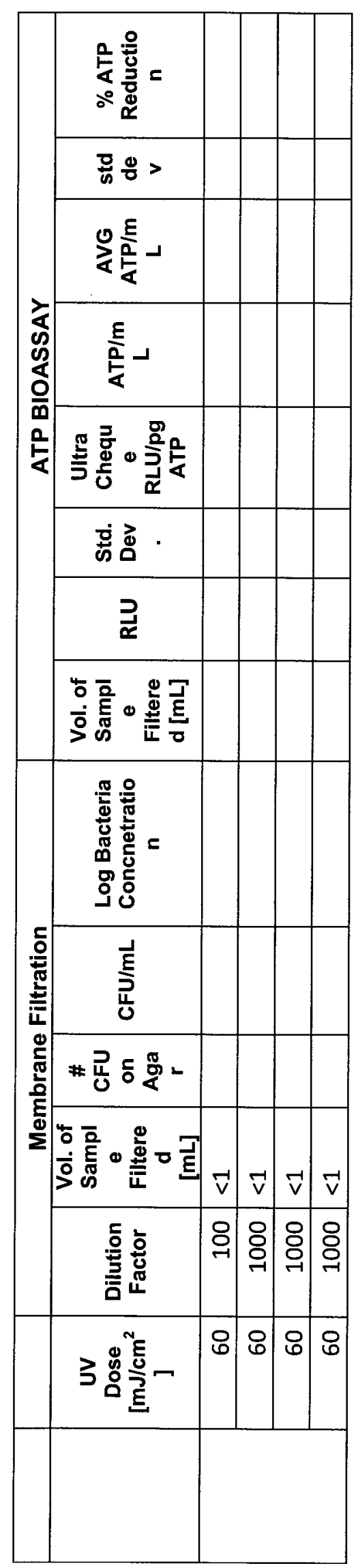




\begin{tabular}{|c|c|c|c|c|c|c|}
\hline & 产高 & तो & $\vec{N}$ & f & $\approx$ & $\pi$ \\
\hline & $\frac{9}{4}$ & : & $\mid$ चि & 要 & \begin{tabular}{|l|l|} 
pof \\
\end{tabular} & t \\
\hline & 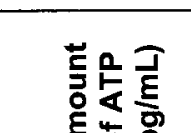 & 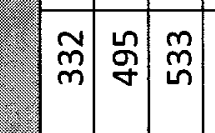 & 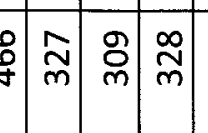 & 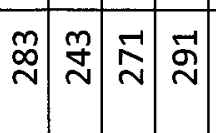 & 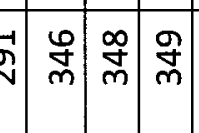 & 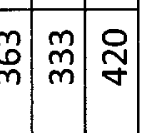 \\
\hline & 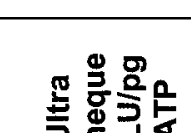 & & . & 赵: & & تص \\
\hline & $\overrightarrow{\underline{u}}$ & 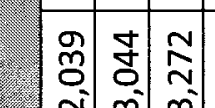 & $8:$ & qี & 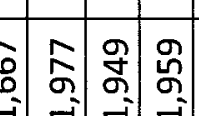 & : \\
\hline & 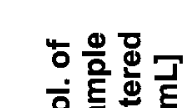 & & 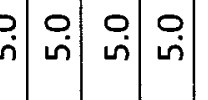 & $|i|$ & & : \\
\hline & & & & & & \\
\hline & 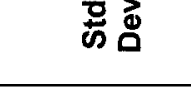 & : & : & 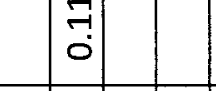 & & : \\
\hline & $\frac{8}{8}$ & 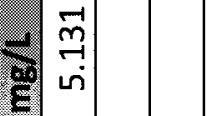 & $\mid$ & $\mid$ & & 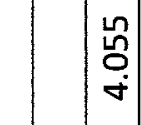 \\
\hline & 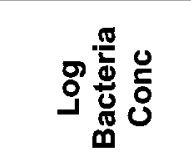 & 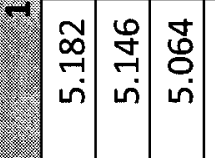 & 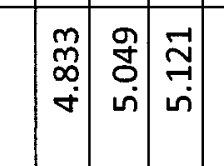 & 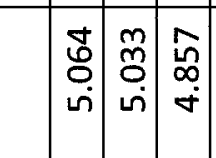 & & 8 \\
\hline & 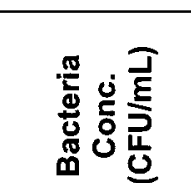 & 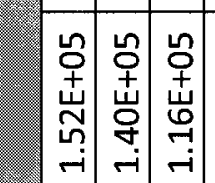 & 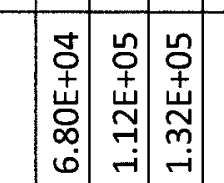 & : & & | \\
\hline & 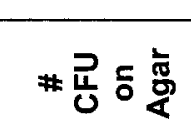 & 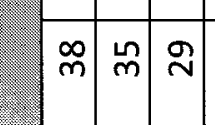 & $\Rightarrow \approx \tilde{N}$ & $\tilde{N} \approx \sim$ & & 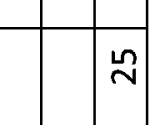 \\
\hline & 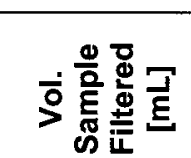 & & 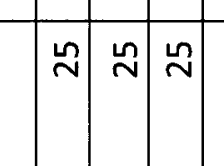 & $\stackrel{\sim}{\infty}$ & & 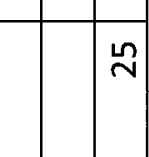 \\
\hline & 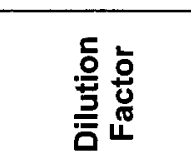 & 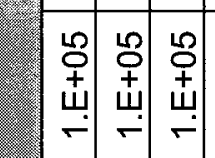 & 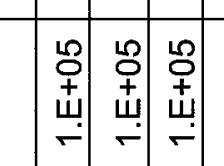 & 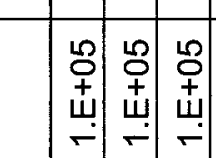 & & 茧 \\
\hline & 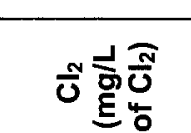 & $\mid \begin{array}{c}0 \\
0\end{array}$ & $:$ & $\mid$ & $\ddot{0}$ & శึ \\
\hline & 毫焉 & $\circ$ & $n$ & 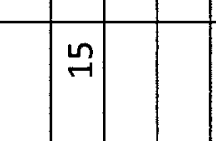 & i & 7 \\
\hline
\end{tabular}




\begin{tabular}{|c|c|c|c|c|c|c|}
\hline 紊高 & & & $\tilde{N}$ & n & $=$ & $\approx$ \\
\hline$\frac{z}{8}$ & & & สิ & $\mathbb{\Xi}$ & 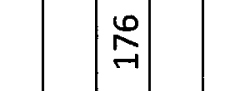 & $\Phi$ \\
\hline 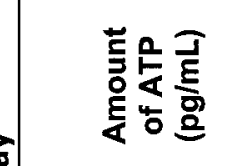 & & & 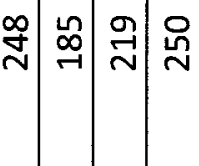 & 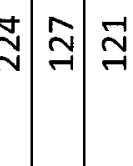 & 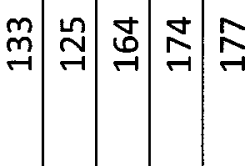 & $\mathrm{AO}$ \\
\hline 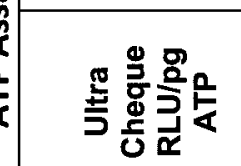 & & & & & & \\
\hline$\vec{z}$ & 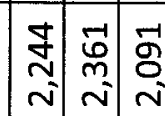 & 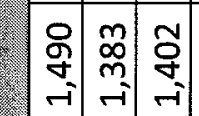 & 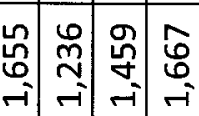 & 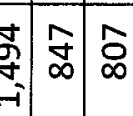 & 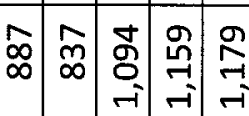 & 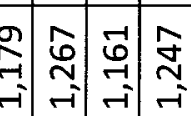 \\
\hline 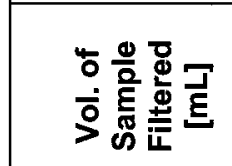 & & & 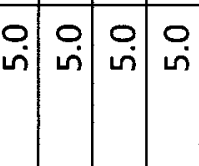 & 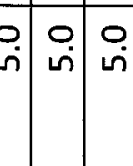 & 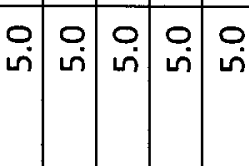 & 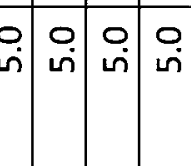 \\
\hline 䇏育 & & 10 & : & 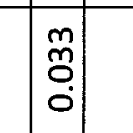 & | & 思 \\
\hline$\frac{9}{8}$ & & 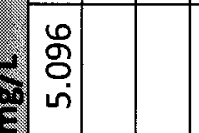 & 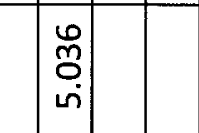 & $\mid$ & 蓆 & $\mid \begin{array}{l}\mid \pi \\
\tilde{z}\end{array}$ \\
\hline 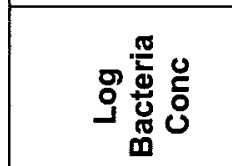 & $\mid$ & 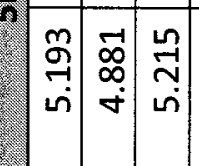 & $\frac{7}{2}: 0$ & 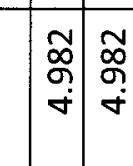 & 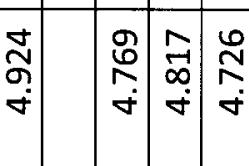 & \begin{tabular}{l|l}
8 \\
\hdashline
\end{tabular} \\
\hline 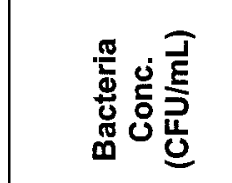 & | & 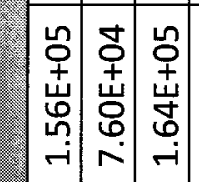 & 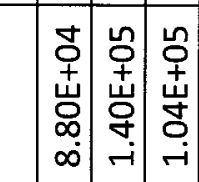 & & 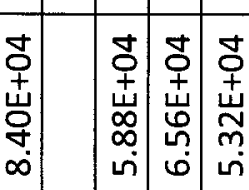 & 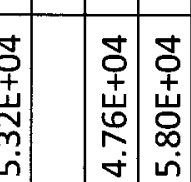 \\
\hline 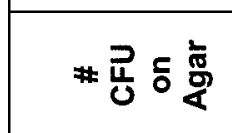 & $\infty$ & $\begin{array}{l}9 \\
\Rightarrow\end{array}$ & $\pi$ तथी & & 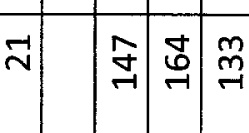 & 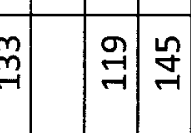 \\
\hline 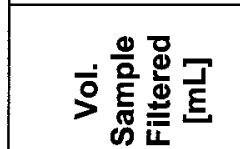 & & & & & $\stackrel{\sim}{\sim}$ & \\
\hline 譬产 & & 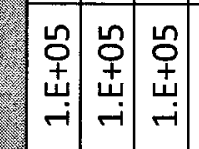 & 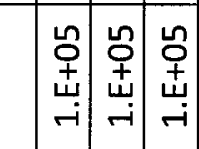 & 节 & 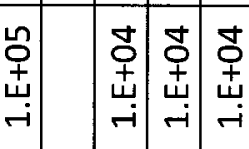 & 怘怘 \\
\hline 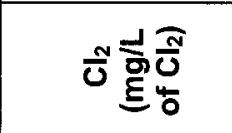 & & $\frac{0}{i s}$ & 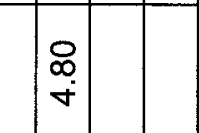 & $\mid$ & q & $\left|\begin{array}{l}0 \\
0 \\
\infty\end{array}\right|$ \\
\hline 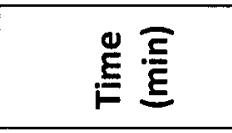 & & & n & ต & if & yी \\
\hline
\end{tabular}




\begin{tabular}{|c|c|c|c|c|c|c|c|c|c|c|c|c|c|c|c|c|c|c|c|c|c|c|}
\hline & 密宫 & & & $\ddot{\emptyset}$ & & & & \& & & & & ৪ & & & & $\underset{\sim}{\infty}$ & & & & g & & \\
\hline & $\frac{9}{8}$ & & & $\stackrel{6}{\circ}$ & & & & ָั & & & & 宇 & & & & $\left|\begin{array}{l}\infty \\
0 \\
\sigma\end{array}\right|$ & & & & ஸั & & \\
\hline 入 & 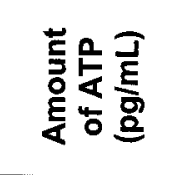 & 只 & 이: & $\tilde{m}$ & $\mid \begin{array}{l}0 \\
0 \\
-1 \\
-1\end{array}$ & $\stackrel{\infty}{+}$ & 8 & 은 & 冓 & $\stackrel{\infty}{\stackrel{\sim}{\sigma}}$ & นึ & న్ & กิ & $\underset{\mathcal{Y}}{\mathbb{Z}}$ & 곤 & $\tilde{N}$ & 离 & $\begin{array}{l}\infty \\
0 \\
\forall\end{array}$ & $\stackrel{n}{\underset{\forall}{\sigma}}$ & ১) & సิ & \\
\hline 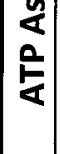 & 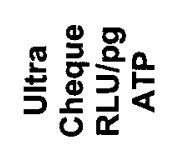 & 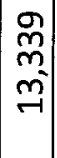 & $\begin{array}{l}\stackrel{a}{m} \\
m \\
m \\
\vec{\sim}\end{array}$ & $\begin{array}{l}\curvearrowright \\
\infty \\
\sigma\end{array}$ & \begin{tabular}{l}
\multirow{2}{0}{} \\
$\infty$ \\
0
\end{tabular} & 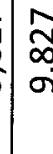 & $\frac{1}{2}$ & $\begin{array}{l}\hat{\jmath} \\
\vdots \\
0 \\
0\end{array}$ & $\begin{array}{l}\hat{N} \\
\infty \\
\sigma\end{array}$ & $\begin{array}{l}\hat{\sim} \\
\infty \\
\text { के }\end{array}$ & $\begin{array}{l}\hat{N} \\
\infty \\
\sigma\end{array}$ & స్ & $\begin{array}{c}\hat{\lambda} \\
\infty \\
\sigma\end{array} \mid$ & $\begin{array}{l}\hat{N} \\
\infty \\
\sigma\end{array}$ & $\mid$ & $\begin{array}{l}\widehat{N} \\
\infty \\
\sigma\end{array}$ & $\begin{array}{c}\text { D } \\
\infty \\
\sigma \\
\sigma\end{array}$ & $\begin{array}{l}\widehat{a} \\
\infty \\
\sigma\end{array}$ & \begin{tabular}{l|l} 
& 1 \\
$\infty$ & \\
के &
\end{tabular} & \begin{tabular}{l|l}
$\hat{A}$ & 1 \\
$\infty$ & 2 \\
$\sigma$ & 0
\end{tabular} & 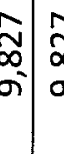 & $\begin{array}{l}\text { s } \\
\infty \\
\text { o' }\end{array}$ \\
\hline & $\stackrel{コ}{\not \supset}$ & $\begin{array}{c}\vec{m} \\
m \\
r i\end{array}$ & $\underset{m}{m}$ & $\begin{array}{l}5 \\
0 \\
0\end{array}$ & \% & 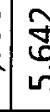 & I & 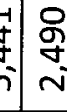 & $\frac{-1}{2}$ & \begin{tabular}{l}
$\infty$ \\
\multirow{m}{m}{} \\
$\sim$ \\
$\sim$
\end{tabular} & $\begin{array}{l}0 \\
6 \\
i \\
N\end{array}$ & 早 & $\left|\begin{array}{l}0 \\
m \\
r \\
-i\end{array}\right|$ & $\mid \begin{array}{c}n \\
\vdots \\
i \\
n\end{array}$ & $\begin{array}{c}0 \\
5 \\
-i \\
-i\end{array}$ & $\begin{array}{c}n \\
6 \\
6 \\
N\end{array}$ & స్ & 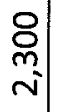 & \begin{tabular}{l|l}
$\infty$ & \\
& \\
$\sim$ &
\end{tabular} & 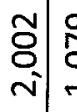 & $\begin{array}{c}0 \\
6 \\
-i\end{array}$ & \\
\hline & 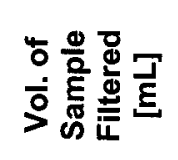 & $\begin{array}{l}0 \\
\text { in }\end{array}$ & 이 & c & in & in & S & in & 옹 & 우 & 우 & 웅 & $\begin{array}{l}0 \\
\text { in }\end{array}$ & $\begin{array}{c}0 \\
\text { in }\end{array}$ & 이 & 임 & 이 & $\begin{array}{c}0 \\
\text { in }\end{array}$ & 인 & \begin{tabular}{l|l}
0 \\
ن.
\end{tabular} & S. & \\
\hline \multirow{9}{*}{ 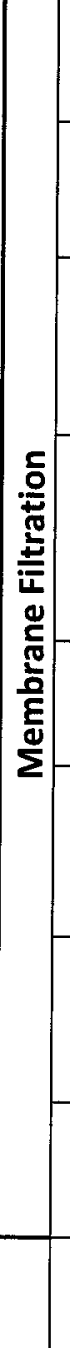 } & 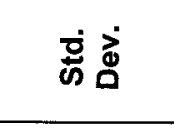 & & & $\begin{array}{l}\bar{z} \\
\bar{c} \\
\end{array}$ & & & & ๙̃ & & & & \& & & & & $\begin{array}{c}+ \\
\stackrel{-}{-} \\
0\end{array}$ & & & & $\begin{array}{l}8 \\
8 \\
0 \\
\vdots\end{array}$ & & \\
\hline & 定 & & & 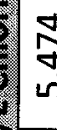 & & & & 豆 & & & & 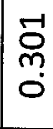 & & & & $\begin{array}{c}-1 \\
\stackrel{\sigma}{\sigma} \\
\dot{0}\end{array}$ & & & & $\begin{array}{l}\text { Oे } \\
6 \\
0 \\
\end{array}$ & & \\
\hline & ن & $\mid \begin{array}{l}\stackrel{0}{N} \\
\stackrel{\nabla}{\sim}\end{array}$ & & $\frac{7}{\partial}$ & $\stackrel{\sim}{\dddot{n}}$ & $\begin{array}{l}6 \\
\text { जn } \\
\text { นn }\end{array}$ & & 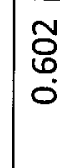 & î & is & & 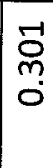 & $\begin{array}{l}-1 \\
0 \\
0 \\
0\end{array}$ & $\begin{array}{c}-1 \\
\text { m. } \\
0\end{array}$ & & 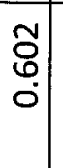 & \begin{tabular}{c}
-1 \\
\multicolumn{1}{c}{} \\
0 \\
0
\end{tabular} & $\begin{array}{l}-1 \\
\stackrel{m}{0} \\
0\end{array}$ & & $\begin{array}{l}9 \\
0 \\
0 \\
0\end{array}$ & $\begin{array}{l}9 \\
9 \\
0 \\
0 \\
\dot{1}\end{array}$ & 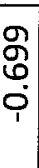 \\
\hline & 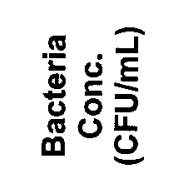 & 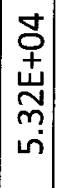 & & 岁 & 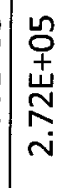 & $\begin{array}{l}\text { L } \\
0 \\
+ \\
\infty \\
\infty \\
m\end{array}$ & & $\begin{array}{l}8 \\
\stackrel{+}{+} \\
\stackrel{4}{8} \\
\stackrel{8}{+}\end{array}$ & 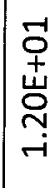 & $\begin{array}{l}8 \\
0 \\
+ \\
\text { 岁 } \\
\dot{1}\end{array}$ & & 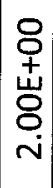 & 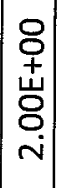 & 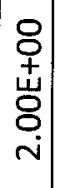 & & $\begin{array}{l} \\
\\
+ \\
\text { 山े } \\
\dot{f} \\
\dot{f}\end{array}$ & $\begin{array}{l}8 \\
0 \\
+ \\
\stackrel{+}{0} \\
\dot{v}\end{array}$ & 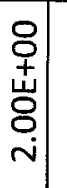 & & 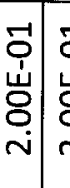 & $\begin{array}{l}-1 \\
0 \\
1 \\
\dot{1} \\
8 \\
\dot{0} \\
ن\end{array}$ & $\begin{array}{l}-1 \\
0 \\
\dot{u} \\
0 \\
\dot{0} \\
\text { í }\end{array}$ \\
\hline & * 峲 & $\stackrel{m}{m}$ & & $\stackrel{N}{N}$ & $\ddot{0}$ & $\approx$ & & - & $m$ & In & & $\stackrel{4}{0}$ & \begin{tabular}{|c|} 
Lñ \\
0
\end{tabular} & $\stackrel{n}{0}$ & & -1 & $\stackrel{1}{0}$ & $\tilde{0}$ & & (ூ) & ?ִ. & $\tilde{0}$ \\
\hline & 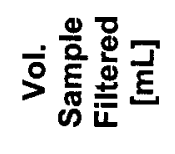 & $\stackrel{\llcorner}{\sim}$ & & $\stackrel{\sim}{N}$ & $\stackrel{\llcorner}{N}$ & $\stackrel{\mathscr{n}}{\sim}$ & & $\stackrel{\stackrel{\sim}{N}}{\sim}$ & $\stackrel{\mathscr{N}}{\sim}$ & $\stackrel{\mathscr{N}}{\sim}$ & & $\stackrel{\Perp}{N}$ & $\stackrel{2}{N}$ & $\stackrel{\mathscr{N}}{ }$ & & $\stackrel{n}{N}$ & $\stackrel{\sim}{\sim}$ & $\stackrel{\Perp}{N}$ & & $\stackrel{\Perp}{\sim}$ & $\stackrel{\sim}{\sim}$ & 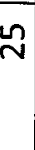 \\
\hline & 施 & 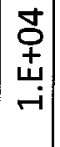 & & 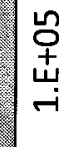 & 㟔 & 告 & & 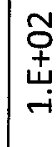 & ن & 足 & & 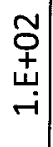 & 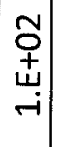 & 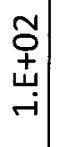 & & 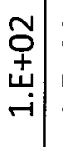 & 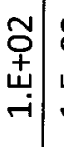 & 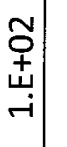 & & 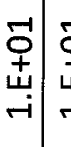 & $\begin{array}{l}-1 \\
\\
+ \\
+ \\
\end{array}$ & . \\
\hline & 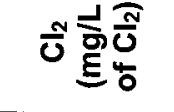 & & & $\frac{8}{\circ}$ & & & & 읃 & & & & $\begin{array}{l}\stackrel{8}{0} \\
\sigma\end{array}$ & & & & $\underset{\infty}{R}$ & & & & ب & & \\
\hline & $\stackrel{\mathscr{E}}{\underline{\underline{E}} . \overline{\underline{\underline{E}}}}$ & & & 0 & & & & in & & & & 늑 & & & & 유 & & & & $\stackrel{\leftrightarrow}{\&}$ & & \\
\hline
\end{tabular}


Table A 32: Chlorine measurements with time for initial chlorine Conc of $0.95 \mathrm{mg} / \mathrm{L} \mathrm{Cl}$

\begin{tabular}{|r|l|}
\hline $\begin{array}{l}\text { Contact Time } \\
(\mathrm{min})\end{array}$ & $\begin{array}{l}\text { Amount of Chlorine } \\
(\mathrm{mg} / \mathrm{L} \mathrm{Cl})\end{array}$ \\
\hline 0 & 0.94 \\
\hline 1 & 0.92 \\
\hline 5 & 0.83 \\
\hline 10 & 0.78 \\
\hline 15 & 0.70 \\
\hline
\end{tabular}

Table A 33: Chlorine measurements with time for initial chlorine Conc of $0.56 \mathrm{mg} / \mathrm{L} \mathrm{Cl}_{2}$

\begin{tabular}{|r|r|}
\hline $\begin{array}{l}\text { Contact Time } \\
(\mathrm{min})\end{array}$ & $\begin{array}{l}\text { Amount of Chlorine } \\
(\mathrm{mg} / \mathrm{L} \mathrm{Cl} 2)\end{array}$ \\
\hline 0 & 0.56 \\
\hline 1 & 0.56 \\
\hline 5 & 0.50 \\
\hline 10 & 0.52 \\
\hline 15 & 0.50 \\
\hline
\end{tabular}

Table A 34: Chlorine measurements with time for initial chlorine Conc of $0.38 \mathrm{mg} / \mathrm{L} \mathrm{Cl}$

\begin{tabular}{|r|l|}
\hline $\begin{array}{l}\text { Contact Time } \\
(\mathrm{min})\end{array}$ & $\begin{array}{l}\text { Amount of Chlorine } \\
(\mathrm{mg} / \mathrm{L} \mathrm{Cl} 2)\end{array}$ \\
\hline 0 & 0.38 \\
\hline 1 & 0.37 \\
\hline 5 & 0.36 \\
\hline 10 & 0.35 \\
\hline 15 & 0.31 \\
\hline
\end{tabular}




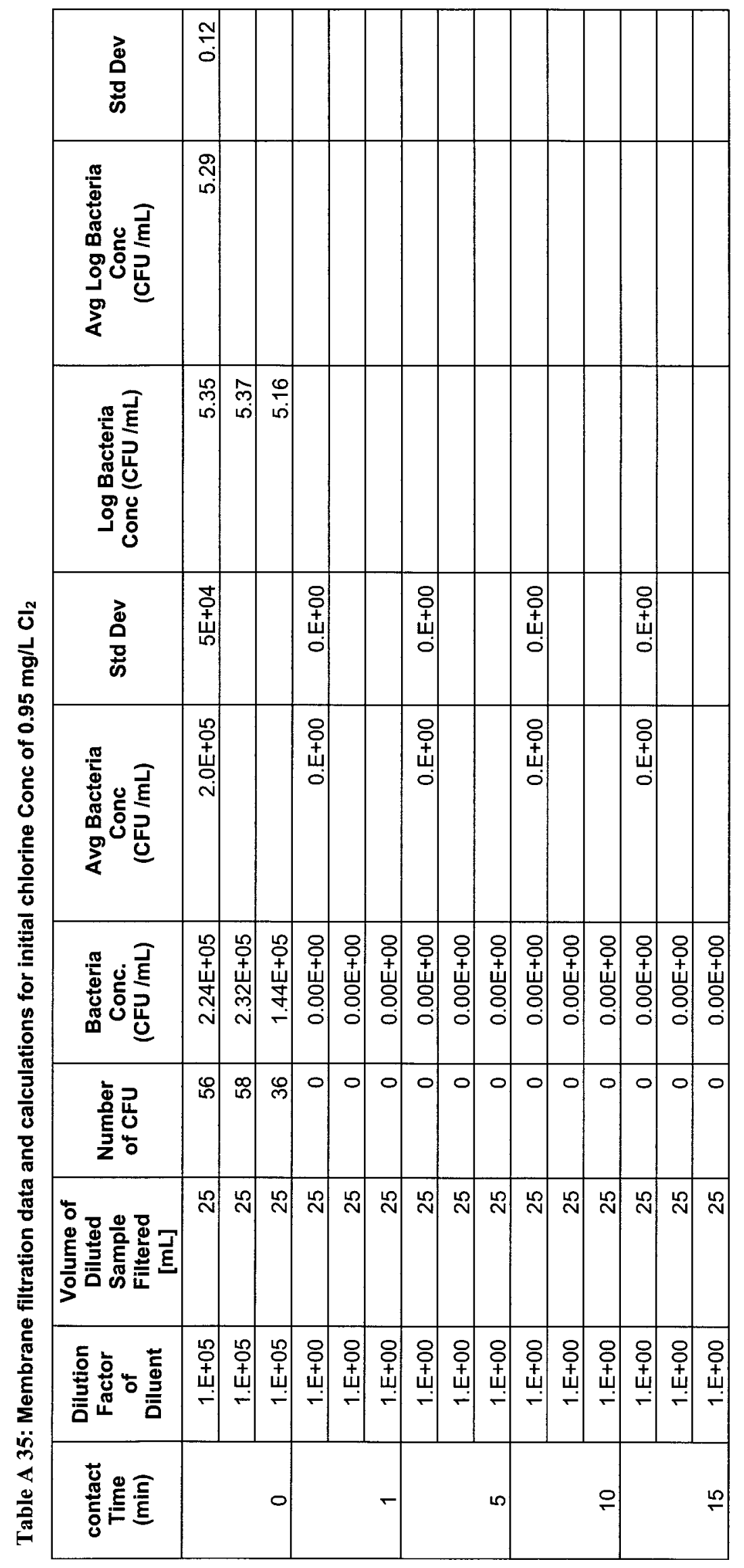


족

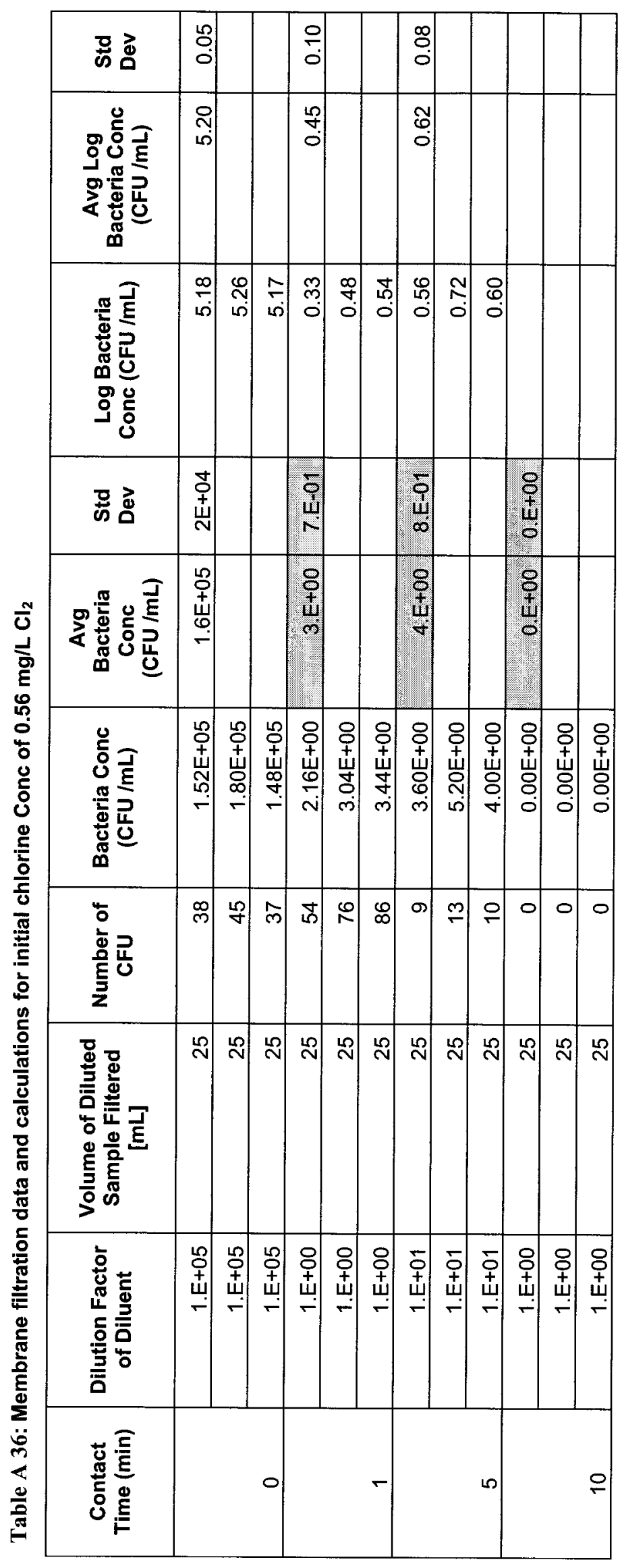




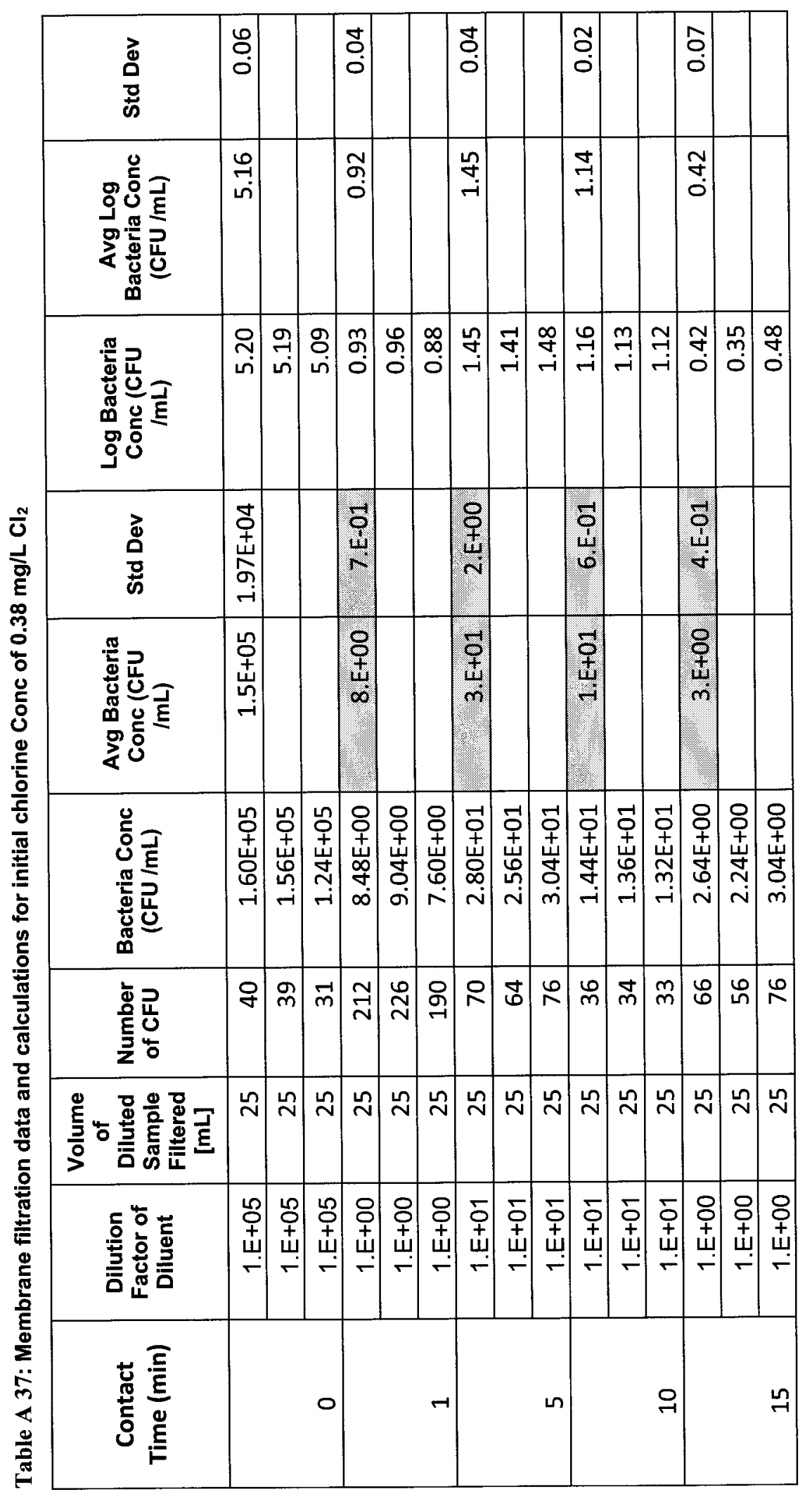


임

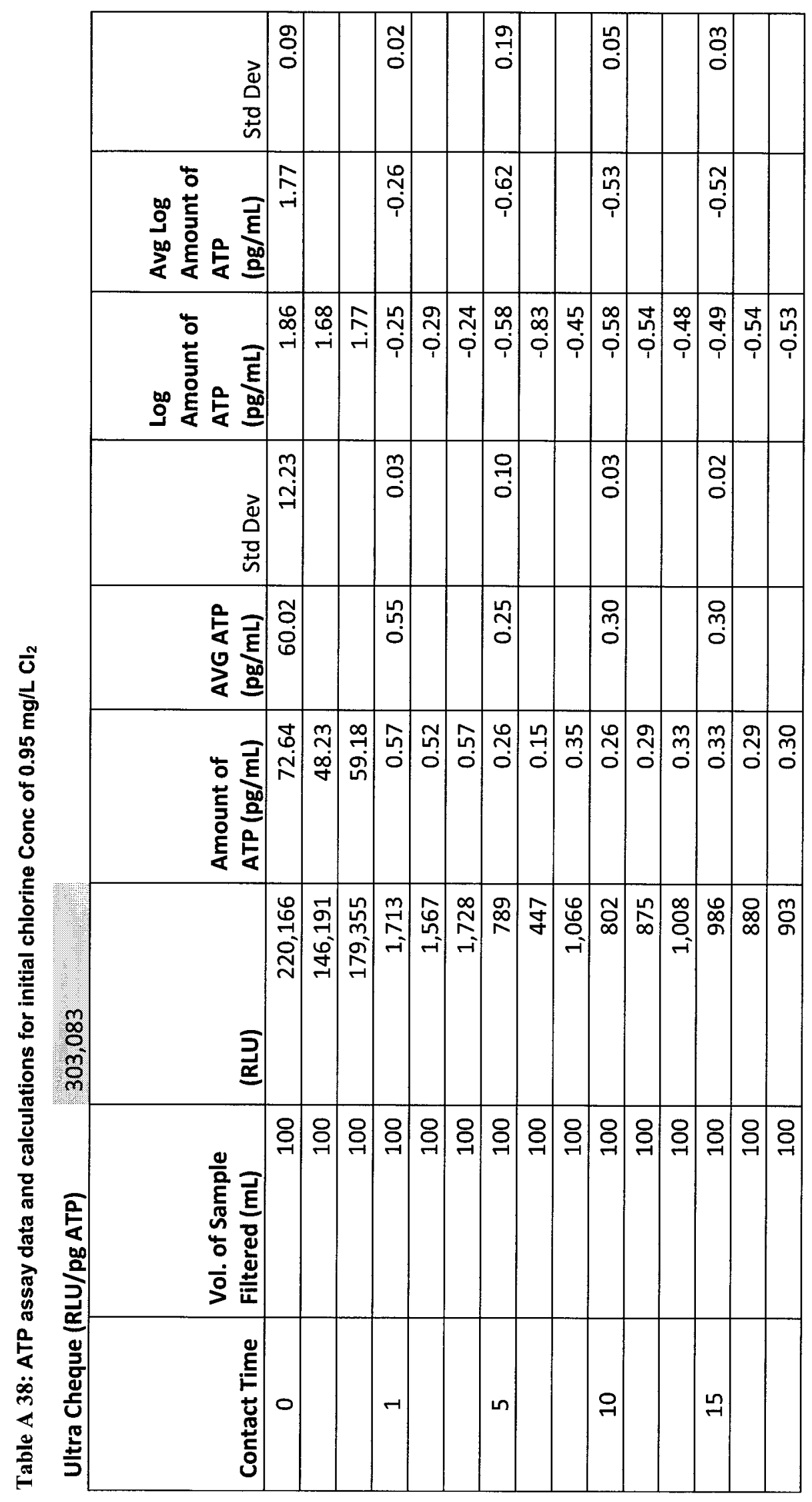


¿

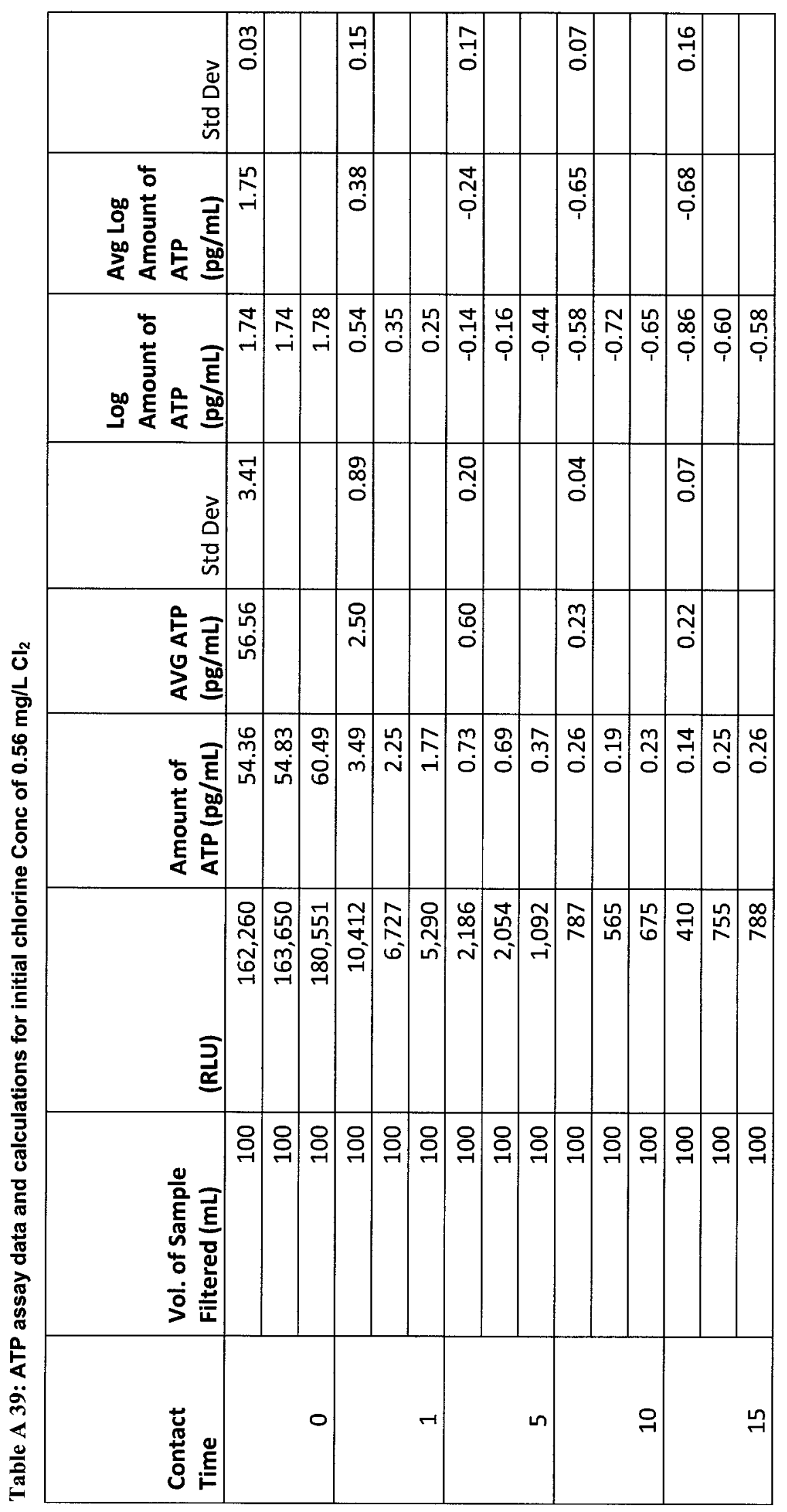


ఫ্ণ

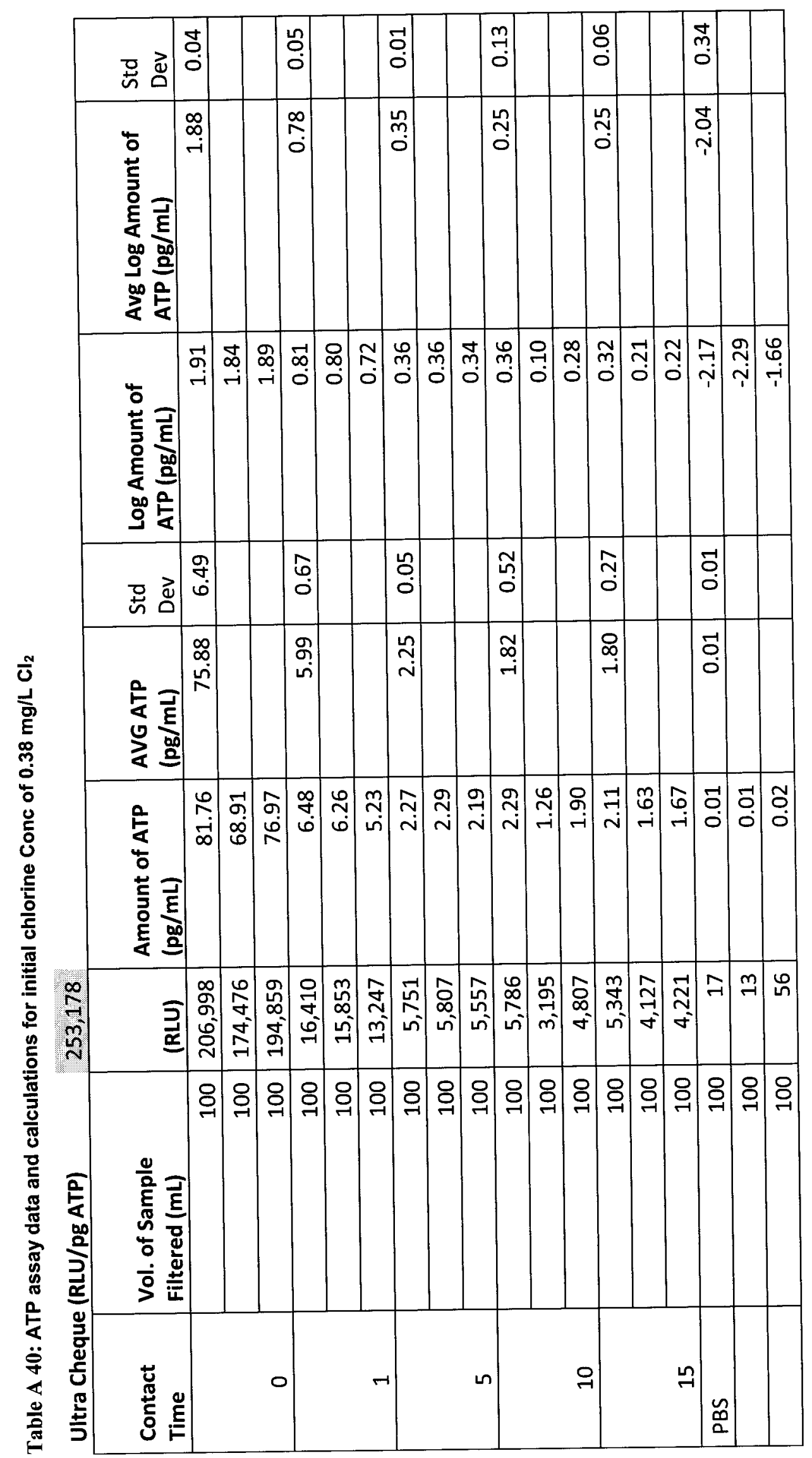

